

\section{DISCLAIMER}

This report was prepared as an account of work sponsored by an agency of the United States Government. Neither the United States Government nor any agency thereof, nor any of their employees, make any warranty, express or implied, or assumes any legal liability or responsibility for the accuracy, completeness, or usefulness of any information, apparatus, product, or process disclosed, or represents that its use would not infringe privately owned rights. Reference herein to any specific commercial product, process, or service by trade name, trademark, manufacturer, or otherwise does not necessarily constitute or imply its endorsement, recommendation, or favoring by the United States Government or any agency thereof. The views and opinions of authors expressed herein do not necessarily state or reflect those of the United States Government or any agency thereof. 


\section{DISCLAIMER}

Portions of this document may be illegible in electronic image products. Images are produced from the best available original document. 


\section{VOLUME 5 SUMMARY}

In 1994, Oak Ridge National Laboratory (ORNL) was tasked by the National Park Service (NPS) to prepare an Environmental Report (ER) for Section 8B of the Foothills Parkway in the Great Smoky Mountains National Park (GSMNP). Section 8B represents 27.7 km (14.2 miles) of a total of $115 \mathrm{~km}$ ( 72 miles) of the planned Foothills Parkway and would connect the Cosby community on the east to the incorporated town of Pittman Center to the west. The major deliverables for the project are listed below.

\begin{tabular}{lc}
\hline Study Plan & August 1994 \\
First Field/Progress Report & October 1994 \\
Second Progress Report & February 1995 \\
Third Progress Report & June 1995 \\
Draft Environmental Report & April 1997 \\
Final Environmental Report & July 1999 \\
\hline
\end{tabular}

From August 1995 through October 1996, NPS, GSMNP, and ORNL staff interacted with Federal Highway Administration staff to develop a conceptual design plan for Section 8B with the intent of protecting critical resources identified during the ER process to the extent possible. In addition, ORNL arranged for bioengineering experts to discuss techniques that might be employed on Section 8B with NPS, GSMNP, and ORNL staff during September 1996.

For the purposes of this ER, there are two basic alternatives under consideration: (1) a build alternative and (2) a no-build alternative. Within the build alternative are a number of options including constructing Section $8 \mathrm{~B}$ with no interchanges, constructing Section $8 \mathrm{~B}$ with an interchange at SR 416 or U.S. 321, constructing Section $8 B$ with a spur road on Webb Mountain, and considering operation of Section $8 \mathrm{~B}$ both before and after the operation of Section $8 \mathrm{C}$. The no-build alternative is considered the no-action alternative and is not to construct Section $8 \mathrm{~B}$.

This volume of the ER consists of Appendices $J$ through $\mathbf{M}$, which describe potential impacts regarding traffic, noise, and aesthetics. The results of the traffic studies described in these appendices resulted in the following conclusions: Unacceptable levels of service will occur on numerous roads within and outside of the park increasingly in the future based upon current and future regional growth. However, the results of the traffic assessment indicated that there would be no significant or cumulative impact from the addition of Section $8 \mathrm{~B}$ or the completion of the Foothills Parkway as a whole within the study area by 2026 . The results of the noise studies described in these appendices resulted in the following four-step plan regarding noise impacts of construction: 
1. Community relations-Early communication with the public is vital. The public should be informed of any potential construction noise impacts as well as procedures planned to mitigate them. Also, a responsive complaint mechanism should be established and publicized for the duration of the project.

2. Design considerations-To the extent possible, construction operations should be located and sequenced to minimize noise impacts near sensitive receptors. Permanent noise barriers planned for the site can be erected early in the construction process to minimize noise, and quieter construction alternatives (e.g., rubber-tired equipment rather than tracked equipment; cast-in piles rather than driven piles) should be used where feasible.

3. Source control-Using newer, quieter equipment or equipment with mufflers will often lessen noise impacts.

4. Site control-Modifying the time, place, or method of operation for particular noise sources can reduce noise impacts. This usually entails limiting the hours of operation near sensitive receptors.

Several recommendations regarding potential impacts to aesthetic resources were also suggested:

- The western exit ramp across the Little Pigeon River should be used.

- The tunnel option east of SR 416 should be selected.

- The SR 416 option rather than the U.S. 321 option should be chosen.

- A ramp should be used at Webb Creek Road if the U.S. 321 option is chosen.

- Both the Webb Mountain lower parking lot and the Webb Mountain loop access road should be built. 


\title{
Appendix J
}

\section{ROADWAY TRAFFIC VOLUME AND LEVEL-OF-SERVICE RESULTS FOR THE FIVE BUILD OPTIONS}

\author{
S. M. Chin
}

Oak Ridge National Laboratory

Oak Ridge, Tennessee

R. Gibson

Oak Ridge National Laboratory

Oak Ridge, Tennessee

August 1995 


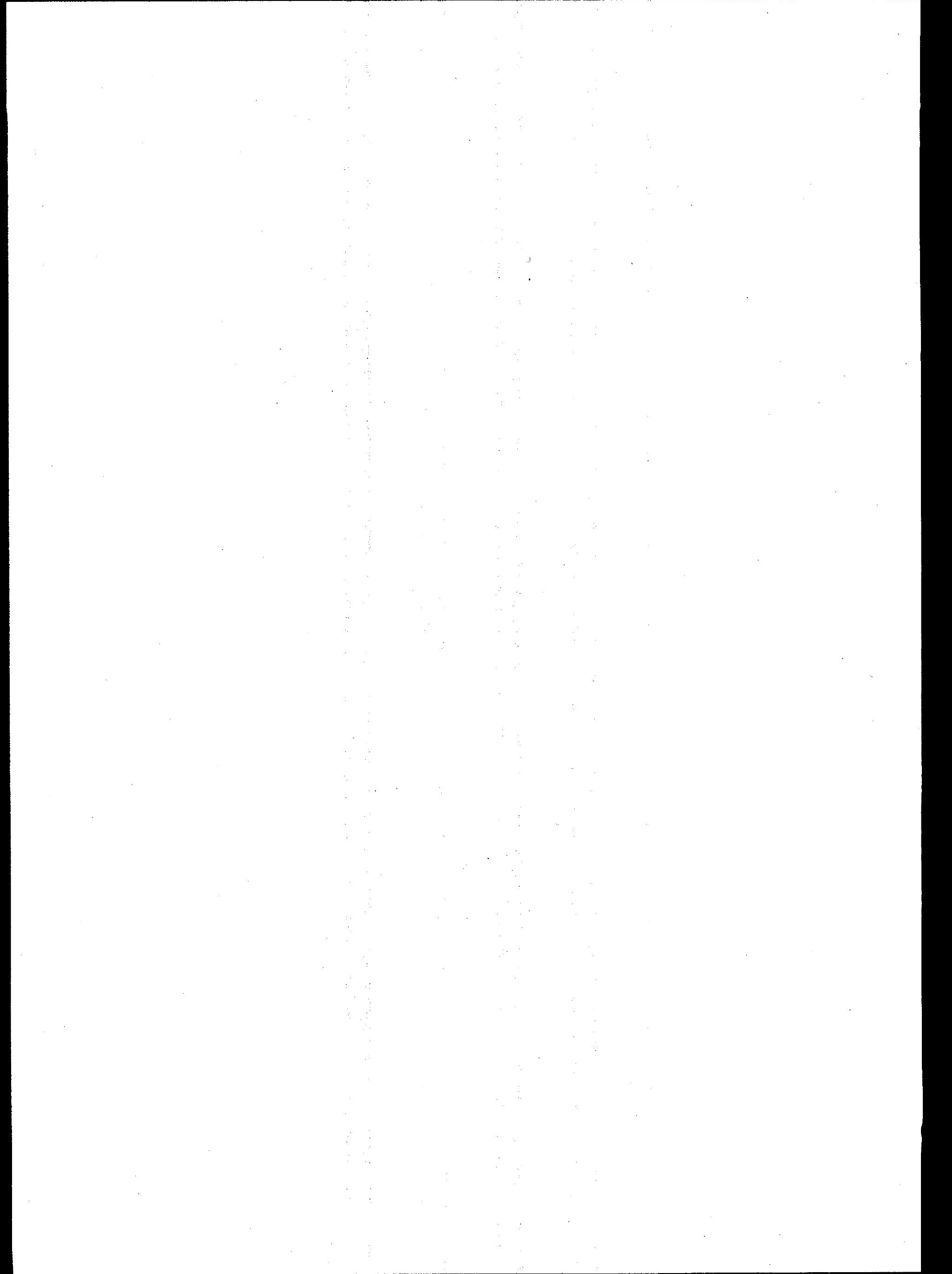




\section{INTRODUCTION}

The traffic projection tables in this appendix list the results of the capacity analyses conducted as part of the traffic study. The traffic conditions predicted by the capacity analyses are discussed in terms of level of service (LOS). For persons not familiar with this terminology, the following discussion is provided in the National Research Council's Highway Capacity Manual of 1994.

\section{Discussion of Level of Service}

The concept of levels of service uses qualitative measures that characterize operational conditions within a traffic stream and their perception by motorists and passengers. The descriptions of individual levels of service characterize these conditions in terms of such factors as speed and travel time, delay, freedom to maneuver, traffic interruptions, and comfort and convenience.

Six levels of service are defined for each type of facility for which analysis procedures are available. They are given letter designations, from A to F, with LOS A representing the best operating conditions and LOS F the worst. Each LOS represents a range of traffic conditions. LOS A represents the highest quality of traffic service, with subsequent LOS categories representing incremental declines in such attributes as travel speed and maneuverability. LOS E corresponds to the maximum flow rate, or capacity, on the facility, while LOS F represents conditions where demand exceeds capacity.

Although higher LOS conditions are more desirable, there is usually a trade-off between construction cost and LOS of service when designing highways. For most design or planning purposes, LOS C and D are typically used. However, acceptable and desirable LOS for highways is usually a decision made by political entities. In this study, we assume LOS A through C to be acceptable for GSMNP and Foothills Parkway roads. For roads outside the park, LOS A through $\mathrm{D}$ is considered acceptable.

Different highway facility types have differing operational goals and characteristics, and travelers have different expectations regarding traffic movement on them. Thus, the procedures for determining LOS for a highway facility, along with the qualitative characteristics of LOS, depend upon the type of facility being analyzed. Most of the roadways within the survey are currently rural two-lane highways, and some will soon be upgraded to rural multilane roads. Therefore, the capacity of each roadway, both for existing and future highway sections, is determined using the procedure appropriate for that facility type. All intersections in the study area are stop-sign controlled, and the corresponding capacity analysis procedures and LOS have been applied. The following paragraphs describe traffic conditions under the six LOS categories for the two types of highways analyzed in this study.

\section{Level of Service for Rural Two-Lane Highways}

LOS A. The highest quality of traffic service. Motorists are able to drive at their desired speed. Without strict enforcement, this can result in speeds approaching the maximum design speed and exceeding posted speed limits (which are usually lower). The passing frequency required to maintain desired speeds has not reached a demanding level, and almost no platoons of three or more vehicles are observed (platoons are vehicles driving together on a highway section, either voluntarily or involuntarily due to signal control, geometrics, or other factors). Drivers would be 
delayed (i.e., would not be able to travel at their desired speed) no more than 30 percent of the time by slow-moving vehicles.

LOS B. Passing demand needed to maintain desired speeds becomes significant and approximately equals the passing capacity at the lower boundary of LOS B. Drivers are delayed up to 45 percent of the time.

LOS C. Noticeable increases in platoon formation, platoon size, and frequency of passing impediments become noticeable. While traffic flow is stable, it is becoming susceptible to congestion due to tuming and slow-moving traffic. Percent time delays can reach 60 percent.

LOS D. Passing becomes extremely difficult as passing demand becomes very high and passing capacity nears zero. Mean platoon sizes of 5 to 10 vehicles are common, and the percentage of time motorists are delayed reaches up to 75 percent.

LOS E. Percent delay time exceeds 75 percent. Passing is virtually impossible under LOS E, and platooning becomes intense when slower vehicles or other interruptions are encountered.

LOS F. This represents heavily congested flow with traffic demand exceeding capacity.

\section{Level of Service for Rural Multilane Highways}

LOS A. Traffic operates under free-flow conditions. Vehicle operation is virtually unaffected by the presence of other vehicles and is only affected by highway geometry and driver preferences. Maneuverability is good, and minor disruptions to flow are easily absorbed without a change in travel speed.

LOS B. This LOS is also indicative of free flow, although the presence of other vehicles begins to be noticeable. Average travel speeds are the same as for LOS A, but drivers have slightly less freedom to maneuver.

LOS C. The influence of traffic density becomes marked. The ability to maneuver within the traffic stream is now clearly affected by the presence of other vehicles, and average travel speeds begin to show some reduction for multilane highways with free-flow speeds over $50 \mathrm{mph}$. Minor disruptions may be expected to cause serious local deterioration in service, and queues may form behind any significant traffic disruption. (A queue is a line of vehicles that is moving very slowly or has stopped, typically at traffic signs or signals or due to some interruption in traffic flow.)

LOS D. The ability to maneuver is severely restricted because of traffic congestion, and travel speed begins to be reduced by increasing volumes. For the majority of multilane highways with free-flow speeds between 45 and $60 \mathrm{mph}$, passenger car speeds at capacity generally range from 44 to $57 \mathrm{mph}$. Only minor disruptions can be absorbed without the formation of extensive queues and the deterioration to LOS E and F.

LOS E. This LOS represents near-capacity conditions and is quite unstable. Vehicles are operating with the minimum spacing at which uniform flow can be maintained. For the majority of multilane highways with free-flow speeds between 45 and $60 \mathrm{mph}$, passenger car speeds at capacity generally range from 42 to $55 \mathrm{mph}$ but are highly variable and unpredictable within that range. As capacity is reached, disruptions cannot be damped or readily dissipated, and most disruptions will cause queues to form and service to deteriorate to LOS F. 
LOS F. This represents forced or breakdown flow. Operations within queues are highly unstable, with vehicles experiencing brief periods of movement followed by stoppages. Average travel times with queues are generally less than $30 \mathrm{mph}$.

\section{Level of Service for Unsignalized Intersections}

Levels of service for movements at unsignalized intersections are determined by the average total delay experienced by vehicles making that movement at the intersection. Total delay, measured in seconds per vehicle, is defined as the total elapsed time from when a vehicle first stops at the end of a queue until the vehicle departs from the stop line. The delay ranges corresponding to each LOS are provided in Table J.1. Note that LOS is not applicable to movements that have a continuous right of way since these vehicles are not required to stop at an intersection.

Table J.1. Level of service criteria for unsignalized intersections

\begin{tabular}{cc}
\hline Level of service & $\begin{array}{c}\text { Average total delay } \\
\text { (seconds per vehicle) }\end{array}$ \\
\hline A & $\leq 5$ \\
B & $>5$ and $\leq 10$ \\
C & $>10$ and $\leq 20$ \\
D & $>20$ and $\leq 30$ \\
E & $>30$ and $\leq 45$ \\
F & $>45$ \\
\hline
\end{tabular}


Table J.2. Weekday A.M. peak hour traffic projections for 2006-construction of Section $8 B$ with no interchanges option

\begin{tabular}{|c|c|c|c|c|c|}
\hline $\begin{array}{c}\text { Road section } \\
\text { name }\end{array}$ & Range & $\begin{array}{c}\text { Traffic } \\
\text { volume }\end{array}$ & Dir & plit & $\begin{array}{l}\text { Percentage } \\
\text { of trucks }\end{array}$ \\
\hline U.S. 321 & $\begin{array}{l}\text { From Intersection with Foothills } \\
\text { Parkway Section } 8 \text { A to } \\
\text { Convergence with SR } 32\end{array}$ & $\begin{array}{r}951 \\
\mathrm{LOS}=\mathrm{B}\end{array}$ & SB & $\begin{array}{l}57 \% \\
43 \%\end{array}$ & $9.00 \%$ \\
\hline U.S. 321 & $\begin{array}{l}\text { From U.S. } 321 \text { Convergence } \\
\text { with SR } 32 \text { to Intersection with } \\
\text { SR } 416\end{array}$ & $\begin{array}{r}805 \\
L O S=D\end{array}$ & WB & $\begin{array}{l}53 \% \\
47 \%\end{array}$ & $7.00 \%$ \\
\hline SR 416 & $\begin{array}{l}\text { From Intersection with U.S. } 321 \\
\text { to Intersection with Webb Creek } \\
\mathrm{Rd}\end{array}$ & $\begin{array}{r}214 \\
\mathrm{LOS}=\mathrm{B}\end{array}$ & SB & $\begin{array}{l}49 \% \\
51 \%\end{array}$ & $7.00 \%$ \\
\hline U.S. 321 & $\begin{array}{l}\text { From Intersection with SR } 416 \\
\text { to Outside of Gatlinburg }\end{array}$ & $\begin{array}{r}1151 \\
\text { LOS }=B\end{array}$ & $\begin{array}{l}\text { EB } \\
\text { WB }\end{array}$ & $\begin{array}{l}36 \% \\
64 \%\end{array}$ & $5.00 \%$ \\
\hline Foothill 8B & From Cosby to Pittman Center & $\begin{array}{r}186 \\
L O S=B \\
\end{array}$ & $\begin{array}{l}\text { EB } \\
\text { WB }\end{array}$ & $\begin{array}{l}52 \% \\
48 \% \\
\end{array}$ & $0.00 \%$ \\
\hline
\end{tabular}

Table J.3. Weekday A.M. peak hour traffic projections for 2026-construction of Section $8 \mathrm{~B}$ with no interchanges option

\begin{tabular}{|c|c|c|c|c|c|}
\hline $\begin{array}{c}\text { Road section } \\
\text { name }\end{array}$ & Range & $\begin{array}{l}\text { Traffic } \\
\text { volume }\end{array}$ & \multicolumn{2}{|c|}{ Directional split } & $\begin{array}{c}\text { Percentage } \\
\text { of trucks }\end{array}$ \\
\hline U.S. 321 & $\begin{array}{l}\text { From Intersection with Foothills } \\
\text { Parkway Section 8A to } \\
\text { Convergence with SR } 32\end{array}$ & $\begin{array}{r}1242 \\
\mathrm{LOS}=\mathrm{B}\end{array}$ & NB & $\begin{array}{l}56 \% \\
44 \%\end{array}$ & $9.00 \%$ \\
\hline U.S. 321 & $\begin{array}{l}\text { From U.S. } 321 \text { Convergence } \\
\text { with SR } 32 \text { to Intersection with } \\
\text { SR } 416\end{array}$ & $\begin{array}{r}1118 \\
\mathrm{LOS}=\mathrm{D}\end{array}$ & WB & $\begin{array}{l}55 \% \\
45 \%\end{array}$ & $7.00 \%$ \\
\hline SR 416 & $\begin{array}{l}\text { From Intersection with U.S. } 321 \\
\text { to Intersection with Webb Creek } \\
\text { Rd }\end{array}$ & $\begin{array}{r}247 \\
\mathrm{LOS}=\mathrm{B}\end{array}$ & $\begin{array}{l}\text { NB } \\
\text { SB }\end{array}$ & $\begin{array}{l}36 \% \\
64 \%\end{array}$ & $7.00 \%$ \\
\hline U.S. 321 & $\begin{array}{l}\text { From Intersection with SR } 416 \\
\text { to Outside of Gatlinburg }\end{array}$ & $\begin{array}{r}1571 \\
\operatorname{LOS}=\mathrm{C}\end{array}$ & WB & $\begin{array}{l}30 \% \\
70 \%\end{array}$ & $5.00 \%$ \\
\hline Foothill 8B & From Cosby to Pittman Center & $\begin{array}{r}263 \\
\operatorname{LOS}=C\end{array}$ & $\begin{array}{l}\text { EB } \\
\text { WB }\end{array}$ & $\begin{array}{l}58 \% \\
42 \%\end{array}$ & $0.00 \%$ \\
\hline
\end{tabular}


Table J.4. Weekday P.M. peak hour traffic projections for 2006-construction of Section 8B with no interchanges option

\begin{tabular}{|c|c|c|c|c|c|}
\hline $\begin{array}{l}\text { Road section } \\
\text { name }\end{array}$ & Range & $\begin{array}{l}\text { Traffic } \\
\text { volume } \\
\end{array}$ & Dir & plit & $\begin{array}{c}\text { Percentage } \\
\text { of trucks }\end{array}$ \\
\hline U.S. 321 & $\begin{array}{l}\text { From Intersection with Foothills } \\
\text { Parkway Section } 8 \text { A to } \\
\text { Convergence with SR } 32\end{array}$ & $\begin{array}{r}1005 \\
\mathrm{LOS}=\mathrm{B}\end{array}$ & NB & $\begin{array}{l}59 \% \\
41 \%\end{array}$ & $9.00 \%$ \\
\hline U.S. 321 & $\begin{array}{l}\text { From U.S. } 321 \text { Convergence } \\
\text { with SR } 32 \text { to Intersection with } \\
\text { SR } 416\end{array}$ & $\begin{array}{r}846 \\
\mathrm{LOS}=\mathrm{D}\end{array}$ & WB & $\begin{array}{l}51 \% \\
49 \%\end{array}$ & $7.00 \%$ \\
\hline SR 416 & $\begin{array}{l}\text { From Intersection with U.S. } 321 \\
\text { to Intersection with Webb Creek } \\
\text { Rd }\end{array}$ & $\begin{array}{r}203 \\
\mathrm{LOS}=\mathrm{B}\end{array}$ & NB & $\begin{array}{l}54 \% \\
46 \%\end{array}$ & $7.00 \%$ \\
\hline U.S. 321 & $\begin{array}{l}\text { From Intersection with SR } 416 \\
\text { to Outside of Gatlinburg }\end{array}$ & $\begin{array}{r}933 \\
\text { LOS }=B\end{array}$ & WB & $\begin{array}{l}28 \% \\
72 \%\end{array}$ & $5.00 \%$ \\
\hline Foothill 8B & From Cosby to Pittman Center & $\begin{array}{r}221 \\
\mathrm{LOS}=\mathrm{C}\end{array}$ & $\begin{array}{l}\text { EB } \\
\text { WB }\end{array}$ & $\begin{array}{l}58 \% \\
42 \% \\
\end{array}$ & $0.00 \%$ \\
\hline
\end{tabular}

Table J.5. Weekday P.M. peak hour traffic projections for 2026-construction of Section $8 B$ with no interchanges option

\begin{tabular}{llrrrr}
\hline $\begin{array}{c}\text { Road section } \\
\text { name }\end{array}$ & \multicolumn{1}{c}{ Range } & $\begin{array}{c}\text { Traffic } \\
\text { volume }\end{array}$ & \multicolumn{1}{c}{ Directional split } & $\begin{array}{c}\text { Percentage } \\
\text { of trucks }\end{array}$ \\
\hline U.S. 321 & $\begin{array}{l}\text { From Intersection with Foothills } \\
\text { Parkway Section 8A to } \\
\text { Convergence with SR 32 }\end{array}$ & 1316 & NB & $59 \%$ & $9.00 \%$ \\
& $\begin{array}{l}\text { From U.S. 321 Convergence } \\
\text { U.S. 321 }\end{array}$ & 1152 & WB & $52 \%$ & $7.00 \%$ \\
& $\begin{array}{l}\text { SR 416 } \\
\text { SR 32 to Intersection with }\end{array}$ & LOS=D & EB & $48 \%$ & \\
SR 416 & $\begin{array}{l}\text { From Intersection with U.S. 321 } \\
\text { to Intersection with Webb Creek }\end{array}$ & 245 & NB & $43 \%$ & $7.00 \%$ \\
Rd & LOS=B & SB & $57 \%$ & \\
U.S. 321 & $\begin{array}{l}\text { From Intersection with SR 416 } \\
\text { to Outside of Gatlinburg }\end{array}$ & 1395 & EB & $28 \%$ & $5.00 \%$ \\
Foothill 8B & From Cosby to Pittman Center & 313 & EB & $64 \%$ & $0.00 \%$ \\
& & LOS=B & WB & $72 \%$ & \\
\hline
\end{tabular}


Table J.6. Weekend A.M. peak hour traffic projections for 2006-construction of Section $8 B$ with no interchanges option

\begin{tabular}{llrrrr}
\hline $\begin{array}{c}\text { Road section } \\
\text { name }\end{array}$ & \multicolumn{1}{c}{ Range } & $\begin{array}{c}\text { Traffic } \\
\text { volume }\end{array}$ & Directional split & $\begin{array}{c}\text { Percentage } \\
\text { of trucks }\end{array}$ \\
\hline U.S. 321 & $\begin{array}{l}\text { From Intersection with Foothills } \\
\text { Parkway Section 8A to } \\
\text { Convergence with SR 32 }\end{array}$ & 1333 & NB & $52 \%$ & $9.00 \%$ \\
U.S. 321 & $\begin{array}{l}\text { From U.S. 321 Convergence } \\
\text { with SR 32 to Intersection with }\end{array}$ & 1104 & WB & $45 \%$ & $7.00 \%$ \\
& SR 416 & LOS=D & EB & $55 \%$ & \\
SR 416 & $\begin{array}{l}\text { From Intersection with U.S. 321 } \\
\text { to Intersection with Webb Creek }\end{array}$ & 219 & NB & $54 \%$ & $7.00 \%$ \\
U.S. 321 & $\begin{array}{l}\text { Rd } \\
\text { From Intersection with SR 416 }\end{array}$ & 1413 & EB & $38 \%$ & $5.00 \%$ \\
to Outside of Gatlinburg & LOS=B & WB & $62 \%$ & \\
& & 241 & EB & $55 \%$ & $0.00 \%$ \\
\hline
\end{tabular}

Table J.7. Weekend A.M. peak hour traffic projections for 2026-construction of Section $8 \mathrm{~B}$ with no interchanges option

\begin{tabular}{|c|c|c|c|c|c|}
\hline $\begin{array}{c}\text { Road section } \\
\text { name }\end{array}$ & Range & $\begin{array}{l}\text { Traffic } \\
\text { volume }\end{array}$ & \multicolumn{2}{|c|}{ Directional split } & $\begin{array}{l}\text { Percentage } \\
\text { of trucks }\end{array}$ \\
\hline U.S. 321 & $\begin{array}{l}\text { From Intersection with Foothills } \\
\text { Parkway Section } 8 \text { A to } \\
\text { Convergence with SR } 32\end{array}$ & $\begin{array}{r}1746 \\
\mathrm{LOS}=\mathrm{C}\end{array}$ & $\begin{array}{l}\text { NB } \\
\text { SB }\end{array}$ & $\begin{array}{l}51 \% \\
49 \%\end{array}$ & $9.00 \%$ \\
\hline U.S. 321 & $\begin{array}{l}\text { From U.S. } 321 \text { Convergence } \\
\text { with SR } 32 \text { to Intersection with } \\
\text { SR } 416\end{array}$ & $\begin{array}{r}1530 \\
\mathrm{LOS}=\mathrm{E}\end{array}$ & $\begin{array}{l}\text { WB } \\
\text { EB }\end{array}$ & $\begin{array}{l}57 \% \\
43 \%\end{array}$ & $7.00 \%$ \\
\hline SR 416 & $\begin{array}{l}\text { From Intersection with U.S. } 321 \\
\text { to Intersection with Webb Creek } \\
\text { Rd }\end{array}$ & $\begin{array}{r}231 \\
\mathrm{LOS}=\mathrm{B}\end{array}$ & $\begin{array}{l}\text { NB } \\
\text { SB }\end{array}$ & $\begin{array}{l}38 \% \\
62 \%\end{array}$ & $7.00 \%$ \\
\hline U.S. 321 & $\begin{array}{l}\text { From Intersection with SR } 416 \\
\text { to Outside of Gatlinburg }\end{array}$ & $\begin{array}{r}1916 \\
\text { LOS }=C\end{array}$ & $\mathrm{~EB}$ & $\begin{array}{l}31 \% \\
69 \%\end{array}$ & $5.00 \%$ \\
\hline Foothill 8B & From Cosby to Pittman Center & $\begin{array}{r}348 \\
\text { LOS }=C\end{array}$ & $\begin{array}{l}\text { EB } \\
\text { WB }\end{array}$ & $\begin{array}{l}60 \% \\
40 \% \\
\end{array}$ & $0.00 \%$ \\
\hline
\end{tabular}


Table J.8. Weekend P.M. peak hour traffic projections for 2006-construction of Section 8B with no interchanges option

\begin{tabular}{|c|c|c|c|c|c|}
\hline $\begin{array}{c}\text { Road section } \\
\text { name }\end{array}$ & Range & $\begin{array}{c}\text { Traffic } \\
\text { volume }\end{array}$ & \multicolumn{2}{|c|}{ Directional split } & $\begin{array}{c}\text { Percentage } \\
\text { of trucks }\end{array}$ \\
\hline U.S. 321 & $\begin{array}{l}\text { From Intersection with Foothills } \\
\text { Parkway Section } 8 \text { A to } \\
\text { Convergence with SR } 32\end{array}$ & $\begin{array}{r}1273 \\
\mathrm{LOS}=\mathrm{B}\end{array}$ & $\begin{array}{l}\text { NB } \\
\text { SB }\end{array}$ & $\begin{array}{l}55 \% \\
45 \%\end{array}$ & $9.00 \%$ \\
\hline U.S. 321 & $\begin{array}{l}\text { From U.S. } 321 \text { Convergence } \\
\text { with SR } 32 \text { to Intersection with } \\
\text { SR } 416\end{array}$ & $\begin{array}{r}1156 \\
\mathrm{LOS}=\mathrm{D}\end{array}$ & $\begin{array}{l}\text { WB } \\
\text { EB }\end{array}$ & $\begin{array}{l}54 \% \\
46 \%\end{array}$ & $7.00 \%$ \\
\hline SR 416 & $\begin{array}{l}\text { From Intersection with U.S. } 321 \\
\text { to Intersection with Webb Creek } \\
\text { Rd }\end{array}$ & $\begin{array}{r}269 \\
\operatorname{LOS}=B\end{array}$ & $\begin{array}{l}\text { NB } \\
\text { SB }\end{array}$ & $\begin{array}{l}49 \% \\
51 \%\end{array}$ & $7.00 \%$ \\
\hline U.S. 321 & $\begin{array}{l}\text { From Intersection with SR } 416 \\
\text { to Outside of Gatlinburg }\end{array}$ & $\begin{array}{r}1304 \\
\text { LOS }=B\end{array}$ & WB & $\begin{array}{l}28 \% \\
72 \%\end{array}$ & $5.00 \%$ \\
\hline Foothill 8B & From Cosby to Pittman Center & $\begin{array}{r}264 \\
\mathrm{LOS}=\mathrm{C}\end{array}$ & $\begin{array}{l}\text { EB } \\
\text { WB }\end{array}$ & $\begin{array}{l}58 \% \\
42 \% \\
\end{array}$ & $0.00 \%$ \\
\hline
\end{tabular}

Table J.9. Weekend P.M. peak hour traffic projections for 2026-construction of Section 8B with no interchanges option

\begin{tabular}{|c|c|c|c|c|c|}
\hline $\begin{array}{c}\text { Road section } \\
\text { name }\end{array}$ & Range & $\begin{array}{c}\text { Traffic } \\
\text { volume } \\
\end{array}$ & Dir & split & $\begin{array}{l}\text { Percentage } \\
\text { of trucks }\end{array}$ \\
\hline U.S. 321 & $\begin{array}{l}\text { From Intersection with Foothills } \\
\text { Parkway Section 8A to } \\
\text { Convergence with SR } 32\end{array}$ & $\begin{array}{r}1671 \\
\mathrm{LOS}=\mathrm{C}\end{array}$ & $\begin{array}{l}\text { NB } \\
\text { SB }\end{array}$ & $\begin{array}{l}54 \% \\
46 \%\end{array}$ & $9.00 \%$ \\
\hline U.S. 321 & $\begin{array}{l}\text { From U.S. } 321 \text { Convergence } \\
\text { with SR } 32 \text { to Intersection with } \\
\text { SR } 416\end{array}$ & $\begin{array}{r}1577 \\
\mathrm{LOS}=\mathrm{E}\end{array}$ & $\begin{array}{l}\text { WB } \\
\text { EB }\end{array}$ & $\begin{array}{l}55 \% \\
45 \%\end{array}$ & $7.00 \%$ \\
\hline SR 416 & $\begin{array}{l}\text { From Intersection with U.S. } 321 \\
\text { to Intersection with Webb Creek } \\
\text { Rd }\end{array}$ & $\begin{array}{r}315 \\
\mathrm{LOS}=\mathrm{B}\end{array}$ & $\begin{array}{l}\text { NB } \\
\text { SB }\end{array}$ & $\begin{array}{l}37 \% \\
63 \%\end{array}$ & $7.00 \%$ \\
\hline U.S. 321 & $\begin{array}{l}\text { From Intersection with SR } 416 \\
\text { to Outside of Gatlinburg }\end{array}$ & $\begin{array}{r}1957 \\
\text { LOS }=\mathrm{C}\end{array}$ & WB & $\begin{array}{l}29 \% \\
71 \%\end{array}$ & $5.00 \%$ \\
\hline Foothill 8B & From Cosby to Pittman Center & $\begin{array}{r}376 \\
L O S=C\end{array}$ & $\begin{array}{l}\text { EB } \\
\text { WB }\end{array}$ & $\begin{array}{l}64 \% \\
36 \%\end{array}$ & $0.00 \%$ \\
\hline
\end{tabular}


Table J.10. Weekday A.M. peak hour traffic projections for 2006-interchange at Highway 416 (both options)-8B not opened until $8 \mathrm{C}$ completed

\begin{tabular}{|c|c|c|c|c|c|}
\hline $\begin{array}{c}\text { Road section } \\
\text { name }\end{array}$ & Range & $\begin{array}{l}\text { Traffic } \\
\text { volume }\end{array}$ & \multicolumn{2}{|c|}{ Directional split } & $\begin{array}{l}\text { Percentage } \\
\text { of trucks }\end{array}$ \\
\hline U.S. 321 & $\begin{array}{l}\text { From Intersection with Foothills } \\
\text { Parkway Section 8A to } \\
\text { Convergence with SR } 32\end{array}$ & $\begin{array}{r}951 \\
\mathrm{LOS}=\mathrm{B}\end{array}$ & SB & $\begin{array}{l}57 \% \\
43 \%\end{array}$ & $9.00 \%$ \\
\hline U.S. 321 & $\begin{array}{l}\text { From U.S. } 321 \text { Convergence } \\
\text { with SR } 32 \text { to Intersection with } \\
\text { SR } 416\end{array}$ & $\begin{array}{r}833 \\
\mathrm{LOS}=\mathrm{D}\end{array}$ & $\begin{array}{l}\text { WB } \\
\text { EB }\end{array}$ & $\begin{array}{l}55 \% \\
45 \%\end{array}$ & $7.00 \%$ \\
\hline SR 416 & $\begin{array}{l}\text { From Intersection with U.S. } 321 \\
\text { to Intersection with Webb Creek } \\
\text { Rd }\end{array}$ & $\begin{array}{r}241 \\
\mathrm{LOS}=\mathrm{B}\end{array}$ & $\begin{array}{l}\text { NB } \\
\text { SB }\end{array}$ & $\begin{array}{l}69 \% \\
31 \%\end{array}$ & $7.00 \%$ \\
\hline U.S. 321 & $\begin{array}{l}\text { From Intersection with SR } 416 \\
\text { to Outside of Gatlinburg }\end{array}$ & $\begin{array}{r}1106 \\
\operatorname{LOS}=B\end{array}$ & $\begin{array}{l}\text { EB } \\
\text { WB }\end{array}$ & $\begin{array}{l}36 \% \\
64 \%\end{array}$ & $5.00 \%$ \\
\hline Foothill 8B & From Cosby to Pittman Center & $\begin{array}{r}186 \\
\text { LOS }=B\end{array}$ & $\begin{array}{l}\text { EB } \\
\text { WB }\end{array}$ & $\begin{array}{l}52 \% \\
48 \%\end{array}$ & $0.00 \%$ \\
\hline
\end{tabular}

Table J.11. Weekday A.M. peak hour traffic projections for 2026-interchange at Highway 416 (both options) $-8 B$ not opened until $8 \mathrm{C}$ completed

\begin{tabular}{|c|c|c|c|c|c|}
\hline $\begin{array}{c}\text { Road section } \\
\text { name }\end{array}$ & Range & $\begin{array}{l}\text { Traffic } \\
\text { volume }\end{array}$ & \multicolumn{2}{|c|}{ Directional split } & $\begin{array}{c}\text { Percentage } \\
\text { of trucks }\end{array}$ \\
\hline U.S. 321 & $\begin{array}{l}\text { From Intersection with Foothills } \\
\text { Parkway Section } 8 \text { A to } \\
\text { Convergence with SR } 32\end{array}$ & $\begin{array}{r}1242 \\
\text { LOS }=B\end{array}$ & SB & $\begin{array}{l}56 \% \\
44 \%\end{array}$ & $9.00 \%$ \\
\hline U.S. 321 & $\begin{array}{l}\text { From U.S. } 321 \text { Convergence } \\
\text { with SR } 32 \text { to Intersection with } \\
\text { SR } 416\end{array}$ & $\begin{array}{r}1116 \\
\text { LOS }=D\end{array}$ & WB & $\begin{array}{l}55 \% \\
45 \%\end{array}$ & $7.00 \%$ \\
\hline SR 416 & $\begin{array}{l}\text { From Intersection with U.S. } 321 \\
\text { to Intersection with Webb Creek } \\
\text { Rd }\end{array}$ & $\begin{array}{r}237 \\
\text { LOS }=B\end{array}$ & SB & $\begin{array}{l}63 \% \\
37 \%\end{array}$ & $7.00 \%$ \\
\hline U.S. 321 & $\begin{array}{l}\text { From Intersection with SR } 416 \\
\text { to Outside of Gatlinburg }\end{array}$ & $\begin{array}{r}1433 \\
\text { LOS }=\mathrm{B}\end{array}$ & WB & $32 \%$ & $5.00 \%$ \\
\hline Foothill 8B & From Cosby to Pittman Center & $\begin{array}{r}263 \\
\mathrm{LOS}=\mathrm{C}\end{array}$ & $\begin{array}{l}\text { EB } \\
\text { WB }\end{array}$ & $\begin{array}{l}58 \% \\
42 \% \\
\end{array}$ & $0.00 \%$ \\
\hline
\end{tabular}


Table J.12. Weekday P.M. peak hour traffic projections for 2006-interchange at Highway 416 (both options) $-8 B$ not opened until $8 \mathrm{C}$ completed

\begin{tabular}{llrrrr}
\hline $\begin{array}{c}\text { Road section } \\
\text { name }\end{array}$ & \multicolumn{1}{c}{ Range } & $\begin{array}{c}\text { Traffic } \\
\text { volume }\end{array}$ & Directional split & $\begin{array}{c}\text { Percentage } \\
\text { of trucks }\end{array}$ \\
\hline U.S. 321 & $\begin{array}{l}\text { From Intersection with Foothills } \\
\text { Parkway Section 8A to } \\
\text { Convergence with SR 32 }\end{array}$ & 1005 & NB & $59 \%$ & $9.00 \%$ \\
U.S. 321 & $\begin{array}{l}\text { From U.S. 321 Convergence } \\
\text { with SR 32 to Intersection with }\end{array}$ & 908 & WB & $54 \%$ & $7.00 \%$ \\
& SR 416 & LOS=D & EB & $46 \%$ & \\
SR 416 & $\begin{array}{l}\text { From Intersection with U.S. 321 } \\
\text { to Intersection with Webb Creek }\end{array}$ & 283 & NB & $78 \%$ & $7.00 \%$ \\
& Rd & LOS=B & SB & $22 \%$ & \\
U.S. 321 & From Intersection with SR 416 & 965 & EB & $33 \%$ & $5.00 \%$ \\
& to Outside of Gatlinburg & LOS=B & WB & $67 \%$ & \\
Foothill 8B & From Cosby to Pittman Center & 221 & EB & $58 \%$ & $0.00 \%$ \\
& & LOS=C & WB & $42 \%$ & \\
\hline
\end{tabular}

Table J.13. Weekday. P.M. peak hour traffic projections for 2026-interchange at Highway 416 (both options)-8B not opened until 8C completed

\begin{tabular}{|c|c|c|c|c|c|}
\hline $\begin{array}{c}\text { Road section } \\
\text { name } \\
\end{array}$ & Range & $\begin{array}{l}\text { Traffic } \\
\text { volume }\end{array}$ & & split & $\begin{array}{l}\text { Percentage } \\
\text { of trucks }\end{array}$ \\
\hline U.S. 321 & $\begin{array}{l}\text { From Intersection with Foothills } \\
\text { Parkway Section } 8 \text { A to } \\
\text { Convergence with SR } 32\end{array}$ & $\begin{array}{r}1316 \\
\text { LOS }=B\end{array}$ & $\begin{array}{l}\text { NB } \\
\text { SB }\end{array}$ & $\begin{array}{l}59 \% \\
41 \%\end{array}$ & $9.00 \%$ \\
\hline U.S. 321 & $\begin{array}{l}\text { From U.S. } 321 \text { Convergence } \\
\text { with SR } 32 \text { to Intersection with } \\
\text { SR } 416\end{array}$ & $\begin{array}{r}1189 \\
\text { LOS }=D\end{array}$ & $\begin{array}{l}\text { WB } \\
\text { EB }\end{array}$ & $\begin{array}{l}53 \% \\
47 \%\end{array}$ & $7.00 \%$ \\
\hline SR 416 & $\begin{array}{l}\text { From Intersection with U.S. } 321 \\
\text { to Intersection with Webb Creek } \\
\text { Rd }\end{array}$ & $\begin{array}{r}289 \\
\mathrm{LOS}=\mathrm{B}\end{array}$ & $\begin{array}{l}\text { NB } \\
\text { SB }\end{array}$ & $\begin{array}{l}76 \% \\
24 \%\end{array}$ & $7.00 \%$ \\
\hline U.S. 321 & $\begin{array}{l}\text { From Intersection with SR } 416 \\
\text { to Outside of Gatlinburg }\end{array}$ & $\begin{array}{r}1286 \\
\mathrm{LOS}=\mathrm{B}\end{array}$ & $\mathrm{EB}$ & $\begin{array}{l}30 \% \\
70 \%\end{array}$ & $5.00 \%$ \\
\hline Foothill 8B & From Cosby to Pittman Center & $\begin{array}{r}313 \\
\operatorname{LOS}=\mathrm{C}\end{array}$ & $\begin{array}{l}\text { EB } \\
\text { WB }\end{array}$ & $\begin{array}{l}64 \% \\
36 \%\end{array}$ & $0.00 \%$ \\
\hline
\end{tabular}


Table J.14. Weekend A.M. peak hour traffic projections for 2006-interchange at Highway 416 (both options) $-8 B$ not opened until $8 \mathrm{C}$ completed

\begin{tabular}{|c|c|c|c|c|c|}
\hline $\begin{array}{c}\text { Road section } \\
\text { name }\end{array}$ & Range & $\begin{array}{l}\text { Traffic } \\
\text { volume }\end{array}$ & \multicolumn{2}{|c|}{ Directional split } & $\begin{array}{l}\text { Percentage } \\
\text { of trucks }\end{array}$ \\
\hline U.S. 321 & $\begin{array}{l}\text { From Intersection with Foothills } \\
\text { Parkway Section } 8 \text { A to } \\
\text { Convergence with SR } 32\end{array}$ & $\begin{array}{r}1333 \\
\mathrm{LOS}=\mathrm{B}\end{array}$ & SB & $\begin{array}{l}52 \% \\
48 \%\end{array}$ & $9.00 \%$ \\
\hline U.S. 321 & $\begin{array}{l}\text { From U.S. } 321 \text { Convergence } \\
\text { with SR } 32 \text { to Intersection with } \\
\text { SR } 416\end{array}$ & $\begin{array}{r}1140 \\
\mathrm{LOS}=\mathrm{D}\end{array}$ & $\begin{array}{l}\text { WB } \\
\mathrm{EB}\end{array}$ & $\begin{array}{l}57 \% \\
43 \%\end{array}$ & $7.00 \%$ \\
\hline SR 416 & $\begin{array}{l}\text { From Intersection with U.S. } 321 \\
\text { to Intersection with Webb Creek } \\
\text { Rd }\end{array}$ & $\begin{array}{r}247 \\
\text { LOS }=B\end{array}$ & SB & $\begin{array}{l}73 \% \\
27 \%\end{array}$ & $7.00 \%$ \\
\hline U.S. 321 & $\begin{array}{l}\text { From Intersection with SR } 416 \\
\text { to Outside of Gatlinburg }\end{array}$ & $\begin{array}{r}1357 \\
\text { LOS=B }\end{array}$ & EB & $\begin{array}{l}38 \% \\
62 \%\end{array}$ & $5.00 \%$ \\
\hline Foothill 8B & From Cosby to Pittman Center & $\begin{array}{r}241 \\
\mathrm{LOS}=\mathrm{C}\end{array}$ & $\begin{array}{l}\mathrm{EB} \\
\mathrm{WB}\end{array}$ & $\begin{array}{l}55 \% \\
45 \%\end{array}$ & $0.00 \%$ \\
\hline
\end{tabular}

Table J.15. Weekend A.M. peak hour traffic projections for 2026-interchange at Highway 416 (both options) $-8 B$ not opened until $8 \mathrm{C}$ completed

\begin{tabular}{|c|c|c|c|c|c|}
\hline $\begin{array}{c}\text { Road section } \\
\text { name }\end{array}$ & Range & $\begin{array}{l}\text { Traffic } \\
\text { volume }\end{array}$ & Dir & split & $\begin{array}{c}\text { Percentage } \\
\text { of trucks }\end{array}$ \\
\hline U.S. 321 & $\begin{array}{l}\text { From Intersection with Foothills } \\
\text { Parkway Section } 8 \text { A to } \\
\text { Convergence with SR } 32\end{array}$ & $\begin{array}{r}1746 \\
\mathrm{LOS}=\mathrm{C}\end{array}$ & $\begin{array}{l}\text { NB } \\
\text { SB }\end{array}$ & $\begin{array}{l}51 \% \\
49 \%\end{array}$ & $9.00 \%$ \\
\hline U.S. 321 & $\begin{array}{l}\text { From U.S. } 321 \text { Convergence } \\
\text { with SR } 32 \text { to Intersection with } \\
\text { SR } 416\end{array}$ & $\begin{array}{r}1529 \\
\mathrm{LOS}=\mathrm{E}\end{array}$ & $\begin{array}{l}\text { WB } \\
\mathrm{EB}\end{array}$ & $\begin{array}{l}57 \% \\
43 \%\end{array}$ & $7.00 \%$ \\
\hline SR 416 & $\begin{array}{l}\text { From Intersection with U.S. } 321 \\
\text { to Intersection with Webb Creek } \\
\text { Rd }\end{array}$ & $\begin{array}{r}233 \\
\mathrm{LOS}=\mathrm{B}\end{array}$ & $\begin{array}{l}\text { NB } \\
\text { SB }\end{array}$ & $\begin{array}{l}64 \% \\
36 \%\end{array}$ & $7.00 \%$ \\
\hline U.S. 321 & $\begin{array}{l}\text { From Intersection with SR } 416 \\
\text { to Outside of Gatlinburg }\end{array}$ & $\begin{array}{r}1754 \\
\mathrm{LOS}=\mathrm{C}\end{array}$ & $\begin{array}{l}\text { EB } \\
\text { WB }\end{array}$ & $\begin{array}{l}33 \% \\
67 \%\end{array}$ & $5.00 \%$ \\
\hline Foothill 8B & From Cosby to Pittman Center & $\begin{array}{r}348 \\
\mathrm{LOS}=\mathrm{C}\end{array}$ & $\begin{array}{l}\mathrm{EB} \\
\mathrm{WB}\end{array}$ & $\begin{array}{l}60 \% \\
40 \% \\
\end{array}$ & $0.00 \%$ \\
\hline
\end{tabular}


Table J.16. Weekend P.M. peak hour traffic projections for 2006-interchange at Highway 416 (both options)-8B not opened until $8 \mathrm{C}$ completed

\begin{tabular}{llrrrr}
\hline $\begin{array}{c}\text { Road section } \\
\text { name }\end{array}$ & \multicolumn{1}{c}{ Range } & $\begin{array}{c}\text { Traffic } \\
\text { volume }\end{array}$ & Directional split & $\begin{array}{c}\text { Percentage } \\
\text { of trucks }\end{array}$ \\
\hline U.S. 321 & $\begin{array}{l}\text { From Intersection with Foothills } \\
\text { Parkway Section 8A to } \\
\text { Convergence with SR 32 }\end{array}$ & 1273 & NB & $55 \%$ & $9.00 \%$ \\
& LoS=B & SB & $45 \%$ & \\
U.S. 321 & $\begin{array}{l}\text { From U.S. 321 Convergence } \\
\text { with SR 32 to Intersection with }\end{array}$ & 1239 & WB & $57 \%$ & $7.00 \%$ \\
& SR 416 & LOS=D & EB & $43 \%$ & \\
SR 416 & $\begin{array}{l}\text { From Intersection with U.S. 321 } \\
\text { to Intersection with Webb Creek }\end{array}$ & 358 & NB & $74 \%$ & $7.00 \%$ \\
& Rd & LOS=C & SB & $26 \%$ & \\
U.S. 321 & From Intersection with SR 416 & 1342 & EB & $33 \%$ & $5.00 \%$ \\
& to Outside of Gatlinburg & LOS=B & WB & $67 \%$ & \\
Foothill 8B & From Cosby to Pittman Center & 264 & EB & $58 \%$ & $0.00 \%$ \\
& & LOS=C & WB & $42 \%$ & \\
\hline
\end{tabular}

Table J.17. Weekend P.M. peak hour traffic projections for 2026-interchange at Highway 416 (both options) $-8 \mathrm{~B}$ not opened until 8C completed

\begin{tabular}{|c|c|c|c|c|c|}
\hline $\begin{array}{c}\text { Road section } \\
\text { name }\end{array}$ & Range & $\begin{array}{l}\text { Traffic } \\
\text { volume }\end{array}$ & Di & split & $\begin{array}{l}\text { Percentage } \\
\text { of trucks }\end{array}$ \\
\hline U.S. 321 & $\begin{array}{l}\text { From Intersection with Foothills } \\
\text { Parkway Section } 8 \text { A to } \\
\text { Convergence with SR } 32\end{array}$ & $\begin{array}{r}1671 \\
\mathrm{LOS}=\mathrm{C}\end{array}$ & $\begin{array}{l}\text { NB } \\
\text { SB }\end{array}$ & $\begin{array}{l}54 \% \\
46 \%\end{array}$ & $9.00 \%$ \\
\hline U.S. 321 & $\begin{array}{l}\text { From U.S. } 321 \text { Convergence } \\
\text { with SR } 32 \text { to Intersection with } \\
\text { SR } 416\end{array}$ & $\begin{array}{r}1627 \\
\mathrm{LOS}=\mathrm{E}\end{array}$ & $\begin{array}{l}\text { WB } \\
\text { EB }\end{array}$ & $\begin{array}{l}56 \% \\
44 \%\end{array}$ & $7.00 \%$ \\
\hline SR 416 & $\begin{array}{l}\text { From Intersection with U.S. } 321 \\
\text { to Intersection with Webb Creek } \\
\text { Rd }\end{array}$ & $\begin{array}{r}360 \\
\mathrm{LOS}=\mathrm{C}\end{array}$ & $\begin{array}{l}\text { NB } \\
\text { SB }\end{array}$ & $\begin{array}{l}70 \% \\
30 \%\end{array}$ & $7.00 \%$ \\
\hline U.S. 321 & $\begin{array}{l}\text { From Intersection with SR } 416 \\
\text { to Outside of Gatlinburg }\end{array}$ & $\begin{array}{r}1788 \\
\operatorname{LOS}=C\end{array}$ & $\begin{array}{l}\text { EB } \\
\text { WB }\end{array}$ & $\begin{array}{l}31 \% \\
69 \%\end{array}$ & $5.00 \%$ \\
\hline Foothill 8B & From Cosby to Pittman Center & $\begin{array}{r}376 \\
\text { LOS }=C\end{array}$ & $\begin{array}{l}\text { EB } \\
\text { WB }\end{array}$ & $\begin{array}{l}64 \% \\
36 \% \\
\end{array}$ & $0.00 \%$ \\
\hline
\end{tabular}


Table J.18. Weekday A.M. peak hour traffic projections for 2006-interchange at U.S. 321 (both options)-8B not opened until 8C completed

\begin{tabular}{llrrrr}
\hline $\begin{array}{c}\text { Road section } \\
\text { name }\end{array}$ & \multicolumn{1}{c}{ Range } & $\begin{array}{c}\text { Traffic } \\
\text { volume }\end{array}$ & \multicolumn{1}{c}{ Directional split } & $\begin{array}{c}\text { Percentage } \\
\text { of trucks }\end{array}$ \\
\hline U.S. 321 & $\begin{array}{l}\text { From Intersection with Foothills } \\
\text { Parkway Section 8A to } \\
\text { Convergence with SR 32 }\end{array}$ & 950 & NB & $57 \%$ & $9.00 \%$ \\
U.S. 321 & $\begin{array}{l}\text { From U.S. 321 Convergence } \\
\text { with SR 32 to Intersection with }\end{array}$ & LOS=B & SB & $43 \%$ & WB \\
& SR 416 & LOS=D & EB & $54 \%$ & $7.00 \%$ \\
SR 416 & $\begin{array}{l}\text { From Intersection with U.S. 321 } \\
\text { to Intersection with Webb Creek }\end{array}$ & LOS=B & SB & $51 \%$ & \\
& Rd & NB & $49 \%$ & $7.00 \%$ \\
U.S. 321 & $\begin{array}{l}\text { From Intersection with SR 416 } \\
\text { to Outside of Gatlinburg }\end{array}$ & 1141 & EB & $36 \%$ & $5.00 \%$ \\
Foothill 8B & From Cosby to Pittman Center & LOS=B & WB & $64 \%$ & \\
& & LOS=B & WB & $48 \%$ & \\
\hline
\end{tabular}

Table J.19. Weekday A.M. peak hour traffic projections for 2026-interchange at U.S. 321 (both options)-8B not opened until $8 \mathrm{C}$ completed

\begin{tabular}{llrrrr}
\hline $\begin{array}{c}\text { Road section } \\
\text { name }\end{array}$ & \multicolumn{1}{c}{ Range } & $\begin{array}{c}\text { Traffic } \\
\text { volume }\end{array}$ & \multicolumn{1}{c}{ Directional split } & $\begin{array}{c}\text { Percentage } \\
\text { of trucks }\end{array}$ \\
\hline U.S. 321 & $\begin{array}{l}\text { From Intersection with Foothills } \\
\text { Parkway Section 8A to } \\
\text { Convergence with SR 32 }\end{array}$ & 1242 & NB & $56 \%$ & $9.00 \%$ \\
U.S. 321 & $\begin{array}{l}\text { From U.S. 321 Convergence } \\
\text { with SR 32 to Intersection with }\end{array}$ & 1086 & WB & $54 \%$ & $7.00 \%$ \\
& SR 416 & LOS=D & EB & $46 \%$ & \\
SR 416 & $\begin{array}{l}\text { From Intersection with U.S. 321 } \\
\text { to Intersection with Webb Creek }\end{array}$ & 248 & NB & $36 \%$ & $7.00 \%$ \\
Rd & LOS=B & SB & $64 \%$ & \\
U.S. 321 & $\begin{array}{l}\text { From Intersection with SR 416 } \\
\text { to Outside of Gatlinburg }\end{array}$ & 1503 & EB & $31 \%$ & $5.00 \%$ \\
Foothill 8B & From Cosby to Pittman Center & LOS=B & WB & $69 \%$ & \\
& & LOS=C & WB & $42 \%$ & \\
\hline
\end{tabular}


Table J.20. Weekday P.M. peak hour traffic projections for 2006-interchange at U.S. 321 (both options)-8B not opened until 8C completed

\begin{tabular}{llrrrr}
\hline $\begin{array}{c}\text { Road section } \\
\text { name }\end{array}$ & \multicolumn{1}{c}{ Range } & $\begin{array}{c}\text { Traffic } \\
\text { volume }\end{array}$ & Directional split & $\begin{array}{c}\text { Percentage } \\
\text { of trucks }\end{array}$ \\
\hline U.S. 321 & $\begin{array}{l}\text { From Intersection with Foothills } \\
\text { Parkway Section 8A to } \\
\text { Convergence with SR 32 }\end{array}$ & 1005 & NB & $59 \%$ & $9.00 \%$ \\
U.S. 321 & $\begin{array}{l}\text { From U.S. 321 Convergence } \\
\text { with SR 32 to Intersection with }\end{array}$ & 859 & WB & $51 \%$ & \\
& SR 416 & LOS=D & EB & $49 \%$ & $7.00 \%$ \\
SR 416 & $\begin{array}{l}\text { From Intersection with U.S. 321 } \\
\text { to Intersection with Webb Creek }\end{array}$ & 214 & NB & $56 \%$ & $7.00 \%$ \\
& Rd & LOS=B & SB & $44 \%$ & \\
U.S. 321 & $\begin{array}{l}\text { From Intersection with SR 416 } \\
\text { to Outside of Gatlinburg }\end{array}$ & 989 & EB & $32 \%$ & $5.00 \%$ \\
& & LOS=B & WB & $68 \%$ & \\
Foothill 8B & From Cosby to Pittman Center & 220 & EB & $58 \%$ & $0.00 \%$ \\
& & LOS=C & WB & $42 \%$ & \\
\hline
\end{tabular}

Table J.21. Weekday P.M. peak hour traffic projections for 2026-interchange at U.S. 321 (both options)-8B not opened until 8C completed

\begin{tabular}{|c|c|c|c|c|c|}
\hline \multirow{3}{*}{$\begin{array}{c}\begin{array}{c}\text { Road section } \\
\text { name }\end{array} \\
\text { U.S. } 321\end{array}$} & \multirow{3}{*}{$\begin{array}{l}\text { Range } \\
\text { From Intersection with Foothills } \\
\text { Parkway Section 8A to } \\
\text { Convergence with SR } 32\end{array}$} & \multirow{2}{*}{$\begin{array}{r}\begin{array}{r}\text { Traffic } \\
\text { volume }\end{array} \\
1316\end{array}$} & \multicolumn{2}{|c|}{ Directional split } & \multirow{3}{*}{$\begin{array}{r}\begin{array}{c}\text { Percentage } \\
\text { of trucks }\end{array} \\
9.00 \%\end{array}$} \\
\hline & & & NB & $59 \%$ & \\
\hline & & $\mathrm{LOS}=\mathrm{B}$ & SB & $41 \%$ & \\
\hline \multirow[t]{2}{*}{ U.S. 321} & \multirow{2}{*}{$\begin{array}{l}\text { From U.S. } 321 \text { Convergence } \\
\text { with SR } 32 \text { to Intersection with } \\
\text { SR } 416\end{array}$} & 1123 & WB & $50 \%$ & $7.00 \%$ \\
\hline & & $\mathrm{LOS}=\mathrm{D}$ & EB & $50 \%$ & \\
\hline \multirow[t]{2}{*}{ SR 416} & \multirow{2}{*}{$\begin{array}{l}\text { From Intersection with U.S. } 321 \\
\text { to Intersection with Webb Creek } \\
\text { Rd }\end{array}$} & 247 & NB & $44 \%$ & $7.00 \%$ \\
\hline & & $\mathrm{LOS}=\mathrm{B}$ & SB & $56 \%$ & \\
\hline \multirow[t]{2}{*}{ U.S. 321} & \multirow{2}{*}{$\begin{array}{l}\text { From Intersection with SR } 416 \\
\text { to Outside of Gatlinburg }\end{array}$} & 1332 & EB & $30 \%$ & $5.00 \%$ \\
\hline & & $\mathrm{LOS}=\mathrm{B}$ & WB & $70 \%$ & \\
\hline \multirow[t]{2}{*}{ Foothill 8B } & \multirow[t]{2}{*}{ From Cosby to Pittman Center } & 313 & EB & $64 \%$ & $0.00 \%$ \\
\hline & & $\mathrm{LOS}=\mathrm{C}$ & WB & $36 \%$ & \\
\hline
\end{tabular}


Table J.22. Weekend A.M. peak hour traffic projections for 2006-interchange at U.S. 321 (both options)-8B not opened until 8C completed

\begin{tabular}{|c|c|c|c|c|c|}
\hline \multirow{3}{*}{$\begin{array}{c}\begin{array}{c}\text { Road section } \\
\text { name }\end{array} \\
\text { U.S. } 321\end{array}$} & \multirow{3}{*}{$\begin{array}{l}\text { Range } \\
\text { From Intersection with Foothills } \\
\text { Parkway Section 8A to } \\
\text { Convergence with SR } 32\end{array}$} & \multirow{2}{*}{$\begin{array}{r}\begin{array}{r}\text { Traffic } \\
\text { volume }\end{array} \\
1332\end{array}$} & \multicolumn{2}{|c|}{ Directional split } & \multirow{3}{*}{$\begin{array}{r}\begin{array}{c}\text { Percentage } \\
\text { of trucks }\end{array} \\
9.00 \%\end{array}$} \\
\hline & & & NB & $52 \%$ & \\
\hline & & $\mathrm{LOS}=\mathrm{B}$ & SB & $48 \%$ & \\
\hline \multirow[t]{2}{*}{ U.S. 321} & \multirow{2}{*}{$\begin{array}{l}\text { From U.S. } 321 \text { Convergence } \\
\text { with SR } 32 \text { to Intersection with } \\
\text { SR } 416\end{array}$} & 1112 & WB & $55 \%$ & $7.00 \%$ \\
\hline & & $\mathrm{LOS}=\mathrm{D}$ & EB & $45 \%$ & \\
\hline \multirow[t]{2}{*}{ SR 416} & \multirow{2}{*}{$\begin{array}{l}\text { From Intersection with U.S. } 321 \\
\text { to Intersection with Webb Creek } \\
\text { Rd }\end{array}$} & 220 & NB & $55 \%$ & $7.00 \%$ \\
\hline & & LOS $=B$ & SB & $45 \%$ & \\
\hline \multirow[t]{2}{*}{ U.S. 321} & \multirow{2}{*}{$\begin{array}{l}\text { From Intersection with SR } 416 \\
\text { to Outside of Gatlinburg }\end{array}$} & 1395 & EB & $37 \%$ & $5.00 \%$ \\
\hline & & $\mathrm{LOS}=\mathrm{B}$ & WB & $63 \%$ & \\
\hline \multirow[t]{2}{*}{ Foothill 8B } & \multirow[t]{2}{*}{ From Cosby to Pittman Center } & 240 & EB & $55 \%$ & $0.00 \%$ \\
\hline & & $\mathrm{LOS}=\mathrm{C}$ & WB & $45 \%$ & \\
\hline
\end{tabular}

Table J.23. Weekend A.M. peak hour traffic projections for 2026-interchange at U.S. 321 (both options) $-8 \mathrm{~B}$ not opened until $8 \mathrm{C}$ completed

\begin{tabular}{|c|c|c|c|c|c|}
\hline $\begin{array}{c}\text { Road section } \\
\text { name }\end{array}$ & Range & $\begin{array}{l}\text { Traffic } \\
\text { volume }\end{array}$ & \multicolumn{2}{|c|}{ Directional split } & $\begin{array}{c}\text { Percentage } \\
\text { of trucks }\end{array}$ \\
\hline U.S. 321 & $\begin{array}{l}\text { From Intersection with Foothills } \\
\text { Parkway Section } 8 \text { A to } \\
\text { Convergence with SR } 32\end{array}$ & $\begin{array}{r}1746 \\
\mathrm{LOS}=\mathrm{C}\end{array}$ & $\begin{array}{l}\text { NB } \\
\text { SB }\end{array}$ & $\begin{array}{l}51 \% \\
49 \%\end{array}$ & $9.00 \%$ \\
\hline U.S. 321 & $\begin{array}{l}\text { From U.S. } 321 \text { Convergence } \\
\text { with SR } 32 \text { to Intersection with } \\
\text { SR } 416\end{array}$ & $\begin{array}{r}1491 \\
\mathrm{LOS}=\mathrm{E}\end{array}$ & $\begin{array}{l}\text { WB } \\
\text { EB }\end{array}$ & $\begin{array}{l}55 \% \\
45 \%\end{array}$ & $7.00 \%$ \\
\hline SR 416 & $\begin{array}{l}\text { From Intersection with U.S. } 321 \\
\text { to Intersection with Webb Creek } \\
\text { Rd }\end{array}$ & $\begin{array}{r}231 \\
\mathrm{LOS}=\mathrm{B}\end{array}$ & $\begin{array}{l}\text { NB } \\
\text { SB }\end{array}$ & $\begin{array}{l}38 \% \\
62 \%\end{array}$ & $7.00 \%$ \\
\hline U.S. 321 & $\begin{array}{l}\text { From Intersection with SR } 416 \\
\text { to Outside of Gatlinburg }\end{array}$ & $\begin{array}{r}1833 \\
\text { LOS }=\mathrm{C}\end{array}$ & $\begin{array}{l}\text { EB } \\
\text { WB }\end{array}$ & $\begin{array}{l}33 \% \\
67 \%\end{array}$ & $5.00 \%$ \\
\hline Foothill 8B & From Cosby to Pittman Center & $\begin{array}{r}348 \\
L O S=C\end{array}$ & $\begin{array}{l}\text { EB } \\
\text { WB }\end{array}$ & $\begin{array}{l}60 \% \\
40 \% \\
\end{array}$ & $0.00 \%$ \\
\hline
\end{tabular}


Table J.24. Weekend P.M. peak hour traffic projections for 2006-interchange at U.S. 321 (both options)-8B not opened until 8C completed

\begin{tabular}{llrrrr}
\hline $\begin{array}{c}\text { Road section } \\
\text { name }\end{array}$ & \multicolumn{1}{c}{ Range } & $\begin{array}{c}\text { Traffic } \\
\text { volume }\end{array}$ & Directional split & $\begin{array}{c}\text { Percentage } \\
\text { of trucks }\end{array}$ \\
\hline U.S. 321 & $\begin{array}{l}\text { From Intersection with Foothills } \\
\text { Parkway Section 8A to } \\
\text { Convergence with SR 32 }\end{array}$ & 1272 & NB & $55 \%$ & $9.00 \%$ \\
& LOS=B & SB & $45 \%$ & \\
U.S. 321 & $\begin{array}{l}\text { From U.S. 321 Convergence } \\
\text { with SR 32 to Intersection with }\end{array}$ & 1174 & WB & $54 \%$ & $7.00 \%$ \\
& SR 416 & LOS=D & EB & $46 \%$ & \\
SR 416 & $\begin{array}{l}\text { From Intersection with U.S. 321 } \\
\text { to Intersection with Webb Creek }\end{array}$ & 282 & NB & $52 \%$ & $7.00 \%$ \\
& Rd & LOS=B & SB & $48 \%$ & \\
U.S. 321 & $\begin{array}{l}\text { From Intersection with SR 416 } \\
\text { to Outside of Gatlinburg }\end{array}$ & 1384 & EB & $33 \%$ & $5.00 \%$ \\
& From Cosby to Pittman Center & 263 & EB & $58 \%$ & $0.00 \%$ \\
\hline
\end{tabular}

Table J.25. Weekend P.M. peak hour traffic projections for 2026-interchange at U.S. 321 (both options) -8B not opened until 8C completed

\begin{tabular}{llrrrr}
\hline $\begin{array}{c}\text { Road section } \\
\text { name }\end{array}$ & \multicolumn{1}{c}{ Range } & $\begin{array}{c}\text { Traffic } \\
\text { volume }\end{array}$ & Directional split & $\begin{array}{c}\text { Percentage } \\
\text { of trucks }\end{array}$ \\
\hline U.S. 321 & $\begin{array}{l}\text { From Intersection with Foothills } \\
\text { Parkway Section 8A to }\end{array}$ & 1671 & NB & $54 \%$ & $9.00 \%$ \\
& $\begin{array}{l}\text { Convergence with SR 32 } \\
\text { U.S.321 }\end{array}$ & LOS=C & SB & $46 \%$ & \\
& $\begin{array}{l}\text { From U.S. 321 Convergence } \\
\text { with SR 32 to Intersection with }\end{array}$ & 1538 & WB & $53 \%$ & $7.00 \%$ \\
SR 416 & LOS=E & EB & $47 \%$ & \\
& $\begin{array}{l}\text { From Intersection with U.S. 321 } \\
\text { to Intersection with Webb Creek }\end{array}$ & 316 & NB & $38 \%$ & $7.00 \%$ \\
U.S. 321 & Rd & FoS=B & SB & $62 \%$ & \\
& $\begin{array}{l}\text { From Intersection with SR 416 } \\
\text { to Outside of Gatlinburg }\end{array}$ & 1871 & EB & $30 \%$ & $5.00 \%$ \\
Foothill 8B & From Cosby to Pittman Center & LOS=C & WB & $70 \%$ & \\
& & LOS=C & WB & $376 \%$ & \\
\hline
\end{tabular}


Table J.26. Weekday A.M. peak hour traffic projections for 2006-interchange at Highway 416 (both options)-8B opened prior to completion of 8C

\begin{tabular}{|c|c|c|c|c|c|}
\hline \multirow{2}{*}{$\begin{array}{c}\begin{array}{c}\text { Road section } \\
\text { name }\end{array} \\
\text { U.S. } 321\end{array}$} & Range & \multirow{2}{*}{$\begin{array}{r}\begin{array}{r}\text { Traffic } \\
\text { volume }\end{array} \\
950\end{array}$} & \multicolumn{2}{|c|}{ Directional split } & \multirow{3}{*}{$\begin{array}{r}\begin{array}{c}\text { Percentage } \\
\text { of trucks }\end{array} \\
9.00 \%\end{array}$} \\
\hline & From Intersection with Foothills & & NB & $57 \%$ & \\
\hline & $\begin{array}{l}\text { Parkway Section } 8 \mathrm{~A} \text { to } \\
\text { Convergence with SR } 32\end{array}$ & $\mathrm{LOS}=\mathrm{B}$ & SB & $43 \%$ & \\
\hline \multirow[t]{2}{*}{ U.S. 321} & \multirow{2}{*}{$\begin{array}{l}\text { From U.S. } 321 \text { Convergence } \\
\text { with SR } 32 \text { to Intersection with } \\
\text { SR } 416\end{array}$} & 834 & WB & $55 \%$ & $7.00 \%$ \\
\hline & & $\mathrm{LOS}=\mathrm{D}$ & EB & $45 \%$ & \\
\hline \multirow[t]{2}{*}{ SR 416} & \multirow{2}{*}{$\begin{array}{l}\text { From Intersection with U.S. } 321 \\
\text { to Intersection with Webb Creek } \\
\text { Rd }\end{array}$} & 364 & NB & $56 \%$ & $7.00 \%$ \\
\hline & & $\operatorname{LOS}=\mathrm{B}$ & SB & $44 \%$ & \\
\hline \multirow[t]{2}{*}{ U.S. 321} & \multirow{2}{*}{$\begin{array}{l}\text { From Intersection with SR } 416 \\
\text { to Outside of Gatlinburg }\end{array}$} & 1414 & EB & $37 \%$ & $5.00 \%$ \\
\hline & & $\mathrm{LOS}=\mathrm{B}$ & WB & $63 \%$ & \\
\hline \multirow[t]{2}{*}{ Foothill 8B } & \multirow[t]{2}{*}{ From Cosby to Pittman Center } & 185 & EB & $52 \%$ & $0.00 \%$ \\
\hline & & $\mathrm{LOS}=\mathrm{B}$ & WB & $48 \%$ & \\
\hline
\end{tabular}

Table J.27. Weekday A.M. peak hour traffic projections for 2026-interchange at Highway 416 (both options) - $8 B$ opened prior to completion of $8 \mathrm{C}$

\begin{tabular}{|c|c|c|c|c|c|}
\hline \multirow{2}{*}{$\begin{array}{c}\begin{array}{c}\text { Road section } \\
\text { name }\end{array} \\
\text { U.S. } 321\end{array}$} & Range & \multirow{2}{*}{$\begin{array}{r}\begin{array}{r}\text { Traffic } \\
\text { volume }\end{array} \\
1242\end{array}$} & \multicolumn{2}{|c|}{ Directional split } & \multirow{3}{*}{$\begin{array}{r}\begin{array}{c}\text { Percentage } \\
\text { of trucks }\end{array} \\
9.00 \%\end{array}$} \\
\hline & From Intersection with Foothills & & NB & $56 \%$ & \\
\hline & Convergence with SR 32 & $\mathrm{LOS}=\mathrm{B}$ & SB & $44 \%$ & \\
\hline \multirow[t]{2}{*}{ U.S. 321} & \multirow{2}{*}{$\begin{array}{l}\text { From U.S. } 321 \text { Convergence } \\
\text { with SR } 32 \text { to Intersection with } \\
\text { SR } 416\end{array}$} & 1117 & WB & $55 \%$ & $7.00 \%$ \\
\hline & & $\mathrm{LOS}=\mathrm{D}$ & EB & $45 \%$ & \\
\hline \multirow[t]{2}{*}{ SR 416} & \multirow{2}{*}{$\begin{array}{l}\text { From Intersection with U.S. } 321 \\
\text { to Intersection with Webb Creek } \\
\text { Rd }\end{array}$} & 469 & NB & $52 \%$ & $7.00 \%$ \\
\hline & & $\mathrm{LOS}=\mathrm{C}$ & SB & $48 \%$ & \\
\hline \multirow[t]{2}{*}{ U.S. 321} & \multirow{2}{*}{$\begin{array}{l}\text { From Intersection with SR } 416 \\
\text { to Outside of Gatlinburg }\end{array}$} & 1861 & EB & $34 \%$ & $5.00 \%$ \\
\hline & & $\operatorname{LOS}=\mathrm{C}$ & WB & $66 \%$ & \\
\hline \multirow[t]{2}{*}{ Foothill $8 \mathrm{~B}$} & \multirow[t]{2}{*}{ From Cosby to Pittman Center } & 263 & EB & $58 \%$ & $0.00 \%$ \\
\hline & & $\mathrm{LOS}=\mathrm{C}$ & WB & $42 \%$ & \\
\hline
\end{tabular}


Table J.28. Weekday P.M. peak hour traffic projections for 2006-interchange at Highway 416 (both options) $-8 \mathrm{~B}$ opened prior to completion of $8 \mathrm{C}$

\begin{tabular}{llrrrr}
\hline $\begin{array}{c}\text { Road section } \\
\text { name }\end{array}$ & \multicolumn{1}{c}{ Range } & $\begin{array}{c}\text { Traffic } \\
\text { volume }\end{array}$ & Directional split & $\begin{array}{c}\text { Percentage } \\
\text { of trucks }\end{array}$ \\
\hline U.S. 321 & $\begin{array}{l}\text { From Intersection with Foothills } \\
\text { Parkway Section 8A to } \\
\text { Convergence with SR 32 }\end{array}$ & 1005 & NB & $59 \%$ & $9.00 \%$ \\
U.S. 321 & $\begin{array}{l}\text { From U.S. 321 Convergence } \\
\text { with SR 32 to Intersection with }\end{array}$ & 873 & WB & $52 \%$ & $7.00 \%$ \\
& SR 416 & LOS=D & EB & $48 \%$ & \\
SR 416 & $\begin{array}{l}\text { From Intersection with U.S. 321 } \\
\text { to Intersection with Webb Creek }\end{array}$ & 329 & NB & $61 \%$ & $7.00 \%$ \\
& Rd & LOS=B & SB & $39 \%$ & \\
U.S. 321 & From Intersection with SR 416 & 1175 & EB & $32 \%$ & $5.00 \%$ \\
& to Outside of Gatlinburg & LOS=B & WB & $68 \%$ & \\
Foothill 8B & From Cosby to Pittman Center & 220 & EB & $58 \%$ & $0.00 \%$ \\
& & LOS=C & WB & $42 \%$ & \\
\hline
\end{tabular}

Table J.29. Weekday P.M. peak hour traffic projections for 2026-interchange at Highway 416 (both options) $-8 \mathrm{~B}$ opened prior to completion of $8 \mathrm{C}$

\begin{tabular}{|c|c|c|c|c|c|}
\hline \multirow{2}{*}{$\begin{array}{c}\begin{array}{c}\text { Road section } \\
\text { name }\end{array} \\
\text { U.S. } 321\end{array}$} & Range & \multirow{2}{*}{$\begin{array}{r}\begin{array}{r}\text { Traffic } \\
\text { volume }\end{array} \\
1316\end{array}$} & \multicolumn{2}{|c|}{ Directional split } & \multirow{3}{*}{$\begin{array}{r}\begin{array}{c}\text { Percentage } \\
\text { of trucks }\end{array} \\
9.00 \%\end{array}$} \\
\hline & From Intersection with Foothills & & NB & $59 \%$ & \\
\hline & $\begin{array}{l}\text { Parkway Section } 8 A \text { to } \\
\text { Convergence with SR } 32\end{array}$ & $\mathrm{LOS}=\mathrm{B}$ & SB & $41 \%$ & \\
\hline \multirow[t]{2}{*}{ U.S. 321} & \multirow{2}{*}{$\begin{array}{l}\text { From U.S. } 321 \text { Convergence } \\
\text { with SR } 32 \text { to Intersection with } \\
\text { SR } 416\end{array}$} & 1151 & WB & $52 \%$ & $7: 00 \%$ \\
\hline & & $\mathrm{LOS}=\mathrm{D}$ & EB & $48 \%$ & \\
\hline \multirow[t]{2}{*}{ SR 416} & \multirow{2}{*}{$\begin{array}{l}\text { From Intersection with U.S. } 321 \\
\text { to Intersection with Webb Creek } \\
\text { Rd }\end{array}$} & 433 & NB & $58 \%$ & $7.00 \%$ \\
\hline & & $\operatorname{LOS}=\mathrm{C}$ & SB & $42 \%$ & \\
\hline \multirow[t]{2}{*}{ U.S. 321} & \multirow{2}{*}{$\begin{array}{l}\text { From Intersection with SR } 416 \\
\text { to Outside of Gatlinburg }\end{array}$} & 1570 & $\mathrm{~EB}$ & $31 \%$ & $5.00 \%$ \\
\hline & & $\operatorname{LOS}=\mathrm{C}$ & WB & $69 \%$ & \\
\hline \multirow[t]{2}{*}{ Foothill 8B } & \multirow[t]{2}{*}{ From Cosby to Pittman Center } & 313 & EB & $64 \%$ & $0.00 \%$ \\
\hline & & $\mathrm{LOS}=\mathrm{C}$ & WB & $36 \%$ & \\
\hline
\end{tabular}


Table J.30. Weekend A.M. peak hour traffic projections for 2006-interchange at Highway 416 (both options) $-8 \mathrm{~B}$ opened prior to completion of $8 \mathrm{C}$

\begin{tabular}{|c|c|c|c|c|c|}
\hline $\begin{array}{l}\text { Road section } \\
\text { name }\end{array}$ & Range & $\begin{array}{l}\text { Traffic } \\
\text { volume }\end{array}$ & Dir & split & $\begin{array}{l}\text { Percentage } \\
\text { of trucks }\end{array}$ \\
\hline U.S. 321 & $\begin{array}{l}\text { From Intersection with Foothills } \\
\text { Parkway Section } 8 \text { A to } \\
\text { Convergence with SR } 32\end{array}$ & $\begin{array}{r}1332 \\
\operatorname{LOS}=\mathrm{B}\end{array}$ & $\begin{array}{l}\text { NB } \\
\text { SB }\end{array}$ & $\begin{array}{l}52 \% \\
48 \%\end{array}$ & $9.00 \%$ \\
\hline U.S. 321 & $\begin{array}{l}\text { From U.S. } 321 \text { Convergence } \\
\text { with SR } 32 \text { to Intersection with } \\
\text { SR } 416\end{array}$ & $\begin{array}{r}1139 \\
\mathrm{LOS}=\mathrm{D}\end{array}$ & $\begin{array}{l}\text { WB } \\
\text { EB }\end{array}$ & $\begin{array}{l}57 \% \\
43 \%\end{array}$ & $7.00 \%$ \\
\hline SR 416 & $\begin{array}{l}\text { From Intersection with U.S. } 321 \\
\text { to Intersection with Webb Creek } \\
\text { Rd }\end{array}$ & $\begin{array}{r}405 \\
\mathrm{LOS}=\mathrm{C}\end{array}$ & $\begin{array}{l}\text { NB } \\
\text { SB }\end{array}$ & $\begin{array}{l}62 \% \\
38 \%\end{array}$ & $7.00 \%$ \\
\hline U.S. 321 & $\begin{array}{l}\text { From Intersection with SR } 416 \\
\text { to Outside of Gatlinburg }\end{array}$ & $\begin{array}{r}1723 \\
\text { LOS }=\mathrm{C}\end{array}$ & $\begin{array}{l}\text { EB } \\
\text { WB }\end{array}$ & $\begin{array}{l}38 \% \\
62 \%\end{array}$ & $5.00 \%$ \\
\hline Foothill 8B & From Cosby to Pittman Center & $\begin{array}{r}240 \\
\mathrm{LOS}=\mathrm{C}\end{array}$ & $\begin{array}{l}\text { EB } \\
\text { WB }\end{array}$ & $\begin{array}{l}55 \% \\
45 \%\end{array}$ & $0.00 \%$ \\
\hline
\end{tabular}

Table J.31. Weekend A.M. peak hour traffic projections for 2026-interchange at Highway 416 (both options)-8B opened prior to completion of $8 \mathrm{C}$

\begin{tabular}{|c|c|c|c|c|c|}
\hline $\begin{array}{c}\text { Road section } \\
\text { name }\end{array}$ & Range & $\begin{array}{l}\text { Traffic } \\
\text { volume }\end{array}$ & \multicolumn{2}{|c|}{ Directional split } & $\begin{array}{c}\text { Percentage } \\
\text { of trucks }\end{array}$ \\
\hline U.S. 321 & $\begin{array}{l}\text { From Intersection with Foothills } \\
\text { Parkway Section } 8 \text { A to } \\
\text { Convergence with SR } 32\end{array}$ & $\begin{array}{r}1746 \\
\mathrm{LOS}=\mathrm{C}\end{array}$ & NB & $\begin{array}{l}51 \% \\
49 \%\end{array}$ & $9.00 \%$ \\
\hline U.S. 321 & $\begin{array}{l}\text { From U.S. } 321 \text { Convergence } \\
\text { with SR } 32 \text { to Intersection with } \\
\text { SR } 416\end{array}$ & $\begin{array}{r}1529 \\
\text { LOS }=E\end{array}$ & $\begin{array}{l}\text { WB } \\
\text { EB }\end{array}$ & $\begin{array}{l}57 \% \\
43 \%\end{array}$ & $7.00 \%$ \\
\hline SR 416 & $\begin{array}{l}\text { From Intersection with U.S. } 321 \\
\text { to Intersection with Webb Creek } \\
\text { Rd }\end{array}$ & $\begin{array}{r}509 \\
\mathrm{LOS}=\mathrm{C}\end{array}$ & $\begin{array}{l}\text { NB } \\
\text { SB }\end{array}$ & $\begin{array}{l}58 \% \\
42 \%\end{array}$ & $7.00 \%$ \\
\hline U.S. 321 & $\begin{array}{l}\text { From Intersection with SR } 416 \\
\text { to Outside of Gatlinburg }\end{array}$ & $\begin{array}{r}2263 \\
\text { LOS }=D\end{array}$ & EB & $\begin{array}{l}36 \% \\
64 \%\end{array}$ & $5.00 \%$ \\
\hline Foothill 8B & From Cosby to Pittman Center & $\begin{array}{r}348 \\
\text { LOS }=C\end{array}$ & $\begin{array}{l}\text { EB } \\
\text { WB }\end{array}$ & $\begin{array}{l}60 \% \\
40 \%\end{array}$ & $0.00 \%$ \\
\hline
\end{tabular}


Table J.32. Weekend P.M. peak hour traffic projections for 2006-interchange at Highway 416 (both options) $-8 B$ opened prior to completion of $8 \mathrm{C}$

\begin{tabular}{llrrrr}
$\begin{array}{c}\text { Road section } \\
\text { name }\end{array}$ & \multicolumn{1}{c}{ Range } & $\begin{array}{c}\text { Traffic } \\
\text { volume }\end{array}$ & \multicolumn{1}{c}{ Directional split } & $\begin{array}{c}\text { Percentage } \\
\text { of trucks }\end{array}$ \\
\hline U.S. 321 & $\begin{array}{l}\text { From Intersection with Foothills } \\
\text { Parkway Section 8A to } \\
\text { Convergence with SR 32 }\end{array}$ & 1272 & NB & $55 \%$ & $9.00 \%$ \\
U.S. 321 & $\begin{array}{l}\text { From U.S. 321 Convergence } \\
\text { with SR 32 to Intersection with }\end{array}$ & 1192 & WB & $55 \%$ & $7.00 \%$ \\
& SR 416 & LOS=D & EB & $45 \%$ & \\
SR 416 & $\begin{array}{l}\text { From Intersection with U.S. 321 } \\
\text { to Intersection with Webb Creek }\end{array}$ & 449 & NB & $55 \%$ & $7.00 \%$ \\
Rd & LOS=C & SB & $45 \%$ & \\
U.S. 321 & $\begin{array}{l}\text { From Intersection with SR 416 } \\
\text { to Outside of Gatlinburg }\end{array}$ & 1677 & EB & $32 \%$ & $5.00 \%$ \\
Foothill 8B & From Cosby to Pittman Center & LOS=C & WB & $68 \%$ & \\
& & LOS=C & WB & $42 \%$ & \\
\hline
\end{tabular}

Table J.33. Weekend P.M. peak hour traffic projections for 2026-interchange at Highway 416 (both options)-8B opened prior to completion of $8 \mathrm{C}$

\begin{tabular}{|c|c|c|c|c|c|}
\hline $\begin{array}{c}\text { Road section } \\
\text { name }\end{array}$ & Range & $\begin{array}{l}\text { Traffic } \\
\text { volume }\end{array}$ & & split & $\begin{array}{c}\text { Percentage } \\
\text { of trucks }\end{array}$ \\
\hline U.S. 321 & $\begin{array}{l}\text { From Intersection with Foothills } \\
\text { Parkway Section } 8 \text { A to } \\
\text { Convergence with SR } 32\end{array}$ & $\begin{array}{r}1671 \\
\mathrm{LOS}=\mathrm{C}\end{array}$ & $\begin{array}{l}\text { NB } \\
\text { SB }\end{array}$ & $\begin{array}{l}54 \% \\
46 \%\end{array}$ & $9.00 \%$ \\
\hline U.S. 321 & $\begin{array}{l}\text { From U.S. } 321 \text { Convergence } \\
\text { with SR } 32 \text { to Intersection with } \\
\text { SR } 416\end{array}$ & $\begin{array}{r}1577 \\
\mathrm{LOS}=\mathrm{E}\end{array}$ & WB & $\begin{array}{l}55 \% \\
45 \%\end{array}$ & $7.00 \%$ \\
\hline SR 416 & $\begin{array}{l}\text { From Intersection with U.S. } 321 \\
\text { to Intersection with Webb Creek } \\
\text { Rd }\end{array}$ & $\begin{array}{r}581 \\
\mathrm{LOS}=\mathrm{C}\end{array}$ & NB & $\begin{array}{l}52 \% \\
48 \%\end{array}$ & $7.00 \%$ \\
\hline U.S. 321 & $\begin{array}{l}\text { From Intersection with SR } 416 \\
\text { to Outside of Gatlinburg }\end{array}$ & $\begin{array}{r}2246 \\
\text { LOS }=D\end{array}$ & WB & $\begin{array}{l}30 \% \\
70 \%\end{array}$ & $5.00 \%$ \\
\hline Foothill 8B & From Cosby to Pittman Center & $\begin{array}{r}376 \\
\mathrm{LOS}=\mathrm{C}\end{array}$ & $\begin{array}{l}\text { EB } \\
\text { WB }\end{array}$ & $\begin{array}{l}64 \% \\
36 \%\end{array}$ & $0.00 \%$ \\
\hline
\end{tabular}


Table J.34. Weekday A.M. peak hour traffic projections for 2006-interchange at U.S. 321 (both options)-8B opened prior to completion of $8 \mathrm{C}$

\begin{tabular}{|c|c|c|c|c|c|}
\hline $\begin{array}{c}\text { Road section } \\
\text { name }\end{array}$ & Range & $\begin{array}{l}\text { Traffic } \\
\text { volume }\end{array}$ & Dir & plit & $\begin{array}{l}\text { Percentage } \\
\text { of trucks }\end{array}$ \\
\hline U.S. 321 & $\begin{array}{l}\text { From Intersection with Foothills } \\
\text { Parkway Section } 8 \mathrm{~A} \text { to } \\
\text { Convergence with SR } 32\end{array}$ & $\begin{array}{r}950 \\
\mathrm{LOS}=\mathrm{B}\end{array}$ & $\begin{array}{l}\mathrm{NB} \\
\mathrm{SB}\end{array}$ & $\begin{array}{l}57 \% \\
43 \%\end{array}$ & $9.00 \%$ \\
\hline U.S. 321 & $\begin{array}{l}\text { From U.S. } 321 \text { Convergence } \\
\text { with SR } 32 \text { to Intersection with } \\
\text { SR } 416\end{array}$ & $\begin{array}{r}930 \\
\mathrm{LOS}=\mathrm{D}\end{array}$ & WB & $\begin{array}{l}54 \% \\
46 \%\end{array}$ & $7.00 \%$ \\
\hline SR 416 & $\begin{array}{l}\text { From Intersection with U.S. } 321 \\
\text { to Intersection with Webb Creek } \\
\text { Rd }\end{array}$ & $\begin{array}{r}214 \\
\mathrm{LOS}=\mathrm{B}\end{array}$ & NB & $\begin{array}{l}49 \% \\
51 \%\end{array}$ & $7.00 \%$ \\
\hline U.S. 321 & $\begin{array}{l}\text { From Intersection with SR } 416 \\
\text { to Outside of Gatlinburg }\end{array}$ & $\begin{array}{r}1404 \\
\text { LOS=B }\end{array}$ & $\begin{array}{l}\text { EB } \\
\text { WB }\end{array}$ & $\begin{array}{l}37 \% \\
63 \%\end{array}$ & $5.00 \%$ \\
\hline Foothill 8B & From Cosby to Pittman Center & $\begin{array}{r}185 \\
\text { LOS }=B\end{array}$ & $\begin{array}{l}\text { EB } \\
\text { WB }\end{array}$ & $\begin{array}{l}52 \% \\
48 \% \\
\end{array}$ & $0.00 \%$ \\
\hline
\end{tabular}

Table J.35. Weekday A.M. peak hour traffic projections for 2026-interchange at U.S. 321 (both options)-8B opened prior to completion of $8 \mathrm{C}$

\begin{tabular}{|c|c|c|c|c|c|}
\hline $\begin{array}{c}\text { Road section } \\
\text { name }\end{array}$ & Range & $\begin{array}{c}\text { Traffic } \\
\text { volume } \\
\end{array}$ & \multicolumn{2}{|c|}{ Directional split } & $\begin{array}{c}\text { Percentage } \\
\text { of trucks }\end{array}$ \\
\hline U.S. 321 & $\begin{array}{l}\text { From Intersection with Foothills } \\
\text { Parkway Section } 8 \text { A to } \\
\text { Convergence with SR } 32\end{array}$ & $\begin{array}{r}1242 \\
\mathrm{LOS}=\mathrm{B}\end{array}$ & SB & $\begin{array}{l}56 \% \\
44 \%\end{array}$ & $9.00 \%$ \\
\hline U.S. 321 & $\begin{array}{l}\text { From U.S. } 321 \text { Convergence } \\
\text { with SR } 32 \text { to Intersection with } \\
\text { SR } 416\end{array}$ & $\begin{array}{r}1257 \\
\mathrm{LOS}=\mathrm{D}\end{array}$ & WB & $\begin{array}{l}53 \% \\
47 \%\end{array}$ & $7.00 \%$ \\
\hline SR 416 & $\begin{array}{l}\text { From Intersection with U.S. } 321 \\
\text { to Intersection with Webb Creek } \\
\text { Rd }\end{array}$ & $\begin{array}{r}247 \\
\mathrm{LOS}=\mathrm{B}\end{array}$ & NB & $\begin{array}{l}36 \% \\
64 \%\end{array}$ & $7.00 \%$ \\
\hline U.S. 321 & $\begin{array}{l}\text { From Intersection with SR } 416 \\
\text { to Outside of Gatlinburg }\end{array}$ & $\begin{array}{r}1850 \\
\operatorname{LOS}=\mathrm{C}\end{array}$ & WB & $\begin{array}{l}34 \% \\
66 \%\end{array}$ & $5.00 \%$ \\
\hline Foothill 8B & From Cosby to Pittman Center & $\begin{array}{r}263 \\
\mathrm{LOS}=\mathrm{C}\end{array}$ & $\begin{array}{l}\text { EB } \\
\text { WB }\end{array}$ & $\begin{array}{l}58 \% \\
42 \% \\
\end{array}$ & $0.00 \%$ \\
\hline
\end{tabular}


Table J.36. Weekday P.M. peak hour traffic projections for 2006-interchange at U.S. 321 (both options)-8B opened prior to completion of $8 \mathrm{C}$

\begin{tabular}{|c|c|c|c|c|c|}
\hline \multirow{2}{*}{$\begin{array}{c}\begin{array}{c}\text { Road section } \\
\text { name }\end{array} \\
\text { U.S. } 321\end{array}$} & Range & \multirow{2}{*}{$\begin{array}{r}\begin{array}{r}\text { Traffic } \\
\text { volume }\end{array} \\
1005\end{array}$} & \multicolumn{2}{|c|}{ Directional split } & \multirow{3}{*}{$\begin{array}{r}\begin{array}{r}\text { Percentage } \\
\text { of trucks }\end{array} \\
9.00 \%\end{array}$} \\
\hline & From Intersection with Foothills & & NB & $59 \%$ & \\
\hline & $\begin{array}{l}\text { Parkway Section } 8 \mathrm{~A} \text { to } \\
\text { Convergence with SR } 32\end{array}$ & $\mathrm{LOS}=\mathrm{B}$ & SB & $41 \%$ & \\
\hline \multirow[t]{2}{*}{ U.S. 321} & \multirow{2}{*}{$\begin{array}{l}\text { From U.S. } 321 \text { Convergence } \\
\text { with SR } 32 \text { to Intersection with } \\
\text { SR } 416\end{array}$} & 961 & WB & $52 \%$ & $7.00 \%$ \\
\hline & & $\mathrm{LOS}=\mathrm{D}$ & EB & $48 \%$ & \\
\hline \multirow[t]{2}{*}{ SR 416} & \multirow{2}{*}{$\begin{array}{l}\text { From Intersection with U.S. } 321 \\
\text { to Intersection with Webb Creek } \\
\text { Rd }\end{array}$} & 204 & NB & $54 \%$ & $7.00 \%$ \\
\hline & & $\mathrm{LOS}=\mathrm{B}$ & SB & $46 \%$ & \\
\hline \multirow[t]{2}{*}{ U.S. 321} & \multirow{2}{*}{$\begin{array}{l}\text { From Intersection with SR } 416 \\
\text { to Outside of Gatlinburg }\end{array}$} & 1226 & $\mathrm{~EB}$ & $34 \%$ & $5.00 \%$ \\
\hline & & $\mathrm{LOS}=\mathrm{B}$ & WB & $66 \%$ & \\
\hline \multirow[t]{2}{*}{ Foothill 8B } & \multirow[t]{2}{*}{ From Cosby to Pittman Center } & 220 & EB & $58 \%$ & $0.00 \%$ \\
\hline & & $\mathrm{LOS}=\mathrm{C}$ & WB & $42 \%$ & . \\
\hline
\end{tabular}

Table J.37. Weekday P.M. peak hour traffic projections for 2026-interchange at U.S. 321 (both options)-8B opened prior to completion of 8C

\begin{tabular}{|c|c|c|c|c|c|}
\hline $\begin{array}{c}\text { Road section } \\
\text { name }\end{array}$ & Range & $\begin{array}{l}\text { Traffic } \\
\text { volume } \\
\end{array}$ & Dir & plit & $\begin{array}{c}\text { Percentage } \\
\text { of trucks }\end{array}$ \\
\hline U.S. 321 & $\begin{array}{l}\text { From Intersection with Foothills } \\
\text { Parkway Section } 8 \text { A to } \\
\text { Convergence with SR } 32\end{array}$ & $\begin{array}{r}1316 \\
\text { LOS=B }\end{array}$ & $\begin{array}{l}\text { NB } \\
\text { SB }\end{array}$ & $\begin{array}{l}59 \% \\
41 \%\end{array}$ & $9.00 \%$ \\
\hline U.S. 321 & $\begin{array}{l}\text { From U.S. } 321 \text { Convergence } \\
\text { with SR } 32 \text { to Intersection with } \\
\text { SR } 416\end{array}$ & $\begin{array}{r}1277 \\
\text { LOS }=D\end{array}$ & $\begin{array}{l}\text { WB } \\
\text { EB }\end{array}$ & $\begin{array}{l}51 \% \\
49 \%\end{array}$ & $7.00 \%$ \\
\hline SR 416 & $\begin{array}{l}\text { From Intersection with U.S. } 321 \\
\text { to Intersection with Webb Creek } \\
\text { Rd }\end{array}$ & $\begin{array}{r}245 \\
\text { LOS }=B\end{array}$ & $\begin{array}{l}\text { NB } \\
\text { SB }\end{array}$ & $\begin{array}{l}43 \% \\
57 \%\end{array}$ & $7.00 \%$ \\
\hline U.S. 321 & $\begin{array}{l}\text { From Intersection with SR } 416 \\
\text { to Outside of Gatlinburg }\end{array}$ & $\begin{array}{r}1645 \\
\mathrm{LOS}=\mathrm{C}\end{array}$ & WB & $\begin{array}{l}33 \% \\
67 \%\end{array}$ & $5.00 \%$ \\
\hline Foothill 8B & From Cosby to Pittman Center & $\begin{array}{r}313 \\
\mathrm{LOS}=\mathrm{C}\end{array}$ & $\begin{array}{l}\text { EB } \\
\text { WB }\end{array}$ & $\begin{array}{l}64 \% \\
36 \%\end{array}$ & $0.00 \%$ \\
\hline
\end{tabular}


Table J.38. Weekend A.M. peak hour traffic projections for 2006-interchange at U.S. 321 (both options)-8B opened prior to completion of $8 \mathrm{C}$

\begin{tabular}{llrrrr}
\hline $\begin{array}{c}\text { Road section } \\
\text { name }\end{array}$ & \multicolumn{1}{c}{ Range } & $\begin{array}{c}\text { Traffic } \\
\text { volume }\end{array}$ & Directional split & $\begin{array}{c}\text { Percentage } \\
\text { of trucks }\end{array}$ \\
\hline U.S. 321 & $\begin{array}{l}\text { From Intersection with Foothills } \\
\text { Parkway Section 8A to } \\
\text { Convergence with SR 32 }\end{array}$ & 1332 & NB & $52 \%$ & $9.00 \%$ \\
U.S. 321 & $\begin{array}{l}\text { From U.S. 321 Convergence } \\
\text { with SR 32 to Intersection with }\end{array}$ & 1259 & WB & $48 \%$ & \\
& SR 416 & LOS=D & EB & $46 \%$ & $7.00 \%$ \\
SR 416 & $\begin{array}{l}\text { From Intersection with U.S. 321 } \\
\text { to Intersection with Webb Creek }\end{array}$ & 220 & NB & $55 \%$ & $7.00 \%$ \\
& Rd & LOS=B & SB & $45 \%$ & \\
U.S. 321 & From Intersection with SR 416 & 1723 & EB & $38 \%$ & $5.00 \%$ \\
& to Outside of Gatlinburg & LOS=C & WB & $62 \%$ & \\
Foothill 8B & From Cosby to Pittman Center & 240 & EB & $55 \%$ & $0.00 \%$ \\
& & LOS=C & WB & $45 \%$ & \\
\hline
\end{tabular}

Table J.39. Weekend A.M. peak hour traffic projections for 2026-interchange at U.S. 321 (both options) $-8 B$ opened prior to completion of $8 \mathrm{C}$

\begin{tabular}{llrrrr}
\hline $\begin{array}{c}\text { Road section } \\
\text { name }\end{array}$ & \multicolumn{1}{c}{ Range } & $\begin{array}{c}\text { Traffic } \\
\text { volume }\end{array}$ & Directional split & $\begin{array}{c}\text { Percentage } \\
\text { of trucks }\end{array}$ \\
\hline U.S. 321 & $\begin{array}{l}\text { From Intersection with Foothills } \\
\text { Parkway Section 8A to } \\
\text { Convergence with SR 32 }\end{array}$ & 1746 & NB & $51 \%$ & $9.00 \%$ \\
& LOS=C & SB & $49 \%$ & \\
U.S. 321 & $\begin{array}{l}\text { From U.S. 321 Convergence } \\
\text { with SR 32 to Intersection with }\end{array}$ & 1703 & WB & $55 \%$ & $7.00 \%$ \\
& SR 416 & LOS=E & EB & $45 \%$ & \\
SR 416 & $\begin{array}{l}\text { From Intersection with U.S. 321 } \\
\text { to Intersection with Webb Creek }\end{array}$ & 231 & NB & $38 \%$ & $7.00 \%$ \\
& Rd & LOS=B & SB & $62 \%$ & \\
U.S. 321 & From Intersection with SR 416 & 2263 & EB & $36 \%$ & $5.00 \%$ \\
& to Outside of Gatlinburg & LOS=D & WB & $64 \%$ & \\
Foothill 8B & From Cosby to Pittman Center & 348 & EB & $60 \%$ & $0.00 \%$ \\
& & LOS=C & WB & $40 \%$ & \\
\hline
\end{tabular}


Table J.40. Weekend P.M. peak hour traffic projections for 2006-interchange at U.S. 321 (both options) $-8 B$ opened prior to completion of $8 \mathrm{C}$

\begin{tabular}{|c|c|c|c|c|c|}
\hline $\begin{array}{c}\text { Road section } \\
\text { name }\end{array}$ & Range & $\begin{array}{c}\text { Traffic } \\
\text { volume }\end{array}$ & \multicolumn{2}{|c|}{ Directional split } & $\begin{array}{l}\text { Percentage } \\
\text { of trucks }\end{array}$ \\
\hline U.S. 321 & $\begin{array}{l}\text { From Intersection with Foothills } \\
\text { Parkway Section } 8 \text { A to } \\
\text { Convergence with SR } 32\end{array}$ & $\begin{array}{r}1272 \\
\mathrm{LOS}=\mathrm{B}\end{array}$ & $\begin{array}{l}\text { NB } \\
\text { SB }\end{array}$ & $\begin{array}{l}55 \% \\
45 \%\end{array}$ & $9.00 \%$ \\
\hline U.S. 321 & $\begin{array}{l}\text { From U.S. } 321 \text { Convergence } \\
\text { with SR } 32 \text { to Intersection with } \\
\text { SR } 416\end{array}$ & $\begin{array}{r}1313 \\
\mathrm{LOS}=\mathrm{E}\end{array}$ & $\begin{array}{l}\text { WB } \\
\text { EB }\end{array}$ & $\begin{array}{l}54 \% \\
46 \%\end{array}$ & $7.00 \%$ \\
\hline SR 416 & $\begin{array}{l}\text { From Intersection with U.S. } 321 \\
\text { to Intersection with Webb Creek } \\
\text { Rd }\end{array}$ & $\begin{array}{r}269 \\
\mathrm{LOS}=\mathrm{B}\end{array}$ & $\begin{array}{l}\text { NB } \\
\text { SB }\end{array}$ & $\begin{array}{l}50 \% \\
50 \%\end{array}$ & $7.00 \%$ \\
\hline U.S. 321 & $\begin{array}{l}\text { From Intersection with SR } 416 \\
\text { to Outside of Gatlinburg }\end{array}$ & $\begin{array}{r}1711 \\
\mathrm{LOS}=\mathrm{C}\end{array}$ & $\begin{array}{l}\text { EB } \\
\text { WB }\end{array}$ & $\begin{array}{l}34 \% \\
66 \%\end{array}$ & $5.00 \%$ \\
\hline Foothill 8B & From Cosby to Pittman Center & $\begin{array}{r}263 \\
\mathrm{LOS}=\mathrm{C}\end{array}$ & $\begin{array}{l}\text { EB } \\
\text { WB }\end{array}$ & $\begin{array}{l}58 \% \\
42 \%\end{array}$ & $0.00 \%$ \\
\hline
\end{tabular}

Table J.41. Weekend P.M. peak hour traffic projections for 2026-interchange at U.S. 321 (both options) - $8 \mathrm{~B}$ opened prior to completion of $8 \mathrm{C}$

\begin{tabular}{|c|c|c|c|c|c|}
\hline $\begin{array}{c}\text { Road section } \\
\text { name }\end{array}$ & Range & $\begin{array}{l}\text { Traffic } \\
\text { volume }\end{array}$ & Dir & plit & $\begin{array}{l}\text { Percentage } \\
\text { of trucks }\end{array}$ \\
\hline U.S. 321 & $\begin{array}{l}\text { From Intersection with Foothills } \\
\text { Parkway Section } 8 \mathrm{~A} \text { to } \\
\text { Convergence with SR } 32\end{array}$ & $\begin{array}{r}1671 \\
\mathrm{LOS}=\mathrm{C}\end{array}$ & $\begin{array}{l}\text { NB } \\
\text { SB }\end{array}$ & $\begin{array}{l}54 \% \\
46 \%\end{array}$ & $9.00 \%$ \\
\hline U.S. 321 & $\begin{array}{l}\text { From U.S. } 321 \text { Convergence } \\
\text { with SR } 32 \text { to Intersection with } \\
\text { SR } 416\end{array}$ & $\begin{array}{r}1751 \\
\mathrm{LOS}=E\end{array}$ & $\begin{array}{l}\text { WB } \\
\text { EB }\end{array}$ & $\begin{array}{l}53 \% \\
47 \%\end{array}$ & $7.00 \%$ \\
\hline SR 416 & $\begin{array}{l}\text { From Intersection with U.S. } 321 \\
\text { to Intersection with Webb Creek } \\
\text { Rd }\end{array}$ & $\begin{array}{r}315 \\
\mathrm{LOS}=\mathrm{B}\end{array}$ & $\begin{array}{l}\text { NB } \\
\text { SB }\end{array}$ & $\begin{array}{l}37 \% \\
63 \%\end{array}$ & $7.00 \%$ \\
\hline U.S. 321 & $\begin{array}{l}\text { From Intersection with SR } 416 \\
\text { to Outside of Gatlinburg }\end{array}$ & $\begin{array}{r}2304 \\
\text { LOS }=D\end{array}$ & $\begin{array}{l}\text { EB } \\
\text { WB }\end{array}$ & $\begin{array}{l}33 \% \\
67 \%\end{array}$ & $5.00 \%$ \\
\hline Foothill 8B & From Cosby to Pittman Center & $\begin{array}{r}376 \\
\mathrm{LOS}=\mathrm{C}\end{array}$ & $\begin{array}{l}\text { EB } \\
\text { WB }\end{array}$ & $\begin{array}{l}64 \% \\
36 \%\end{array}$ & $0.00 \%$ \\
\hline
\end{tabular}


Table J.42. Weekday A.M. peak hour traffic projections for 2006-no-build alternative

\begin{tabular}{|c|c|c|c|c|c|}
\hline $\begin{array}{l}\text { Road section } \\
\text { name } \\
\end{array}$ & Range & $\begin{array}{c}\text { Traffic } \\
\text { volume } \\
\end{array}$ & \multicolumn{2}{|c|}{ Directional split } & $\begin{array}{c}\text { Percentage } \\
\text { of trucks }\end{array}$ \\
\hline U.S. 321 & $\begin{array}{l}\text { From Intersection with Foothills } \\
\text { Parkway Section } 8 \text { A to } \\
\text { Convergence with SR } 32\end{array}$ & $\begin{array}{r}1042 \\
L O S=B\end{array}$ & SB & $44 \%$ & $9.00 \%$ \\
\hline U.S. 321 & $\begin{array}{l}\text { From U.S. } 321 \text { Convergence } \\
\text { with SR } 32 \text { to Intersection with } \\
\text { SR } 416\end{array}$ & $\begin{array}{r}940 \\
\mathrm{LOS}=\mathrm{D}\end{array}$ & WB & $\begin{array}{l}55 \% \\
45 \%\end{array}$ & $7.00 \%$ \\
\hline SR 416 & $\begin{array}{l}\text { From Intersection with U.S. } 321 \\
\text { to Intersection with Webb Creek } \\
\text { Rd }\end{array}$ & $\begin{array}{r}214 \\
\mathrm{LOS}=\mathrm{B}\end{array}$ & NB & $\begin{array}{l}49 \% \\
51 \%\end{array}$ & $7.00 \%$ \\
\hline U.S. 321 & $\begin{array}{l}\text { From Intersection with SR } 416 \\
\text { to Outside of Gatlinburg }\end{array}$ & $\begin{array}{r}1334 \\
\mathrm{LOS}=\mathrm{B}\end{array}$ & $\begin{array}{l}\text { EB } \\
\text { WB }\end{array}$ & $\begin{array}{l}35 \% \\
65 \%\end{array}$ & $5.00 \%$ \\
\hline Foothill 8B & From Cosby to Pittman Center & 0 & $\begin{array}{l}\text { EB } \\
\text { WB }\end{array}$ & $\begin{array}{l}0 \% \\
0 \% \\
\end{array}$ & $0.00 \%$ \\
\hline
\end{tabular}

Table J.43. Weekday A.M. peak hour traffic projections for 2026-no-build alternative

\begin{tabular}{|c|c|c|c|c|c|}
\hline $\begin{array}{l}\text { Road section } \\
\text { name } \\
\end{array}$ & Range & $\begin{array}{l}\text { Traffic } \\
\text { volume } \\
\end{array}$ & Dir & plit & $\begin{array}{l}\text { Percentage } \\
\text { of trucks }\end{array}$ \\
\hline U.S. 321 & $\begin{array}{l}\text { From Intersection with Foothills } \\
\text { Parkway Section } 8 \text { A to } \\
\text { Convergence with SR } 32\end{array}$ & $\begin{array}{r}1212 \\
\mathrm{LOS}=\mathrm{B}\end{array}$ & $\begin{array}{l}\text { NB } \\
\text { SB }\end{array}$ & $\begin{array}{l}54 \% \\
46 \%\end{array}$ & $9.00 \%$ \\
\hline U.S. 321 & $\begin{array}{l}\text { From U.S. } 321 \text { Convergence } \\
\text { with SR } 32 \text { to Intersection with } \\
\text { SR } 416\end{array}$ & $\begin{array}{r}1051 \\
\mathrm{LOS}=\mathrm{D}\end{array}$ & $\begin{array}{l}\text { WB } \\
\text { EB }\end{array}$ & $\begin{array}{l}52 \% \\
48 \%\end{array}$ & $7.00 \%$ \\
\hline SR 416 & $\begin{array}{l}\text { From Intersection with U.S. } 321 \\
\text { to Intersection with Webb Creek } \\
\text { Rd }\end{array}$ & $\begin{array}{r}173 \\
\mathrm{LOS}=\mathrm{B}\end{array}$ & $\begin{array}{l}\text { NB } \\
\text { SB }\end{array}$ & $\begin{array}{l}37 \% \\
63 \%\end{array}$ & $7.00 \%$ \\
\hline U.S. 321 & $\begin{array}{l}\text { From Intersection with SR } 416 \\
\text { to Outside of Gatlinburg }\end{array}$ & $\begin{array}{r}1649 \\
\operatorname{LOS}=\mathrm{C}\end{array}$ & $\begin{array}{l}\text { EB } \\
\text { WB }\end{array}$ & $\begin{array}{l}29 \% \\
71 \%\end{array}$ & $5.00 \%$ \\
\hline Foothill 8B & From Cosby to Pittman Center & 0 & $\begin{array}{l}\text { EB } \\
\text { WB }\end{array}$ & $\begin{array}{l}0 \% \\
0 \% \\
\end{array}$ & $0.00 \%$ \\
\hline
\end{tabular}


Table J.44. Weekday P.M. peak hour traffic projections for 2006-no-build alternative

\begin{tabular}{|c|c|c|c|c|c|}
\hline $\begin{array}{c}\text { Road section } \\
\text { name }\end{array}$ & Range & $\begin{array}{c}\text { Traffic } \\
\text { volume }\end{array}$ & Dir & split & $\begin{array}{l}\text { Percentage } \\
\text { of trucks }\end{array}$ \\
\hline U.S. 321 & $\begin{array}{l}\text { From Intersection with Foothills } \\
\text { Parkway Section } 8 \text { A to } \\
\text { Convergence with SR } 32\end{array}$ & $\begin{array}{r}1106 \\
\mathrm{LOS}=\mathrm{B}\end{array}$ & SB & $\begin{array}{l}58 \% \\
42 \%\end{array}$ & $9.00 \%$ \\
\hline U.S. 321 & $\begin{array}{l}\text { From U.S. } 321 \text { Convergence } \\
\text { with SR } 32 \text { to Intersection with } \\
\text { SR } 416\end{array}$ & $\begin{array}{r}981 \\
\text { LOS }=D\end{array}$ & WB & $\begin{array}{l}52 \% \\
48 \%\end{array}$ & $7.00 \%$ \\
\hline SR 416 & $\begin{array}{l}\text { From Intersection with U.S. } 321 \\
\text { to Intersection with Webb Creek } \\
\text { Rd }\end{array}$ & $\begin{array}{r}204 \\
\mathrm{LOS}=\mathrm{B}\end{array}$ & NB & $\begin{array}{l}54 \% \\
46 \%\end{array}$ & $7.00 \%$ \\
\hline U.S. 321 & $\begin{array}{l}\text { From Intersection with SR } 416 \\
\text { to Outside of Gatlinburg }\end{array}$ & $\begin{array}{r}1164 \\
\operatorname{LOS}=B\end{array}$ & WB & $\begin{array}{l}32 \% \\
68 \%\end{array}$ & $5.00 \%$ \\
\hline Foothill 8B & From Cosby to Pittman Center & 0 & $\begin{array}{l}\text { EB } \\
\text { WB }\end{array}$ & $\begin{array}{l}0 \% \\
0 \%\end{array}$ & $0.00 \%$ \\
\hline
\end{tabular}

Table J.45. Weekday P.M. peak hour traffic projections for 2026-no-build alternative

\begin{tabular}{llrrrr}
\hline $\begin{array}{c}\text { Road section } \\
\text { name }\end{array}$ & \multicolumn{1}{c}{ Range } & $\begin{array}{c}\text { Traffic } \\
\text { volume }\end{array}$ & \multicolumn{1}{c}{ Directional split } & $\begin{array}{c}\text { Percentage } \\
\text { of trucks }\end{array}$ \\
\hline U.S. 321 & $\begin{array}{l}\text { From Intersection with Foothills } \\
\text { Parkway Section 8A to } \\
\text { Convergence with SR 32 }\end{array}$ & 1446 & NB & $58 \%$ & $9.00 \%$ \\
U.S. 321 & $\begin{array}{l}\text { From U.S. 321 Convergence } \\
\text { with SR 32 to Intersection with }\end{array}$ & 1290 & WB & $51 \%$ & $7.00 \%$ \\
& SR 416 & LOS=D & EB & $49 \%$ & \\
SR 416 & $\begin{array}{l}\text { From Intersection with U.S. 321 } \\
\text { to Intersection with Webb Creek }\end{array}$ & LOS=B & SB & $57 \%$ & \\
& Rd & NB & $43 \%$ & $7.00 \%$ \\
U.S. 321 & $\begin{array}{l}\text { From Intersection with SR 416 } \\
\text { to Outside of Gatlinburg }\end{array}$ & 1535 & EB & $30 \%$ & $5.00 \%$ \\
Foothill 8B & From Cosby to Pittman Center & LOS=B & WB & $70 \%$ & \\
& & & EB & $0 \%$ & $0.00 \%$ \\
\hline
\end{tabular}


Table J.46. Weekend A.M. peak hour traffic projections for 2006-no-build alternative

\begin{tabular}{|c|c|c|c|c|c|}
\hline $\begin{array}{l}\text { Road section } \\
\text { name }\end{array}$ & Range & $\begin{array}{c}\text { Traffic } \\
\text { volume }\end{array}$ & & split & $\begin{array}{c}\text { Percentage } \\
\text { of trucks }\end{array}$ \\
\hline U.S. 321 & $\begin{array}{l}\text { From Intersection with Foothills } \\
\text { Parkway Section } 8 \text { A to } \\
\text { Convergence with SR } 32\end{array}$ & $\begin{array}{r}1458 \\
\mathrm{LOS}=\mathrm{B}\end{array}$ & SB & $49 \%$ & $9.00 \%$ \\
\hline U.S. 321 & $\begin{array}{l}\text { From U.S. } 321 \text { Convergence } \\
\text { with SR } 32 \text { to Intersection with } \\
\text { SR } 416\end{array}$ & $\begin{array}{r}1291 \\
\mathrm{LOS}=\mathrm{E}\end{array}$ & $\begin{array}{l}\text { WB } \\
\text { EB }\end{array}$ & $\begin{array}{l}56 \% \\
44 \%\end{array}$ & $7.00 \%$ \\
\hline SR 416 & $\begin{array}{l}\text { From Intersection with U.S. } 321 \\
\text { to Intersection with Webb Creek } \\
\mathrm{Rd}\end{array}$ & $\begin{array}{r}220 \\
\mathrm{LOS}=\mathrm{B}\end{array}$ & NB & $\begin{array}{l}55 \% \\
45 \%\end{array}$ & $7.00 \%$ \\
\hline U.S. 321 & $\begin{array}{l}\text { From Intersection with SR } 416 \\
\text { to Outside of Gatlinburg }\end{array}$ & $\begin{array}{r}1635 \\
\mathrm{LOS}=\mathrm{C}\end{array}$ & $\mathrm{EB}$ & $\begin{array}{l}37 \% \\
63 \%\end{array}$ & $5.00 \%$ \\
\hline Foothill 8B & From Cosby to Pittman Center & 0 & $\begin{array}{l}\text { EB } \\
\text { WB }\end{array}$ & $\begin{array}{l}0 \% \\
0 \%\end{array}$ & $0.00 \%$ \\
\hline
\end{tabular}

Table J.47. Weekend A.M. peak hour traffic projections for 2026-no-build alternative

\begin{tabular}{|c|c|c|c|c|c|}
\hline $\begin{array}{c}\text { Road section } \\
\text { name }\end{array}$ & Range & $\begin{array}{c}\text { Traffic } \\
\text { volume }\end{array}$ & & split & $\begin{array}{c}\text { Percentage } \\
\text { of trucks }\end{array}$ \\
\hline U.S. 321 & $\begin{array}{l}\text { From Intersection with Foothills } \\
\text { Parkway Section } 8 \text { A to } \\
\text { Convergence with SR } 32\end{array}$ & $\begin{array}{r}1905 \\
\mathrm{LOS}=\mathrm{C}\end{array}$ & NB & $\begin{array}{l}51 \% \\
49 \%\end{array}$ & $9.00 \%$ \\
\hline U.S. 321 & $\begin{array}{l}\text { From U.S. } 321 \text { Convergence } \\
\text { with SR } 32 \text { to Intersection with } \\
\text { SR } 416\end{array}$ & $\begin{array}{r}1722 \\
\mathrm{LOS}=\mathrm{E}\end{array}$ & $\begin{array}{l}\text { WB } \\
\text { EB }\end{array}$ & $\begin{array}{l}56 \% \\
44 \%\end{array}$ & $7.00 \%$ \\
\hline SR 416 & $\begin{array}{l}\text { From Intersection with U.S. } 321 \\
\text { to Intersection with Webb Creek } \\
\text { Rd }\end{array}$ & $\begin{array}{r}231 \\
\mathrm{LOS}=\mathrm{B}\end{array}$ & NB & $\begin{array}{l}38 \% \\
62 \%\end{array}$ & $7.00 \%$ \\
\hline U.S. 321 & $\begin{array}{l}\text { From Intersection with SR } 416 \\
\text { to Outside of Gatlinburg }\end{array}$ & $\begin{array}{r}2108 \\
\text { LOS }=D\end{array}$ & WB & $\begin{array}{l}33 \% \\
67 \%\end{array}$ & $5.00 \%$ \\
\hline Foothill 8B & From Cosby to Pittman Center & 0 & $\begin{array}{l}\text { EB } \\
\text { WB }\end{array}$ & $\begin{array}{l}0 \% \\
0 \%\end{array}$ & $0.00 \%$ \\
\hline
\end{tabular}


Table J.48. Weekend P.M. peak hour traffic projections for 2006-no-build alternative

\begin{tabular}{llrrrr}
\hline $\begin{array}{c}\text { Road section } \\
\text { name }\end{array}$ & \multicolumn{1}{c}{ Range } & $\begin{array}{c}\text { Traffic } \\
\text { volume }\end{array}$ & Directional split & $\begin{array}{c}\text { Percentage } \\
\text { of trucks }\end{array}$ \\
\hline U.S. 321 & $\begin{array}{l}\text { From Intersection with Foothills } \\
\text { Parkway Section 8A to } \\
\text { Convergence with SR 32 }\end{array}$ & 1404 & NB & $54 \%$ & $9.00 \%$ \\
U.S. 321 & $\begin{array}{l}\text { From U.S. 321 Convergence } \\
\text { with SR 32 to Intersection with }\end{array}$ & 1344 & WB & $55 \%$ & $7.00 \%$ \\
& SR 416 & LOS=E & EB & $45 \%$ & \\
SR 416 & $\begin{array}{l}\text { From Intersection with U.S. 321 } \\
\text { to Intersection with Webb Creek }\end{array}$ & 269 & NB & $50 \%$ & $7.00 \%$ \\
& Rd & LOS=B & SB & $50 \%$ & \\
U.S. 321 & From Intersection with SR 416 & 1622 & EB & $32 \%$ & $5.00 \%$ \\
& to Outside of Gatlinburg & LOS=C & WB & $68 \%$ & \\
Foothill 8B & From Cosby to Pittman Center & 0 & EB & $0 \%$ & $0.00 \%$ \\
& & & WB & $0 \%$ & \\
\hline
\end{tabular}

Table J.49. Weekend P.M. peak hour traffic projections for 2026-no-build alternative

\begin{tabular}{|c|c|c|c|c|c|}
\hline $\begin{array}{l}\text { Road section } \\
\text { name }\end{array}$ & Range & $\begin{array}{l}\text { Traffic } \\
\text { volume } \\
\end{array}$ & Dir & split & $\begin{array}{c}\text { Percentage } \\
\text { of trucks }\end{array}$ \\
\hline U.S. 321 & $\begin{array}{l}\text { From Intersection with Foothills } \\
\text { Parkway Section } 8 \text { A to } \\
\text { Convergence with SR } 32\end{array}$ & $\begin{array}{r}1837 \\
\text { LOS }=\mathrm{C}\end{array}$ & $\begin{array}{l}\text { NB } \\
\text { SB }\end{array}$ & $\begin{array}{l}54 \% \\
46 \%\end{array}$ & $9.00 \%$ \\
\hline U.S. 321 & $\begin{array}{l}\text { From U.S. } 321 \text { Convergence } \\
\text { with SR } 32 \text { to Intersection with } \\
\text { SR } 416\end{array}$ & $\begin{array}{r}1771 \\
\mathrm{LOS}=\mathrm{E}\end{array}$ & $\begin{array}{l}\text { WB } \\
\text { EB }\end{array}$ & $\begin{array}{l}54 \% \\
46 \%\end{array}$ & $7.00 \%$ \\
\hline SR 416 & $\begin{array}{l}\text { From Intersection with U.S. } 321 \\
\text { to Intersection with Webb Creek } \\
\text { Rd }\end{array}$ & $\begin{array}{r}315 \\
\mathrm{LOS}=\mathrm{B}\end{array}$ & $\begin{array}{l}\text { NB } \\
\text { SB }\end{array}$ & $\begin{array}{l}37 \% \\
63 \%\end{array}$ & $7.00 \%$ \\
\hline U.S. 321 & $\begin{array}{l}\text { From Intersection with SR } 416 \\
\text { to Outside of Gatlinburg }\end{array}$ & $\begin{array}{r}2150 \\
\text { LOS }=D\end{array}$ & $\begin{array}{l}\text { EB } \\
\text { WB }\end{array}$ & $\begin{array}{l}30 \% \\
70 \%\end{array}$ & $5.00 \%$ \\
\hline Foothill 8B & From Cosby to Pittman Center & 0 & $\begin{array}{l}\text { EB } \\
\text { WB }\end{array}$ & $\begin{array}{l}0 \% \\
0 \%\end{array}$ & $0.00 \%$ \\
\hline
\end{tabular}




\title{
Appendix K
}

\section{INTERSECTION TRAFFIC VOLUME RESULTS FOR THE FIVE BUILD OPTIONS}

\author{
S. M. Chin
}

Oak Ridge National Laboratory

Oak Ridge, Tennessee

R. Gibson

Oak Ridge National Laboratory

Oak Ridge, Tennessee

August 1995 


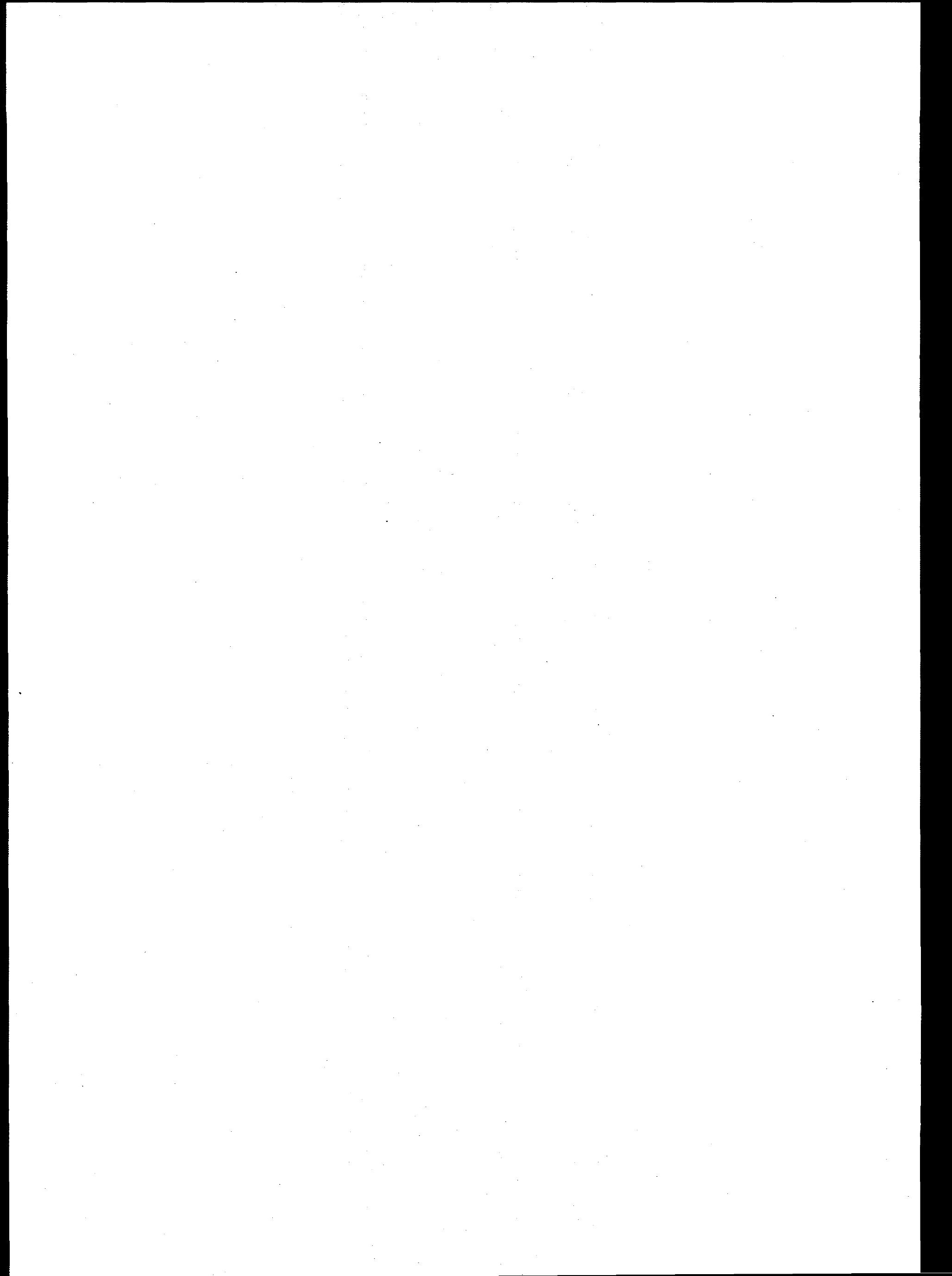


Table K.1. Weekday A.M. peak-hour intersection volume projections for 2006-construct 8B with no interchanges option

\begin{tabular}{|c|c|c|c|c|c|}
\hline \multirow[b]{2}{*}{ Intersection location } & \multirow[b]{2}{*}{ Approach } & \multirow{2}{*}{$\begin{array}{l}\text { Total } \\
\text { vehicles } \\
\text { per hour }\end{array}$} & \multicolumn{3}{|c|}{ Directional information } \\
\hline & & & $\begin{array}{c}\text { Turning } \\
\text { movement }\end{array}$ & Percent & Counts \\
\hline U.S. 321 Intersection w/ Foothills Pkwy & FH Pkwy 8A WB & 222 & $\begin{array}{l}\text { Right Tum } \\
\text { Through } \\
\text { Left Tum }\end{array}$ & $\begin{array}{r}13.06 \% \\
7.66 \% \\
79.28 \%\end{array}$ & $\begin{array}{r}29 \\
17 \\
176\end{array}$ \\
\hline & FH Pkwy 8B EB & 97 & $\begin{array}{l}\text { Right Turn } \\
\text { Through } \\
\text { Left Tum }\end{array}$ & $\begin{array}{r}6.19 \% \\
50.52 \% \\
43.30 \%\end{array}$ & $\begin{array}{r}6 \\
49 \\
42\end{array}$ \\
\hline & U.S. $321 \mathrm{SB}$ & 537 & $\begin{array}{l}\text { Right Turn } \\
\text { Through } \\
\text { Left Turn }\end{array}$ & $\begin{array}{r}12.66 \% \\
77.47 \% \\
9.87 \%\end{array}$ & $\begin{array}{r}68 \\
416 \\
53\end{array}$ \\
\hline & U.S. $321 \mathrm{NB}$ & 660 & $\begin{array}{l}\text { Right Tum } \\
\text { Through } \\
\text { Left Tum }\end{array}$ & $\begin{array}{r}21.67 \% \\
77.73 \% \\
0.61 \%\end{array}$ & $\begin{array}{r}143 \\
513 \\
4\end{array}$ \\
\hline U.S. 321 Convergence w/ SR 32 & U.S. $321 \mathrm{~EB}$ & 416 & $\begin{array}{l}\text { Right Tum } \\
\text { Left Tum }\end{array}$ & $\begin{array}{l}12.74 \% \\
87.26 \%\end{array}$ & $\begin{array}{r}53 \\
363\end{array}$ \\
\hline & U.S. 321 SB & 228 & $\begin{array}{l}\text { Right Turn } \\
\text { Through }\end{array}$ & $\begin{array}{l}71.05 \% \\
28.95 \%\end{array}$ & $\begin{array}{r}162 \\
66\end{array}$ \\
\hline & SR $32 \mathrm{NB}$ & 76 & $\begin{array}{l}\text { Through } \\
\text { Left Turn }\end{array}$ & $\begin{array}{l}71.05 \% \\
28.95 \%\end{array}$ & $\begin{array}{l}54 \\
22\end{array}$ \\
\hline U.S. 321 Intersection w/ SR 416 & SR 416 SB & 126 & $\begin{array}{l}\text { Right Tum } \\
\text { Left Turn }\end{array}$ & $\begin{array}{l}76.19 \% \\
23.81 \%\end{array}$ & $\begin{array}{l}96 \\
30\end{array}$ \\
\hline & U.S. 321 WB & 675 & $\begin{array}{l}\text { Right Turn } \\
\text { Through }\end{array}$ & $\begin{array}{r}5.04 \% \\
94.96 \%\end{array}$ & $\begin{array}{r}34 \\
641\end{array}$ \\
\hline & U.S. $321 \mathrm{~EB}$ & 416 & $\begin{array}{l}\text { Through } \\
\text { Left Turn }\end{array}$ & $\begin{array}{l}73.56 \% \\
26.44 \%\end{array}$ & $\begin{array}{l}306 \\
110\end{array}$ \\
\hline SR 416 Intersection w/ Webb Creek Rd & Webb CrRd WB & 54 & $\begin{array}{l}\text { Right Turn } \\
\text { Left Turn }\end{array}$ & $\begin{array}{r}3.70 \% \\
96.30 \%\end{array}$ & $\begin{array}{r}2 \\
52\end{array}$ \\
\hline & SR 416 SB & 42 & $\begin{array}{l}\text { Through } \\
\text { Left Turn }\end{array}$ & $\begin{array}{r}97.62 \% \\
2.38 \%\end{array}$ & $\begin{array}{r}41 \\
1\end{array}$ \\
\hline & SR $416 \mathrm{NB}$ & 65 & $\begin{array}{l}\text { Right Tumn } \\
\text { Through }\end{array}$ & $\begin{array}{l}49.23 \% \\
50.77 \%\end{array}$ & $\begin{array}{l}32 \\
33\end{array}$ \\
\hline SR 416 Intersection w/ Foothills Pkwy 8B/8C & FH Pkwy 8B WB & 83 & $\begin{array}{l}\text { Right Turn } \\
\text { Through } \\
\text { Left Turn }\end{array}$ & $\begin{array}{r}0.00 \% \\
100.00 \% \\
0.00 \%\end{array}$ & $\begin{array}{r}0 \\
83 \\
0\end{array}$ \\
\hline & FH Pkwy 8C EB & 96 & $\begin{array}{l}\text { Right Turn } \\
\text { Through } \\
\text { Left Turn }\end{array}$ & $\begin{array}{r}0.00 \% \\
100.00 \% \\
0.00 \%\end{array}$ & $\begin{array}{r}0 \\
96 \\
0\end{array}$ \\
\hline & SR 416 SB & 93 & $\begin{array}{l}\text { Right Turn } \\
\text { Through } \\
\text { Left Turn }\end{array}$ & $\begin{array}{r}0.00 \% \\
100.00 \% \\
0.00 \%\end{array}$ & $\begin{array}{r}0 \\
93 \\
0\end{array}$ \\
\hline & SR $416 \mathrm{NB}$ & 65 & $\begin{array}{l}\text { Right Turn } \\
\text { Through } \\
\text { Left Tum }\end{array}$ & $\begin{array}{r}0.00 \% \\
100.00 \% \\
0.00 \%\end{array}$ & $\begin{array}{r}0 \\
65 \\
0\end{array}$ \\
\hline
\end{tabular}


Table K.2. Weekday P.M. peak-hour intersection volume projections for 2006-construct 8B with no interchanges option

\begin{tabular}{|c|c|c|c|c|c|}
\hline \multirow[b]{2}{*}{ Intersection location } & \multirow[b]{2}{*}{ Approach } & \multirow{2}{*}{$\begin{array}{c}\text { Total } \\
\text { vehicles } \\
\text { per hour }\end{array}$} & \multicolumn{3}{|c|}{ Directional information } \\
\hline & & & $\begin{array}{l}\text { Turning } \\
\text { movement }\end{array}$ & Percent & Counts \\
\hline \multirow[t]{4}{*}{ U.S. 321 Intersection w/ Foothills Pkwy } & FH Pkwy 8A WB & 163 & $\begin{array}{l}\text { Right Turn } \\
\text { Through } \\
\text { Left Turn }\end{array}$ & $\begin{array}{r}15.95 \% \\
7.36 \% \\
76.69 \%\end{array}$ & $\begin{array}{r}26 \\
12 \\
125\end{array}$ \\
\hline & FH Pkwy 8B EB & 128 & $\begin{array}{l}\text { Right Turn } \\
\text { Through } \\
\text { Left Turn }\end{array}$ & $\begin{array}{r}6.25 \% \\
50.78 \% \\
42.97 \%\end{array}$ & $\begin{array}{r}8 \\
65 \\
55\end{array}$ \\
\hline & U.S. $321 \mathrm{SB}$ & 555 & $\begin{array}{l}\text { Right Turn } \\
\text { Through } \\
\text { Left Turn }\end{array}$ & $\begin{array}{r}13.69 \% \\
83.24 \% \\
3.06 \%\end{array}$ & $\begin{array}{r}76 \\
462 \\
17\end{array}$ \\
\hline & U.S. $321 \mathrm{NB}$ & 671 & $\begin{array}{l}\text { Right Turn } \\
\text { Through } \\
\text { Left Turn }\end{array}$ & $\begin{array}{r}14.31 \% \\
84.95 \% \\
0.75 \%\end{array}$ & $\begin{array}{r}96 \\
570 \\
5\end{array}$ \\
\hline \multirow[t]{3}{*}{ U.S. 321 Convergence w/ SR 32} & U.S. $321 \mathrm{~EB}$ & 551 & $\begin{array}{l}\text { Right Tum } \\
\text { Left Turn }\end{array}$ & $\begin{array}{l}13.07 \% \\
86.93 \%\end{array}$ & $\begin{array}{r}72 \\
479\end{array}$ \\
\hline & U.S. $321 \mathrm{SB}$ & 228 & $\begin{array}{l}\text { Right Turn } \\
\text { Through }\end{array}$ & $\begin{array}{l}71.49 \% \\
28.51 \%\end{array}$ & $\begin{array}{r}163 \\
65\end{array}$ \\
\hline & SR $32 \mathrm{NB}$ & 54 & $\begin{array}{l}\text { Through } \\
\text { Left Turn }\end{array}$ & $\begin{array}{l}68.52 \% \\
31.48 \%\end{array}$ & $\begin{array}{l}37 \\
17\end{array}$ \\
\hline \multirow[t]{3}{*}{ U.S. 321 Intersection w/ SR 416} & SR 416 SB & 82 & $\begin{array}{l}\text { Right Tum } \\
\text { Left Tum }\end{array}$ & $\begin{array}{l}79.27 \% \\
20.73 \%\end{array}$ & $\begin{array}{l}65 \\
17\end{array}$ \\
\hline & U.S. 321 WB & 680 & $\begin{array}{l}\text { Right Turn } \\
\text { Through }\end{array}$ & $\begin{array}{l}10.88 \% \\
89.12 \%\end{array}$ & $\begin{array}{r}74 \\
606\end{array}$ \\
\hline & U.S. 321 EB & 325 & $\begin{array}{l}\text { Through } \\
\text { Left Tum }\end{array}$ & $\begin{array}{l}81.54 \% \\
18.46 \%\end{array}$ & $\begin{array}{r}265 \\
60\end{array}$ \\
\hline \multirow[t]{3}{*}{ SR 416 Intersection webb Creek Rd } & Webb Cr Rd WB & 58 & $\begin{array}{l}\text { Right Tum } \\
\text { Left Turn }\end{array}$ & $\begin{array}{r}5.17 \% \\
94.83 \%\end{array}$ & $\begin{array}{r}3 \\
55\end{array}$ \\
\hline & SR 416 SB & 53 & $\begin{array}{l}\text { Through } \\
\text { Left Turn }\end{array}$ & $\begin{array}{r}98.11 \% \\
1.89 \%\end{array}$ & $\begin{array}{r}52 \\
1\end{array}$ \\
\hline & SR $416 \mathrm{NB}$ & 84 & $\begin{array}{l}\text { Right Turn } \\
\text { Through }\end{array}$ & $\begin{array}{l}45.24 \% \\
54.76 \%\end{array}$ & $\begin{array}{l}38 \\
46\end{array}$ \\
\hline \multirow[t]{4}{*}{ SR 416 Intersection w/ Foothills Pkwy 8B/8C } & FH Pkwy 8B WB & 84 & $\begin{array}{l}\text { Right Turn } \\
\text { Through } \\
\text { Left Turn }\end{array}$ & $\begin{array}{r}0.00 \% \\
100.00 \% \\
0.00 \%\end{array}$ & $\begin{array}{r}0 \\
84 \\
0\end{array}$ \\
\hline & FH Pkwy 8C EB & 126 & $\begin{array}{l}\text { Right Tum } \\
\text { Through } \\
\text { Left Turn }\end{array}$ & $\begin{array}{r}0.00 \% \\
100.00 \% \\
0.00 \%\end{array}$ & $\begin{array}{r}0 \\
126 \\
0\end{array}$ \\
\hline & SR $416 \mathrm{SB}$ & 107 & $\begin{array}{l}\text { Right Turn } \\
\text { Through } \\
\text { Left Turn }\end{array}$ & $\begin{array}{r}0.00 \% \\
100.00 \% \\
0.00 \%\end{array}$ & $\begin{array}{r}0 \\
107 \\
0\end{array}$ \\
\hline & SR $416 \mathrm{NB}$ & 84 & $\begin{array}{l}\text { Right Turn } \\
\text { Through } \\
\text { Left Turn }\end{array}$ & $\begin{array}{r}0.00 \% \\
100.00 \% \\
0.00 \%\end{array}$ & $\begin{array}{r}0 \\
84 \\
0\end{array}$ \\
\hline
\end{tabular}


Table K.3. Weekend A.M. peak-hour intersection volume projections for 2006 -construct $8 B$ with no interchanges option

\begin{tabular}{|c|c|c|c|c|c|}
\hline \multirow[b]{2}{*}{ Intersection location } & \multirow[b]{2}{*}{ Approach } & \multirow{2}{*}{$\begin{array}{c}\text { Total } \\
\text { vehicles } \\
\text { per hour }\end{array}$} & \multicolumn{3}{|c|}{ Directional information } \\
\hline & & & $\begin{array}{c}\text { Turning } \\
\text { movement }\end{array}$ & Percent & Counts \\
\hline U.S. 321 Intersection w/ Foothills Pkwy & FH Pkwy 8A WB & 375 & $\begin{array}{l}\text { Right Tum } \\
\text { Through } \\
\text { Left Turn }\end{array}$ & $\begin{array}{r}13.07 \% \\
7.73 \% \\
79.20 \%\end{array}$ & $\begin{array}{r}49 \\
29 \\
297\end{array}$ \\
\hline & FH Pkwy 8B EB & 132 & $\begin{array}{l}\text { Right Tum } \\
\text { Through } \\
\text { Left Turn }\end{array}$ & $\begin{array}{r}6.06 \% \\
50.76 \% \\
43.18 \%\end{array}$ & $\begin{array}{r}8 \\
67 \\
57\end{array}$ \\
\hline & U.S. $321 \mathrm{SB}$ & 591 & $\begin{array}{l}\text { Right Tum } \\
\text { Through } \\
\text { Left Turn }\end{array}$ & $\begin{array}{r}12.69 \% \\
77.33 \% \\
9.98 \%\end{array}$ & $\begin{array}{r}75 \\
457 \\
59\end{array}$ \\
\hline & U.S. $321 \mathrm{NB}$ & 813 & $\begin{array}{l}\text { Right Tum } \\
\text { Through } \\
\text { Left Turn }\end{array}$ & $\begin{array}{r}21.77 \% \\
77.61 \% \\
0.62 \%\end{array}$ & $\begin{array}{r}177 \\
631 \\
5\end{array}$ \\
\hline U.S. 321 Convergence w/ SR 32 & U.S. $321 \mathrm{~EB}$ & 570 & $\begin{array}{l}\text { Right Tum } \\
\text { Left Turn }\end{array}$ & $\begin{array}{l}12.81 \% \\
87.19 \%\end{array}$ & $\begin{array}{r}73 \\
497\end{array}$ \\
\hline & U.S. $321 \mathrm{SB}$ & 519 & $\begin{array}{l}\text { Right Turn } \\
\text { Through }\end{array}$ & $\begin{array}{l}70.91 \% \\
29.09 \%\end{array}$ & $\begin{array}{l}368 \\
151\end{array}$ \\
\hline & SR $32 \mathrm{NB}$ & 104 & $\begin{array}{l}\text { Through } \\
\text { Left Turn }\end{array}$ & $\begin{array}{l}72.12 \% \\
27.88 \%\end{array}$ & $\begin{array}{l}75 \\
29\end{array}$ \\
\hline U.S. 321 Intersection w/ SR 416 & SR 416 SB & 137 & $\begin{array}{l}\text { Right Tum } \\
\text { Left Tum }\end{array}$ & $\begin{array}{l}76.64 \% \\
23.36 \%\end{array}$ & $\begin{array}{r}105 \\
32\end{array}$ \\
\hline & U.S. $321 \mathrm{WB}$ & 821 & $\begin{array}{l}\text { Right Turn } \\
\text { Through }\end{array}$ & $\begin{array}{r}5.24 \% \\
94.76 \%\end{array}$ & $\begin{array}{r}43 \\
778\end{array}$ \\
\hline & U.S. $321 \mathrm{~EB}$ & 530 & $\begin{array}{l}\text { Through } \\
\text { Left Turn }\end{array}$ & $\begin{array}{l}73.21 \% \\
26.79 \%\end{array}$ & $\begin{array}{l}388 \\
142\end{array}$ \\
\hline SR 416 Intersection w/ Webb Creek Rd & Webb Cr Rd WB & 34 & $\begin{array}{l}\text { Right Tum } \\
\text { Left Tum }\end{array}$ & $\begin{array}{r}2.94 \% \\
97.06 \%\end{array}$ & $\begin{array}{r}1 \\
33\end{array}$ \\
\hline . & SR 416 SB & 31 & $\begin{array}{l}\text { Through } \\
\text { Left Turn }\end{array}$ & $\begin{array}{r}96.77 \% \\
3.23 \%\end{array}$ & $\begin{array}{r}30 \\
1\end{array}$ \\
\hline & SR $416 \mathrm{NB}$ & 54 & $\begin{array}{l}\text { Right Turn } \\
\text { Through }\end{array}$ & $\begin{array}{l}48.15 \% \\
51.85 \%\end{array}$ & $\begin{array}{l}26 \\
28\end{array}$ \\
\hline SR 416 Intersection w/ Foothills Pkwy 8B/8C & FH Pkwy 8B WB & 108 & $\begin{array}{l}\text { Right Turn } \\
\text { Through } \\
\text { Left Turn }\end{array}$ & $\begin{array}{r}0.00 \% \\
100.00 \% \\
0.00 \%\end{array}$ & 108 \\
\hline & FH Pkwy 8C EB & 131 & $\begin{array}{l}\text { Right Turn } \\
\text { Through } \\
\text { Left Turn }\end{array}$ & $\begin{array}{r}0.00 \% \\
100.00 \% \\
0.00 \%\end{array}$ & 131 \\
\hline . & SR $416 \mathrm{SB}$ & 63 & $\begin{array}{l}\text { Right Turn } \\
\text { Through } \\
\text { Left Tum }\end{array}$ & $\begin{array}{r}0.00 \% \\
100.00 \% \\
0.00 \%\end{array}$ & 63 \\
\hline & SR $416 \mathrm{NB}$ & 54 & $\begin{array}{l}\text { Right Turn } \\
\text { Through } \\
\text { Left Turn }\end{array}$ & $\begin{array}{r}0.00 \% \\
100.00 \% \\
0.00 \%\end{array}$ & 54 \\
\hline
\end{tabular}


Table K.4. Weekend P.M. peak-hour intersection volume projections for 2006 -construct 8B with no interchanges option

\begin{tabular}{|c|c|c|c|c|c|}
\hline \multirow[b]{2}{*}{ Intersection location } & \multirow[b]{2}{*}{ Approach } & \multirow{2}{*}{$\begin{array}{c}\text { Total } \\
\text { vehicles } \\
\text { per hour }\end{array}$} & \multicolumn{3}{|c|}{ Directional information } \\
\hline & & & $\begin{array}{c}\text { Turning } \\
\text { movement }\end{array}$ & Percent & Counts \\
\hline \multirow[t]{4}{*}{ U.S. 321 Intersection w/ Foothills Pkwy } & FH Pkwy 8A WB & 229 & $\begin{array}{l}\text { Right Turn } \\
\text { Through } \\
\text { Left Turn }\end{array}$ & $\begin{array}{r}16.59 \% \\
7.42 \% \\
75.98 \%\end{array}$ & $\begin{array}{r}38 \\
17 \\
174\end{array}$ \\
\hline & FH Pkwy 8B EB & 153 & $\begin{array}{l}\text { Right Tum } \\
\text { Through } \\
\text { Left Tum }\end{array}$ & $\begin{array}{r}5.88 \% \\
50.98 \% \\
43.14 \%\end{array}$ & $\begin{array}{r}9 \\
78 \\
66\end{array}$ \\
\hline & U.S. $321 \mathrm{SB}$ & 651 & $\begin{array}{l}\text { Right Turn } \\
\text { Through } \\
\text { Left Tum }\end{array}$ & $\begin{array}{r}13.67 \% \\
83.26 \% \\
3.07 \%\end{array}$ & $\begin{array}{r}89 \\
542 \\
20\end{array}$ \\
\hline & U.S. $321 \mathrm{NB}$ & 766 & $\begin{array}{l}\text { Right Turn } \\
\text { Through } \\
\text { Left Turn }\end{array}$ & $\begin{array}{r}14.23 \% \\
85.12 \% \\
0.65 \%\end{array}$ & $\begin{array}{r}109 \\
652 \\
5\end{array}$ \\
\hline \multirow[t]{3}{*}{ U.S. 321 Convergence w/ SR 32} & U.S. $321 \mathrm{~EB}$ & 663 & $\begin{array}{l}\text { Right Turn } \\
\text { Left Turn }\end{array}$ & $\begin{array}{l}13.12 \% \\
86.88 \%\end{array}$ & $\begin{array}{r}87 \\
576\end{array}$ \\
\hline & U.S. 321 SB & 432 & $\begin{array}{l}\text { Right Turn } \\
\text { Through }\end{array}$ & $\begin{array}{l}71.30 \% \\
28.70 \%\end{array}$ & $\begin{array}{l}308 \\
124\end{array}$ \\
\hline & SR 32 NB & 69 & $\begin{array}{l}\text { Through } \\
\text { Left Turn }\end{array}$ & $\begin{array}{l}68.12 \% \\
31.88 \%\end{array}$ & $\begin{array}{l}47 \\
22\end{array}$ \\
\hline \multirow[t]{3}{*}{ U.S. 321 Intersection w/ SR 416} & SR 416 SB & 160 & $\begin{array}{l}\text { Right Tum } \\
\text { Left Tum }\end{array}$ & $\begin{array}{l}78.75 \% \\
21.25 \%\end{array}$ & $\begin{array}{r}126 \\
34\end{array}$ \\
\hline & U.S. $321 \mathrm{WB}$ & 908 & $\begin{array}{l}\text { Right Turn } \\
\text { Through }\end{array}$ & $\begin{array}{l}10.79 \% \\
89.21 \%\end{array}$ & $\begin{array}{r}98 \\
810\end{array}$ \\
\hline & U.S. 321 EB & 461 & $\begin{array}{l}\text { Through } \\
\text { Left Turn }\end{array}$ & $\begin{array}{l}81.78 \% \\
18.22 \%\end{array}$ & $\begin{array}{r}377 \\
84\end{array}$ \\
\hline \multirow[t]{3}{*}{ SR 416 Intersection w/ Webb Creek Rd } & Webb Cr Rd WB & 43 & $\begin{array}{l}\text { Right Turn } \\
\text { Left Turn }\end{array}$ & $\begin{array}{r}4.65 \% \\
95.35 \%\end{array}$ & $\begin{array}{r}2 \\
41\end{array}$ \\
\hline & SR 416 SB & 72 & $\begin{array}{l}\text { Through } \\
\text { Left Turn }\end{array}$ & $\begin{array}{r}98.61 \% \\
1.39 \%\end{array}$ & $\begin{array}{r}71 \\
1\end{array}$ \\
\hline & SR $416 \mathrm{NB}$ & 84 & $\begin{array}{l}\text { Right Turn } \\
\text { Through }\end{array}$ & $\begin{array}{l}45.24 \% \\
54.76 \%\end{array}$ & $\begin{array}{l}38 \\
46\end{array}$ \\
\hline \multirow[t]{4}{*}{ SR 416 Intersection w/ Foothills Pkwy 8B/8C } & FH Pkwy 8B WB & 101 & $\begin{array}{l}\text { Right Turn } \\
\text { Through } \\
\text { Left Tum }\end{array}$ & $\begin{array}{r}0.00 \% \\
100.00 \% \\
0.00 \%\end{array}$ & $\begin{array}{r}0 \\
101 \\
0\end{array}$ \\
\hline & FH Pkwy 8C EB & 152 & $\begin{array}{l}\text { Right Tum } \\
\text { Through } \\
\text { Left Tum }\end{array}$ & $\begin{array}{r}0.00 \% \\
100.00 \% \\
0.00 \%\end{array}$ & $\begin{array}{r}0 \\
152 \\
0\end{array}$ \\
\hline & SR 416 SB & 112 & $\begin{array}{l}\text { Right Turn } \\
\text { Through } \\
\text { Left Tum }\end{array}$ & $\begin{array}{r}0.00 \% \\
100.00 \% \\
0.00 \%\end{array}$ & $\begin{array}{r}0 \\
112 \\
0\end{array}$ \\
\hline & SR $416 \mathrm{NB}$ & 84 & $\begin{array}{l}\text { Right Turn } \\
\text { Through } \\
\text { Left Tum }\end{array}$ & $\begin{array}{r}0.00 \% \\
100.00 \% \\
0.00 \%\end{array}$ & $\begin{array}{r}0 \\
84 \\
0\end{array}$ \\
\hline
\end{tabular}


Table K.5. Weekday A.M. peak-hour intersection volume projections for 2026-construct 8B with no interchanges option

\begin{tabular}{|c|c|c|c|c|c|}
\hline \multirow[b]{2}{*}{ Intersection location } & \multirow[b]{2}{*}{ Approach } & \multirow{2}{*}{$\begin{array}{c}\text { Total } \\
\text { vehicles } \\
\text { per hour }\end{array}$} & \multicolumn{3}{|c|}{ Directional information } \\
\hline & & & $\begin{array}{c}\text { Turning } \\
\text { movement }\end{array}$ & Percent & Counts \\
\hline \multirow[t]{12}{*}{ U.S. 321 Intersection w/ Foothills Pkwy } & FH Pkwy 8A WB & 300 & Right Turn & $12.00 \%$ & 36 \\
\hline & & & Through & $10.00 \%$ & 30 \\
\hline & & & Left Turn & $78.00 \%$ & 234 \\
\hline & FH Pkwy 8B EB & 152 & Right Turn & $5.92 \%$ & 9 \\
\hline & & & Through & $57.89 \%$ & 88 \\
\hline & & & Left Turn & $36.18 \%$ & 55 \\
\hline & U.S. $321 \mathrm{SB}$ & 680 & Right Turn & $11.32 \%$ & 77 \\
\hline & & & Through & $80.15 \%$ & 545 \\
\hline & & & Left Tum & $8.53 \%$ & 58 \\
\hline & U.S. $321 \mathrm{NB}$ & 849 & Right Tum & $20.49 \%$ & 174 \\
\hline & & & Through & $79.03 \%$ & 671 \\
\hline & & & Left Turn & $0.47 \%$ & 4 \\
\hline \multirow[t]{7}{*}{ U.S. 321 Convergence w/ SR 32} & U.S. $321 \mathrm{~EB}$ & 547 & Right Turn & $12.43 \%$ & 68 \\
\hline & & & Left Tum & $87.57 \%$ & 479 \\
\hline & U.S. $321 \mathrm{SB}$ & 306 & Right Tum & $73.53 \%$ & 225 \\
\hline & & & Through & $26.47 \%$ & 81 \\
\hline & SR 32 NB & 90 & Through & $68.89 \%$ & 62 \\
\hline & & & Left Turn & $31.11 \%$ & 28 \\
\hline & CD & & & & \\
\hline \multirow[t]{6}{*}{ U.S. 321 Intersection w/ SR 416} & SR 416 SB & 176 & Right Tum & $81.25 \%$ & 143 \\
\hline & & & Left Turn & $18.75 \%$ & 33 \\
\hline & U.S. $321 \mathrm{WB}$ & 976 & Right Tum & $1.84 \%$ & 18 \\
\hline & & & Through & $98.16 \%$ & 958 \\
\hline & U.S. $321 \mathrm{~EB}$ & 470 & Through & $90.64 \%$ & 426 \\
\hline & & & Left Turn & $9.36 \%$ & 44 \\
\hline \multirow[t]{6}{*}{ SR 416 Intersection w/ Webb Creek Rd } & Webb CrRd WB & 82 & Right Tum & $3.66 \%$ & 3 \\
\hline & & & Left Turn & $96.34 \%$ & 79 \\
\hline & SR 416 SB & 65 & Through & $96.92 \%$ & 63 \\
\hline & & & Left Tum & $3.08 \%$ & 2 \\
\hline & SR 416 NB & 113 & Right Turn & $47.79 \%$ & 54 \\
\hline & & & Through & $52.21 \%$ & 59 \\
\hline \multirow[t]{12}{*}{ SR 416 Intersection w/ Foothills Pkwy 8B/8C } & FH Pkwy 8B WB & 107 & Right Turn & $0.00 \%$ & 0 \\
\hline & & & Through & $100.00 \%$ & 107 \\
\hline & & & Left Turn & $0.00 \%$ & $\mathbf{0}$ \\
\hline & FH Pkwy 8C EB & 151 & Right Turn & $0.00 \%$ & 0 \\
\hline & & & Through & $100.00 \%$ & 151 \\
\hline & & & Left Turn & $0.00 \%$ & 0 \\
\hline & SR 416 SB & 142 & Right Tum & $0.00 \%$ & 0 \\
\hline & & & Through & $100.00 \%$ & 142 \\
\hline & & & Left Turn & $0.00 \%$ & 0 \\
\hline & SR $416 \mathrm{NB}$ & 115 & Right Turn & $\quad 0.00 \%$ & 0 \\
\hline & & & Through & $100.00 \%$ & 115 \\
\hline & & & Left Turn & $0.00 \%$ & 0 \\
\hline
\end{tabular}


Table K.6. Weekday P.M. peak-hour intersection volume projections for $2026-$ construct $8 B$ with no interchanges option

\begin{tabular}{|c|c|c|c|c|c|}
\hline \multirow{2}{*}{ Intersection location } & \multirow[b]{2}{*}{ Approach } & \multirow{2}{*}{$\begin{array}{l}\text { Total } \\
\text { vehicles } \\
\text { per hour }\end{array}$} & \multicolumn{3}{|c|}{ Directional information } \\
\hline & & & $\begin{array}{c}\text { Turning } \\
\text { movement }\end{array}$ & Percent & Counts \\
\hline \multirow[t]{12}{*}{ U.S. 321 Intersection w/ Foothills Pkwy } & FH Pkwy 8A WB & 221 & Right Tum & $14.93 \%$ & 33 \\
\hline & & & Through & $9.50 \%$ & 21 \\
\hline & & & Left Turn & $75.57 \%$ & 167 \\
\hline & FH Pkwy 8B EB & 201 & Right Tum & $5.97 \%$ & 12 \\
\hline & & & Through & $57.71 \%$ & 116 \\
\hline & & & Left Turn & $36.32 \%$ & 73 \\
\hline & U.S. 321 SB & 711 & Right Tum & $12.10 \%$ & 86 \\
\hline & & & Through & $85.23 \%$ & 606 \\
\hline & & & Left Turn & $2.67 \%$ & 19 \\
\hline & U.S. $321 \mathrm{NB}$ & 867 & Right Tum & $13.49 \%$ & 117 \\
\hline & & & Through & $85.93 \%$ & 745 \\
\hline & & & Left Turn & $0.58 \%$ & 5 \\
\hline \multirow[t]{6}{*}{ U.S. 321 Convergence w/ SR 32} & U.S. $321 \mathrm{~EB}$ & 724 & Right Tum & $12.71 \%$ & 92 \\
\hline & & & Left Turn & $87.29 \%$ & 632 \\
\hline & U.S. $321 \mathrm{SB}$ & 306 & Right Tum & $74.18 \%$ & 227 \\
\hline & & & Through & $25.82 \%$ & 79 \\
\hline & SR $32 \mathrm{NB}$ & 63 & Through & $66.67 \%$ & 42 \\
\hline & & & Left Turn & $33.33 \%$ & 21 \\
\hline \multirow[t]{6}{*}{ U.S. 321 Intersection w/ SR 416} & SR $416 \mathrm{SB}$ & 115 & Right Tum & $84.35 \%$ & 97 \\
\hline & & & Left Turn & $15.65 \%$ & 18 \\
\hline & U.S. 321 WB & 944 & Right Turn & $4.13 \%$ & 39 \\
\hline & & & Through & $95.87 \%$ & 905 \\
\hline & U.S. 321 EB & 393 & Through & $93.89 \%$ & 369 \\
\hline & & & Left Turn & $6.11 \%$ & 24 \\
\hline \multirow[t]{6}{*}{ SR 416 Intersection w/ Webb Creek Rd } & Webb Cr Rd WB & 89 & Right Tum & $6.74 \%$ & 6 \\
\hline & & & Left Turn & $93.26 \%$ & 83 \\
\hline & SR $416 \mathrm{SB}$ & 80 & Through & $98.75 \%$ & 79 \\
\hline & & & Left Turn & $1.25 \%$ & 1 \\
\hline & SR $416 \mathrm{NB}$ & 146 & Right Tum & $43.84 \%$ & 64 \\
\hline & & & Through & $56.16 \%$ & 82 \\
\hline \multirow[t]{12}{*}{ SR 416 Intersection w/ Foothills Pkwy $8 \mathrm{~B} / 8 \mathrm{C}$} & FH Pkwy 8B WB & 108 & Right Tum & $0.00 \%$ & 0 \\
\hline & & & Through & $100.00 \%$ & 108 \\
\hline & & & Left Turn & $0.00 \%$ & 0 \\
\hline & FH Pkwy 8C EB & 200 & Right Turn & $0.00 \%$ & 0 \\
\hline & & & Through & $100.00 \%$ & 200 \\
\hline & & - & Left Turn & $0.00 \%$ & 0 \\
\hline & SR $416 \mathrm{SB}$ & 163 & Right Turn & $0.00 \%$ & 0 \\
\hline & & & Through & $100.00 \%$ & 163 \\
\hline & & & Left Turn & $0.00 \%$ & 0 \\
\hline & SR 416 NB & 149 & Right Turn & $0.00 \%$ & 0 \\
\hline & & & Through & $100.00 \%$ & 149 \\
\hline & & & Left Tum & $0.00 \%$ & 0 \\
\hline
\end{tabular}


Table K.7. Weekend A.M. peak-hour intersection volume projections for 2026-construct 8B with no interchanges option

\begin{tabular}{|c|c|c|c|c|c|}
\hline \multirow[b]{2}{*}{ Intersection location } & \multirow[b]{2}{*}{ Approach } & \multirow{2}{*}{$\begin{array}{c}\text { Total } \\
\text { vehicles } \\
\text { per hour }\end{array}$} & \multicolumn{3}{|c|}{ Directional information } \\
\hline & & & $\begin{array}{l}\text { Turning } \\
\text { movement }\end{array}$ & Percent & Counts \\
\hline \multirow[t]{4}{*}{ U.S. 321 Intersection w/ Foothills Pkwy } & FH Pkwy 8A WB & 507 & $\begin{array}{l}\text { Right Tum } \\
\text { Through } \\
\text { Left Tum }\end{array}$ & $\begin{array}{r}12.03 \% \\
9.86 \% \\
78.11 \%\end{array}$ & $\begin{array}{r}61 \\
50 \\
396\end{array}$ \\
\hline & FH Pkwy 8B EB & 208 & $\begin{array}{l}\text { Right Turn } \\
\text { Through } \\
\text { Left Turn }\end{array}$ & $\begin{array}{r}5.77 \% \\
57.69 \% \\
36.54 \%\end{array}$ & $\begin{array}{r}12 \\
120 \\
76\end{array}$ \\
\hline & U.S. $321 \mathrm{SB}$ & 749 & $\begin{array}{l}\text { Right Tum } \\
\text { Through } \\
\text { Left Turn }\end{array}$ & $\begin{array}{r}11.35 \% \\
79.97 \% \\
8.68 \%\end{array}$ & $\begin{array}{r}85 \\
599 \\
65\end{array}$ \\
\hline & U.S. $321 \mathrm{NB}$ & 1046 & $\begin{array}{l}\text { Right Tum } \\
\text { Through } \\
\text { Left Tum }\end{array}$ & $\begin{array}{r}20.65 \% \\
78.87 \% \\
0.48 \%\end{array}$ & $\begin{array}{r}216 \\
825 \\
5\end{array}$ \\
\hline \multirow[t]{3}{*}{ U.S. 321 Convergence w/ SR 32} & U.S. $321 \mathrm{~EB}$ & 749 & $\begin{array}{l}\text { Right Turn } \\
\text { Left Turn }\end{array}$ & $\begin{array}{l}12.42 \% \\
87.58 \%\end{array}$ & $\begin{array}{r}93 \\
656\end{array}$ \\
\hline & U.S. $321 \mathrm{SB}$ & 696 & $\begin{array}{l}\text { Right Turn } \\
\text { Through }\end{array}$ & $\begin{array}{l}73.56 \% \\
26.44 \%\end{array}$ & $\begin{array}{l}512 \\
184\end{array}$ \\
\hline & SR 32 NB & 123 & $\begin{array}{l}\text { Through } \\
\text { Left Turn }\end{array}$ & $\begin{array}{l}69.92 \% \\
30.08 \%\end{array}$ & $\begin{array}{l}86 \\
37\end{array}$ \\
\hline \multirow[t]{3}{*}{ U.S. 321 Intersection w/ SR 416} & SR 416 SB & 191 & $\begin{array}{l}\text { Right Tum } \\
\text { Left Tum }\end{array}$ & $\begin{array}{l}81.68 \% \\
18.32 \%\end{array}$ & $\begin{array}{r}156 \\
35\end{array}$ \\
\hline & U.S. 321 WB & 1185 & $\begin{array}{l}\text { Right Tum } \\
\text { Through }\end{array}$ & $\begin{array}{r}1.94 \% \\
98.06 \%\end{array}$ & $\begin{array}{r}23 \\
1162\end{array}$ \\
\hline & U.S. $321 \mathrm{~EB}$ & 598 & $\begin{array}{l}\text { Through } \\
\text { Left Turn }\end{array}$ & $\begin{array}{r}90.47 \% \\
9.53 \%\end{array}$ & $\begin{array}{r}541 \\
57\end{array}$ \\
\hline \multirow[t]{3}{*}{ SR 416 Intersection w/ Webb Creek Rd } & Webb Cr Rd WB & 52 & $\begin{array}{l}\text { Right Turn } \\
\text { Left Turn }\end{array}$ & $\begin{array}{r}3.85 \% \\
96.15 \%\end{array}$ & $\begin{array}{r}2 \\
50\end{array}$ \\
\hline & SR $416 \mathrm{SB}$ & 48 & $\begin{array}{l}\text { Through } \\
\text { Left Turn }\end{array}$ & $\begin{array}{r}95.83 \% \\
4.17 \%\end{array}$ & $\begin{array}{r}46 \\
2\end{array}$ \\
\hline & SR $416 \mathrm{NB}$ & 94 & $\begin{array}{l}\text { Right Turn } \\
\text { Through }\end{array}$ & $\begin{array}{l}46.81 \% \\
53.19 \%\end{array}$ & $\begin{array}{l}44 \\
50\end{array}$ \\
\hline \multirow[t]{4}{*}{ SR 416 Intersection w/ Foothills Pkwy $8 \mathrm{~B} / 8 \mathrm{C}$} & FH Pkwy 8B WB & 139 & $\begin{array}{l}\text { Right Tum } \\
\text { Through } \\
\text { Left Turn }\end{array}$ & $\begin{array}{r}0.00 \% \\
100.00 \% \\
0.00 \%\end{array}$ & 139 \\
\hline & FH Pkwy 8C EB & 207 & $\begin{array}{l}\text { Right Turn } \\
\text { Through } \\
\text { Left Turn }\end{array}$ & $\begin{array}{r}0.00 \% \\
100.00 \% \\
0.00 \%\end{array}$ & 207 \\
\hline & SR $416 \mathrm{SB}$ & 96 & $\begin{array}{l}\text { Right Turn } \\
\text { Through } \\
\text { Left Tum }\end{array}$ & $\begin{array}{r}0.00 \% \\
100.00 \% \\
0.00 \%\end{array}$ & 96 \\
\hline & SR $416 \mathrm{NB}$ & 96 & $\begin{array}{l}\text { Right Turn } \\
\text { Through } \\
\text { Left Tum }\end{array}$ & $\begin{array}{r}0.00 \% \\
100.00 \% \\
0.00 \%\end{array}$ & 96 \\
\hline
\end{tabular}


Table K.8. Weekend P.M. peak-hour intersection volume projections for 2026 -construct 8B with no interchanges option

\begin{tabular}{|c|c|c|c|c|c|}
\hline & & & Dir & al informat & \\
\hline Intersection location & Approach & $\begin{array}{l}\text { vehicles } \\
\text { per hour }\end{array}$ & $\begin{array}{c}\text { Turning } \\
\text { movement }\end{array}$ & Percent & Counts \\
\hline U.S. 321 Intersection w/ Foothills Pkwy & FH Pkwy 8A WB & 308 & Right Turn & $15.26 \%$ & 47 \\
\hline & & & Through & $9.42 \%$ & 29 \\
\hline & & & Left Turn & $75.32 \%$ & 232 \\
\hline & FH Pkwy 8B EB & 241 & Right Turn & $5.81 \%$ & 14 \\
\hline & & & Through & $57.68 \%$ & 139 \\
\hline & & & Left Turn & $36.51 \%$ & 88 \\
\hline & U.S. $321 \mathrm{SB}$ & 833 & Right Turn & $12.12 \%$ & 101 \\
\hline & & & Through & $85.23 \%$ & 710 \\
\hline & & & Left Tum & $2.64 \%$ & 22 \\
\hline & U.S. $321 \mathrm{NB}$ & 991 & Right Tum & $13.42 \%$ & 133 \\
\hline & & & Through & $86.07 \%$ & 853 \\
\hline & & & Left Turn & $0.50 \%$ & 5 \\
\hline U.S. 321 Convergence w/ SR 32 & U.S. $321 \mathrm{~EB}$ & 871 & Right Turn & $12.74 \%$ & 111 \\
\hline & & & Left Turn & $87.26 \%$ & 760 \\
\hline & U.S. $321 \mathrm{SB}$ & 580 & Right Tum & $73.97 \%$ & 429 \\
\hline & & & Through & $26.03 \%$ & 151 \\
\hline & SR 32 NB & 82 & Through & $65.85 \%$ & 54 \\
\hline & & & Left Turn & $34.15 \%$ & 28 \\
\hline U.S. 321 Intersection w/ SR 416 & SR $416 \mathrm{SB}$ & 224 & Right Tum & $83.48 \%$ & 187 \\
\hline & & & Left Turn & $16.52 \%$ & 37 \\
\hline . & U.S. 321 WB & 1263 & Right Turn & $4.20 \%$ & 53 \\
\hline & & & Through & $95.80 \%$ & 1210 \\
\hline & U.S. $321 \mathrm{~EB}$ & 560 & Through & $93.93 \%$ & 526 \\
\hline & & & Left Turn & $6.07 \%$ & 34 \\
\hline SR 416 Intersection w/ Webb Creek Rd & Webb Cr Rd WB & 68 & Right Turn & $7.35 \%$ & 5 \\
\hline & & & Left Turn & $92.65 \%$ & 63 \\
\hline & SR $416 \mathrm{SB}$ & 110 & Through & $99.09 \%$ & 109 \\
\hline & & & Left Turn & $0.91 \%$ & 1 \\
\hline & SR $416 \mathrm{NB}$ & 146 & Right Turn & $43.84 \%$ & 64 \\
\hline & & & Through & $56.16 \%$ & 82 \\
\hline SR 416 Intersection w/ Foothills Pkwy 8B/8C & FH Pkwy 8B WB & 130 & Right Turn & $0.00 \%$ & 0 \\
\hline & & & Through & $100.00 \%$ & 130 \\
\hline & & & Left Turn & $0.00 \%$ & 0 \\
\hline & FH Pkwy 8C EB & 240 & Right Turn & $0.00 \%$ & \\
\hline & & & Through & $100.00 \%$ & 240 \\
\hline & & & Left Turn & $0.00 \%$ & 0 \\
\hline & SR 416 SB & 171 & Right Turn & $0.00 \%$ & 0 \\
\hline & & & Through & $100.00 \%$ & 171 \\
\hline & & & Left Turn & $0.00 \%$ & 0 \\
\hline . & SR 416 NB & 149 & Right Turn & $0.00 \%$ & 0 \\
\hline & & & Through & $100.00 \%$ & 149 \\
\hline & & & Left Tum & $0.00 \%$ & 0 \\
\hline
\end{tabular}


Table K.9. Weekday A.M. peak-hour intersection volume projections for 2006-interchange at Highway 416 (north ramp option)-8B not opened until $8 \mathrm{C}$ completed

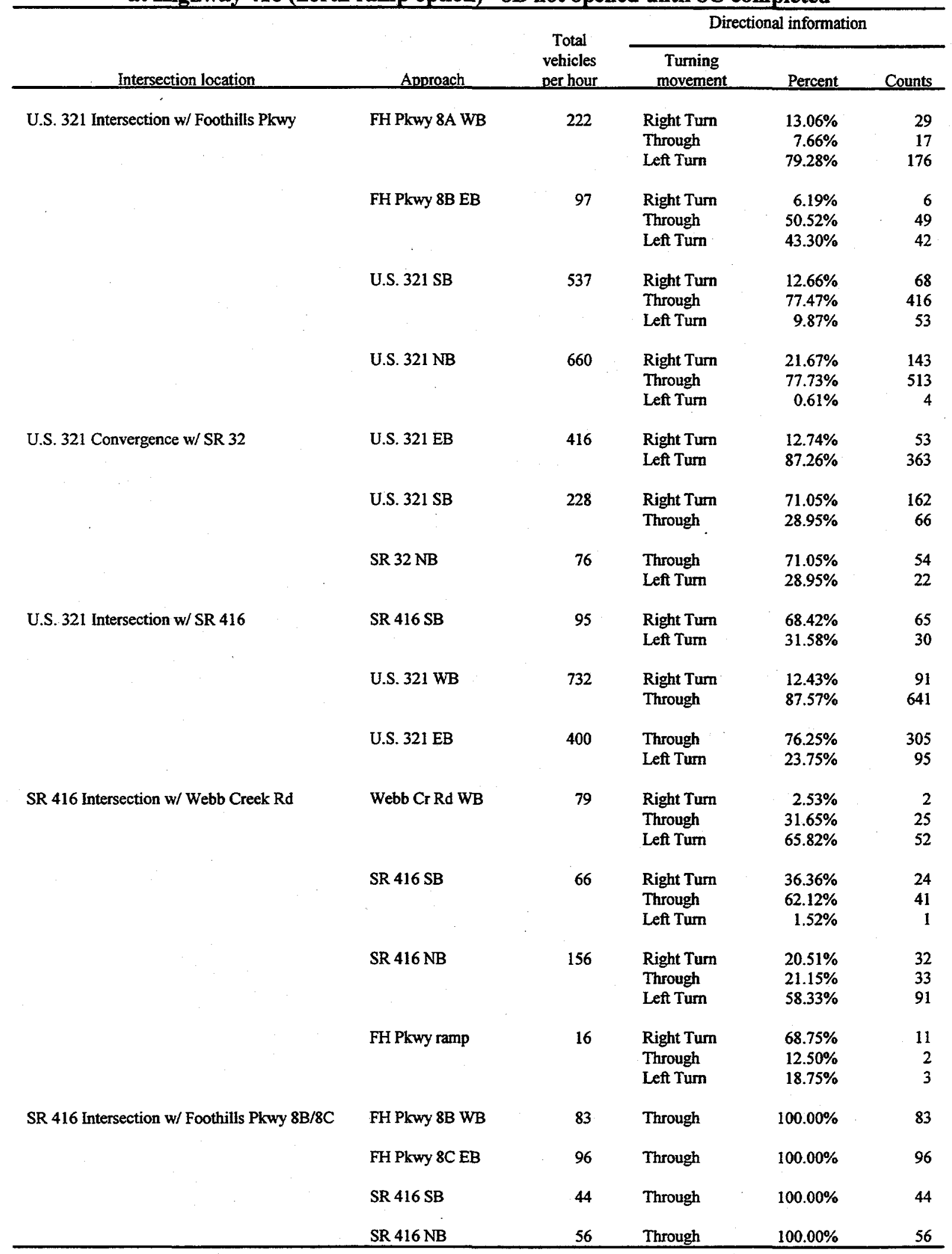


Table K.10. Weekday P.M. peak-hour intersection volume projections for 2006 -interchange at Highway 416 (north ramp option) $-8 B$ not opened until $8 \mathrm{C}$ completed

\begin{tabular}{|c|c|c|c|c|c|}
\hline \multirow{2}{*}{ Intersection location } & \multirow[b]{2}{*}{ Approach } & \multirow{2}{*}{$\begin{array}{l}\text { Total } \\
\text { vehicles } \\
\text { per hour }\end{array}$} & \multicolumn{3}{|c|}{ Directional information } \\
\hline & & & $\begin{array}{c}\text { Turning } \\
\text { movement }\end{array}$ & Percent & Counts \\
\hline \multirow[t]{12}{*}{ U.S. 321 Intersection w/ Foothills Pkwy } & FH Pkwy 8A wB & 163 & Right Tum & $15.95 \%$ & 26 \\
\hline & & & Through & $7.36 \%$ & 12 \\
\hline & & & Left Tum & $76.69 \%$ & 125 \\
\hline & FH Pkwy 8B EB & 128 & Right Turn & $6.25 \%$ & 8 \\
\hline & & & Through & $50.78 \%$ & 65 \\
\hline & & & Left Turn & $42.97 \%$ & 55 \\
\hline & U.S. 321 SB & 555 & Right Turn & $13.69 \%$ & 76 \\
\hline & & & Through & $83.24 \%$ & 462 \\
\hline & & & Left Turn & $3.06 \%$ & 17 \\
\hline & U.S. $321 \mathrm{NB}$ & 671 & Right Tum & $14.31 \%$ & 96 \\
\hline & & & Through & $84.95 \%$ & 570 \\
\hline & & & Left Turn & $0.75 \%$ & 5 \\
\hline \multirow[t]{6}{*}{ U.S. 321 Convergence w/ SR 32} & U.S. $321 \mathrm{~EB}$ & 551 & Right Tum & $13.07 \%$ & 72 \\
\hline & & & Left Turn & $86.93 \%$ & 479 \\
\hline & U.S. $321 \mathrm{SB}$ & 228 & Right Tum & $71.49 \%$ & 163 \\
\hline & & & Through & $28.51 \%$ & 65 \\
\hline & SR 32 NB & 54 & Through & $68.52 \%$ & 37 \\
\hline & & & Left Turn & $31.48 \%$ & 17 \\
\hline \multirow[t]{6}{*}{ U.S. 321 Intersection w/ SR 416} & SR $416 \mathrm{SB}$ & 61 & Right Turn & $72.13 \%$ & 44 \\
\hline & & & Left Turn & $27.87 \%$ & 17 \\
\hline & U.S. 321 WB & 804 & Right Turn & $24.75 \%$ & 199 \\
\hline & & & Through & $75.25 \%$ & 605 \\
\hline & U.S. $321 \mathrm{~EB}$ & 316 & Through & $83.54 \%$ & 264 \\
\hline & & & Left Turn & $16.46 \%$ & 52 \\
\hline \multirow[t]{12}{*}{ SR 416 Intersection w/ Webb Creek Rd } & Webb Cr Rd WB & 86 & Right Tum & $3.49 \%$ & 3 \\
\hline & & & Through & $32.56 \%$ & 28 \\
\hline & & & Left Turn & $63.95 \%$ & 55 \\
\hline & SR $416 \mathrm{SB}$ & 81 & Right Turn & $34.57 \%$ & 28 \\
\hline & & & Through & $64.20 \%$ & 52 \\
\hline & & & Left Turn & $1.23 \%$ & 1 \\
\hline & SR 416 NB & 202 & Right Tum & $18.81 \%$ & 38 \\
\hline & & & Through & $22.77 \%$ & 46 \\
\hline & & & Left Turn & $58.42 \%$ & 118 \\
\hline & FH Pkwy ramp & 21 & Right Tum & $66.67 \%$ & 14 \\
\hline & & & Through & $14.29 \%$ & 3 \\
\hline & & & Left Turn & $19.05 \%$ & 4 \\
\hline \multirow[t]{4}{*}{ SR 416 Intersection w/ Foothills Pkwy 8B/8C } & FH Pkwy 8B WB & 84 & Through & $100.00 \%$ & 84 \\
\hline & FH Pkwy 8C EB & 126 & Through & $100.00 \%$ & 126 \\
\hline & SR 416 SB & 51 & Through & $100.00 \%$ & 51 \\
\hline & SR $416 \mathrm{NB}$ & 73 & Through & $100.00 \%$ & 73 \\
\hline
\end{tabular}


Table K.11. Weekend A.M. peak-hour intersection volume projections for 2006-interchange at Highway 416 (north ramp option)-8B not opened until 8C completed

\begin{tabular}{|c|c|c|c|c|c|}
\hline \multirow[b]{2}{*}{ Intersection location } & \multirow[b]{2}{*}{ Approach } & \multirow{2}{*}{$\begin{array}{c}\text { Total } \\
\text { vehicles } \\
\text { per hour }\end{array}$} & \multicolumn{3}{|c|}{ Directional information } \\
\hline & & & $\begin{array}{c}\text { Tuming } \\
\text { movement }\end{array}$ & Percent & Counts \\
\hline \multirow[t]{12}{*}{ U.S. 321 Intersection w/ Foothills Pkwy } & FH Pkwy 8A WB & 375 & Right Tum & $13.07 \%$ & 49 \\
\hline & & & Through & $7.73 \%$ & 29 \\
\hline & & & Left Turn & $79.20 \%$ & 297 \\
\hline & FH Pkwy 8B EB & 132 & Right Tum & $6.06 \%$ & 8 \\
\hline & & & Through & $50.76 \%$ & 67 \\
\hline & & & Left Turn & $43.18 \%$ & 57 \\
\hline & U.S. $321 \mathrm{SB}$ & 591 & Right Turn & $12.69 \%$ & 75 \\
\hline & & & Through & $77.33 \%$ & 457 \\
\hline & & & Left Tum & $9.98 \%$ & 59 \\
\hline & U.S. $321 \mathrm{NB}$ & 813 & Right Turn & $21.77 \%$ & 177 \\
\hline & & & Through & $77.61 \%$ & 631 \\
\hline & & & Left Turn & $0.62 \%$ & 5 \\
\hline \multirow[t]{6}{*}{ U.S. 321 Convergence $w / S R 32$} & U.S. $321 \mathrm{~EB}$ & 570 & Right Tum & $12.81 \%$ & 73 \\
\hline & & & Left Turn & $87.19 \%$ & 497 \\
\hline & U.S. $321 \mathrm{SB}$ & 519 & Right Tum & $70.91 \%$ & 368 \\
\hline & & & Through & $29.09 \%$ & 151 \\
\hline & SR.32 NB & 104 & Through & $72.12 \%$ & 75 \\
\hline & & & Left Turn & $27.88 \%$ & 29 \\
\hline \multirow[t]{6}{*}{ U.S. 321 Intersection w/SR 416} & SR 416 SB & 103 & Right Turn & $68.93 \%$ & 71 \\
\hline & & & Left Tum & $31.07 \%$ & 32 \\
\hline & U.S. 321 WB & 893 & Right Tum & $12.99 \%$ & 116 \\
\hline & & & Through & $87.01 \%$ & 777 . \\
\hline & U.S. $321 \mathrm{~EB}$ & 509 & Through & $76.03 \%$ & 387 \\
\hline & & & Left Tum & $23.97 \%$ & 122 \\
\hline \multirow[t]{12}{*}{ SR 416 Intersection w/ Webb Creek Rd } & Webb Cr Rd WB & 52 & Right Turn & $1.92 \%$ & 1 \\
\hline & & & Through & $34.62 \%$ & 18 \\
\hline & & & Left Tum & $63.46 \%$ & 33 \\
\hline & SR 416 SB & 46 & Right Turn & $32.61 \%$ & 15 \\
\hline & & & Through & $65.22 \%$ & 30 \\
\hline & & & Left Turn & $2.17 \%$ & 1 \\
\hline & SR 416 NB & 130 & Right Turn & $20.00 \%$ & 26 \\
\hline & & & Through & $21.54 \%$ & 28 \\
\hline & & & Left Turn & $58.46 \%$ & 76 \\
\hline & FH Pkwy ramp & 22 & Right Turn & $68.18 \%$ & 15 \\
\hline & & & Through & $13.64 \%$ & 3 \\
\hline & & & Left Tum & $18.18 \%$ & 4 \\
\hline \multirow[t]{4}{*}{ SR 416 Intersection w/ Foothills Pkwy 8B/8C } & FH Pkwy 8B wB & 108 & Through & $100.00 \%$ & 108 \\
\hline & FH Pkwy 8C EB & 131 & Through & $100.00 \%$ & 131 \\
\hline & SR 416 SB & 30 & Through & $100.00 \%$ & 30 \\
\hline & SR 416 NB & 47 & Through & $100.00 \%$ & 47 \\
\hline
\end{tabular}


Table K.12. Weekend P.M. peak-hour intersection volume projections for 2006-interchange at Highway 416 (north ramp option)-8B not opened until 8C completed

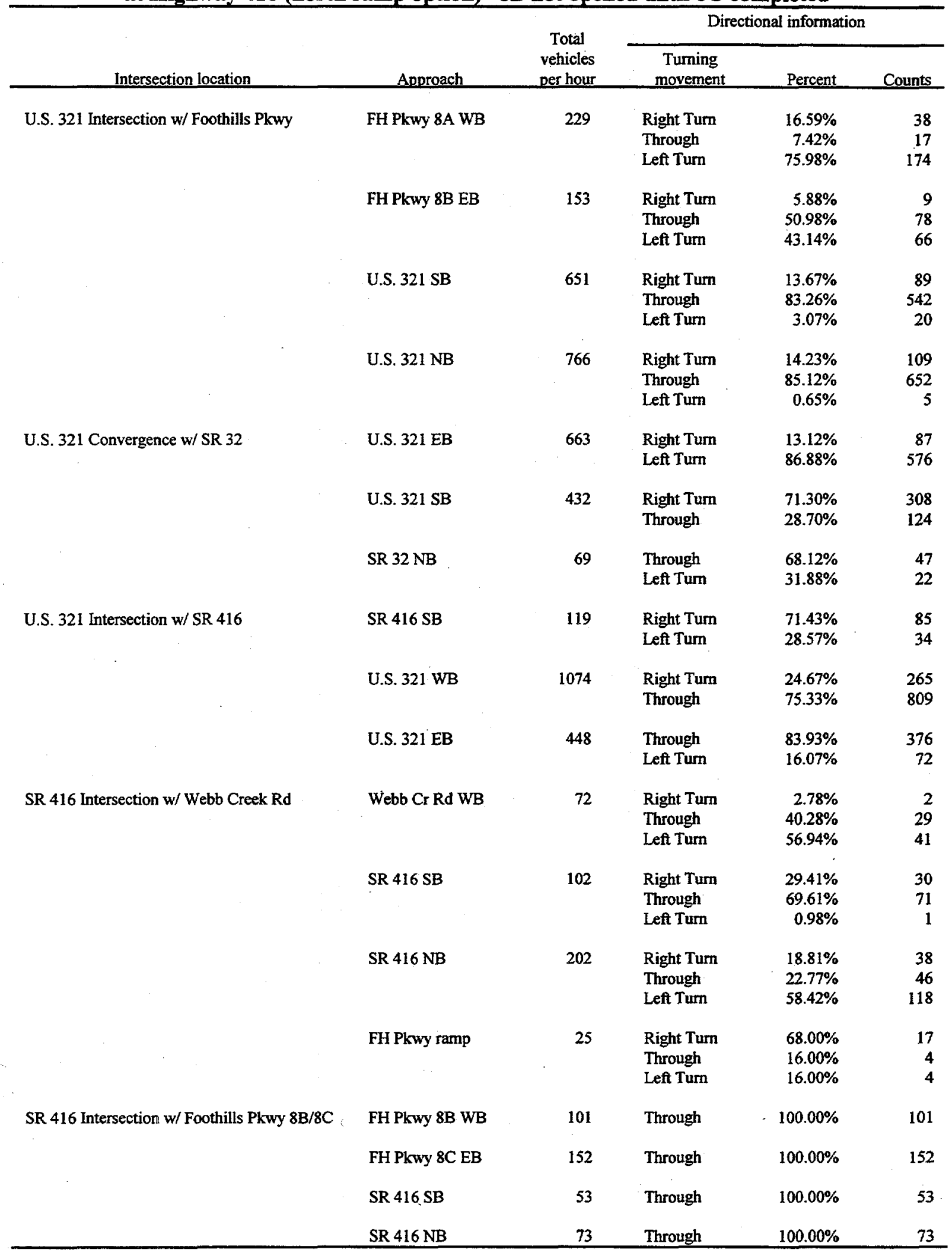


Table K.13. Weekday A.M. peak-hour intersection volume projections for 2026 -interchange at Highway 416 (north ramp option) $-8 \mathrm{~B}$ not opened until $8 \mathrm{C}$ completed

\begin{tabular}{|c|c|c|c|c|c|}
\hline \multirow[b]{2}{*}{ Intersection location } & \multirow[b]{2}{*}{ Approach } & \multirow{2}{*}{$\begin{array}{c}\text { Total } \\
\text { vehicles } \\
\text { per hour }\end{array}$} & \multicolumn{3}{|c|}{ Directional information } \\
\hline & & & $\begin{array}{c}\text { Tuming } \\
\text { movement }\end{array}$ & Percent & Counts \\
\hline \multirow[t]{12}{*}{ U.S. 321 Intersection w/ Foothills Pkwy } & FH Pkwy 8A WB & 300 & Right Turn & $12.00 \%$ & 36 \\
\hline & & & Through & $10.00 \%$ & 30 \\
\hline & & & Left Tum & $78.00 \%$ & 234 \\
\hline & FH Pkwy 8B EB & 152 & Right Turn & $5.92 \%$ & 9 \\
\hline & & & Through & $57.89 \%$ & 88 \\
\hline & & & Left Tum & $36.18 \%$ & 55 \\
\hline & U.S. 321 SB & 680 & Right Turn & $11.32 \%$ & 77 \\
\hline & & & Through & $80.15 \%$ & 545 \\
\hline & & & Left Tum & $8.53 \%$ & 58 \\
\hline & U.S. $321 \mathrm{NB}$ & 849 & Right Turn & $20.49 \%$ & 174 \\
\hline & & & Through & $79.03 \%$ & 671 \\
\hline & & & Left Tum & $0.47 \%$ & 4 \\
\hline \multirow[t]{6}{*}{ U.S. 321 Convergence w/ SR 32} & U.S. $321 \mathrm{~EB}$ & 547 & Right Turn & $12.43 \%$ & 68 \\
\hline & & . & Left Turn & $87.57 \%$ & 479 \\
\hline & U.S. $321 \mathrm{SB}$ & 306 & Right Tum & $73.53 \%$ & 225 \\
\hline & & & Through & $26.47 \%$ & 81 \\
\hline & SR 32 NB & 90 & Through & $68.89 \%$ & 62 \\
\hline & & & Left Tum & $31.11 \%$ & 28 \\
\hline \multirow[t]{6}{*}{ U.S. 321 Intersection w/ SR 416} & SR 416 SB & 121 & Right Turn & $72.73 \%$ & 88 \\
\hline & & & Left Tum & $27.27 \%$ & 33 \\
\hline & U.S. $321 \mathrm{WB}$ & 972 & Right Turn & $8.44 \%$ & 82 \\
\hline & & & Through & $91.56 \%$ & 890 \\
\hline & U.S. $321 \mathrm{~EB}$ & 455 & Through & $93.63 \%$ & 426 \\
\hline & & & Left Turn & $6.37 \%$ & 29 \\
\hline \multirow[t]{12}{*}{ SR 416 Intersection $w /$ Webb Creek Rd } & Webb Cr Rd WB & 127 & Right Turn & $2.36 \%$ & 3 \\
\hline & & & Through & $35.43 \%$ & 45 \\
\hline & & & Left Tum & $62.20 \%$ & 79 \\
\hline & SR $416 \mathrm{SB}$ & 109 & Right Turn & $40.37 \%$ & 44 \\
\hline & & & Through & $57.80 \%$ & 63 \\
\hline & & & Left Turn & $1.83 \%$ & 2 \\
\hline & SR $416 \mathrm{NB}$ & 159 & Right Turn . & $0.63 \%$ & 1 \\
\hline & & & Through & $37.11 \%$ & 59 \\
\hline & & & Left Tum & $62.26 \%$ & 99 \\
\hline & FH Pkwy ramp & 15 & Right Turn & $6.67 \%$ & 1 \\
\hline & & & Through & $46.67 \%$ & 7 \\
\hline & & & Left Turn & $46.67 \%$ & 7 \\
\hline \multirow[t]{4}{*}{ SR 416 Intersection w/ Foothills Pkwy 8B/8C } & FH Pkwy 8B WB & 107 & Through & $100.00 \%$ & 107 \\
\hline & FH Pkwy 8C EB & 151 & Through & $100.00 \%$ & 151 \\
\hline & SR 416 SB & 53 & Through & $100.00 \%$ & 53 \\
\hline & SR $416 \mathrm{NB}$ & 90 & Through & $100.00 \%$ & 90 \\
\hline
\end{tabular}


Table K.14. Weekday P.M. peak-hour intersection volume projections for 2026-interchange at Highway 416 (north ramp option)-8B not opened until 8C completed

\begin{tabular}{|c|c|c|c|c|c|}
\hline \multirow[b]{2}{*}{ Intersection location } & \multirow[b]{2}{*}{ Approach } & \multirow{2}{*}{$\begin{array}{c}\text { Total } \\
\text { vehicles } \\
\text { per hour }\end{array}$} & \multicolumn{3}{|c|}{ Directional information } \\
\hline & & & $\begin{array}{c}\text { Turning } \\
\text { movement }\end{array}$ & Percent & Counts \\
\hline \multirow[t]{4}{*}{ U.S. 321 Intersection w/ Foothills Pkwy } & FH Pkwy 8A WB & 221 & $\begin{array}{l}\text { Right Turn } \\
\text { Through } \\
\text { Left Tum }\end{array}$ & $\begin{array}{r}14.93 \% \\
9.50 \% \\
75.57 \%\end{array}$ & $\begin{array}{r}33 \\
21 \\
167\end{array}$ \\
\hline & FH Pkwy 8B EB & 201 & $\begin{array}{l}\text { Right Tum } \\
\text { Through } \\
\text { Left Turn }\end{array}$ & $\begin{array}{r}5.97 \% \\
57.71 \% \\
36.32 \%\end{array}$ & $\begin{array}{r}12 \\
116 \\
73\end{array}$ \\
\hline & U.S. 321 SB & 711 & $\begin{array}{l}\text { Right Turn } \\
\text { Through } \\
\text { Left Turn }\end{array}$ & $\begin{array}{r}12.10 \% \\
85.23 \% \\
2.67 \%\end{array}$ & $\begin{array}{r}86 \\
606 \\
19\end{array}$ \\
\hline & U.S. $321 \mathrm{NB}$ & 867 & $\begin{array}{l}\text { Right Turn } \\
\text { Through } \\
\text { Left Tum }\end{array}$ & $\begin{array}{r}13.49 \% \\
85.93 \% \\
0.58 \%\end{array}$ & $\begin{array}{r}117 \\
745 \\
5\end{array}$ \\
\hline \multirow[t]{3}{*}{ U.S. 321 Convergence w/ SR 32} & U.S. $321 \mathrm{~EB}$ & 724 & $\begin{array}{l}\text { Right Tum } \\
\text { Left Turn }\end{array}$ & $\begin{array}{l}12.71 \% \\
87.29 \%\end{array}$ & $\begin{array}{r}92 \\
632\end{array}$ \\
\hline & U.S. $321 \mathrm{SB}$ & 306 & $\begin{array}{l}\text { Right Turn } \\
\text { Through }\end{array}$ & $\begin{array}{l}74.18 \% \\
25.82 \%\end{array}$ & $\begin{array}{r}227 \\
79\end{array}$ \\
\hline & SR $32 \mathrm{NB}$ & 63 & $\begin{array}{l}\text { Through } \\
\text { Left Tum }\end{array}$ & $\begin{array}{l}66.67 \% \\
33.33 \%\end{array}$ & $\begin{array}{l}42 \\
21\end{array}$ \\
\hline \multirow[t]{3}{*}{ U.S. 321 Intersection w/ SR 416} & SR $416 \mathrm{SB}$ & 78 & $\begin{array}{l}\text { Right Turn } \\
\text { Left Turn }\end{array}$ & $\begin{array}{l}76.92 \% \\
23.08 \%\end{array}$ & $\begin{array}{l}60 \\
18\end{array}$ \\
\hline & U.S. $321 \mathrm{WB}$ & 1019 & $\begin{array}{l}\text { Right Turn } \\
\text { Through }\end{array}$ & $\begin{array}{l}17.47 \% \\
82.53 \%\end{array}$ & $\begin{array}{l}178 \\
841\end{array}$ \\
\hline & U.S. $321 \mathrm{~EB}$ & 385 & $\begin{array}{l}\text { Through } \\
\text { Left Tum }\end{array}$ & $\begin{array}{r}95.84 \% \\
4.16 \%\end{array}$ & $\begin{array}{r}369 \\
16\end{array}$ \\
\hline \multirow[t]{4}{*}{ SR 416 Intersection w/Webb Creek Rd } & Webb Cr Rd WB & 140 & $\begin{array}{l}\text { Right Turn } \\
\text { Through } \\
\text { Left Turn }\end{array}$ & $\begin{array}{r}4.29 \% \\
36.43 \% \\
59.29 \%\end{array}$ & $\begin{array}{r}6 \\
51 \\
83\end{array}$ \\
\hline & SR $416 \mathrm{SB}$ & 131 & $\begin{array}{l}\text { Right Turn } \\
\text { Through } \\
\text { Left Turn }\end{array}$ & $\begin{array}{r}38.93 \% \\
60.31 \% \\
0.76 \%\end{array}$ & $\begin{array}{r}51 \\
79 \\
1\end{array}$ \\
\hline & SR $416 \mathrm{NB}$ & 275 & $\begin{array}{l}\text { Right Turn } \\
\text { Through } \\
\text { Left Tum }\end{array}$ & $\begin{array}{l}23.27 \% \\
29.82 \% \\
46.91 \%\end{array}$ & $\begin{array}{r}64 \\
82 \\
129\end{array}$ \\
\hline & FH Pkwy ramp & 19 & $\begin{array}{l}\text { Right Turn } \\
\text { Through } \\
\text { Left Turn }\end{array}$ & $\begin{array}{r}5.26 \% \\
47.37 \% \\
47.37 \%\end{array}$ & $\begin{array}{l}1 \\
9 \\
9\end{array}$ \\
\hline \multirow[t]{4}{*}{ SR 416 Intersection w/ Foothills Pkwy $8 \mathrm{~B} / 8 \mathrm{C}$} & FH Pkwy 8B WB & 108 & Through & $100.00 \%$ & 108 \\
\hline & FH Pkwy 8C EB & 200 & Through & $100.00 \%$ & 200 \\
\hline & SR $416 \mathrm{SB}$ & 61 & Through & $100.00 \%$ & 61 \\
\hline & SR 416 NB & 116 & Through & $100.00 \%$ & 116 \\
\hline
\end{tabular}


Table K.15. Weekend A.M. peak-hour intersection volume projections for 2026-interchange at Highway 416 (north ramp option) $-8 B$ not opened until $8 \mathrm{C}$ completed

\begin{tabular}{|c|c|c|c|c|c|}
\hline \multirow[b]{2}{*}{ Intersection location } & \multirow[b]{2}{*}{ Approach } & \multirow{2}{*}{$\begin{array}{c}\text { Total } \\
\text { vehicles } \\
\text { per hour }\end{array}$} & \multicolumn{3}{|c|}{ Directional information } \\
\hline & & & $\begin{array}{l}\text { Turning } \\
\text { movement }\end{array}$ & Percent & Counts \\
\hline \multirow[t]{12}{*}{ U.S. 321 Intersection w/ Foothills Pkwy } & FH Pkwy 8A WB & 507 & Right Turn & $12.03 \%$ & 61 \\
\hline & & & Through & $9.86 \%$ & 50 \\
\hline & & & Left Turn & $78.11 \%$ & 396 \\
\hline & FH Pkwy 8B EB & 208 & Right Turn & $5.77 \%$ & 12 \\
\hline & & & Through & $57.69 \%$ & 120 \\
\hline & & & Left Turn & $36.54 \%$ & 76 \\
\hline & U.S. $321 \mathrm{SB}$ & 749 & Right Tum & $11.35 \%$ & 85 \\
\hline & & & Through & $79.97 \%$ & 599 \\
\hline & & & Left Turn & $8.68 \%$ & 65 \\
\hline & U.S. $321 \mathrm{NB}$ & 1046 & Right Turn & $20.65 \%$ & 216 \\
\hline & & & Through & $78.87 \%$ & 825 \\
\hline & & & Left Turn & $0.48 \%$ & 5 \\
\hline \multirow[t]{6}{*}{ U.S. 321 Convergence w/ SR 32} & U.S. $321 \mathrm{~EB}$ & 749 & Right Tum & $12.42 \%$ & 93 \\
\hline & & & Left Turn & $87.58 \%$ & 656 \\
\hline & U.S. $321 \mathrm{SB}$ & 696 & Right Turn & $73.56 \%$ & 512 \\
\hline & & & Through & $26.44 \%$ & 184 \\
\hline & SR 32 NB & 123 & Through & $69.92 \%$ & 86 \\
\hline & & & Left Turn & $30.08 \%$ & 37 \\
\hline \multirow[t]{6}{*}{ U.S. 321 Intersection w/ SR 416} & SR $416 \mathrm{SB}$ & 131 & Right Tum & $73.28 \%$ & 96 \\
\hline & & & Left Turn & $26.72 \%$ & 35 \\
\hline & U.S. 321 WB & 1184 & Right Turn & $8.78 \%$ & 104 \\
\hline & & & Through & $91.22 \%$ & 1080 \\
\hline & U.S. $321 \mathrm{~EB}$ & 578 & Through & $93.60 \%$ & 541 \\
\hline & & & Left Turn & $6.40 \%$ & 37 \\
\hline \multirow[t]{12}{*}{ SR 416 Intersection w/ Webb Creek Rd } & Webb Cr Rd WB & 82 & Right Tum & $2.44 \%$ & 2 \\
\hline & & & Through & $36.59 \%$ & 30 \\
\hline & & & Left Turn & $60.98 \%$ & 50 \\
\hline & SR $416 \mathrm{SB}$ & 78 & Right Turn & $38.46 \%$ & 30 \\
\hline & & & Through & $58.97 \%$ & 46 \\
\hline & & & Left Tum & $2.56 \%$ & 2 \\
\hline & SR $416 \mathrm{NB}$ & 177 & Right Tum & $24.86 \%$ & 44 \\
\hline & & & Through & $28.25 \%$ & 50 \\
\hline & & & Left Turn & $46.89 \%$ & 83 \\
\hline & FH Pkwy ramp & 20 & Right Turn & $5.00 \%$ & 1 \\
\hline & & & Through & $45.00 \%$ & 9 \\
\hline & & & Left Turn & $50.00 \%$ & 10 \\
\hline \multirow[t]{4}{*}{ SR 416 Intersection w/ Foothills Pkwy 8B/8C } & FH Pkwy 8B WB & 139 & Through & $100.00 \%$ & 139 \\
\hline & FH Pkwy 8C EB & 207 & Through & $100.00 \%$ & 207 \\
\hline & SR 416 SB & 36 & Through & $100.00 \%$ & 36 \\
\hline & SR 416 NB & 75 & Through & $100.00 \%$ & 75 \\
\hline
\end{tabular}


Table K.16. Weekend P.M. peak-hour intersection volume projections for 2026-interchange at Highway 416 (north ramp option)-8B not opened until $8 \mathrm{C}$ completed

\begin{tabular}{|c|c|c|c|c|c|}
\hline \multirow[b]{2}{*}{ Intersection location } & \multirow[b]{2}{*}{ Approach } & \multirow{2}{*}{$\begin{array}{l}\text { Total } \\
\text { vehicles } \\
\text { per hour }\end{array}$} & \multicolumn{3}{|c|}{ Directional information } \\
\hline & & & $\begin{array}{c}\text { Turning } \\
\text { movement }\end{array}$ & Percent & Counts \\
\hline \multirow[t]{3}{*}{ U.S. 321 Intersection w/ Foothills Pkwy } & FH Pkwy 8A WB & 308 & $\begin{array}{l}\text { Right Tum } \\
\text { Through } \\
\text { Left Turn }\end{array}$ & $\begin{array}{r}15.26 \% \\
9.42 \% \\
75.32 \%\end{array}$ & $\begin{array}{r}47 \\
29 \\
232\end{array}$ \\
\hline & FH Pkwy 8B EB & 241 & $\begin{array}{l}\text { Right Turn } \\
\text { Through } \\
\text { Left Tum }\end{array}$ & $\begin{array}{r}5.81 \% \\
57.68 \% \\
36.51 \%\end{array}$ & $\begin{array}{r}14 \\
139 \\
88\end{array}$ \\
\hline & U.S. $321 \mathrm{SB}$ & 833 & $\begin{array}{l}\text { Right Tum } \\
\text { Through } \\
\text { Left Turn }\end{array}$ & $\begin{array}{r}12.12 \% \\
85.23 \% \\
2.64 \%\end{array}$ & $\begin{array}{r}101 \\
710 \\
22\end{array}$ \\
\hline & U.S. $321 \mathrm{NB}$ & 991 & $\begin{array}{l}\text { Right Tum } \\
\text { Through } \\
\text { Left Tum }\end{array}$ & $\begin{array}{r}13.42 \% \\
86.07 \% \\
0.50 \%\end{array}$ & $\begin{array}{r}133 \\
853 \\
5\end{array}$ \\
\hline \multirow[t]{3}{*}{ U.S. 321 Convergence w/SR 32} & U.S. $321 \mathrm{~EB}$ & 871 & $\begin{array}{l}\text { Right Turn } \\
\text { Left Turn }\end{array}$ & $\begin{array}{l}12.74 \% \\
87.26 \%\end{array}$ & $\begin{array}{l}111 \\
760\end{array}$ \\
\hline & U.S. $321 \mathrm{SB}$ & 580 & $\begin{array}{l}\text { Right Turn } \\
\text { Through }\end{array}$ & $\begin{array}{l}73.97 \% \\
26.03 \%\end{array}$ & $\begin{array}{l}429 \\
151\end{array}$ \\
\hline & SR $32 \mathrm{NB}$ & 82 & $\begin{array}{l}\text { Through } \\
\text { Left Turn }\end{array}$ & $\begin{array}{l}65.85 \% \\
34.15 \%\end{array}$ & $\begin{array}{l}54 \\
28\end{array}$ \\
\hline \multirow[t]{3}{*}{ U.S. 321 Intersection w/ SR 416} & SR $416 \mathrm{SB}$ & 152 & $\begin{array}{l}\text { Right Turn } \\
\text { Left Tum }\end{array}$ & $\begin{array}{l}75.66 \% \\
24.34 \%\end{array}$ & $\begin{array}{r}115 \\
37\end{array}$ \\
\hline & U.S. 321 WB & 1363 & $\begin{array}{l}\text { Right Turn } \\
\text { Through }\end{array}$ & $\begin{array}{l}17.46 \% \\
82.54 \%\end{array}$ & $\begin{array}{r}238 \\
1125\end{array}$ \\
\hline & U.S. $321 \mathrm{~EB}$ & 548 & $\begin{array}{l}\text { Through } \\
\text { Left Tum }\end{array}$ & $\begin{array}{r}95.99 \% \\
4.01 \%\end{array}$ & $\begin{array}{r}526 \\
22\end{array}$ \\
\hline \multirow[t]{4}{*}{ SR 416 Intersection w/ Webb Creek Rd } & Webb Cr Rd WB & 122 & $\begin{array}{l}\text { Right Turn } \\
\text { Through } \\
\text { Left Tum }\end{array}$ & $\begin{array}{r}4.10 \% \\
44.26 \% \\
51.64 \%\end{array}$ & $\begin{array}{r}5 \\
54 \\
63\end{array}$ \\
\hline & SR $416 \mathrm{SB}$ & 163 & $\begin{array}{l}\text { Right Turn } \\
\text { Through } \\
\text { Left Tum }\end{array}$ & $\begin{array}{r}32.52 \% \\
66.87 \% \\
0.61 \%\end{array}$ & $\begin{array}{r}53 \\
109 \\
1\end{array}$ \\
\hline & SR $416 \mathrm{NB}$ & 275 & $\begin{array}{l}\text { Right Tum } \\
\text { Through } \\
\text { Left Tum }\end{array}$ & $\begin{array}{l}23.27 \% \\
29.82 \% \\
46.91 \%\end{array}$ & $\begin{array}{r}64 \\
82 \\
129\end{array}$ \\
\hline & FH Pkwy ramp & 23 & $\begin{array}{l}\text { Right Turn } \\
\text { Through } \\
\text { Left Tum }\end{array}$ & $\begin{array}{r}4.35 \% \\
47.83 \% \\
47.83 \%\end{array}$ & $\begin{array}{r}1 \\
11 \\
11\end{array}$ \\
\hline \multirow[t]{4}{*}{ SR 416 Intersection w/ Foothills Pkwy $8 \mathrm{~B} / 8 \mathrm{C}$} & FH Pkwy 8B WB & 130 & Through & $100.00 \%$ & 130 \\
\hline & FH Pkwy 8C EB & 240 & Through & $100.00 \%$ & 240 \\
\hline & SR $416 \mathrm{SB}$ & 64 & Through & $100.00 \%$ & 64 \\
\hline & SR $416 \mathrm{NB}$ & 116 & Through & $100.00 \%$ & 116 \\
\hline
\end{tabular}


Table K.17. Weekday A.M. peak-hour intersection volume projections for 2006 -interchange at Highway 416 (south ramp option)-8B not opened until $8 \mathrm{C}$ completed

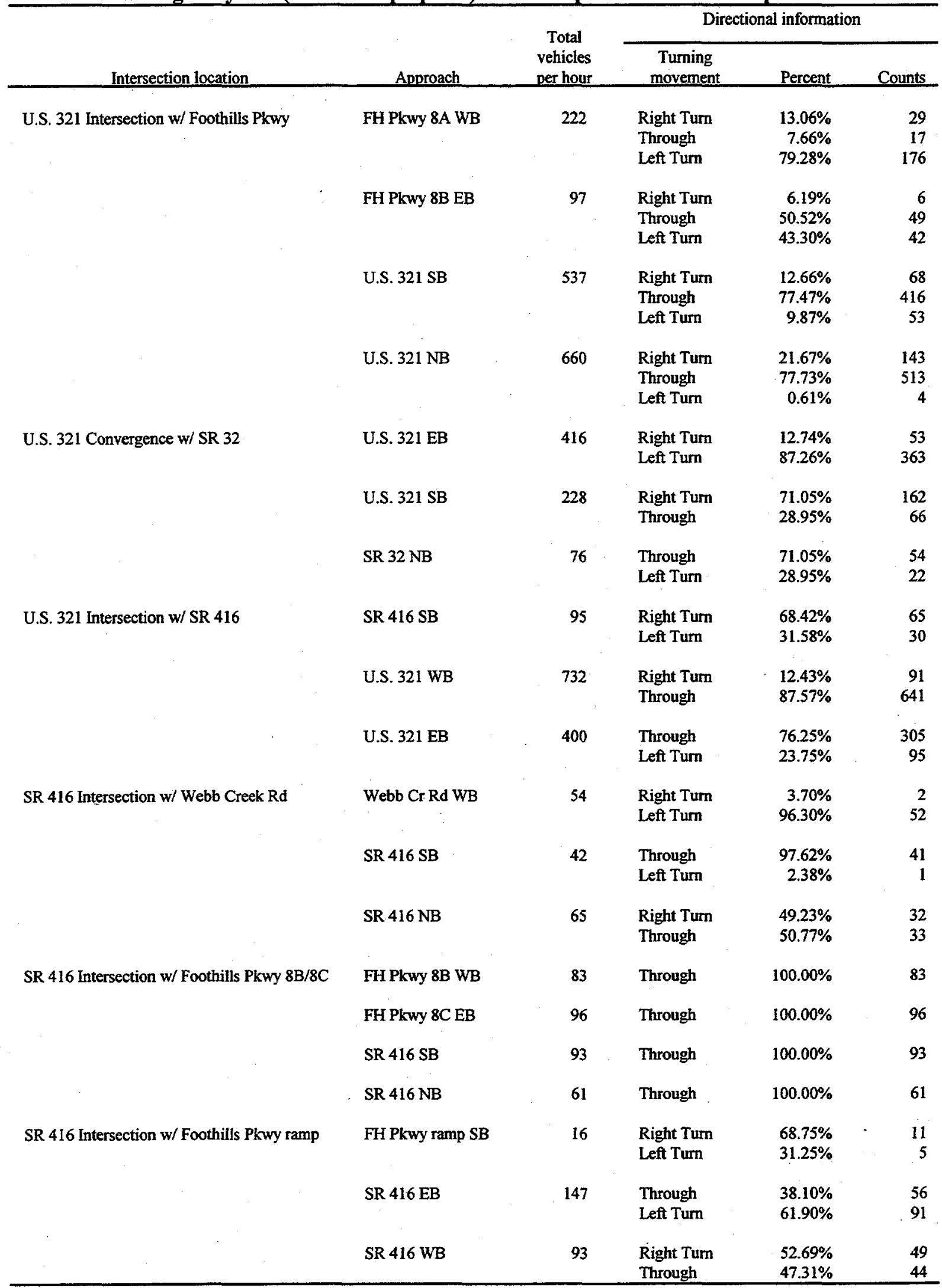


Table K.18. Weekday P.M. peak-hour intersection volume projections for 2006-interchange at Highway 416 (south ramp option)-8B not opened until $8 \mathrm{C}$ completed

\begin{tabular}{|c|c|c|c|c|c|}
\hline \multirow[b]{2}{*}{ Intersection location } & \multirow[b]{2}{*}{ Approach } & \multirow{2}{*}{$\begin{array}{c}\text { Total } \\
\text { vehicles } \\
\text { per hour }\end{array}$} & \multicolumn{3}{|c|}{ Directional information } \\
\hline & & & $\begin{array}{c}\text { Turning } \\
\text { movement }\end{array}$ & Percent & Counts \\
\hline \multirow[t]{6}{*}{ U.S. 321 Intersection w/ Foothills Pkwy } & FH Pkwy 8A WB & 163 & Right Tum & $15.95 \%$ & 26 \\
\hline & & & Through & $7.36 \%$ & 12 \\
\hline & & & Left Turn & $76.69 \%$ & 125 \\
\hline & FH Pkwy 8B EB & 128 & Right Tum & $6.25 \%$ & 8 \\
\hline & & & Through & $50.78 \%$ & 65 \\
\hline & & & Left Turn & $42.97 \%$ & 55 \\
\hline & U.S. $321 \mathrm{SB}$ & 555 & Right Turn & $13.69 \%$ & 76 \\
\hline & & & Through & $83.24 \%$ & 462 \\
\hline & & & Left Turn & $3.06 \%$ & 17 \\
\hline & U.S. $321 \mathrm{NB}$ & 671 & Right Turn & $14.31 \%$ & 96 \\
\hline & & & Through & $84.95 \%$ & 570 \\
\hline & & & Left Turn & $0.75 \%$ & 5 \\
\hline \multirow[t]{6}{*}{ U.S. 321 Convergence w/ SR 32} & U.S. $321 \mathrm{~EB}$ & 551 & Right Tum & $13.07 \%$ & 72 \\
\hline & & & Left Turn & $86.93 \%$ & 479 \\
\hline & U.S. 321 SB & 228 & Right Tum & $71.49 \%$ & 163 \\
\hline & & & Through & $28.51 \%$ & 65 \\
\hline & SR $32 \mathrm{NB}$ & 54 & Through & $68.52 \%$ & 37 \\
\hline & & & Left Tum & $31.48 \%$ & 17 \\
\hline \multirow[t]{6}{*}{ U.S. 321 Intersection w/ SR 416} & SR $416 \mathrm{SB}$ & 61 & Right Turn & $72.13 \%$ & 44 \\
\hline & & & Left Tum & $27.87 \%$ & 17 \\
\hline & U.S. $321 \mathrm{WB}$ & 804 & Right Tum & $24.75 \%$ & 199 \\
\hline & & & Through & $75.25 \%$ & 605 \\
\hline & U.S. $321 \mathrm{~EB}$ & 316 & Through & $83.54 \%$ & 264 \\
\hline & & & Left Turn & $16.46 \%$ & 52 \\
\hline \multirow[t]{6}{*}{ SR 416 Intersection w/ Webb Creek Rd } & Webb Cr Rd WB & 58 & Right Tum & $5.17 \%$ & 3 \\
\hline & & & Left Turn & $94.83 \%$ & 55 \\
\hline & SR $416 \mathrm{SB}$ & 53 & Through & $98.11 \%$ & 52 \\
\hline & & & Left Tum & $1.89 \%$ & 1 \\
\hline & SR 416 NB & 84 & Right Turn & $45.24 \%$ & 38 \\
\hline & & & Through & $54.76 \%$ & 46 \\
\hline \multirow[t]{4}{*}{ SR 416 Intersection w/ Foothills Pkwy 8B/8C } & FH Pkwy 8B WB & 84 & Through & $100.00 \%$ & 84 \\
\hline & FH Pkwy 8C EB & 126 & Through & $100.00 \%$ & 126 \\
\hline & SR $416 \mathrm{SB}$ & 107 & Through & $100.00 \%$ & 107 \\
\hline & SR $416 \mathrm{NB}$ & 80 & Through & $100.00 \%$ & 80 \\
\hline \multirow[t]{6}{*}{ SR 416 Intersection w/ Foothills Pkwy ramp } & FH Pkwy ramp SB & 21 & Right Turn & $66.67 \%$ & 14 \\
\hline & & & Left Turn & $33.33 \%$ & 7 \\
\hline & SR $416 \mathrm{~EB}$ & 191 & Through & $38.22 \%$ & 73 \\
\hline & & & Left Tum & $61.78 \%$ & 118 \\
\hline & SR 416 WB & 107 & Right Turn & $52.34 \%$ & 56 \\
\hline & & & Through & $47.66 \%$ & 51 \\
\hline
\end{tabular}


Table K.19. Weekend A.M. peak-hour intersection volume projections for 2006-interchange at Highway 416 (south ramp option)-8B not opened until $8 \mathrm{C}$ completed

\begin{tabular}{|c|c|c|c|c|c|}
\hline \multirow[b]{2}{*}{ Intersection location } & \multirow[b]{2}{*}{ Approach } & \multirow{2}{*}{$\begin{array}{c}\begin{array}{c}\text { Total } \\
\text { vehicles } \\
\text { per hour }\end{array} \\
\end{array}$} & \multicolumn{3}{|c|}{ Directional information } \\
\hline & & & $\begin{array}{c}\text { Turning } \\
\text { movement }\end{array}$ & Percent & Counts \\
\hline \multirow[t]{4}{*}{ U.S. 321 Intersection w/ Foothills Pkwy } & FH Pkwy 8A WB & 375 & $\begin{array}{l}\text { Right Turn } \\
\text { Through } \\
\text { Left Tum }\end{array}$ & $\begin{array}{r}13.07 \% \\
7.73 \% \\
79.20 \%\end{array}$ & $\begin{array}{r}49 \\
29 \\
297\end{array}$ \\
\hline & FH Pkwy 8B EB & 132 & $\begin{array}{l}\text { Right Turn } \\
\text { Through } \\
\text { Left Turn }\end{array}$ & $\begin{array}{r}6.06 \% \\
50.76 \% \\
43.18 \%\end{array}$ & $\begin{array}{r}8 \\
67 \\
57\end{array}$ \\
\hline & U.S. $321 \mathrm{SB}$ & 591 & $\begin{array}{l}\text { Right Tum } \\
\text { Through } \\
\text { Left Turn }\end{array}$ & $\begin{array}{r}12.69 \% \\
77.33 \% \\
9.98 \%\end{array}$ & $\begin{array}{r}75 \\
457 \\
59\end{array}$ \\
\hline & U.S. $321 \mathrm{NB}$ & 813 & $\begin{array}{l}\text { Right Tum } \\
\text { Through } \\
\text { Left Tum }\end{array}$ & $\begin{array}{r}21.77 \% \\
77.61 \% \\
0.62 \%\end{array}$ & $\begin{array}{r}177 \\
631 \\
5\end{array}$ \\
\hline \multirow[t]{3}{*}{ U.S. 321 Convergence w/ SR 32} & U.S. $321 \mathrm{~EB}$ & 570 & $\begin{array}{l}\text { Right Tum } \\
\text { Left Turn }\end{array}$ & $\begin{array}{l}12.81 \% \\
87.19 \%\end{array}$ & $\begin{array}{r}73 \\
497\end{array}$ \\
\hline & U.S. $321 \mathrm{SB}$ & 519 & $\begin{array}{l}\text { Right Turn } \\
\text { Through }\end{array}$ & $\begin{array}{l}70.91 \% \\
29.09 \%\end{array}$ & $\begin{array}{l}368 \\
151\end{array}$ \\
\hline & SR $32 \mathrm{NB}$ & 104 & $\begin{array}{l}\text { Through } \\
\text { Left Turn }\end{array}$ & $\begin{array}{l}72.12 \% \\
27.88 \%\end{array}$ & $\begin{array}{l}75 \\
29\end{array}$ \\
\hline \multirow[t]{3}{*}{ U.S. 321 Intersection w/ SR 416} & SR $416 \mathrm{SB}$ & 103 & $\begin{array}{l}\text { Right Turn } \\
\text { Left Tum }\end{array}$ & $\begin{array}{l}68.93 \% \\
31.07 \%\end{array}$ & $\begin{array}{l}71 \\
32\end{array}$ \\
\hline & U.S. $321 \mathrm{WB}$ & 893 & $\begin{array}{l}\text { Right Turn } \\
\text { Through }\end{array}$ & $\begin{array}{l}12.99 \% \\
87.01 \%\end{array}$ & $\begin{array}{l}116 \\
777\end{array}$ \\
\hline & U.S. $321 \mathrm{~EB}$ & 509 & $\begin{array}{l}\text { Through } \\
\text { Left Turn }\end{array}$ & $\begin{array}{l}76.03 \% \\
23.97 \%\end{array}$ & $\begin{array}{l}387 \\
122\end{array}$ \\
\hline \multirow[t]{3}{*}{ SR 416 Intersection w/ Webb Creek Rd } & Webb Cr Rd WB & 34 & $\begin{array}{l}\text { Right Turn } \\
\text { Left Tum }\end{array}$ & $\begin{array}{r}2.94 \% \\
97.06 \%\end{array}$ & $\begin{array}{r}1 \\
33\end{array}$ \\
\hline & SR 416 SB & 31 & $\begin{array}{l}\text { Through } \\
\text { Left Turn }\end{array}$ & $\begin{array}{r}96.77 \% \\
3.23 \%\end{array}$ & $\begin{array}{r}30 \\
1\end{array}$ \\
\hline & SR 416 NB & 54 & $\begin{array}{l}\text { Right Tum } \\
\text { Through }\end{array}$ & $\begin{array}{l}48.15 \% \\
51.85 \%\end{array}$ & $\begin{array}{l}26 \\
28\end{array}$ \\
\hline \multirow[t]{4}{*}{ SR 416 Intersection w/ Foothills Pkwy 8B/8C } & FH Pkwy 8B WB & 108 & Through & $100.00 \%$ & 108 \\
\hline & FH Pkwy 8C EB & 131 & Through & $100.00 \%$ & 131 \\
\hline & SR $416 \mathrm{SB}$. & 63 & Through & $100.00 \%$ & 63 \\
\hline & SR $416 \mathrm{NB}$ & 54 & Through & $100.00 \%$ & 54 \\
\hline \multirow[t]{3}{*}{ SR 416 Intersection w/ Foothills Pkwy ramp } & FH Pkwy ramp SB & 22 & $\begin{array}{l}\text { Right Turn } \\
\text { Left Tum }\end{array}$ & $\begin{array}{l}68.18 \% \\
31.82 \%\end{array}$ & $\begin{array}{r}15 \\
7\end{array}$ \\
\hline & SR 416 EB & 123 & $\begin{array}{l}\text { Through } \\
\text { Left Turn }\end{array}$ & $\begin{array}{l}38.21 \% \\
61.79 \%\end{array}$ & $\begin{array}{l}47 \\
76\end{array}$ \\
\hline & SR 416 WB & 63 & $\begin{array}{l}\text { Right Turn } \\
\text { Through }\end{array}$ & $\begin{array}{l}52.38 \% \\
47.62 \% \\
\end{array}$ & $\begin{array}{l}33 \\
30 \\
\end{array}$ \\
\hline
\end{tabular}


Table K.20. Weekend P.M. peak-hour intersection volume projections for 2006-interchange at Highway 416 (south ramp option)-8B not opened until $8 \mathrm{C}$ completed

\begin{tabular}{|c|c|c|c|c|c|}
\hline \multirow[b]{2}{*}{ Intersection location } & \multirow[b]{2}{*}{ Approach } & \multirow{2}{*}{$\begin{array}{c}\text { Total } \\
\text { vehicles } \\
\text { per hour }\end{array}$} & \multicolumn{3}{|c|}{ Directional information } \\
\hline & & & $\begin{array}{c}\text { Turning } \\
\text { movement }\end{array}$ & Percent & Counts \\
\hline \multirow[t]{4}{*}{ U.S. 321 Intersection w/ Foothills Pkwy } & FH Pkwy 8A WB & 229 & $\begin{array}{l}\text { Right Turn } \\
\text { Through } \\
\text { Left Turn }\end{array}$ & $\begin{array}{r}16.59 \% \\
7.42 \% \\
75.98 \%\end{array}$ & $\begin{array}{r}38 \\
17 \\
174\end{array}$ \\
\hline & FH Pkwy 8B EB & 153 & $\begin{array}{l}\text { Right Turn } \\
\text { Through } \\
\text { Left Turn }\end{array}$ & $\begin{array}{r}5.88 \% \\
50.98 \% \\
43.14 \%\end{array}$ & $\begin{array}{r}9 \\
78 \\
66\end{array}$ \\
\hline & U.S. $321 \mathrm{SB}$ & 651 & $\begin{array}{l}\text { Right Turn } \\
\text { Through } \\
\text { Left Tum }\end{array}$ & $\begin{array}{r}13.67 \% \\
83.26 \% \\
3.07 \%\end{array}$ & $\begin{array}{r}89 \\
542 \\
20\end{array}$ \\
\hline & U.S. $321 \mathrm{NB}$ & 766 & $\begin{array}{l}\text { Right Turn } \\
\text { Through } \\
\text { Left Tum }\end{array}$ & $\begin{array}{r}14.23 \% \\
85.12 \% \\
0.65 \%\end{array}$ & $\begin{array}{r}109 \\
652 \\
5\end{array}$ \\
\hline \multirow[t]{3}{*}{ U.S. 321 Convergence w/ SR 32} & U.S. $321 \mathrm{~EB}$ & 663 & $\begin{array}{l}\text { Right Turn } \\
\text { Left Tum }\end{array}$ & $\begin{array}{l}13.12 \% \\
86.88 \%\end{array}$ & $\begin{array}{r}87 \\
576\end{array}$ \\
\hline & U.S. $321 \mathrm{SB}$ & 432 & $\begin{array}{l}\text { Right Turn } \\
\text { Through }\end{array}$ & $\begin{array}{l}71.30 \% \\
28.70 \%\end{array}$ & $\begin{array}{l}308 \\
124\end{array}$ \\
\hline & SR $32 \mathrm{NB}$ & 69 & $\begin{array}{l}\text { Through } \\
\text { Left Turn }\end{array}$ & $\begin{array}{l}68.12 \% \\
31.88 \%\end{array}$ & $\begin{array}{l}47 \\
22\end{array}$ \\
\hline \multirow[t]{3}{*}{ U.S. 321 Intersection w/ SR 416} & SR $416 \mathrm{SB}$ & 119 & $\begin{array}{l}\text { Right Tum } \\
\text { Left Turn }\end{array}$ & $\begin{array}{l}71.43 \% \\
28.57 \%\end{array}$ & $\begin{array}{l}85 \\
34\end{array}$ \\
\hline & U.S. $321 \mathrm{WB}$ & 1074 & $\begin{array}{l}\text { Right Turn } \\
\text { Through }\end{array}$ & $\begin{array}{l}24.67 \% \\
75.33 \%\end{array}$ & $\begin{array}{l}265 \\
809\end{array}$ \\
\hline & U.S. $321 \mathrm{~EB}$ & 448 & $\begin{array}{l}\text { Through } \\
\text { Left Turn }\end{array}$ & $\begin{array}{l}83.93 \% \\
16.07 \%\end{array}$ & $\begin{array}{r}376 \\
72\end{array}$ \\
\hline \multirow[t]{3}{*}{ SR 416 Intersection w/ Webb Creek Rd } & Webb Cr Rd WB & 43 & $\begin{array}{l}\text { Right Turn } \\
\text { Left Tum }\end{array}$ & $\begin{array}{r}4.65 \% \\
95.35 \%\end{array}$ & $\begin{array}{r}2 \\
41\end{array}$ \\
\hline & SR $416 \mathrm{SB}$ & 72 & $\begin{array}{l}\text { Through } \\
\text { Left Turn }\end{array}$ & $\begin{array}{r}98.61 \% \\
1.39 \%\end{array}$ & $\begin{array}{r}71 \\
1\end{array}$ \\
\hline & SR $416 \mathrm{NB}$ & 84 & $\begin{array}{l}\text { Right Turn } \\
\text { Through }\end{array}$ & $\begin{array}{l}45.24 \% \\
54.76 \%\end{array}$ & $\begin{array}{l}38 \\
46\end{array}$ \\
\hline \multirow[t]{4}{*}{ SR 416 Intersection w/ Foothills Pkwy 8B/8C } & FH Pkwy 8B wB & 101 & Through & $100.00 \%$ & 101 \\
\hline & FH Pkwy 8C EB & 152 & Through & $100.00 \%$ & 152 \\
\hline & SR 416 SB & 112 & Through & $100.00 \%$ & 112 \\
\hline & SR 416 NB & 81 & Through & $100.00 \%$ & 81 \\
\hline \multirow[t]{3}{*}{ SR 416 Intersection w/ Foothills Pkwy ramp } & FH Pkwy ramp SB & 25 & $\begin{array}{l}\text { Right Turn } \\
\text { Left Turn }\end{array}$ & $\begin{array}{l}68.00 \% \\
32.00 \%\end{array}$ & $\begin{array}{r}17 \\
8\end{array}$ \\
\hline & SR $416 \mathrm{~EB}$ & 191 & $\begin{array}{l}\text { Through } \\
\text { Left Turn }\end{array}$ & $\begin{array}{l}38.22 \% \\
61.78 \%\end{array}$ & $\begin{array}{r}73 \\
118\end{array}$ \\
\hline & SR 416 WB & 112 & $\begin{array}{l}\text { Right Turn } \\
\text { Through }\end{array}$ & $\begin{array}{l}52.68 \% \\
47.32 \%\end{array}$ & $\begin{array}{l}59 \\
53\end{array}$ \\
\hline
\end{tabular}


Table K.21. Weekday A.M. peak-hour intersection volume projections for 2026-interchange at Highway 416 (south ramp option)-8B not opened until $8 \mathrm{C}$ completed

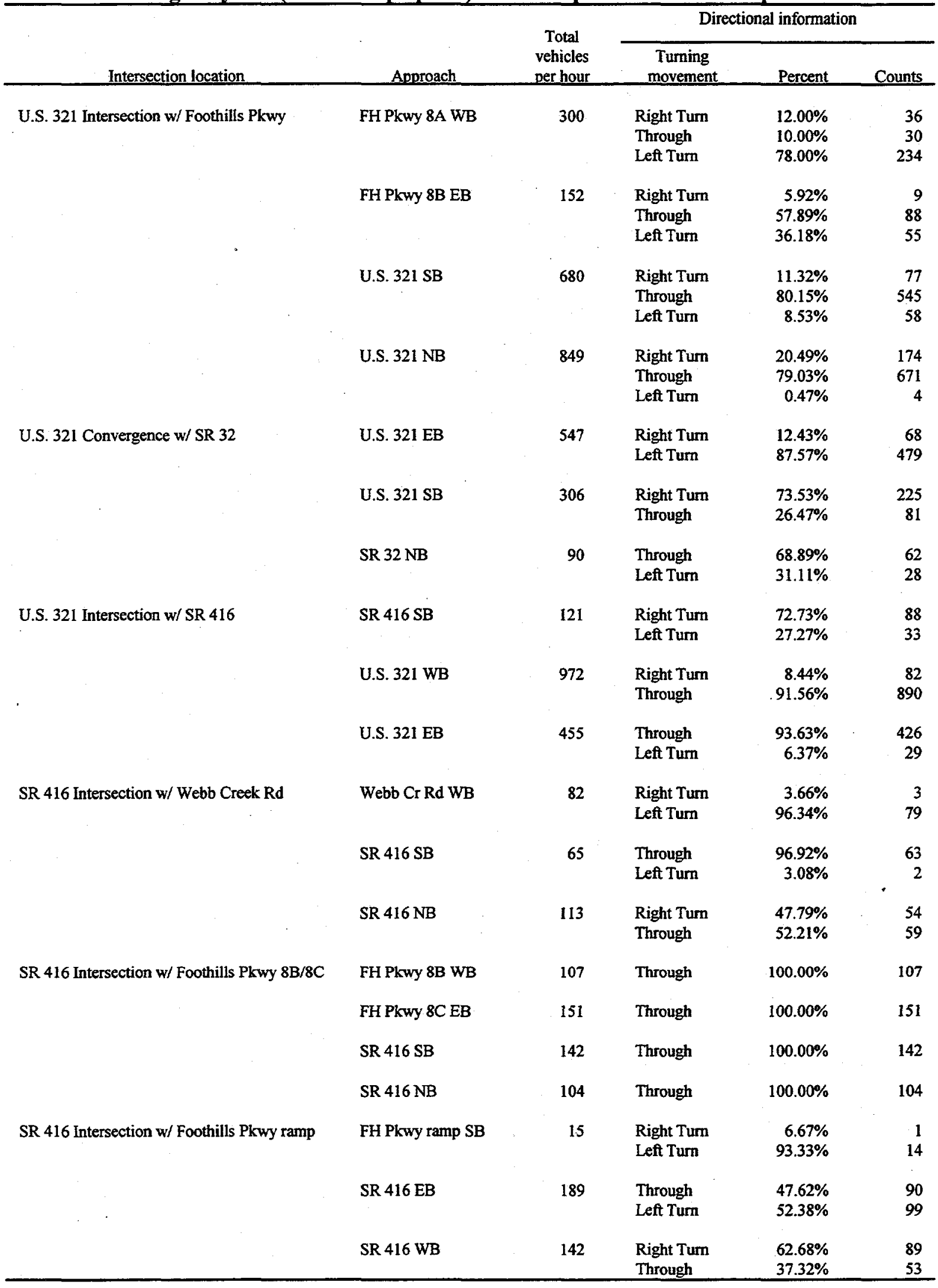


Table K.22. Weekday P.M. peak-hour intersection volume projections for 2026-interchange at Highway 416 (south ramp option)-8B not opened until 8C completed

\begin{tabular}{|c|c|c|c|c|c|}
\hline \multirow[b]{2}{*}{ Intersection location } & \multirow[b]{2}{*}{ Approach } & \multirow{2}{*}{$\begin{array}{c}\text { Total } \\
\text { vehicles } \\
\text { per hour }\end{array}$} & \multicolumn{3}{|c|}{ Directional information } \\
\hline & & & $\begin{array}{c}\text { Turning } \\
\text { movement }\end{array}$ & Percent & Counts \\
\hline \multirow[t]{12}{*}{ U.S. 321 Intersection w/ Foothills Pkwy } & FH Pkwy 8A WB & 221 & Right Turn & $14.93 \%$ & 33 \\
\hline & & & Through & $9.50 \%$ & 21 \\
\hline & & & Left Turn & $75.57 \%$ & 167 \\
\hline & FH Pkwy 8B EB & 201 & Right Turn & $5.97 \%$ & 12 \\
\hline & & & Through & $57.71 \%$ & 116 \\
\hline & & & Left Tum & $36.32 \%$ & 73 \\
\hline & U.S. $321 \mathrm{SB}$ & 711 & Right Turn & $12.10 \%$ & 86 \\
\hline & & & Through & $85.23 \%$ & 606 \\
\hline & & & Left Tum & $2.67 \%$ & 19 \\
\hline & U.S. $321 \mathrm{NB}$ & 867 & Right Tum & $13.49 \%$ & 117 \\
\hline & & & Through & $85.93 \%$ & 745 \\
\hline & & & Left Turn & $0.58 \%$ & 5 \\
\hline \multirow[t]{6}{*}{ U.S. 321 Convergence w/ SR 32} & U.S. $321 \mathrm{~EB}$ & 724 & Right Tum & $12.71 \%$ & 92 \\
\hline & & & Left Tum & $87.29 \%$ & 632 \\
\hline & U.S. $321 \mathrm{SB}$ & 306 & Right Turn & $74.18 \%$ & 227 \\
\hline & & & Through & $25.82 \%$ & 79 \\
\hline & SR $32 \mathrm{NB}$ & 63 & Through & $66.67 \%$ & 42 \\
\hline & & & Left Turn & $33.33 \%$ & 21 \\
\hline \multirow[t]{6}{*}{ U.S. 321 Intersection w/ SR 416} & SR 416 SB & 78 & Right Tum & $76.92 \%$ & 60 \\
\hline & & & Left Turn & $23.08 \%$ & 18 \\
\hline & U.S. 321 WB & 1019 & Right Tum & $17.47 \%$ & 178 \\
\hline & & & Through & $82.53 \%$ & 841 \\
\hline & U.S. $321 \mathrm{~EB}$ & 385 & Through & $95.84 \%$ & 369 \\
\hline & & & Left Turn & $4.16 \%$ & 16 \\
\hline \multirow[t]{6}{*}{ SR 416 Intersection w/ Webb Creek Rd } & Webb Cr Rd WB & 89 & Right Tum & $6.74 \%$ & 6 \\
\hline & & & Left Turn & $93.26 \%$ & 83 \\
\hline & SR $416 \mathrm{SB}$ & 80 & Through & $98.75 \%$ & 79 \\
\hline & & & Left Turn & $1.25 \%$ & 1 \\
\hline & $S \dot{S R} 416 \mathrm{NB}$ & 146 & Right Tum & $43.84 \%$ & 64 \\
\hline & & & Through & $56.16 \%$ & 82 \\
\hline \multirow[t]{4}{*}{ SR 416 Intersection w/ Foothills Pkwy 8B/8C } & FH Pkwy 8B WB & 108 & Through & $100.00 \%$ & 108 \\
\hline & FH Pkwy 8C EB & 200 & Through & $100.00 \%$ & 200 \\
\hline & SR 416 SB & 163 & Through & $100.00 \%$ & 163 \\
\hline & SR 416 NB & 134 & Through & $100.00 \%$ & 134 \\
\hline \multirow[t]{6}{*}{ SR 416 Intersection w/ Foothills Pkwy ramp } & FH Pkwy ramp SB & 19 & Right Tum & $5.26 \%$ & 1 \\
\hline & & & Left Turn & $94.74 \%$ & 18 \\
\hline & SR $416 \mathrm{~EB}$ & 245 & Through & $47.35 \%$ & 116 \\
\hline & & & Left Turn & $52.65 \%$ & 129 \\
\hline & SR 416 WB & 163 & Right Tum & $62.58 \%$ & 102 \\
\hline & & & Through & $37.42 \%$ & 61 \\
\hline
\end{tabular}


Table K.23. Weekend A.M. peak-hour intersection volume projections for 2026-interchange at Highway 416 (south ramp option) $-8 \mathrm{~B}$ not opened until $8 \mathrm{C}$ completed

\begin{tabular}{|c|c|c|c|c|c|}
\hline \multirow[b]{2}{*}{ Intersection location } & \multirow[b]{2}{*}{ Approach } & \multirow{2}{*}{$\begin{array}{c}\text { Total } \\
\text { vehicles } \\
\text { per hour }\end{array}$} & \multicolumn{3}{|c|}{ Directional information } \\
\hline & & & $\begin{array}{c}\text { Turning } \\
\text { movement }\end{array}$ & Percent & Counts \\
\hline \multirow[t]{4}{*}{ U.S. 321 Intersection w/ Foothills Pkwy } & FH Pkwy 8A WB & 507 & $\begin{array}{l}\text { Right Turn } \\
\text { Through } \\
\text { Left Turn }\end{array}$ & $\begin{array}{r}12.03 \% \\
9.86 \% \\
78.11 \%\end{array}$ & $\begin{array}{r}61 \\
50 \\
396\end{array}$ \\
\hline & FH Pkwy 8B EB & 208 & $\begin{array}{l}\text { Right Tum } \\
\text { Through } \\
\text { Left Tum }\end{array}$ & $\begin{array}{r}5.77 \% \\
57.69 \% \\
36.54 \%\end{array}$ & $\begin{array}{r}12 \\
120 \\
76\end{array}$ \\
\hline & U.S. $321 \mathrm{SB}$ & 749 & $\begin{array}{l}\text { Right Turn } \\
\text { Through } \\
\text { Left Turn }\end{array}$ & $\begin{array}{r}11.35 \% \\
79.97 \% \\
8.68 \%\end{array}$ & $\begin{array}{r}85 \\
599 \\
65\end{array}$ \\
\hline & U.S. $321 \mathrm{NB}$ & 1046 & $\begin{array}{l}\text { Right Tum } \\
\text { Through } \\
\text { Left Turn }\end{array}$ & $\begin{array}{r}20.65 \% \\
78.87 \% \\
0.48 \%\end{array}$ & $\begin{array}{r}216 \\
825 \\
5\end{array}$ \\
\hline \multirow[t]{3}{*}{ U.S. 321 Convergence w/ SR 32} & U.S. $321 \mathrm{~EB}$ & 749 & $\begin{array}{l}\text { Right Turn } \\
\text { Left Tum }\end{array}$ & $\begin{array}{l}12.42 \% \\
87.58 \%\end{array}$ & $\begin{array}{r}93 \\
656\end{array}$ \\
\hline & U.S. $321 \mathrm{SB}$ & 696 & $\begin{array}{l}\text { Right Turn } \\
\text { Through }\end{array}$ & $\begin{array}{l}73.56 \% \\
26.44 \%\end{array}$ & $\begin{array}{l}512 \\
184\end{array}$ \\
\hline & SR 32 NB & 123 & $\begin{array}{l}\text { Through } \\
\text { Left Turn }\end{array}$ & $\begin{array}{l}69.92 \% \\
30.08 \%\end{array}$ & $\begin{array}{l}86 \\
37\end{array}$ \\
\hline \multirow[t]{3}{*}{ U.S. 321 Intersection w/ SR 416} & SR 416 SB & 131 & $\begin{array}{l}\text { Right Turn } \\
\text { Left Turn }\end{array}$ & $\begin{array}{l}73.28 \% \\
26.72 \%\end{array}$ & $\begin{array}{l}96 \\
35\end{array}$ \\
\hline & U.S. $321 \mathrm{WB}$ & 1184 & $\begin{array}{l}\text { Right Turn } \\
\text { Through }\end{array}$ & $\begin{array}{r}8.78 \% \\
91.22 \%\end{array}$ & $\begin{array}{r}104 \\
1080\end{array}$ \\
\hline & U.S. $321 \mathrm{~EB}$ & 578 & $\begin{array}{l}\text { Through } \\
\text { Left Turn }\end{array}$ & $\begin{array}{r}93.60 \% \\
6.40 \%\end{array}$ & $\begin{array}{r}541 \\
37\end{array}$ \\
\hline \multirow[t]{3}{*}{ SR 416 lntersection w/ Webb Creek Rd } & Webb Cr Rd WB & 52 & $\begin{array}{l}\text { Right Turn } \\
\text { Left Turn }\end{array}$ & $\begin{array}{r}3.85 \% \\
96.15 \%\end{array}$ & $\begin{array}{r}2 \\
50\end{array}$ \\
\hline & SR 416 SB & 48 & $\begin{array}{l}\text { Through } \\
\text { Left Turn }\end{array}$ & $\begin{array}{r}95.83 \% \\
4.17 \%\end{array}$ & $\begin{array}{r}46 \\
2\end{array}$ \\
\hline & SR 416 NB & 94 & $\begin{array}{l}\text { Right Turn } \\
\text { Through }\end{array}$ & $\begin{array}{l}46.81 \% \\
53.19 \%\end{array}$ & $\begin{array}{l}44 \\
50\end{array}$ \\
\hline \multirow[t]{4}{*}{ SR 416 Intersection w/ Foothills Pkwy 8B/8C } & FH Pkwy 8B WB & 139 & Through & $100.00 \%$ & 139 \\
\hline & FH Pkwy 8C EB & 207 & Through & $100.00 \%$ & 207 \\
\hline & SR 416 SB & 96 & Through & $100.00 \%$ & 96 \\
\hline & SR $416 \mathrm{NB}$ & 94 & Through & $100.00 \%$ & 94 \\
\hline \multirow[t]{3}{*}{ SR 416 Intersection w/ Foothills Pkwy ramp } & FH Pkwy ramp SB & 20 & $\begin{array}{l}\text { Right Tum } \\
\text { Left Turn }\end{array}$ & $\begin{array}{r}5.00 \% \\
95.00 \%\end{array}$ & $\begin{array}{r}1 \\
19\end{array}$ \\
\hline & SR $416 \mathrm{~EB}$ & 158 & $\begin{array}{l}\text { Through } \\
\text { Left Tum }\end{array}$ & $\begin{array}{r}47.47 \% \\
52.53 \%\end{array}$ & $\begin{array}{l}75 \\
83\end{array}$ \\
\hline & SR 416 WB & 96 & $\begin{array}{l}\text { Right Turn } \\
\text { Through }\end{array}$ & $\begin{array}{l}62.50 \% \\
37.50 \%\end{array}$ & $\begin{array}{l}60 \\
36\end{array}$ \\
\hline
\end{tabular}


Table K.24. Weekend P.M. peak-hour intersection volume projections for 2026-interchange at Highway 416 (south ramp option) $-8 B$ not opened until $8 \mathrm{C}$ completed

\begin{tabular}{|c|c|c|c|c|c|}
\hline \multirow[b]{2}{*}{ Intersection location } & \multirow[b]{2}{*}{ Approach } & \multirow{2}{*}{$\begin{array}{c}\text { Total } \\
\text { vehicles } \\
\text { per hour }\end{array}$} & \multicolumn{3}{|c|}{ Directional information } \\
\hline & & & $\begin{array}{c}\text { Turning } \\
\text { movement }\end{array}$ & Percent & Counts \\
\hline \multirow[t]{4}{*}{ U.S. 321 Intersection w/ Foothills Pkwy } & FH Pkwy 8A WB & 308 & $\begin{array}{l}\text { Right Turn } \\
\text { Through } \\
\text { Left Turn }\end{array}$ & $\begin{array}{r}15.26 \% \\
9.42 \% \\
75.32 \%\end{array}$ & $\begin{array}{r}47 \\
29 \\
232\end{array}$ \\
\hline & FH Pkwy 8B EB & 241 & $\begin{array}{l}\text { Right Turn } \\
\text { Through } \\
\text { Left Tum }\end{array}$ & $\begin{array}{r}5.81 \% \\
57.68 \% \\
36.51 \%\end{array}$ & $\begin{array}{r}14 \\
139 \\
88\end{array}$ \\
\hline & U.S. $321 \mathrm{SB}$ & 833 & $\begin{array}{l}\text { Right Turn } \\
\text { Through } \\
\text { Left Turn }\end{array}$ & $\begin{array}{r}12.12 \% \\
85.23 \% \\
2.64 \%\end{array}$ & $\begin{array}{r}101 \\
710 \\
22\end{array}$ \\
\hline & U.S. $321 \mathrm{NB}$ & 991 & $\begin{array}{l}\text { Right Turn } \\
\text { Through } \\
\text { Left Tum }\end{array}$ & $\begin{array}{r}13.42 \% \\
86.07 \% \\
0.50 \%\end{array}$ & $\begin{array}{r}133 \\
853 \\
5\end{array}$ \\
\hline \multirow[t]{3}{*}{ U.S. 321 Convergence w/ SR 32} & U.S. 321 EB & 871 & $\begin{array}{l}\text { Right Turn } \\
\text { Left Turn }\end{array}$ & $\begin{array}{l}12.74 \% \\
87.26 \%\end{array}$ & $\begin{array}{l}111 \\
760\end{array}$ \\
\hline & U.S. $321 \mathrm{SB}$ & 580 & $\begin{array}{l}\text { Right Tum } \\
\text { Through }\end{array}$ & $\begin{array}{l}73.97 \% \\
26.03 \%\end{array}$ & $\begin{array}{l}429 \\
151\end{array}$ \\
\hline & SR $32 \mathrm{NB}$ & 82 & $\begin{array}{l}\text { Through } \\
\text { Left Turn }\end{array}$ & $\begin{array}{l}65.85 \% \\
34.15 \%\end{array}$ & $\begin{array}{l}54 \\
28\end{array}$ \\
\hline \multirow[t]{3}{*}{ U.S. 321 Intersection w/ SR 416} & SR 416 SB & 152 & $\begin{array}{l}\text { Right Turn } \\
\text { Left Turn }\end{array}$ & $\begin{array}{l}75.66 \% \\
24.34 \%\end{array}$ & $\begin{array}{r}115 \\
37\end{array}$ \\
\hline & U.S. 321 WB & 1363 & $\begin{array}{l}\text { Right Tum } \\
\text { Through }\end{array}$ & $\begin{array}{l}17.46 \% \\
82.54 \%\end{array}$ & $\begin{array}{r}238 \\
1125\end{array}$ \\
\hline & U.S. $321 \mathrm{~EB}$ & 548 & $\begin{array}{l}\text { Through } \\
\text { Left Turn }\end{array}$ & $\begin{array}{r}95.99 \% \\
4.01 \%\end{array}$ & $\begin{array}{r}526 \\
22\end{array}$ \\
\hline \multirow[t]{3}{*}{ SR 416 Intersection w/ Webb Creek Rd } & Webb Cr Rd WB & 68 & $\begin{array}{l}\text { Right Tum } \\
\text { Left Tum }\end{array}$ & $\begin{array}{r}7.35 \% \\
92.65 \%\end{array}$ & $\begin{array}{r}5 \\
63\end{array}$ \\
\hline & SR 416 SB & 110 & $\begin{array}{l}\text { Through } \\
\text { Left Turn }\end{array}$ & $\begin{array}{r}99.09 \% \\
0.91 \%\end{array}$ & $\begin{array}{r}109 \\
1\end{array}$ \\
\hline & SR $416 \mathrm{NB}$ & 146 & $\begin{array}{l}\text { Right Turn } \\
\text { Through }\end{array}$ & $\begin{array}{l}43.84 \% \\
56.16 \%\end{array}$ & $\begin{array}{l}64 \\
82\end{array}$ \\
\hline \multirow[t]{4}{*}{ SR 416 Intersection w/ Foothills Pkwy 8B/8C } & FH Pkwy 8B wB & 130 & Through & $100.00 \%$ & 130 \\
\hline & FH Pkwy 8C EB & 240 & Through & $100.00 \%$ & 240 \\
\hline & SR $416 \mathrm{SB}$ & 171 & Through & $100.00 \%$ & 171 \\
\hline & SR 416 NB & 138 & Through & $100.00 \%$ & 138 \\
\hline \multirow[t]{3}{*}{ SR 416 Intersection w/ Foothilis Pkwy ramp } & FH Pkwy ramp SB & 23 & $\begin{array}{l}\text { Right Turn } \\
\text { Left Turn }\end{array}$ & $\begin{array}{r}4.35 \% \\
95.65 \%\end{array}$ & $\begin{array}{r}1 \\
22\end{array}$ \\
\hline & SR $416 \mathrm{~EB}$ & 245 & $\begin{array}{l}\text { Through } \\
\text { Left Tum }\end{array}$ & $\begin{array}{l}47.35 \% \\
52.65 \%\end{array}$ & $\begin{array}{l}116 \\
129\end{array}$ \\
\hline & SR 416 WB & 171 & $\begin{array}{l}\text { Right Turn } \\
\text { Through }\end{array}$ & $\begin{array}{l}62.57 \% \\
37.43 \%\end{array}$ & $\begin{array}{r}107 \\
64 \\
\end{array}$ \\
\hline
\end{tabular}


Table K.25. Weekday A.M. peak-hour intersection volume projections for 2006-interchange at U.S. 321 (both options)-8B not opened until $8 \mathrm{C}$ completed

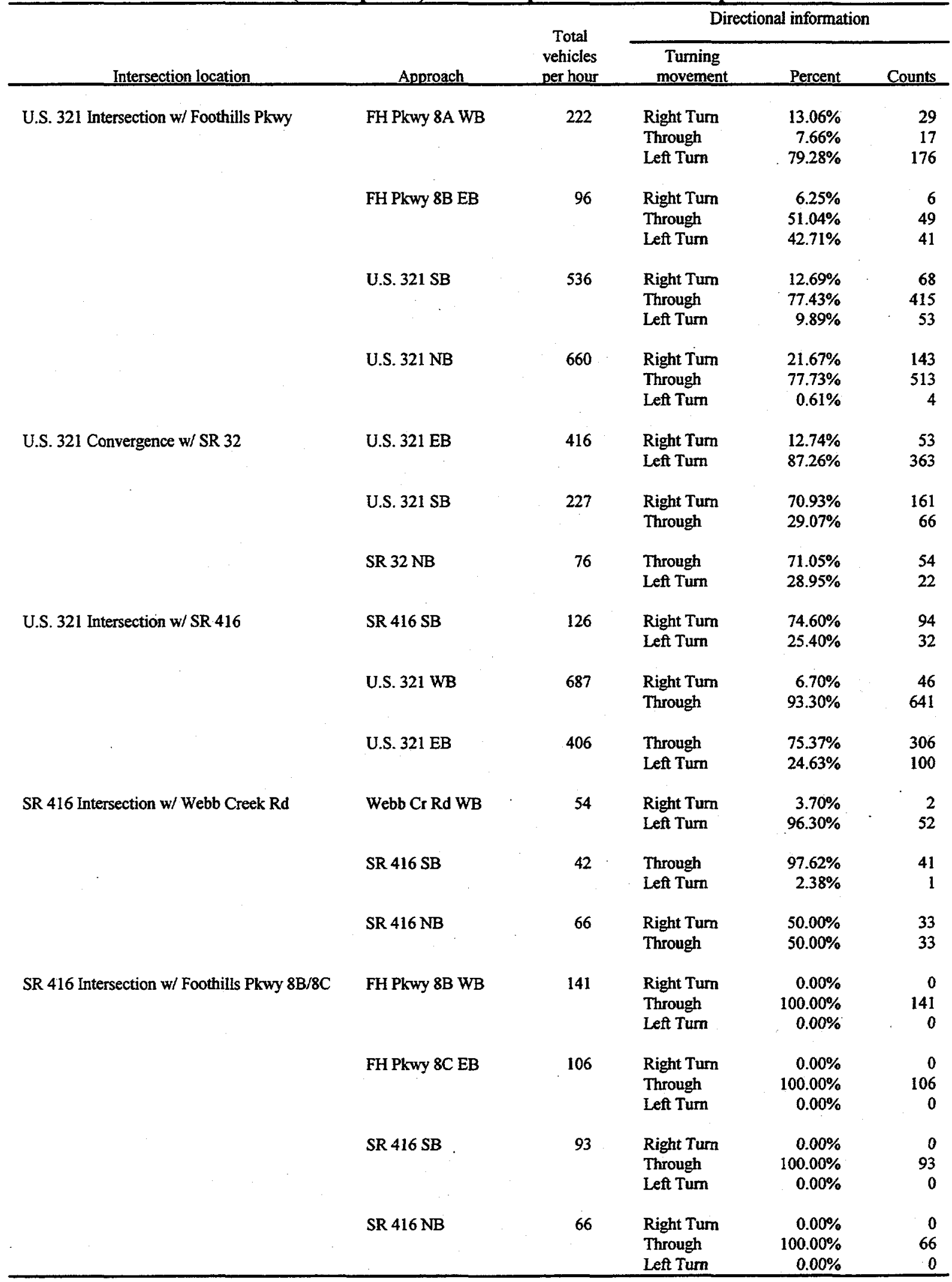


Table K.25. Weekday A.M. peak-hour intersection volume projections for 2006-interchange at U.S. 321 (directly connected)-8B not opened until 8C completed (continued)

\begin{tabular}{|c|c|c|c|c|c|}
\hline \multirow[b]{2}{*}{ Intersection location } & \multirow[b]{2}{*}{ Approach } & \multirow{2}{*}{$\begin{array}{c}\text { Total } \\
\text { vehicles } \\
\text { per hour }\end{array}$} & \multicolumn{3}{|c|}{ Directional information } \\
\hline & & & $\begin{array}{c}\text { Turning } \\
\text { movement }\end{array}$ & Percent & Counts \\
\hline \multirow[t]{3}{*}{ U.S. 321 Intersection w/ Foothills Pkwy 8B } & FH Pkwy 8B & 12 & $\begin{array}{l}\text { Right Turn } \\
\text { Left Tum }\end{array}$ & $\begin{array}{r}100.00 \% \\
0.00 \%\end{array}$ & $\begin{array}{r}12 \\
0\end{array}$ \\
\hline & U.S. 321 WB & 728 & $\begin{array}{l}\text { Right Turn } \\
\text { Through }\end{array}$ & $\begin{array}{r}7.28 \% \\
92.72 \%\end{array}$ & $\begin{array}{r}53 \\
675\end{array}$ \\
\hline & U.S. $321 \mathrm{~EB}$ & 332 & $\begin{array}{l}\text { Through } \\
\text { Left Turn }\end{array}$ & $\begin{array}{r}99.40 \% \\
0.60 \%\end{array}$ & $\begin{array}{r}330 \\
2\end{array}$ \\
\hline
\end{tabular}

Table K.25. Weekday A.M. peak-hour intersection volume projections for 2006-interchange at U.S. 321 (Webb Creek Rd. connection)-8B not opened until 8C completed (continued)

\begin{tabular}{|c|c|c|c|c|c|}
\hline \multirow[b]{2}{*}{ Intersection location } & \multirow[b]{2}{*}{ Approach } & \multirow{2}{*}{$\begin{array}{c}\text { Total } \\
\text { vehicles } \\
\text { per hour }\end{array}$} & \multicolumn{3}{|c|}{ Directional information } \\
\hline & & & $\begin{array}{c}\text { Turning } \\
\text { movement }\end{array}$ & Percent & Counts \\
\hline \multirow[t]{3}{*}{ Foothills Pkwy 8B/Webb Creek Rd to US 321} & FH Pkwy 8B & 56 & $\begin{array}{l}\text { Right Turn } \\
\text { Left Tum }\end{array}$ & $\begin{array}{l}60.71 \% \\
39.29 \%\end{array}$ & $\begin{array}{l}34 \\
22\end{array}$ \\
\hline & U.S. 321 WB & 750 & $\begin{array}{l}\text { Right Turn } \\
\text { Through }\end{array}$ & $\begin{array}{l}10.00 \% \\
90.00 \%\end{array}$ & $\begin{array}{r}75 \\
675\end{array}$ \\
\hline & U.S. $321 \mathrm{~EB}$ & 354 & $\begin{array}{l}\text { Through } \\
\text { Left Turn }\end{array}$ & $\begin{array}{r}93.22 \% \\
6.78 \%\end{array}$ & $\begin{array}{r}330 \\
24\end{array}$ \\
\hline
\end{tabular}


Table K.26. Weekday P.M. peak-hour intersection volume projections for 2006-interchange at U.S. 321 (both options) 8 B not opened until 8C completed

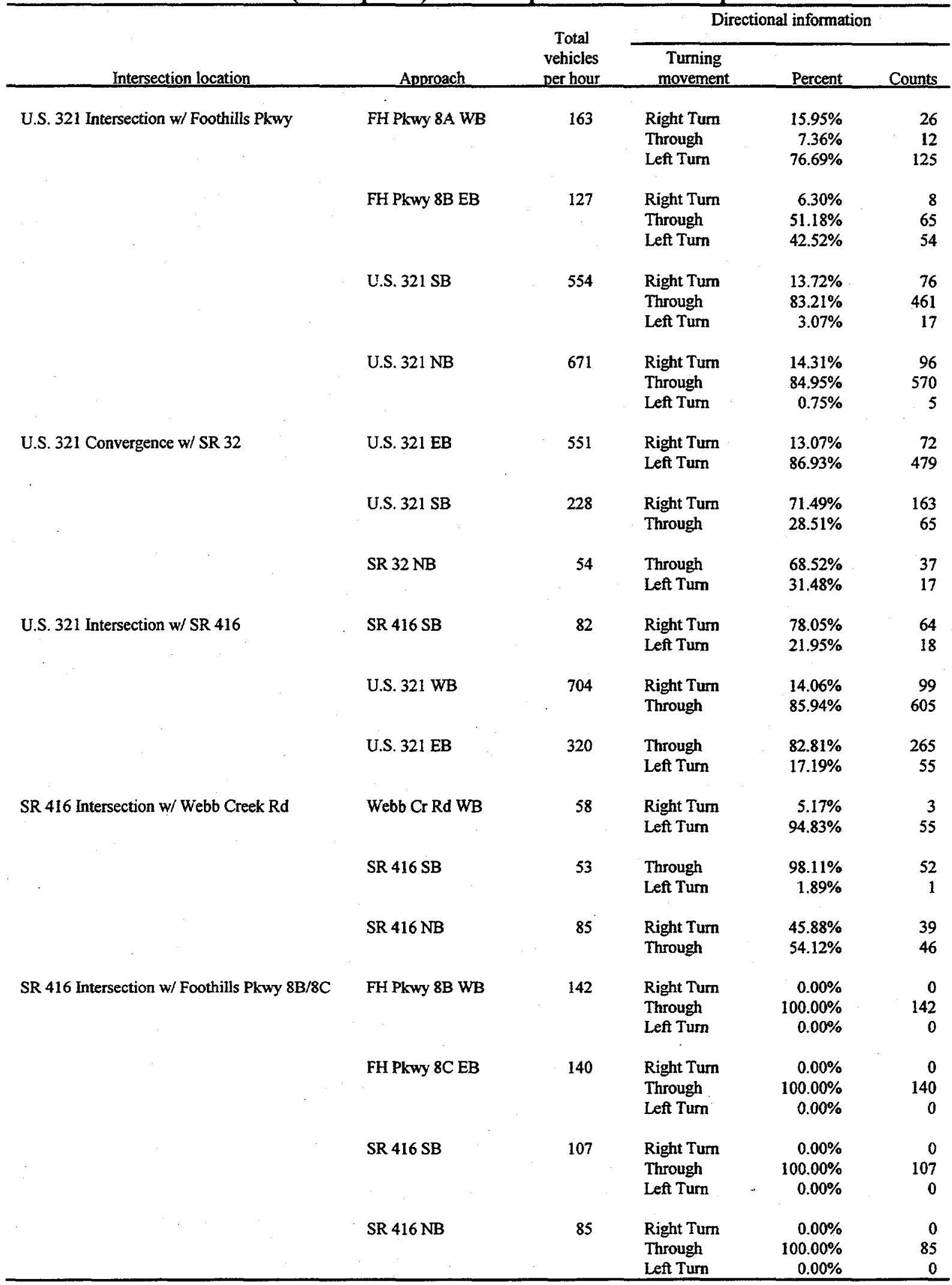


Table K.26. Weekday P.M. peak-hour intersection volume projections for 2006 -interchange at U.S. 321 (directly connected) $-8 B$ not opened until $8 \mathrm{C}$ completed (continued)

\begin{tabular}{|c|c|c|c|c|c|}
\hline \multirow[b]{2}{*}{ Intersection location } & \multirow[b]{2}{*}{ Approach } & \multirow{2}{*}{$\begin{array}{c}\text { Total } \\
\text { vehicles } \\
\text { per hour }\end{array}$} & \multicolumn{3}{|c|}{ Directional information } \\
\hline & & & $\begin{array}{c}\text { Turning } \\
\text { movement }\end{array}$ & Percent & Counts \\
\hline \multirow[t]{3}{*}{ U.S. 321 Intersection w/ Foothills Pkwy 8B } & FH Pkwy 8B & 15 & $\begin{array}{l}\text { Right Turn } \\
\text { Left Tum }\end{array}$ & $\begin{array}{r}100.00 \% \\
0.00 \%\end{array}$ & $\begin{array}{r}15 \\
0\end{array}$ \\
\hline & U.S. $321 \mathrm{WB}$ & 708 & $\begin{array}{l}\text { Right Turn } \\
\text { Through }\end{array}$ & $\begin{array}{r}9.89 \% \\
90.11 \%\end{array}$ & $\begin{array}{r}70 \\
638\end{array}$ \\
\hline & U.S. $321 \mathrm{~EB}$ & 289 & $\begin{array}{l}\text { Through } \\
\text { Left Turn }\end{array}$ & $\begin{array}{r}98.96 \% \\
1.04 \%\end{array}$ & $\begin{array}{r}286 \\
3\end{array}$ \\
\hline
\end{tabular}

Table K.26. Weekday P.M. peak-hour intersection volume projections for 2006-interchange at U.S. 321 (Webb Creek Rd. connection)-8B not opened until 8C completed (continued)

\begin{tabular}{|c|c|c|c|c|c|}
\hline \multirow[b]{2}{*}{ Intersection location } & \multirow[b]{2}{*}{ Approach } & \multirow{2}{*}{$\begin{array}{c}\text { Total } \\
\text { vehicles } \\
\text { per hour }\end{array}$} & \multicolumn{3}{|c|}{ Directional information } \\
\hline & & & $\begin{array}{c}\text { Turning } \\
\text { movement }\end{array}$ & Percent & Counts \\
\hline \multirow[t]{3}{*}{ Foothills Pkwy 8B/Webb Creek Rd to US 321} & FH Pkwy 8B & 65 & $\begin{array}{l}\text { Right Turn } \\
\text { Left Turn }\end{array}$ & $\begin{array}{l}61.54 \% \\
38.46 \%\end{array}$ & $\begin{array}{l}40 \\
25\end{array}$ \\
\hline & U.S. 321 WB & 733 & $\begin{array}{l}\text { Right Turn } \\
\text { Through }\end{array}$ & $\begin{array}{l}12.96 \% \\
87.04 \%\end{array}$ & $\begin{array}{r}95 \\
638\end{array}$ \\
\hline & U.S. $321 \mathrm{~EB}$ & 314 & $\begin{array}{l}\text { Through } \\
\text { Left Turn }\end{array}$ & $\begin{array}{r}91.08 \% \\
8.92 \%\end{array}$ & $\begin{array}{r}286 \\
28\end{array}$ \\
\hline
\end{tabular}


Table K.27. Weekend A.M. peak-hour intersection volume projections for 2006-interchange at U.S. 321 (both options)-8B not opened until 8C completed

\begin{tabular}{|c|c|c|c|c|c|}
\hline \multirow{2}{*}{ Intersection location } & \multirow[b]{2}{*}{ Approach } & \multirow{2}{*}{$\begin{array}{l}\text { Total } \\
\text { vehicles } \\
\text { per hour }\end{array}$} & \multicolumn{3}{|c|}{ Directional information } \\
\hline & & & $\begin{array}{c}\text { Turning } \\
\text { movement }\end{array}$ & Percent & Counts \\
\hline \multirow[t]{12}{*}{ U.S. 321 Intersection w/ Foothills Pkwy } & FH Pkwy 8A WB & 375 & Right Tum & $13.07 \%$ & 49 \\
\hline & & & Through & $7.73 \%$ & 29 \\
\hline & & & Left Turn & $79.20 \%$ & 297 \\
\hline & FH Pkwy 8B EB & 131 & Right Tum & $6.11 \%$ & 8 \\
\hline & & & Through & $51.15 \%$ & 67 \\
\hline & & & Left Turn & $42.75 \%$ & 56 \\
\hline & U.S. $321 \mathrm{SB}$ & $590^{\circ}$ & Right Turn & $12.71 \%$ & 75 \\
\hline & & & Through & $77.29 \%$ & 456 \\
\hline & & & Left Turn & $10.00 \%$ & 59 \\
\hline & U.S. $321 \mathrm{NB}$ & 813 & Right Tum & $21.77 \%$ & 177 \\
\hline & & & Through & $77.61 \%$ & 631 \\
\hline & & & Left Turn & $0.62 \%$ & 5 \\
\hline \multirow[t]{6}{*}{ U.S. 321 Convergence w/ SR 32} & U.S. $321 \mathrm{~EB}$ & 570 & Right Turn & $12.81 \%$ & 73 \\
\hline & & & Left Tum & $87.19 \%$ & 497 \\
\hline & U.S. $321 \mathrm{SB}$ & 518 & Right Turn & $70.85 \%$ & 367 \\
\hline & & & Through & $29.15 \%$ & 151 \\
\hline & SR 32 NB & 104 & Through & $72.12 \%$ & 75 \\
\hline & & & Left Tum & $27.88 \%$ & 29 \\
\hline \multirow[t]{6}{*}{ U.S. 321 Intersection w/ SR 416} & SR 416 SB & 136 & Right Tum & $75.00 \%$ & 102 \\
\hline & & & Left Turn & $25.00 \%$ & 34 \\
\hline & U.S. 321 WB & 835 & Right Tum & $6.95 \%$ & 58 \\
\hline & & & Through & $93.05 \%$ & 777 \\
\hline & U.S. $321 \mathrm{~EB}$ & 516 & Through & $75.19 \%$ & 388 \\
\hline & & & Left Tum & $24.81 \%$ & 128 \\
\hline \multirow[t]{6}{*}{ SR 416 Intersection w/ Webb Creek Rd } & Webb Cr Rd WB & 34 & Right Turn & $2.94 \%$ & 1 \\
\hline & & & Left Tum & $97.06 \%$ & 33 \\
\hline & SR 416 SB & 31 & Through & $96.77 \%$ & 30 \\
\hline & & & Left Tum & $3.23 \%$ & 1 \\
\hline & SR 416 NB & 55 & Right Turn & $49.09 \%$ & 27 \\
\hline & & & Through & $50.91 \%$ & 28 \\
\hline \multirow[t]{11}{*}{ SR 416 Intersection w/ Foothills Pkwy $8 \mathrm{~B} / 8 \mathrm{C}$} & FH Pkwy 8B WB & 183 & Right Turn & $0.00 \%$ & \\
\hline & & & Through & $100.00 \%$ & 183 \\
\hline & & & Left Turn & $0.00 \%$ & \\
\hline & FH Pkwy 8C EB & 145 & Right Tum & $0.00 \%$ & \\
\hline & & & $\begin{array}{l}\text { Through } \\
\text { Left Turn }\end{array}$ & $\begin{array}{r}100.00 \% \\
0.00 \%\end{array}$ & 145 \\
\hline & SR $416 \mathrm{SB}$ & 63 & Right Tum & $0.00 \%$ & \\
\hline & & & Through & $100.00 \%$ & 63 \\
\hline & & & Left Turn & $0.00 \%$ & \\
\hline & SR $416 \mathrm{NB}$ & 55 & Right Turn & $0.00 \%$ & \\
\hline & & & Through & $100.00 \%$ & 55 \\
\hline & & & Left Turn & $0.00 \%$ & \\
\hline
\end{tabular}


Table K.27. Weekend A.M. peak-hour intersection volume projections for 2006 -interchange at U.S. 321 (directly connected)-8B not opened until 8C completed (continued)

\begin{tabular}{|c|c|c|c|c|c|}
\hline \multirow[b]{2}{*}{ Intersection location } & \multirow[b]{2}{*}{ Approach } & \multirow{2}{*}{$\begin{array}{c}\text { Total } \\
\text { vehicles } \\
\text { per hour }\end{array}$} & \multicolumn{3}{|c|}{ Directional information } \\
\hline & & & $\begin{array}{c}\text { Turning } \\
\text { movement }\end{array}$ & Percent & Counts \\
\hline \multirow[t]{3}{*}{ U.S. 321 Intersection w/ Foothills Pkwy 8B } & FH Pkwy 8B & 16 & $\begin{array}{l}\text { Right Turn } \\
\text { Left Tum }\end{array}$ & $\begin{array}{r}100.00 \% \\
0.00 \%\end{array}$ & $\begin{array}{r}16 \\
0\end{array}$ \\
\hline & U.S. 321 WB & 892 & $\begin{array}{l}\text { Right Turn } \\
\text { Through }\end{array}$ & $\begin{array}{r}8.18 \% \\
91.82 \%\end{array}$ & $\begin{array}{r}73 \\
819\end{array}$ \\
\hline & U.S. $321 \mathrm{~EB}$ & 547 & $\begin{array}{l}\text { Through } \\
\text { Left Turn }\end{array}$ & $\begin{array}{l}76.60 \% \\
23.40 \%\end{array}$ & $\begin{array}{r}419 \\
3\end{array}$ \\
\hline
\end{tabular}

Table K.27. Weekend A.M. peak-hour intersection volume projections for 2006-interchange at U.S. 321 (Webb Creek Rd. connection)-8B not opened until 8C completed (continued)

\begin{tabular}{|c|c|c|c|c|c|}
\hline \multirow[b]{2}{*}{ Intersection location } & \multirow[b]{2}{*}{ Approach } & \multirow{2}{*}{$\begin{array}{c}\text { Total } \\
\text { vehicles } \\
\text { per hour }\end{array}$} & \multicolumn{3}{|c|}{ Directional information } \\
\hline & & & $\begin{array}{c}\text { Turning } \\
\text { movement }\end{array}$ & Percent & Counts \\
\hline \multirow[t]{3}{*}{ Foothills Pkwy 8B/Webb Creek Rd to US 321} & FH Pkwy 8B & 46 & $\begin{array}{l}\text { Right Tum } \\
\text { Left Tum }\end{array}$ & $\begin{array}{l}67.39 \% \\
32.61 \%\end{array}$ & $\begin{array}{l}31 \\
15\end{array}$ \\
\hline & U.S. 321 WB & 907 & $\begin{array}{l}\text { Right Turn } \\
\text { Through }\end{array}$ & $\begin{array}{r}9.70 \% \\
90.30 \%\end{array}$ & $\begin{array}{r}88 \\
819\end{array}$ \\
\hline & U.S. $321 \mathrm{~EB}$ & 547 & $\begin{array}{l}\text { Through } \\
\text { Left Tum }\end{array}$ & $\begin{array}{l}76.60 \% \\
23.40 \%\end{array}$ & $\begin{array}{r}419 \\
18\end{array}$ \\
\hline
\end{tabular}


Table K.28. Weekend P.M. peak-hour intersection volume projections for 2006-interchange at U.S. 321 (both options)-8B not opened until 8C completed

\begin{tabular}{|c|c|c|c|c|c|}
\hline \multirow[b]{2}{*}{ Intersection location } & \multirow[b]{2}{*}{ Approach } & \multirow{2}{*}{$\begin{array}{c}\text { Total } \\
\text { vehicles } \\
\text { per hour }\end{array}$} & \multicolumn{3}{|c|}{ Directional information } \\
\hline & & & $\begin{array}{c}\text { Turning } \\
\text { movement }\end{array}$ & Percent & Counts \\
\hline \multirow[t]{6}{*}{ U.S. 321 Intersection w/ Foothills Pkwy } & FH Pkwy 8A WB & 229 & Right Tum & $16.59 \%$ & 38 \\
\hline & & & Through & $7.42 \%$ & 17 \\
\hline & & & Left Tum & $75.98 \%$ & 174 \\
\hline & FH Pkwy 8B EB & 152 & Right Turn & $5.92 \%$ & 9 \\
\hline & & & Through & $51.32 \%$ & 78 \\
\hline & & & Left Turn & $42.76 \%$ & 65 \\
\hline \multirow{3}{*}{. } & U.S. $321 \mathrm{SB}$ & 650 & Right Turn & $13.69 \%$ & 89 \\
\hline & & & Through & $83.23 \%$ & 541 \\
\hline & & & Left Turn & $3.08 \%$ & 20 \\
\hline & U.S. $321 \mathrm{NB}$ & 766 & Right Turn & $14.23 \%$ & 109 \\
\hline & & & Through & $85.12 \%$ & 652 \\
\hline & & & Left Tum & $0.65 \%$ & 5 \\
\hline \multirow[t]{6}{*}{ U.S. 321 Convergence w/ SR 32} & U.S. $321 \mathrm{~EB}$ & 663 & Right Turn & $13.12 \%$ & 87 \\
\hline & & & Left Turn & $86.88 \%$ & 576 \\
\hline & U.S. $321 \mathrm{SB}$ & 431 & Right Turn & $71.23 \%$ & 307 \\
\hline & & & Through & $28.77 \%$ & 124 \\
\hline & SR $32 \mathrm{NB}$ & 69 & Through & $68.12 \%$ & 47 \\
\hline & & & Left Turn & $31.88 \%$ & 22 \\
\hline \multirow[t]{6}{*}{ U.S. 321 Intersection w/ SR 416} & SR 416 SB & 158 & Right Turn & $77.22 \%$ & 122 \\
\hline & & & Left Turn & $22.78 \%$ & 36 \\
\hline & U.S. 321 WB & 942 & Right Turn & $14.12 \%$ & 133 \\
\hline & & & Through & $85.88 \%$ & 809 \\
\hline & U.S. $321 \mathrm{~EB}$ & 453 & Through & $83.22 \%$ & 377 \\
\hline & & & Left Turn & $16.78 \%$ & 76 \\
\hline \multirow[t]{6}{*}{ SR 416 Intersection w/ Webb Creek Rd } & Webb Cr Rd WB & 43 & Right Turn & $4.65 \%$ & 2 \\
\hline & & & Left Turn & $95.35 \%$ & 41 \\
\hline & SR 416 SB & 72 & Through : & $98.61 \%$ & 71 \\
\hline & 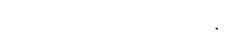 & & Left Turn & $1.39 \%$ & 1 \\
\hline & SR 416 NB & 85 & Right Turn & $45.88 \%$ & 39 \\
\hline & & & Through & $54.12 \%$ & 46 \\
\hline \multirow[t]{12}{*}{ SR 416 Intersection w/ Foothills Pkwy 8B/8C } & FH Pkwy 8B wB & 171 & Right Turn & $0.00 \%$ & 0 \\
\hline & & & Through & $100.00 \%$ & 171 \\
\hline & & & Left Tum & $0.00 \%$ & 0 \\
\hline & FH Pkwy 8C EB & 168 & Right Turn & $0.00 \%$ & 0 \\
\hline & & & Through & $100.00 \%$ & 168 \\
\hline & & & Left Turn & $0.00 \%$ & 0 \\
\hline & SR 416 SB & 112 & Right Turn & $0.00 \%$ & 0 \\
\hline & & & Through & $100.00 \%$ & 112 \\
\hline & & & Left Turn & $0.00 \%$ & 0 \\
\hline & SR 416 NB & 85 & Right Turn & $0.00 \%$ & 0 \\
\hline & & & Through & $100.00 \%$ & 85 \\
\hline & & & Left Turn & $0.00 \%$ & 0 \\
\hline
\end{tabular}


Table K.28. Weekend P.M. peak-hour intersection volume projections for 2006-interchange at U.S. 321 (directly connected)-8B not opened until 8C completed (continued)

\begin{tabular}{|c|c|c|c|c|c|}
\hline \multirow{2}{*}{ Intersection location } & \multirow[b]{2}{*}{ Approach } & \multirow{2}{*}{$\begin{array}{l}\text { Total } \\
\text { vehicles } \\
\text { per hour }\end{array}$} & \multicolumn{3}{|c|}{ Directional information } \\
\hline & & & $\begin{array}{c}\text { Tuming } \\
\text { movement }\end{array}$ & Percent & Counts \\
\hline \multirow[t]{3}{*}{ U.S. 321 Intersection w/ Foothills Pkwy 8B } & FH Pkwy 8B & 19 & $\begin{array}{l}\text { Right Tum } \\
\text { Left Tum }\end{array}$ & $\begin{array}{r}100.00 \% \\
0.00 \%\end{array}$ & $\begin{array}{r}19 \\
0\end{array}$ \\
\hline & U.S. 321 WB & 938 & $\begin{array}{l}\text { Right Turn } \\
\text { Through }\end{array}$ & $\begin{array}{r}9.06 \% \\
90.94 \%\end{array}$ & $\begin{array}{r}85 \\
853\end{array}$ \\
\hline & U.S. $321 \mathrm{~EB}$ & 410 & $\begin{array}{l}\text { Through } \\
\text { Left Turn }\end{array}$ & $\begin{array}{r}99.27 \% \\
0.73 \%\end{array}$ & $\begin{array}{r}407 \\
3\end{array}$ \\
\hline
\end{tabular}

Table K.28. Weekend P.M. peak-hour intersection volume projections for 2006-interchange at U.S. 321 (Webb Creek Rd. connection)-8B not opened until 8C completed (continued)

\begin{tabular}{|c|c|c|c|c|c|}
\hline \multirow[b]{2}{*}{ Intersection location } & \multirow[b]{2}{*}{ Approach } & \multirow{2}{*}{$\begin{array}{c}\text { Total } \\
\text { vehicles } \\
\text { per hour }\end{array}$} & \multicolumn{3}{|c|}{ Directional information } \\
\hline & & & $\begin{array}{c}\text { Turning } \\
\text { movement }\end{array}$ & Percent & Counts \\
\hline \multirow[t]{3}{*}{ Foothills Pkwy 8B/Webb Creek Rd to US 321} & FH Pkwy 8B & 69 & $\begin{array}{l}\text { Right Turn } \\
\text { Left Turn }\end{array}$ & $\begin{array}{l}63.77 \% \\
36.23 \%\end{array}$ & $\begin{array}{l}44 \\
25\end{array}$ \\
\hline & U.S. $321 \mathrm{WB}$ & 963 & $\begin{array}{l}\text { Right Turn } \\
\text { Through }\end{array}$ & $\begin{array}{l}11.42 \% \\
88.58 \%\end{array}$ & $\begin{array}{l}110 \\
853\end{array}$ \\
\hline & U.S. $321 \mathrm{~EB}$ & 435 & $\begin{array}{l}\text { Through } \\
\text { Left Turn }\end{array}$ & $\begin{array}{r}93.56 \% \\
6.44 \%\end{array}$ & $\begin{array}{r}407 \\
28\end{array}$ \\
\hline
\end{tabular}


Table K.29. Weekday A.M. peak-hour intersection volume projections for 2026 -interchange at U.S. 321 (both options)-8B not opened until 8C completed

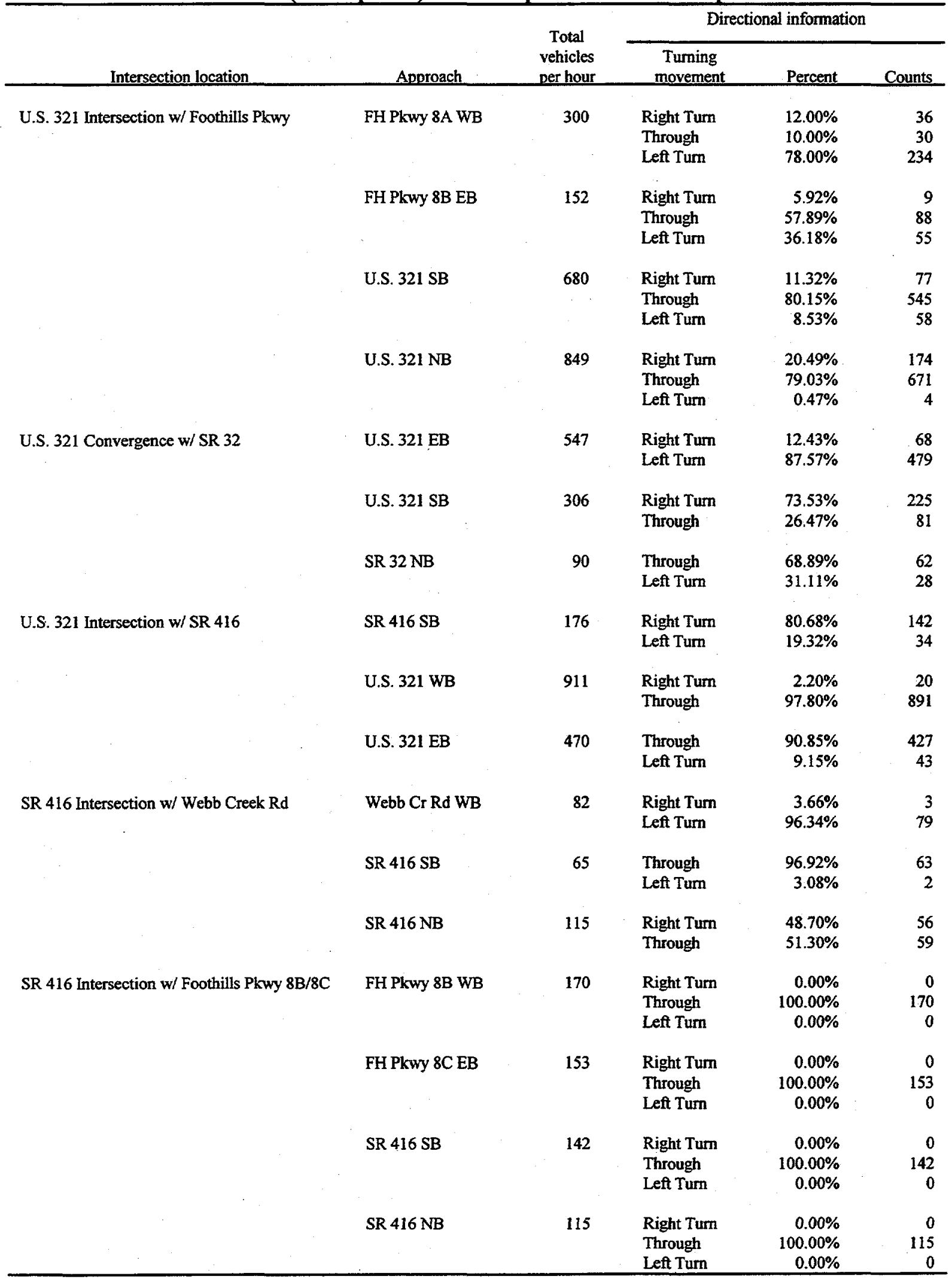


Table K.29. Weekday A.M. peak-hour intersection volume projections for 2026 -interchange at U.S. 321 (directly connected) $-8 B$ not opened until 8C completed (continued)

\begin{tabular}{|c|c|c|c|c|c|}
\hline \multirow[b]{2}{*}{ Intersection location } & \multirow[b]{2}{*}{ Approach } & \multirow{2}{*}{$\begin{array}{c}\text { Total } \\
\text { vehicles } \\
\text { per hour }\end{array}$} & \multicolumn{3}{|c|}{ Directional information } \\
\hline & & & $\begin{array}{r}\text { Turning } \\
\text { movement }\end{array}$ & Percent & Counts \\
\hline \multirow[t]{3}{*}{ U.S. 321 Intersection w/ Foothills Pkwy 8B } & FH Pkwy 8B & 2 & $\begin{array}{l}\text { Right Turn } \\
\text { Left Turn }\end{array}$ & $\begin{array}{r}100.00 \% \\
0.00 \%\end{array}$ & $\begin{array}{l}2 \\
0\end{array}$ \\
\hline & U.S. 321 WB & 968 & $\begin{array}{l}\text { Right Turn } \\
\text { Through }\end{array}$ & $\begin{array}{r}6.10 \% \\
93.91 \%\end{array}$ & $\begin{array}{r}59 \\
909\end{array}$ \\
\hline & U.S. $321 \mathrm{~EB}$ & 455 & $\begin{array}{l}\text { Through } \\
\text { Left Turn }\end{array}$ & $\begin{array}{r}99.78 \% \\
0.22 \% \\
\end{array}$ & $\begin{array}{r}454 \\
1\end{array}$ \\
\hline
\end{tabular}

Table K.29. Weekday A.M. peak-hour intersection volume projections for 2026-interchange at U.S. 321 (Webb Creek Rd. connection)-8B not opened until 8C completed (continued)

\begin{tabular}{|c|c|c|c|c|c|}
\hline \multirow[b]{2}{*}{ Intersection location } & \multirow[b]{2}{*}{ Approach } & \multirow{2}{*}{$\begin{array}{l}\text { Total } \\
\text { vehicles } \\
\text { per hour }\end{array}$} & \multicolumn{3}{|c|}{ Directional information } \\
\hline & & & $\begin{array}{c}\text { Turning } \\
\text { movement }\end{array}$ & Percent & Counts \\
\hline \multirow[t]{3}{*}{ Foothills Pkwy 8B/Webb Creek Rd to US 321} & FH Pkwy 8B & 72 & $\begin{array}{l}\text { Right Turn } \\
\text { Left Turn }\end{array}$ & $\begin{array}{l}51.39 \% \\
48.61 \%\end{array}$ & $\begin{array}{l}37 \\
35\end{array}$ \\
\hline & U.S. $321 \mathrm{WB}$ & 1003 & $\begin{array}{l}\text { Right Tum } \\
\text { Through }\end{array}$ & $\begin{array}{r}9.37 \% \\
90.63 \%\end{array}$ & $\begin{array}{r}94 \\
909\end{array}$ \\
\hline & U.S. $321 \mathrm{~EB}$ & 490 & $\begin{array}{l}\text { Through } \\
\text { Left Turn }\end{array}$ & $\begin{array}{r}92.65 \% \\
7.35 \%\end{array}$ & $\begin{array}{r}454 \\
36\end{array}$ \\
\hline
\end{tabular}


Table K.30. Weekday P.M. peak-hour intersection volume projections for 2026-interchange at U.S. 321 (both options)-8B not opened until 8C completed

\begin{tabular}{|c|c|c|c|c|c|}
\hline \multirow[b]{2}{*}{ Intersection location } & \multirow[b]{2}{*}{ Approach } & \multirow{2}{*}{$\begin{array}{c}\text { Total } \\
\text { vehicles } \\
\text { per hour }\end{array}$} & \multicolumn{3}{|c|}{ Directional information } \\
\hline & & & $\begin{array}{c}\text { Turning } \\
\text { movement }\end{array}$ & Percent & Counts \\
\hline \multirow[t]{4}{*}{ U.S. 321 Intersection w/ Foothills Pkwy } & FH Pkwy 8A WB & 221 & $\begin{array}{l}\text { Right Tum } \\
\text { Through } \\
\text { Left Turn }\end{array}$ & $\begin{array}{r}14.93 \% \\
9.50 \% \\
75.57 \%\end{array}$ & $\begin{array}{r}33 \\
21 \\
167\end{array}$ \\
\hline & FH Pkwy 8B EB & 201 & $\begin{array}{l}\text { Right Turn } \\
\text { Through } \\
\text { Left Turn }\end{array}$ & $\begin{array}{r}5.97 \% \\
57.71 \% \\
36.32 \%\end{array}$ & $\begin{array}{r}12 \\
116 \\
73\end{array}$ \\
\hline & U.S. $321 \mathrm{SB}$ & 711 & $\begin{array}{l}\text { Right Turn } \\
\text { Through } \\
\text { Left Turn }\end{array}$ & $\begin{array}{r}12.10 \% \\
85.23 \% \\
2.67 \%\end{array}$ & $\begin{array}{r}86 \\
606 \\
19\end{array}$ \\
\hline & U.S. $321 \mathrm{NB}$ & 867 & $\begin{array}{l}\text { Right Turn } \\
\text { Through } \\
\text { Left Turn }\end{array}$ & $\begin{array}{r}13.49 \% \\
85.93 \% \\
0.58 \%\end{array}$ & $\begin{array}{r}117 \\
745 \\
5\end{array}$ \\
\hline \multirow[t]{3}{*}{ U.S. 321 Convergence w/ SR 32} & U.S. $321 \mathrm{~EB}$ & 724 & $\begin{array}{l}\text { Right Turn } \\
\text { Left Turn }\end{array}$ & $\begin{array}{l}12.71 \% \\
87.29 \%\end{array}$ & $\begin{array}{r}92 \\
632\end{array}$ \\
\hline & U.S. $321 \mathrm{SB}$ & 306 & $\begin{array}{l}\text { Right Turn } \\
\text { Through }\end{array}$ & $\begin{array}{l}74.18 \% \\
25.82 \%\end{array}$ & $\begin{array}{r}227 \\
79\end{array}$ \\
\hline & SR $32 \mathrm{NB}$ & 63 & $\begin{array}{l}\text { Through } \\
\text { Left Turn }\end{array}$ & $\begin{array}{l}66.67 \% \\
33.33 \%\end{array}$ & $\begin{array}{l}42 \\
21\end{array}$ \\
\hline \multirow[t]{3}{*}{ U.S. 321 Intersection w/ SR 416} & SR $416 \mathrm{SB}$ & 116 & $\begin{array}{l}\text { Right Turn } \\
\text { Left Tum }\end{array}$ & $\begin{array}{l}83.62 \% \\
16.38 \%\end{array}$ & $\begin{array}{l}97 \\
19\end{array}$ \\
\hline & U.S. 321 WB & 885 & $\begin{array}{l}\text { Right Turn } \\
\text { Through }\end{array}$ & $\begin{array}{r}4.86 \% \\
95.14 \%\end{array}$ & $\begin{array}{r}43 \\
842\end{array}$ \\
\hline & U.S. $321 \mathrm{~EB}$ & 393 & $\begin{array}{l}\text { Through } \\
\text { Left Turn }\end{array}$ & $\begin{array}{r}94.15 \% \\
5.85 \%\end{array}$ & $\begin{array}{r}370 \\
23\end{array}$ \\
\hline \multirow[t]{3}{*}{ SR 416 Intersection w/ Webb Creek Rd } & Webb Cr Rd WB & 89 & $\begin{array}{l}\text { Right Turn } \\
\text { Left Turn }\end{array}$ & $\begin{array}{r}6.74 \% \\
93.26 \%\end{array}$ & $\begin{array}{r}6 \\
83\end{array}$ \\
\hline & SR $416 \mathrm{SB}$ & 80 & $\begin{array}{l}\text { Through } \\
\text { Left Tum }\end{array}$ & $\begin{array}{r}98.75 \% \\
1.25 \%\end{array}$ & $\begin{array}{r}79 \\
1\end{array}$ \\
\hline & SR 416 NB & 148 & $\begin{array}{l}\text { Right Turn } \\
\text { Through }\end{array}$ & $\begin{array}{l}44.59 \% \\
55.41 \%\end{array}$ & $\begin{array}{l}66 \\
82\end{array}$ \\
\hline \multirow[t]{4}{*}{ SR 416 Intersection w/ Foothills Pkwy 8B/8C } & FH Pkwy 8B WB & 172 & $\begin{array}{l}\text { Right Tum } \\
\text { Through } \\
\text { Left Turn }\end{array}$ & $\begin{array}{r}0.00 \% \\
100.00 \% \\
0.00 \%\end{array}$ & $\begin{array}{r}0 \\
172 \\
0\end{array}$ \\
\hline & FH Pkwy 8C EB & 201 & $\begin{array}{l}\text { Right Turn } \\
\text { Through } \\
\text { Left Turn }\end{array}$ & $\begin{array}{r}0.00 \% \\
100.00 \% \\
0.00 \%\end{array}$ & $\begin{array}{r}0 \\
201 \\
0\end{array}$ \\
\hline & SR 416 SB & 163 & $\begin{array}{l}\text { Right Turn } \\
\text { Through } \\
\text { Left Tum }\end{array}$ & $\begin{array}{r}0.00 \% \\
100.00 \% \\
0.00 \%\end{array}$ & $\begin{array}{r}0 \\
163 \\
0\end{array}$ \\
\hline & SR $416 \mathrm{NB}$ & 149 & $\begin{array}{l}\text { Right Turn } \\
\text { Through } \\
\text { Left Turn }\end{array}$ & $\begin{array}{r}0.00 \% \\
100.00 \% \\
0.00 \% \\
\end{array}$ & $\begin{array}{r}0 \\
149 \\
0 \\
\end{array}$ \\
\hline
\end{tabular}


Table K.30. Weekday P.M. peak-hour intersection volume projections for 2026-interchange at U.S. 321 (directly connected)-8B not opened until 8C completed (continued)

\begin{tabular}{|c|c|c|c|c|c|}
\hline \multirow[b]{2}{*}{ Intersection location } & \multirow[b]{2}{*}{ Approach } & \multirow{2}{*}{$\begin{array}{c}\text { Total } \\
\text { vehicles } \\
\text { per hour }\end{array}$} & \multicolumn{3}{|c|}{ Directional information } \\
\hline & & & $\begin{array}{c}\text { Tuming } \\
\text { movement }\end{array}$ & Percent & Counts \\
\hline \multirow[t]{3}{*}{ U.S. 321 Intersection w/ Foothills Pkwy 8B } & FH Pkwy 8B & 3 & $\begin{array}{l}\text { Right Turn } \\
\text { Left Turn }\end{array}$ & $\begin{array}{r}100.00 \% \\
0.00 \%\end{array}$ & $\begin{array}{l}3 \\
0\end{array}$ \\
\hline & U.S. 321 WB & 937 & $\begin{array}{l}\text { Right Turn } \\
\text { Through }\end{array}$ & $\begin{array}{r}8.32 \% \\
91.68 \%\end{array}$ & $\begin{array}{r}78 \\
859\end{array}$ \\
\hline & U.S. 321 EB & 395 & $\begin{array}{l}\text { Through } \\
\text { Left Turn }\end{array}$ & $\begin{array}{r}99.49 \% \\
0.51 \% \\
\end{array}$ & $\begin{array}{r}393 \\
2 \\
\end{array}$ \\
\hline
\end{tabular}

Table K.30. Weekday P.M. peak-hour intersection volume projections for 2026-interchange at U.S. 321 (Webb Creek Rd. connection) $-8 B$ not opened until 8C completed (continued)

\begin{tabular}{|c|c|c|c|c|c|}
\hline \multirow[b]{2}{*}{ Intersection location } & \multirow[b]{2}{*}{ Approach } & \multirow{2}{*}{$\begin{array}{c}\text { Total } \\
\text { vehicles } \\
\text { per hour }\end{array}$} & \multicolumn{3}{|c|}{ Directional information } \\
\hline & & & $\begin{array}{l}\text { Turning } \\
\text { movement }\end{array}$ & Percent & Counts \\
\hline \multirow[t]{3}{*}{ Foothills Pkwy 8B/Webb Creek Rd to US 321} & FH Pkwy 8B & 83 & $\begin{array}{l}\text { Right Turn } \\
\text { Left Turn }\end{array}$ & $\begin{array}{l}51.81 \% \\
48.19 \%\end{array}$ & $\begin{array}{l}43 \\
40\end{array}$ \\
\hline & U.S. 321 WB & 977 & $\begin{array}{l}\text { Right Tum } \\
\text { Through }\end{array}$ & $\begin{array}{l}12.08 \% \\
87.92 \%\end{array}$ & $\begin{array}{l}118 \\
859\end{array}$ \\
\hline & U.S. $321 \mathrm{~EB}$ & 435 & $\begin{array}{l}\text { Through } \\
\text { Left Turn }\end{array}$ & $\begin{array}{r}90.34 \% \\
9.66 \%\end{array}$ & $\begin{array}{r}393 \\
42\end{array}$ \\
\hline
\end{tabular}


Table K.31. Weekend A.M. peak-hour intersection volume projections for 2026-interchange at U.S. 321 (both options) $-8 B$ not opened until 8C completed

\begin{tabular}{|c|c|c|c|c|c|}
\hline \multirow[b]{2}{*}{ Intersection location } & \multirow[b]{2}{*}{ Approach } & \multirow{2}{*}{$\begin{array}{c}\text { Total } \\
\text { vehicles } \\
\text { per hour }\end{array}$} & \multicolumn{3}{|c|}{ Directional information } \\
\hline & & & $\begin{array}{c}\text { Turning } \\
\text { movement }\end{array}$ & Percent & Counts \\
\hline \multirow[t]{4}{*}{ U.S. 321 Intersection w/ Foothills Pkwy } & FH Pkwy 8A WB & 507 & $\begin{array}{l}\text { Right Turn } \\
\text { Through } \\
\text { Left Tum }\end{array}$ & $\begin{array}{r}12.03 \% \\
9.86 \% \\
78.11 \%\end{array}$ & $\begin{array}{r}61 \\
50 \\
396\end{array}$ \\
\hline & FH Pkwy 8B EB & 208 & $\begin{array}{l}\text { Right Turn } \\
\text { Through } \\
\text { Left Turn }\end{array}$ & $\begin{array}{r}5.77 \% \\
57.69 \% \\
36.54 \%\end{array}$ & $\begin{array}{r}12 \\
120 \\
76\end{array}$ \\
\hline & U.S. $321 \mathrm{SB}$ & 749 & $\begin{array}{l}\text { Right Turn } \\
\text { Through } \\
\text { Left Turn }\end{array}$ & $\begin{array}{r}11.35 \% \\
79.97 \% \\
8.68 \%\end{array}$ & $\begin{array}{r}85 \\
599 \\
65\end{array}$ \\
\hline & U.S. $321 \mathrm{NB}$ & 1046 & $\begin{array}{l}\text { Right Tum } \\
\text { Through } \\
\text { Left Tum }\end{array}$ & $\begin{array}{r}20.65 \% \\
78.87 \% \\
0.48 \%\end{array}$ & $\begin{array}{r}216 \\
825 \\
5\end{array}$ \\
\hline \multirow[t]{3}{*}{ U.S. 321 Convergence w/ SR 32} & U.S. $321 \mathrm{~EB}$ & 749 & $\begin{array}{l}\text { Right Turn } \\
\text { Left Turn }\end{array}$ & $\begin{array}{l}12.42 \% \\
87.58 \%\end{array}$ & $\begin{array}{r}93 \\
656\end{array}$ \\
\hline & U.S. $321 \mathrm{SB}$ & 696 & $\begin{array}{l}\text { Right Turn } \\
\text { Through }\end{array}$ & $\begin{array}{l}73.56 \% \\
26.44 \%\end{array}$ & $\begin{array}{l}512 \\
184\end{array}$ \\
\hline & SR $32 \mathrm{NB}$ & 123 & $\begin{array}{l}\text { Through } \\
\text { Left Turn }\end{array}$ & $\begin{array}{l}69.92 \% \\
30.08 \%\end{array}$ & $\begin{array}{l}86 \\
37\end{array}$ \\
\hline \multirow[t]{3}{*}{ U.S. 321 Intersection w/ SR 416} & SR $416 \mathrm{SB}$ & 191 & $\begin{array}{l}\text { Right Turn } \\
\text { Left Turn }\end{array}$ & $\begin{array}{l}81.15 \% \\
18.85 \%\end{array}$ & $\begin{array}{r}155 \\
36\end{array}$ \\
\hline & U.S. $321 \mathrm{WB}$ & 1106 & $\begin{array}{l}\text { Right Turn } \\
\text { Through }\end{array}$ & $\begin{array}{r}2.26 \% \\
97.74 \%\end{array}$ & $\begin{array}{r}25 \\
1081\end{array}$ \\
\hline & U.S. $321 \mathrm{~EB}$ & 597 & $\begin{array}{l}\text { Through } \\
\text { Left Turn }\end{array}$ & $\begin{array}{r}90.79 \% \\
9.21 \%\end{array}$ & $\begin{array}{r}542 \\
55\end{array}$ \\
\hline \multirow[t]{3}{*}{ SR 416 Intersection w/ Webb Creek Rd } & Webb Cr Rd WB & 52 & $\begin{array}{l}\text { Right Turn } \\
\text { Left Tum }\end{array}$ & $\begin{array}{r}3.85 \% \\
96.15 \%\end{array}$ & $\begin{array}{r}2 \\
50\end{array}$ \\
\hline & SR 416 SB & 48 & $\begin{array}{l}\text { Through } \\
\text { Left Turn }\end{array}$ & $\begin{array}{r}95.83 \% \\
4.17 \%\end{array}$ & $\begin{array}{r}46 \\
2\end{array}$ \\
\hline & SR $416 \mathrm{NB}$ & 96 & $\begin{array}{l}\text { Right Turn } \\
\text { Through }\end{array}$ & $\begin{array}{l}47.92 \% \\
52.08 \%\end{array}$ & $\begin{array}{l}46 \\
50\end{array}$ \\
\hline \multirow[t]{4}{*}{ SR 416 Intersection w/ Foothills Pkwy 8B/8C } & FH Pkwy 8B WB & 221 & $\begin{array}{l}\text { Right Turn } \\
\text { Through } \\
\text { Left Turn }\end{array}$ & $\begin{array}{r}0.00 \% \\
100.00 \% \\
0.00 \%\end{array}$ & 221 \\
\hline & FH Pkwy 8C EB & 209 & $\begin{array}{l}\text { Right Turn } \\
\text { Through } \\
\text { Left Tum }\end{array}$ & $\begin{array}{r}0.00 \% \\
100.00 \% \\
0.00 \%\end{array}$ & 209 \\
\hline & SR $416 \mathrm{SB}$ & 96 & $\begin{array}{l}\text { Right Turn } \\
\text { Through } \\
\text { Left Tum }\end{array}$ & $\begin{array}{r}0.00 \% \\
100.00 \% \\
0.00 \%\end{array}$ & 96 \\
\hline & SR $416 \mathrm{NB}$ & 96 & $\begin{array}{l}\text { Right Turn } \\
\text { Through } \\
\text { Left Turn }\end{array}$ & $\begin{array}{r}0.00 \% \\
100.00 \% \\
0.00 \% \\
\end{array}$ & 96 \\
\hline
\end{tabular}


Table K.31. Weekend A.M. peak-hour intersection volume projections for 2026-interchange at U.S. 321 (directly connected) -8B not opened until 8C completed (continued)

\begin{tabular}{|c|c|c|c|c|c|}
\hline \multirow[b]{2}{*}{ Intersection location } & \multirow[b]{2}{*}{ Approach } & \multirow{2}{*}{$\begin{array}{c}\text { Total } \\
\text { vehicles } \\
\text { per hour }\end{array}$} & \multicolumn{3}{|c|}{ Directional information } \\
\hline & & & $\begin{array}{c}\text { Turning } \\
\text { movement }\end{array}$ & Percent & Counts \\
\hline \multirow[t]{3}{*}{ U.S. 321 Intersection w/ Foothills Pkwy 8B } & FH Pkwy 8B & 3 & $\begin{array}{l}\text { Right Turn } \\
\text { Left Tum }\end{array}$ & $\begin{array}{r}100.00 \% \\
0.00 \%\end{array}$ & $\begin{array}{l}3 \\
0\end{array}$ \\
\hline & U.S. $321 \mathrm{WB}$ & 1184 & $\begin{array}{l}\text { Right Turn } \\
\text { Through }\end{array}$ & $\begin{array}{r}6.84 \% \\
93.16 \%\end{array}$ & $\begin{array}{r}81 \\
1103\end{array}$ \\
\hline & U.S. $321 \mathrm{~EB}$ & 578 & $\begin{array}{l}\text { Through } \\
\text { Left Tum }\end{array}$ & $\begin{array}{r}99.65 \% \\
0.35 \%\end{array}$ & $\begin{array}{r}576 \\
2\end{array}$ \\
\hline
\end{tabular}

Table K.31. Weekend A.M. peak-hour intersection volume projections for 2026-interchange at U.S. 321 (Webb Creek Rd. connection)-8B not opened until 8C completed (continued)

\begin{tabular}{|c|c|c|c|c|c|}
\hline \multirow[b]{2}{*}{ Intersection location } & \multirow[b]{2}{*}{ Approach } & \multirow{2}{*}{$\begin{array}{c}\text { Total } \\
\text { vehicles } \\
\text { per hour }\end{array}$} & \multicolumn{3}{|c|}{ Directional information } \\
\hline & & & $\begin{array}{c}\text { Turning } \\
\text { movement }\end{array}$ & Percent & Counts \\
\hline \multirow[t]{3}{*}{ Foothills Pkwy 8B/Webb Creek Rd to US 321} & FH Pkwy 8B & 53 & $\begin{array}{l}\text { Right Tum } \\
\text { Left Turn }\end{array}$ & $\begin{array}{l}52.83 \% \\
47.17 \%\end{array}$ & $\begin{array}{l}28 \\
25\end{array}$ \\
\hline & U.S. 321 WB & 1209 & $\begin{array}{l}\text { Right Turn } \\
\text { Through }\end{array}$ & $\begin{array}{r}8.77 \% \\
91.23 \%\end{array}$ & $\begin{array}{r}106 \\
1103\end{array}$ \\
\hline & U.S. $321 \mathrm{~EB}$ & 603 & $\begin{array}{l}\text { Through } \\
\text { Left Turn }\end{array}$ & $\begin{array}{r}95.52 \% \\
4.48 \%\end{array}$ & $\begin{array}{r}576 \\
27\end{array}$ \\
\hline
\end{tabular}


Table K.32. Weekend P.M. peak-hour intersection volume projections for 2026-interchange at U.S. 321 (both options)-8B not opened until 8C completed

\begin{tabular}{|c|c|c|c|c|c|}
\hline \multirow[b]{2}{*}{ Intersection location } & \multirow[b]{2}{*}{ Approach } & \multirow{2}{*}{$\begin{array}{c}\text { Total } \\
\text { vehicles } \\
\text { per hour }\end{array}$} & \multicolumn{3}{|c|}{ Directional information } \\
\hline & & & $\begin{array}{c}\text { Turning } \\
\text { movement }\end{array}$ & Percent & Counts \\
\hline \multirow[t]{12}{*}{ U.S. 321 Intersection w/ Foothills Pkwy } & FH Pkwy 8A WB & 308 & Right Turn & $15.26 \%$ & 47 \\
\hline & & & Through & $9.42 \%$ & 29 \\
\hline & & & Left Turn & $75.32 \%$ & 232 \\
\hline & FH Pkwy 8B EB & 241 & Right Turn & $5.81 \%$ & 14 \\
\hline & & & Through & $57.68 \%$ & 139 \\
\hline & & & Left Tum & $36.51 \%$ & 88 \\
\hline & U.S. $321 \mathrm{SB}$ & 833 & Right Turn & $12.12 \%$ & 101 \\
\hline & & & Through & $85.23 \%$ & 710 \\
\hline & & & Left Turn & $2.64 \%$ & 22 \\
\hline & U.S. $321 \mathrm{NB}$ & 991 & Right Tum & $13.42 \%$ & 133 \\
\hline & & & Through & $86.07 \%$ & 853 \\
\hline & & & Left Turn & $0.50 \%$ & 5 \\
\hline \multirow[t]{6}{*}{ U.S. 321 Convergence w/ SR 32} & U.S. $321 \mathrm{~EB}$ & 871 & Right Turn & $12.74 \%$ & 111 \\
\hline & & & Left Turn & $87.26 \%$ & 760 \\
\hline & U.S. 321 SB & 580 & Right Tum & $73.97 \%$ & 429 \\
\hline & & & Through & $26.03 \%$ & 151 \\
\hline & SR $32 \mathrm{NB}$ & 82 & Through & $65.85 \%$ & 54 \\
\hline & & & Left Tum & $34.15 \%$ & 28 \\
\hline \multirow[t]{6}{*}{ U.S. 321 Intersection w/SR 416} & SR $416 \mathrm{SB}$ & 223 & Right Turn & $82.96 \%$ & 185 \\
\hline & & & Left Turn & $17.04 \%$ & 38 \\
\hline & U.S. 321 WB & 1183 & Right Turn & $4.82 \%$ & 57 \\
\hline & & & Through & $95.18 \%$ & 1126 \\
\hline & U.S. $321 \mathrm{~EB}$ & 560 & Through & $94.11 \%$ & 527 \\
\hline & & & Left Turn & $5.89 \%$ & 33 \\
\hline \multirow[t]{6}{*}{ SR 416 Intersection w/ Webb Creek Rd } & Webb Cr Rd WB & 68 & Right Turn & $7.35 \%$ & 5 \\
\hline & & & Left Turn & $92.65 \%$ & 63 \\
\hline & SR 416 SB & 110 & Through & $99.09 \%$ & 109 \\
\hline & & & Left Turn & $0.91 \%$ & 1 \\
\hline & SR $416 \mathrm{NB}$ & 148 & Right Tum & $44.59 \%$ & 66 \\
\hline & & & Through & $55.41 \%$ & 82 \\
\hline \multirow[t]{12}{*}{ SR 416 Intersection w/ Foothills Pkwy 8B/8C } & FH Pkwy 8B WB & 207 & Right Turn & $0.00 \%$ & 0 \\
\hline & & & Through & $100.00 \%$ & 207 \\
\hline & & & Left Turn & $0.00 \%$ & 0 \\
\hline & FH Pkwy 8C EB & 242 & Right Tum & $0.00 \%$ & 0 \\
\hline & & & Through & $100.00 \%$ & 242 \\
\hline & & & Left Turn & $0.00 \%$ & 0 \\
\hline & SR 416 SB & 171 & Right Tum & $0.00 \%$ & 0 \\
\hline & & & Through & $100.00 \%$ & 171 \\
\hline & & & Left Turn & $0.00 \%$ & 0 \\
\hline & SR $416 \mathrm{NB}$ & 149 & Right Turn & $0.00 \%$ & 0 \\
\hline & & & Through & $100.00 \%$ & 149 \\
\hline & & & Left Turn & $0.00 \%$ & 0 \\
\hline
\end{tabular}


Table K.32. Weekend P.M. peak-hour intersection volume projections for 2026 -interchange at U.S. 321 (directly connected) $-8 \mathrm{~B}$ not opened until 8C completed (continued)

\begin{tabular}{|c|c|c|c|c|c|}
\hline \multirow[b]{2}{*}{ Intersection location } & \multirow[b]{2}{*}{ Approach } & \multirow{2}{*}{$\begin{array}{l}\text { Total } \\
\text { vehicles } \\
\text { per hour }\end{array}$} & \multicolumn{3}{|c|}{ Directional information } \\
\hline & & & $\begin{array}{c}\text { Turning } \\
\text { movement }\end{array}$ & Percent & Counts \\
\hline \multirow[t]{3}{*}{ U.S. 321 Intersection w/ Foothills Pkwy $8 \mathrm{~B}$} & FH Pkwy 8B & 4 & $\begin{array}{l}\text { Right Turn } \\
\text { Left Tum }\end{array}$ & $\begin{array}{r}100.00 \% \\
0.00 \%\end{array}$ & $\begin{array}{l}4 \\
0\end{array}$ \\
\hline & U.S. 321 WB & 1243 & $\begin{array}{l}\text { Right Turn } \\
\text { Through }\end{array}$ & $\begin{array}{r}7.56 \% \\
92.44 \%\end{array}$ & $\begin{array}{r}94 \\
1149\end{array}$ \\
\hline & U.S. $321 \mathrm{~EB}$ & 562 & $\begin{array}{l}\text { Through } \\
\text { Left Turn }\end{array}$ & $\begin{array}{r}99.64 \% \\
0.36 \%\end{array}$ & $\begin{array}{r}560 \\
2\end{array}$ \\
\hline
\end{tabular}

Table K.32. Weekend P.M. peak-hour intersection volume projections for 2026 -interchange at U.S. 321 (Webb Creek Rd. connection)-8B not opened until 8C completed (continued)

\begin{tabular}{|c|c|c|c|c|c|}
\hline \multirow[b]{2}{*}{ Intersection location } & \multirow[b]{2}{*}{ Approach } & \multirow{2}{*}{$\begin{array}{c}\text { Total } \\
\text { vehicles } \\
\text { per hour }\end{array}$} & \multicolumn{3}{|c|}{ Directional information } \\
\hline & & & $\begin{array}{c}\text { Turning } \\
\text { movement }\end{array}$ & Percent & Counts \\
\hline \multirow[t]{3}{*}{ Foothills Pkwy 8B/Webb Creek Rd to US 321} & FH Pkwy 8B & 74 & $\begin{array}{l}\text { Right Turn } \\
\text { Left Turn }\end{array}$ & $\begin{array}{l}52.70 \% \\
47.30 \%\end{array}$ & $\begin{array}{l}39 \\
35\end{array}$ \\
\hline & U.S. 321 WB & 1278 & $\begin{array}{l}\text { Right Turn } \\
\text { Through }\end{array}$ & $\begin{array}{l}10.09 \% \\
89.91 \%\end{array}$ & $\begin{array}{r}129 \\
1149\end{array}$ \\
\hline & U.S. $321 \mathrm{~EB}$ & 597 & $\begin{array}{l}\text { Through } \\
\text { Left Turn }\end{array}$ & $\begin{array}{r}93.80 \% \\
6.20 \%\end{array}$ & $\begin{array}{r}560 \\
37\end{array}$ \\
\hline
\end{tabular}


Table K.33. Weekday A.M. peak-hour intersection volume projections for 2006 -interchange at Highway 416 (north ramp option)-8B opened prior to completion of $8 \mathrm{C}$

\begin{tabular}{|c|c|c|c|c|c|}
\hline \multirow[b]{2}{*}{ Intersection location } & \multirow[b]{2}{*}{ Approach } & \multirow{2}{*}{$\begin{array}{c}\text { Total } \\
\text { vehicles } \\
\text { per hour }\end{array}$} & \multicolumn{3}{|c|}{ Directional information } \\
\hline & & & $\begin{array}{c}\text { Turning } \\
\text { movement }\end{array}$ & Percent & Counts \\
\hline \multirow[t]{4}{*}{ U.S. 321 Intersection w/ Foothills Pkwy } & FH Pkwy 8A WB & 222 & $\begin{array}{l}\text { Right Tum } \\
\text { Through } \\
\text { Left Tum }\end{array}$ & $\begin{array}{r}13.06 \% \\
7.66 \% \\
79.28 \%\end{array}$ & $\begin{array}{r}29 \\
17 \\
176\end{array}$ \\
\hline & FH Pkwy 8B EB & 96 & $\begin{array}{l}\text { Right Turn } \\
\text { Through } \\
\text { Left Tum }\end{array}$ & $\begin{array}{r}6.25 \% \\
51.04 \% \\
42.71 \%\end{array}$ & $\begin{array}{r}6 \\
49 \\
41\end{array}$ \\
\hline & U.S. $321 \mathrm{SB}$ & 536 & $\begin{array}{l}\text { Right Tum } \\
\text { Through } \\
\text { Left Tum }\end{array}$ & $\begin{array}{r}12.69 \% \\
77.43 \% \\
9.89 \%\end{array}$ & $\begin{array}{r}68 \\
415 \\
53\end{array}$ \\
\hline & U.S. $321 \mathrm{NB}$ & 660 & $\begin{array}{l}\text { Right Tum } \\
\text { Through } \\
\text { Left Turn }\end{array}$ & $\begin{array}{r}21.67 \% \\
77.73 \% \\
0.61 \%\end{array}$ & $\begin{array}{r}143 \\
513 \\
4\end{array}$ \\
\hline \multirow[t]{3}{*}{ U.S. 321 Convergence w/ SR 32} & U.S. 321 EB & 416 & $\begin{array}{l}\text { Right Turn } \\
\text { Left Turn }\end{array}$ & $\begin{array}{l}12.74 \% \\
87.26 \%\end{array}$ & $\begin{array}{r}53 \\
363\end{array}$ \\
\hline & U.S. 321 SB & 227 & $\begin{array}{l}\text { Right Turn } \\
\text { Through }\end{array}$ & $\begin{array}{l}70.93 \% \\
29.07 \%\end{array}$ & $\begin{array}{r}161 \\
66\end{array}$ \\
\hline & SR 32 NB & 76 & $\begin{array}{l}\text { Through } \\
\text { Left Turn }\end{array}$ & $\begin{array}{l}71.05 \% \\
28.95 \%\end{array}$ & $\begin{array}{l}54 \\
22\end{array}$ \\
\hline \multirow[t]{3}{*}{ U.S. 321 Intersection w/ SR 416} & $\mathrm{SR} 416 \mathrm{SB}$ & 226 & $\begin{array}{l}\text { Right Turn } \\
\text { Left Tum }\end{array}$ & $\begin{array}{l}86.73 \% \\
13.27 \%\end{array}$ & $\begin{array}{r}196 \\
30\end{array}$ \\
\hline & U.S. 321 WB & 734 & $\begin{array}{l}\text { Right Turn } \\
\text { Through }\end{array}$ & $\begin{array}{r}4.63 \% \\
95.37 \%\end{array}$ & $\begin{array}{r}34 \\
700\end{array}$ \\
\hline & U.S. $321 \mathrm{~EB}$ & 518 & $\begin{array}{l}\text { Through } \\
\text { Left Tum }\end{array}$ & $\begin{array}{l}58.88 \% \\
41.12 \%\end{array}$ & $\begin{array}{l}305 \\
213\end{array}$ \\
\hline \multirow[t]{4}{*}{ SR 416 Intersection w/ Webb Creek Rd } & Webb Cr Rd WB & 54 & $\begin{array}{l}\text { Right Turn } \\
\text { Through } \\
\text { Left Turn }\end{array}$ & $\begin{array}{r}3.70 \% \\
0.00 \% \\
96.30 \%\end{array}$ & $\begin{array}{r}2 \\
0 \\
52\end{array}$ \\
\hline & SR $416 \mathrm{SB}$ & 42 & $\begin{array}{l}\text { Right Tum } \\
\text { Through } \\
\text { Left Tum }\end{array}$ & $\begin{array}{r}0.00 \% \\
97.62 \% \\
2.38 \%\end{array}$ & $\begin{array}{r}0 \\
41 \\
1\end{array}$ \\
\hline & SR $416 \mathrm{NB}$ & 162 & $\begin{array}{l}\text { Right Tum } \\
\text { Through } \\
\text { Left Tum }\end{array}$ & $\begin{array}{l}20.37 \% \\
20.37 \% \\
59.26 \%\end{array}$ & $\begin{array}{l}33 \\
33 \\
96\end{array}$ \\
\hline & FH Pkwy ramp & 84 & $\begin{array}{l}\text { Right Tum } \\
\text { Through } \\
\text { Left Turn }\end{array}$ & $\begin{array}{r}100.00 \% \\
0.00 \% \\
0.00 \%\end{array}$ & $\begin{array}{r}84 \\
0 \\
0\end{array}$ \\
\hline \multirow[t]{4}{*}{ SR 416 Intersection w/ Foothills Pkwy 8B/8C } & FH Pkwy 8B WB & 84 & Through & $100.00 \%$ & 84 \\
\hline & FH Pkwy 8C EB & 0 & Through & & 0 \\
\hline & SR $416 \mathrm{SB}$ & 93 & Through & $100.00 \%$ & 93 \\
\hline & SR $416 \mathrm{NB}$ & 162 & Through & $100.00 \%$ & 162 \\
\hline
\end{tabular}


Table K.34. Weekday P.M. peak-hour intersection volume projections for 2006 -interchange at Highway 416 (north ramp option)-8B opened prior to completion of $8 \mathrm{C}$

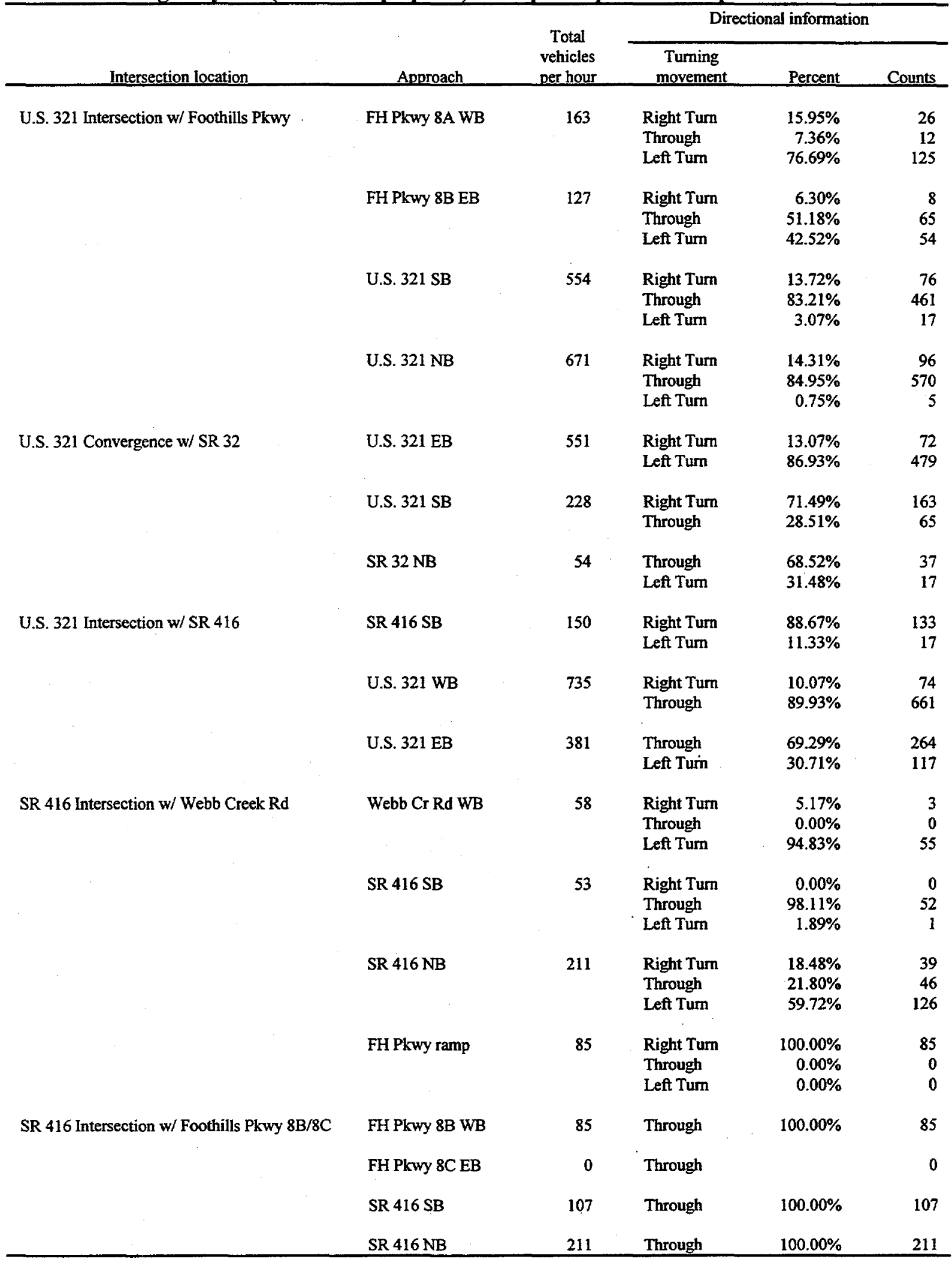


Table K.35. Weekend A.M. peak-hour intersection volume projections for 2006 -interchange at Highway 416 (north ramp option)-8B opened prior to completion of $8 \mathrm{C}$

\begin{tabular}{|c|c|c|c|c|c|}
\hline \multirow[b]{2}{*}{ Intersection location } & \multirow[b]{2}{*}{ Approach } & \multirow{2}{*}{$\begin{array}{c}\text { Total } \\
\text { vehicles } \\
\text { per hour }\end{array}$} & \multicolumn{3}{|c|}{ Directional information } \\
\hline & & & $\begin{array}{c}\text { Turning } \\
\text { movement }\end{array}$ & Percent & Counts \\
\hline \multirow[t]{4}{*}{ U.S. 321 Intersection w/ Foothills Pkwy } & FH Pkwy 8A WB & 375 & $\begin{array}{l}\text { Right Turn } \\
\text { Through } \\
\text { Left Tumn }\end{array}$ & $\begin{array}{r}13.07 \% \\
7.73 \% \\
79.20 \%\end{array}$ & $\begin{array}{r}49 \\
29 \\
297\end{array}$ \\
\hline & FH Pkwy 8B EB & 131 & $\begin{array}{l}\text { Right Turn } \\
\text { Through } \\
\text { Left Turn }\end{array}$ & $\begin{array}{r}6.11 \% \\
51.15 \% \\
42.75 \%\end{array}$ & $\begin{array}{r}8 \\
67 \\
56\end{array}$ \\
\hline & U.S. $321 \mathrm{SB}$ & 590 & $\begin{array}{l}\text { Right Turn } \\
\text { Through } \\
\text { Left Turn }\end{array}$ & $\begin{array}{l}12.71 \% \\
77.29 \% \\
10.00 \%\end{array}$ & $\begin{array}{r}75 \\
456 \\
59\end{array}$ \\
\hline & U.S. $321 \mathrm{NB}$ & 813 & $\begin{array}{l}\text { Right Turn } \\
\text { Through } \\
\text { Left Turn }\end{array}$ & $\begin{array}{r}21.77 \% \\
77.61 \% \\
0.62 \%\end{array}$ & $\begin{array}{r}177 \\
631 \\
5\end{array}$ \\
\hline \multirow[t]{3}{*}{ U.S. 321 Convergence w/ SR 32} & U.S. $321 \mathrm{~EB}$ & 570 & $\begin{array}{l}\text { Right Turn } \\
\text { Left Turn }\end{array}$ & $\begin{array}{l}12.81 \% \\
87.19 \%\end{array}$ & $\begin{array}{r}73 \\
497\end{array}$ \\
\hline & U.S. $321 \mathrm{SB}$ & 518 & $\begin{array}{l}\text { Right Turn } \\
\text { Through }\end{array}$ & $\begin{array}{l}70.85 \% \\
29.15 \%\end{array}$ & $\begin{array}{l}367 \\
151\end{array}$ \\
\hline & SR $32 \mathrm{NB}$ & 104 & $\begin{array}{l}\text { Through } \\
\text { Left Turn }\end{array}$ & $\begin{array}{l}72.12 \% \\
27.88 \%\end{array}$ & $\begin{array}{l}75 \\
29\end{array}$ \\
\hline \multirow[t]{3}{*}{ U.S. 321 Intersection w/ SR 416} & SR 416 SB & 245 & $\begin{array}{l}\text { Right Tum } \\
\text { Left Tum }\end{array}$ & $\begin{array}{l}86.94 \% \\
13.06 \%\end{array}$ & $\begin{array}{r}213 \\
32\end{array}$ \\
\hline & U.S. 321 WB & 892 & $\begin{array}{l}\text { Right Turn } \\
\text { Through }\end{array}$ & $\begin{array}{r}4.82 \% \\
95.18 \%\end{array}$ & $\begin{array}{r}43 \\
849\end{array}$ \\
\hline & U.S. $321 \mathrm{~EB}$ & 661 & $\begin{array}{l}\text { Through } \\
\text { Left Turn }\end{array}$ & $\begin{array}{l}58.55 \% \\
41.45 \%\end{array}$ & $\begin{array}{l}387 \\
274\end{array}$ \\
\hline \multirow[t]{4}{*}{ SR 416 Intersection w/ Webb Creek Rd } & Webb Cr Rd WB & 34 & $\begin{array}{l}\text { Right Tum } \\
\text { Through } \\
\text { Left Tum }\end{array}$ & $\begin{array}{r}2.94 \% \\
0.00 \% \\
97.06 \%\end{array}$ & $\begin{array}{r}1 \\
0 \\
33\end{array}$ \\
\hline & SR 416 SB & 31 & $\begin{array}{l}\text { Right Turn } \\
\text { Through } \\
\text { Left Tum }\end{array}$ & $\begin{array}{r}0.00 \% \\
96.77 \% \\
3.23 \%\end{array}$ & $\begin{array}{r}0 \\
30 \\
1\end{array}$ \\
\hline & SR $416 \mathrm{NB}$ & 186 & $\begin{array}{l}\text { Right Tum } \\
\text { Through } \\
\text { Left Turn }\end{array}$ & $\begin{array}{l}14.52 \% \\
15.05 \% \\
70.43 \%\end{array}$ & $\begin{array}{r}27 \\
28 \\
131\end{array}$ \\
\hline & FH Pkwy ramp & 109 & $\begin{array}{l}\text { Right Turn } \\
\text { Through } \\
\text { Left Turn }\end{array}$ & $\begin{array}{r}100.00 \% \\
0.00 \% \\
0.00 \%\end{array}$ & $\begin{array}{r}109 \\
0 \\
0\end{array}$ \\
\hline \multirow[t]{4}{*}{ SR 416 Intersection w/ Foothills Pkwy $8 \mathrm{~B} / 8 \mathrm{C}$} & FH Pkwy 8B WB & 109 & Through & $100.00 \%$ & 109 \\
\hline & FH Pkwy 8C EB & 0 & Through & & 0 \\
\hline & SR $416 \mathrm{SB}$ & 63 & Through & $100.00 \%$ & 63 \\
\hline & SR $416 \mathrm{NB}$ & 186 & Through & $100.00 \%$ & 186 \\
\hline
\end{tabular}


Table K.36. Weekend P.M. peak-hour intersection volume projections for 2006 -interchange at Highway 416 (north ramp option) $-8 B$ opened prior to completion of $8 \mathrm{C}$

\begin{tabular}{|c|c|c|c|c|c|}
\hline \multirow[b]{2}{*}{ Intersection location } & \multirow[b]{2}{*}{ Approach } & \multirow{2}{*}{$\begin{array}{c}\text { Total } \\
\text { vehicles } \\
\text { per hour }\end{array}$} & \multicolumn{3}{|c|}{ Directional information } \\
\hline & & & $\begin{array}{c}\text { Turning } \\
\text { movement }\end{array}$ & Percent & Counts \\
\hline \multirow[t]{4}{*}{ U.S. 321 Intersection w/ Foothills Pkwy } & FH Pkwy 8A WB & 229 & $\begin{array}{l}\text { Right Tum } \\
\text { Through } \\
\text { Left Tum }\end{array}$ & $\begin{array}{r}16.59 \% \\
7.42 \% \\
75.98 \%\end{array}$ & $\begin{array}{r}38 \\
17 \\
174\end{array}$ \\
\hline & FH Pkwy 8B EB & 152 & $\begin{array}{l}\text { Right Turn } \\
\text { Through } \\
\text { Left Turn }\end{array}$ & $\begin{array}{r}5.92 \% \\
51.32 \% \\
42.76 \%\end{array}$ & $\begin{array}{r}9 \\
78 \\
65\end{array}$ \\
\hline & U.S. $321 \mathrm{SB}$ & 650 & $\begin{array}{l}\text { Right Turn } \\
\text { Through } \\
\text { Left Tum }\end{array}$ & $\begin{array}{r}13.69 \% \\
83.23 \% \\
3.08 \%\end{array}$ & $\begin{array}{r}89 \\
541 \\
20\end{array}$ \\
\hline & U.S. $321 \mathrm{NB}$ & 766 & $\begin{array}{l}\text { Right Turn } \\
\text { Through } \\
\text { Left Turn }\end{array}$ & $\begin{array}{r}14.23 \% \\
85.12 \% \\
0.65 \%\end{array}$ & $\begin{array}{r}109 \\
652 \\
5\end{array}$ \\
\hline \multirow[t]{3}{*}{ U.S. 321 Convergence w/ SR 32} & U.S. $321 \mathrm{~EB}$ & 663 & $\begin{array}{l}\text { Right Turn } \\
\text { Left Turn }\end{array}$ & $\begin{array}{l}13.12 \% \\
86.88 \%\end{array}$ & $\begin{array}{r}87 \\
576\end{array}$ \\
\hline & U.S. $321 \mathrm{SB}$ & 431 & $\begin{array}{l}\text { Right Turn } \\
\text { Through }\end{array}$ & $\begin{array}{l}71.23 \% \\
28.77 \%\end{array}$ & $\begin{array}{l}307 \\
124\end{array}$ \\
\hline & SR $32 \mathrm{NB}$ & 69 & $\begin{array}{l}\text { Through } \\
\text { Left Turn }\end{array}$ & $\begin{array}{l}68.12 \% \\
31.88 \%\end{array}$ & $\begin{array}{l}47 \\
22\end{array}$ \\
\hline \multirow[t]{3}{*}{ U.S. 321 Intersection w/ SR 416} & SR 416 SB & 289 & $\begin{array}{l}\text { Right Turn } \\
\text { Left Turn }\end{array}$ & $\begin{array}{l}88.24 \% \\
11.76 \%\end{array}$ & $\begin{array}{r}255 \\
34\end{array}$ \\
\hline & U.S. 321 WB & 982 & $\begin{array}{l}\text { Right Turn } \\
\text { Through }\end{array}$ & $\begin{array}{r}9.98 \% \\
90.02 \%\end{array}$ & $\begin{array}{r}98 \\
884\end{array}$ \\
\hline & U.S. $321 \mathrm{~EB}$ & 538 & $\begin{array}{l}\text { Through } \\
\text { Left Turn }\end{array}$ & $\begin{array}{l}69.89 \% \\
30.11 \%\end{array}$ & $\begin{array}{l}376 \\
162\end{array}$ \\
\hline \multirow[t]{4}{*}{ SR 416 Intersection w/ Webb Creek Rd } & Webb Cr Rd WB & 43 & $\begin{array}{l}\text { Right Turn } \\
\text { Through } \\
\text { Left Tum }\end{array}$ & $\begin{array}{r}4.65 \% \\
0.00 \% \\
95.35 \%\end{array}$ & $\begin{array}{r}2 \\
0 \\
41\end{array}$ \\
\hline & SR 416 SB & 72 & $\begin{array}{l}\text { Right Turn } \\
\text { Through } \\
\text { Left Turn }\end{array}$ & $\begin{array}{r}0.00 \% \\
98.61 \% \\
1.39 \%\end{array}$ & $\begin{array}{r}0 \\
71 \\
1\end{array}$ \\
\hline & SR $416 \mathrm{NB}$ & 237 & $\begin{array}{l}\text { Right Turn } \\
\text { Through } \\
\text { Left Turn }\end{array}$ & $\begin{array}{l}16.46 \% \\
19.41 \% \\
64.14 \%\end{array}$ & $\begin{array}{r}39 \\
46 \\
152\end{array}$ \\
\hline & FH Pkwy ramp & 102 & $\begin{array}{l}\text { Right Tum } \\
\text { Through } \\
\text { Left Turn }\end{array}$ & $\begin{array}{r}100.00 \% \\
0.00 \% \\
0.00 \%\end{array}$ & $\begin{array}{r}102 \\
0 \\
0\end{array}$ \\
\hline \multirow[t]{4}{*}{ SR 416 Intersection w/ Foothills Pkwy 8B/8C } & FH Pkwy 8B WB & 102 & Through & $100.00 \%$ & 102 \\
\hline & FH Pkwy 8C EB & 0 & Through & & 0 \\
\hline & SR 416 SB & 112 & Through & $100.00 \%$ & 112 \\
\hline & SR $416 \mathrm{NB}$ & 237 & Through & $100.00 \%$ & 237 \\
\hline
\end{tabular}


Table K.37. Weekday A.M. peak-hour intersection volume projections for 2026-interchange at Highway 416 (north ramp option) $-8 \mathrm{~B}$ opened prior to completion of $8 \mathrm{C}$

\begin{tabular}{|c|c|c|c|c|c|}
\hline \multirow{2}{*}{ Intersection location } & \multirow[b]{2}{*}{ Approach } & \multirow{2}{*}{$\begin{array}{c}\text { Total } \\
\text { vehicles } \\
\text { per hour }\end{array}$} & \multicolumn{3}{|c|}{ Directional information } \\
\hline & & & $\begin{array}{c}\text { Turning } \\
\text { movement }\end{array}$ & Percent & Counts \\
\hline \multirow[t]{12}{*}{ U.S. 321 Intersection w/ Foothills Pkwy } & FH Pkwy 8A WB & 300 & Right Tum & $12.00 \%$ & 36 \\
\hline & & & Through & $10.00 \%$ & 30 \\
\hline & & & Left Turn & $78.00 \%$ & 234 \\
\hline & FH Pkwy 8B EB & 152 & Right Turn & $5.92 \%$ & 9 \\
\hline & & & Through & $57.89 \%$ & 88 \\
\hline & & & Left Turn & $36.18 \%$ & 55 \\
\hline & U.S. 321 SB & 680 & Right Turn & $11.32 \%$ & 77 \\
\hline & & & Through & $80.15 \%$ & 545 \\
\hline & & & Left Turn & $8.53 \%$ & 58 \\
\hline & U.S. $321 \mathrm{NB}$ & 849 & Right Turn & $20.49 \%$ & 174 \\
\hline & & & Through & $79.03 \%$ & 671 \\
\hline & & & Left Turn & $0.47 \%$ & 4 \\
\hline \multirow[t]{6}{*}{ U.S. 321 Convergence w/ SR 32} & U.S. $321 \mathrm{~EB}$ & 547 & Right Turn & $12.43 \%$ & 68 \\
\hline & & & Left Turn & $87.57 \%$ & 479 \\
\hline & U.S. $321 \mathrm{SB}$ & 306 & Right Turn & $73.53 \%$ & 225 \\
\hline & & & Through & $26.47 \%$ & 81 \\
\hline & SR 32 NB & 90 & Through & $68.89 \%$ & 62 \\
\hline & & & Left Tum & $31.11 \%$ & 28 \\
\hline \multirow[t]{6}{*}{ U.S. 321 Intersection w/ SR 416} & SR 416 SB & 305 & Right Turn & $89.18 \%$ & 272 \\
\hline & & & Left Turn & $10.82 \%$ & 33 \\
\hline & U.S. 321 WB & 975 & Right Tum & $1.85 \%$ & 18 \\
\hline & & & Through & $98.15 \%$ & 957 \\
\hline & U.S. $321 \mathrm{~EB}$ & 632 & Through & $67.41 \%$ & 426 \\
\hline & & & Left Turn & $32.59 \%$ & 206 \\
\hline \multirow[t]{9}{*}{ SR 416 Intersection w/ Webb Creek Rd } & Webb Cr Rd WB & 82 & Right Turn & $3.66 \%$ & 3 \\
\hline & & & Through & $0.00 \%$ & 0 \\
\hline & & & Left Turn & $96.34 \%$ & 79 \\
\hline & SR $416 \mathrm{SB}$ & 65 & Right Tum & $0.00 \%$ & 0 \\
\hline & & & Through & $96.92 \%$ & 63 \\
\hline & & & Left Turn & $3.08 \%$ & 2 \\
\hline & SR $416 \mathrm{NB}$ & 267 & Right Turn & $20.97 \%$ & 56 \\
\hline & & & Through & $22.10 \%$ & 59 \\
\hline & & & Left Turn & $56.93 \%$ & 152 \\
\hline \multirow{3}{*}{. } & FH Pkwy ramp & 108 & Right Turn & $100.00 \%$ & 108 \\
\hline & & & Through & $0.00 \%$ & 0 \\
\hline & & & Left Turn & $0.00 \%$ & 0 \\
\hline \multirow[t]{2}{*}{ SR 416 Intersection w/ Foothills Pkwy 8B/8C } & FH Pkwy 8B WB & 108 & Through & $100.00 \%$ & 108 \\
\hline & FH Pkwy 8C EB & 0 & Through & & 0 \\
\hline & SR $416 \mathrm{SB}$ & 142 & Through & $100.00 \%$ & 142 \\
\hline & SR $416 \mathrm{NB}$ & 267 & Through & $100.00 \%$ & 267 \\
\hline
\end{tabular}


Table K.38. Weekday P.M. peak-hour intersection volume projections for 2026-interchange at Highway 416 (north ramp option)-8B opened prior to completion of $8 \mathrm{C}$

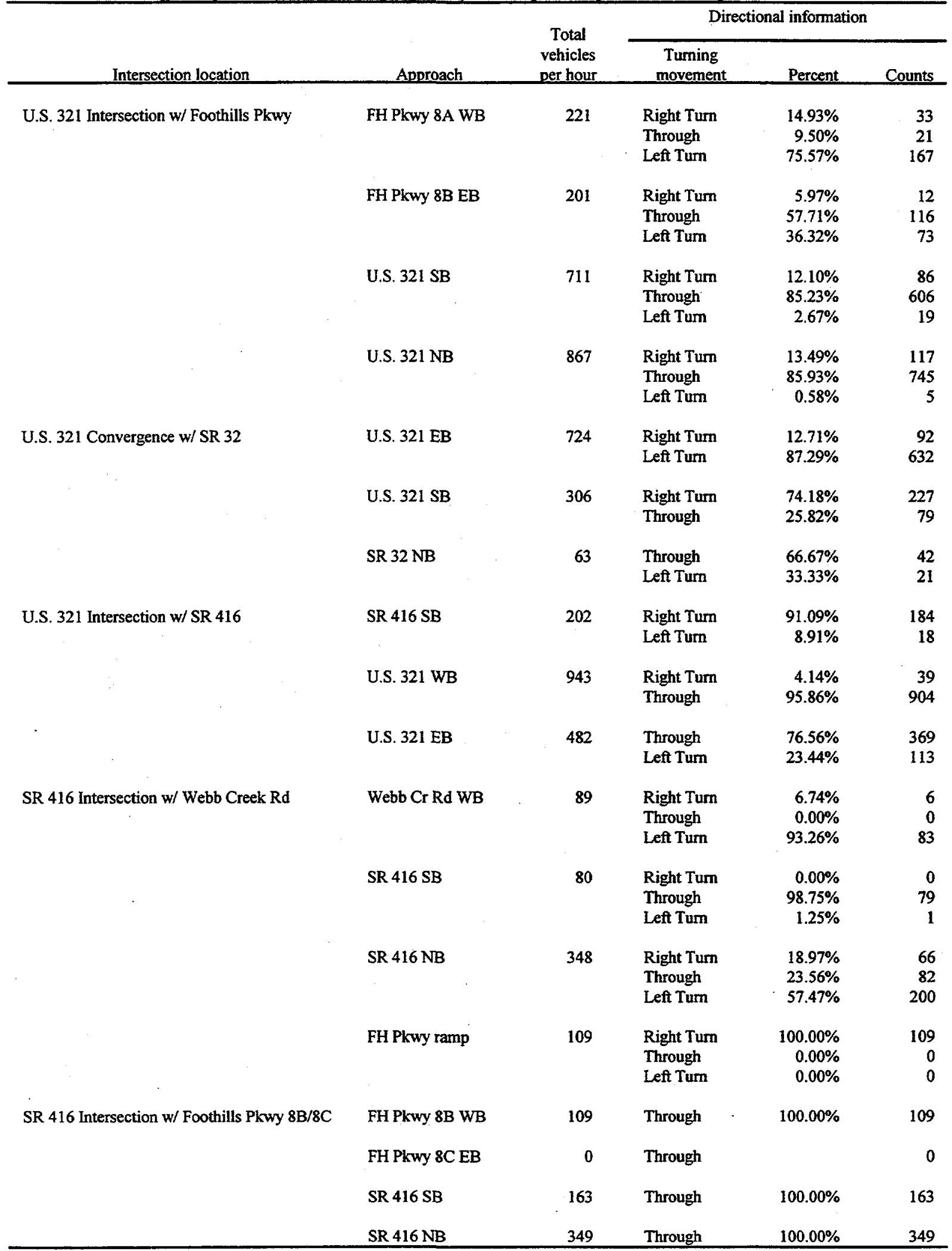


Table K.39. Weekend A.M. peak-hour intersection volume projections for 2026-interchange at Highway 416 (north ramp option)-8B opened prior to completion of $8 \mathrm{C}$

\begin{tabular}{|c|c|c|c|c|c|}
\hline \multirow[b]{2}{*}{ Intersection location } & \multirow[b]{2}{*}{ Approach } & \multirow{2}{*}{$\begin{array}{c}\text { Total } \\
\text { vehicles } \\
\text { per hour }\end{array}$} & \multicolumn{3}{|c|}{ Directional information } \\
\hline & & & $\begin{array}{c}\text { Turning } \\
\text { movement }\end{array}$ & Percent & Counts \\
\hline \multirow[t]{12}{*}{ U.S. 321 Intersection w/ Foothills Pkwy } & FH Pkwy 8A WB & 507 & Right Turn & $12.03 \%$ & 61 \\
\hline & & & Through & $9.86 \%$ & 50 \\
\hline & & & Left Turn & $78.11 \%$ & 396 \\
\hline & FH Pkwy 8B EB & 208 & Right Tum & $5.77 \%$ & 12 \\
\hline & & & Through & $57.69 \%$ & 120 \\
\hline & & & Left Tum & $36.54 \%$ & 76 \\
\hline & U.S. $321 \mathrm{SB}$ & 749 & Right Turn & $11.35 \%$ & 85 \\
\hline & & & Through & $79.97 \%$ & 599 \\
\hline & & & Left Turn & $8.68 \%$ & 65 \\
\hline & U.S. $321 \mathrm{NB}$ & 1046 & Right Turn & $20.65 \%$ & 216 \\
\hline & & & Through & $78.87 \%$ & 825 \\
\hline & & & Left Turn & $0.48 \%$ & 5 \\
\hline \multirow[t]{6}{*}{ U.S. 321 Convergence w/ SR 32} & U.S. $321 \mathrm{~EB}$ & 749 & Right Tum & $12.42 \%$ & 93 \\
\hline & & & Left Tum & $87.58 \%$ & 656 \\
\hline & U.S. $321 \mathrm{SB}$ & 696 & Right Tum & $73.56 \%$ & 512 \\
\hline & & & Through & $26.44 \%$ & 184 \\
\hline & SR $32 \mathrm{NB}$ & 123 & Through & $69.92 \%$ & 86 \\
\hline & & & Left Tum & $30.08 \%$ & 37 \\
\hline \multirow[t]{6}{*}{ U.S. 321 Intersection w/ SR 416} & SR 416 SB & 331 & Right Turn & $89.43 \%$ & 296 \\
\hline & & & Left Turn & $10.57 \%$ & 35 \\
\hline & U.S. $321 \mathrm{WB}$ & 1184 & Right Turn & $1.94 \%$ & 23 \\
\hline & & & Through & $98.06 \%$ & 1161 \\
\hline & U.S. $321 \mathrm{~EB}$ & 806 & Through & $67.12 \%$ & 541 \\
\hline & & & Left Turn & $32.88 \%$ & 265 \\
\hline \multirow[t]{12}{*}{ SR 416 Intersection w/ Webb Creek Rd } & Webb Cr Rd WB & 52 & Right Tum & $3.85 \%$ & 2 \\
\hline & & & Through & $0.00 \%$ & 0 \\
\hline & & & Left Tum & $96.15 \%$ & 50 \\
\hline & SR 416 SB & 48 & Right Tum & $0.00 \%$ & 0 \\
\hline & & & Through & $95.83 \%$ & 46 \\
\hline & & & Left Tum & $4.17 \%$ & 2 \\
\hline & SR $416 \mathrm{NB}$ & 304 & Right Turn & $15.13 \%$ & 46 \\
\hline & & & Through & $16.45 \%$ & 50 \\
\hline & & & Left Turn & $68.42 \%$ & 208 \\
\hline & FH Pkwy ramp & 140 & Right Turn & $100.00 \%$ & 140 \\
\hline & & & Through & $0.00 \%$ & 0 \\
\hline & & & Left Tum & $0.00 \%$ & 0 \\
\hline \multirow[t]{4}{*}{ SR 416 Intersection w/ Foothills Pkwy 8B/8C } & FH Pkwy 8B WB & 140 & Through & $100.00 \%$ & 140 \\
\hline & FH Pkwy 8C EB & 0 & Through & & 0 \\
\hline & SR $416 \mathrm{SB}$ & 96 & Through & $100.00 \%$ & 96 \\
\hline & SR $416 \mathrm{NB}$ & 304 & Through & $100.00 \%$ & 304 \\
\hline
\end{tabular}


Table K.40. Weekend P.M. peak-hour intersection volume projections for 2026-interchange at Highway 416 (north ramp option)-8B opened prior to completion of $8 \mathrm{C}$

\begin{tabular}{|c|c|c|c|c|c|}
\hline \multirow[b]{2}{*}{ Intersection location } & \multirow[b]{2}{*}{ Approach } & \multirow{2}{*}{$\begin{array}{c}\text { Total } \\
\text { vehicles } \\
\text { per hour }\end{array}$} & \multicolumn{3}{|c|}{ Directional information } \\
\hline & & & $\begin{array}{c}\text { Tuming } \\
\text { movement }\end{array}$ & Percent & Counts \\
\hline \multirow[t]{3}{*}{ U.S. 321 Intersection w/ Foothills Pkwy } & FH Pkwy 8A WB & 308 & Right Turn & $15.26 \%$ & 47 \\
\hline & & & Through & $9.42 \%$ & 29 \\
\hline & & & Left Tum & $75.32 \%$ & 232 \\
\hline \multirow{9}{*}{. } & FH Pkwy 8B EB & 241 & Right Turn & $5.81 \%$ & 14 \\
\hline & & & Through & $57.68 \%$ & 139 \\
\hline & & & Left Tum & $36.51 \%$ & 88 \\
\hline & U.S. 321 SB & 833 & Right Turn & $12.12 \%$ & $10 I$ \\
\hline & & & Through & $85.23 \%$ & 710 \\
\hline & & & Left Turn & $2.64 \%$ & 22 \\
\hline & U.S. $321 \mathrm{NB}$ & 991 & Right Turn & $13.42 \%$ & 133 \\
\hline & & & Through & $86.07 \%$ & 853 \\
\hline & & & Left Tum & $0.50 \%$ & 5 \\
\hline \multirow[t]{6}{*}{ U.S. 321 Convergence w/ SR 32} & U.S. 321 EB & 871 & Right Turn & $12.74 \%$ & 111 \\
\hline & & & Left Tum & $87.26 \%$ & 760 \\
\hline & U.S. 321 SB & 580 & Right Turn & $73.97 \%$ & 429 \\
\hline & & & Through & $26.03 \%$ & 151 \\
\hline & SR 32 NB & 82 & Through & $65.85 \%$ & 54 \\
\hline & & & Left Turn & $34.15 \%$ & 28 \\
\hline \multirow[t]{6}{*}{ U.S. 321 Intersection w/ SR 416} & SR 416 SB & 391 & Right Tum & $90.54 \%$ & 354 \\
\hline & & & Left Tum & $9.46 \%$ & 37 \\
\hline & U.S. 321 WB & 1262 & Right Turn & $4.20 \%$ & 53 \\
\hline & & & Through & $95.80 \%$ & 1209 \\
\hline & U.S. 321 EB & 683 & Through & $77.01 \%$ & 526 \\
\hline & & & Left Turn & $22.99 \%$ & 157 \\
\hline \multirow[t]{12}{*}{ SR 416 Intersection w/ Webb Creek Rd } & Webb Cr Rd WB & 68 & Right Turn & $7.35 \%$ & 5 \\
\hline & & & Through & $0.00 \%$ & 0 \\
\hline & & & Left Tum & $92.65 \%$ & 63 \\
\hline & SR $416 \mathrm{SB}$ & 110 & Right Turn & $0.00 \%$ & 0 \\
\hline & & & Through & $99.09 \%$ & 109 \\
\hline & & & Left Tum & $0.91 \%$ & 1 \\
\hline & SR $416 \mathrm{NB}$ & 389 & Right Turn & $16.97 \%$ & 66 \\
\hline & & & Through & $21.08 \%$ & 82 \\
\hline & & & Left Tum & $61.95 \%$ & 241 \\
\hline & FH Pkwy ramp & 131 & Right Turn & $100.00 \%$ & 131 \\
\hline & & & Through & $0.00 \%$ & 0 \\
\hline & & & Left Tum & $0.00 \%$ & 0 \\
\hline \multirow[t]{4}{*}{ SR 416 Intersection w/ Foothills Pkwy 8B/8C } & FH Pkwy 8B WB & 131 & Through & $100.00 \%$ & 131 \\
\hline & FH Pkwy 8C EB & 0 & Through & & 0 \\
\hline & SR $416 \mathrm{SB}$ & 171 & Through & $100.00 \%$ & 171 \\
\hline & SR $416 \mathrm{NB}$ & 390 & Through & $100.00 \%$ & 390 \\
\hline
\end{tabular}


Table K.41. Weekday A.M. peak-hour intersection volume projections for 2006-interchange at Highway 416 (south ramp option)-8B opened prior to completion of $8 \mathrm{C}$

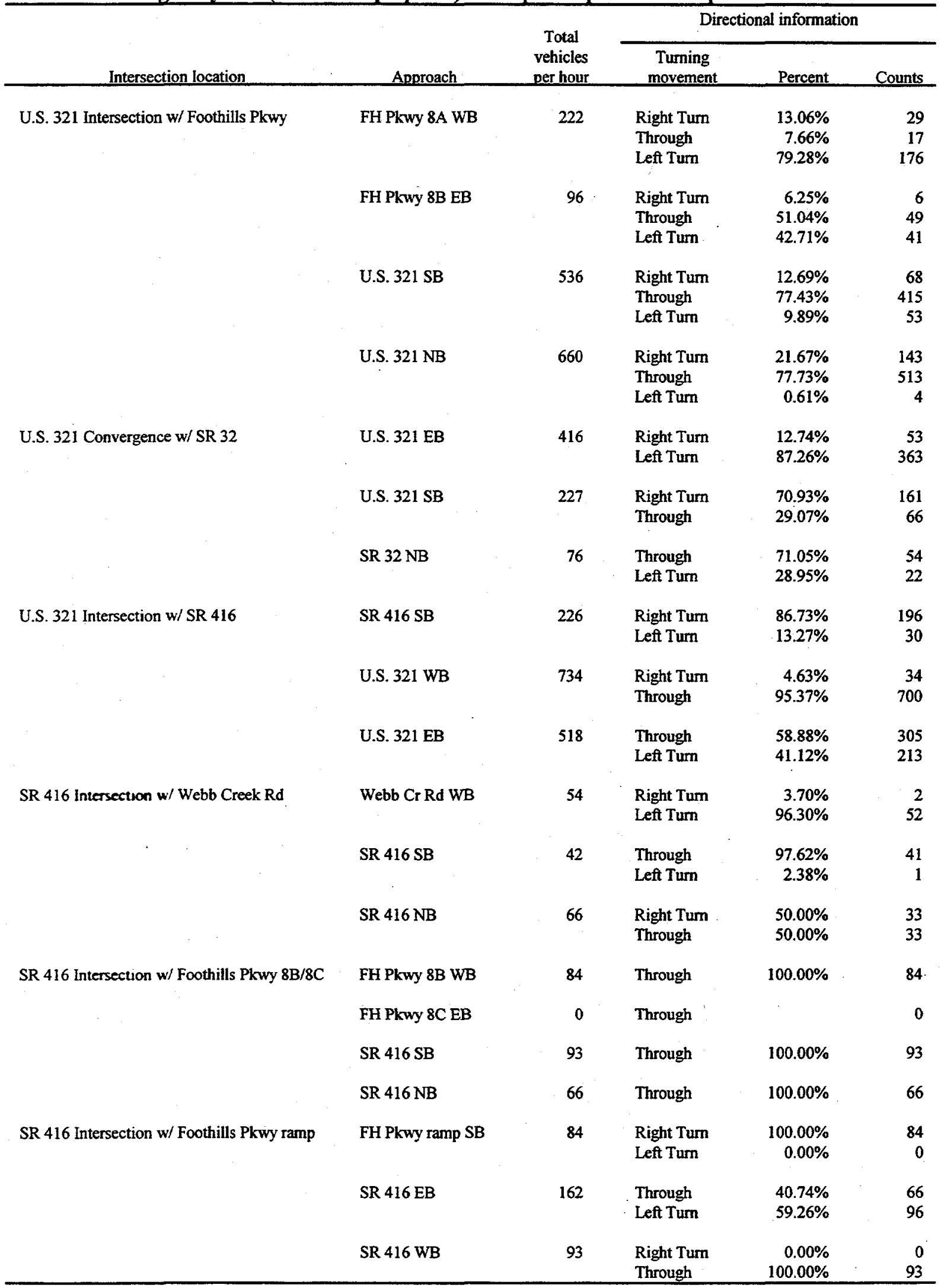


Table K.42. Weekday P.M. peak-hour intersection volume projections for 2006 -interchange at Highway 416 (south ramp option) $-8 B$ opened prior to completion of $8 \mathrm{C}$

\begin{tabular}{|c|c|c|c|c|c|}
\hline \multirow[b]{2}{*}{ Intersection location } & \multirow[b]{2}{*}{ Approach } & \multirow{2}{*}{$\begin{array}{c}\text { Total } \\
\text { vehicles } \\
\text { per hour }\end{array}$} & \multicolumn{3}{|c|}{ Directional information } \\
\hline & & & $\begin{array}{c}\text { Turning } \\
\text { movement }\end{array}$ & Percent & Counts \\
\hline \multirow[t]{4}{*}{ U.S. 321 Intersection w/ Foothills Pkwy } & FH Pkwy 8A WB & 163 & $\begin{array}{l}\text { Right Turn } \\
\text { Through } \\
\text { Left Turn }\end{array}$ & $\begin{array}{r}15.95 \% \\
7.36 \% \\
76.69 \%\end{array}$ & $\begin{array}{r}26 \\
12 \\
125\end{array}$ \\
\hline & FH Pkwy 8B EB & 127 & $\begin{array}{l}\text { Right Tum } \\
\text { Through } \\
\text { Left Turn }\end{array}$ & $\begin{array}{r}6.30 \% \\
51.18 \% \\
42.52 \%\end{array}$ & $\begin{array}{r}8 \\
65 \\
54\end{array}$ \\
\hline & U.S. $321 \mathrm{SB}$ & 554 & $\begin{array}{l}\text { Right Tum } \\
\text { Through } \\
\text { Left Turn }\end{array}$ & $\begin{array}{r}13.72 \% \\
83.21 \% \\
3.07 \%\end{array}$ & $\begin{array}{r}76 \\
461 \\
17\end{array}$ \\
\hline & U.S. $321 \mathrm{NB}$ & 671 & $\begin{array}{l}\text { Right Turn } \\
\text { Through } \\
\text { Left Tum }\end{array}$ & $\begin{array}{r}14.31 \% \\
84.95 \% \\
0.75 \%\end{array}$ & $\begin{array}{r}96 \\
570 \\
5\end{array}$ \\
\hline \multirow[t]{3}{*}{ U.S. 321 Convergence w/ SR 32} & U.S. $321 \mathrm{~EB}$ & 551 & $\begin{array}{l}\text { Right Turn } \\
\text { Left Turn }\end{array}$ & $\begin{array}{l}13.07 \% \\
86.93 \%\end{array}$ & $\begin{array}{r}72 \\
479\end{array}$ \\
\hline & U.S. $321 \mathrm{SB}$ & 228 & $\begin{array}{l}\text { Right Turn } \\
\text { Through }\end{array}$ & $\begin{array}{l}71.49 \% \\
28.51 \%\end{array}$ & $\begin{array}{r}163 \\
65\end{array}$ \\
\hline & SR $32 \mathrm{NB}$ & 54 & $\begin{array}{l}\text { Through } \\
\text { Left Turn }\end{array}$ & $\begin{array}{l}68.52 \% \\
31.48 \%\end{array}$ & $\begin{array}{l}37 \\
17\end{array}$ \\
\hline \multirow[t]{3}{*}{ U.S. 321 Intersection w/ SR 416} & SR 416 SB & 150 & $\begin{array}{l}\text { Right Turn } \\
\text { Left Turn }\end{array}$ & $\begin{array}{l}88.67 \% \\
11.33 \%\end{array}$ & $\begin{array}{r}133 \\
17\end{array}$ \\
\hline & U.S. $321 \mathrm{WB}$ & 735 & $\begin{array}{l}\text { Right Turn } \\
\text { Through }\end{array}$ & $\begin{array}{l}10.07 \% \\
89.93 \%\end{array}$ & $\begin{array}{r}74 \\
661\end{array}$ \\
\hline & U.S. $321 \mathrm{~EB}$ & 381 & $\begin{array}{l}\text { Through } \\
\text { Left Turn }\end{array}$ & $\begin{array}{l}69.29 \% \\
30.71 \%\end{array}$ & $\begin{array}{l}264 \\
117\end{array}$ \\
\hline \multirow[t]{3}{*}{ SR 416 Intersection w/ Webb Creek Rd } & Webb Cr Rd WB & 58 & $\begin{array}{l}\text { Right Turn } \\
\text { Left Turn }\end{array}$ & $\begin{array}{r}5.17 \% \\
94.83 \%\end{array}$ & $\begin{array}{r}3 \\
55\end{array}$ \\
\hline & SR $416 \mathrm{SB}$ & 53 & $\begin{array}{l}\text { Through } \\
\text { Left Tum }\end{array}$ & $\begin{array}{r}98.11 \% \\
1.89 \%\end{array}$ & $\begin{array}{r}52 \\
1\end{array}$ \\
\hline & SR $416 \mathrm{NB}$ & 85 & $\begin{array}{l}\text { Right Turn } \\
\text { Through }\end{array}$ & $\begin{array}{l}45.88 \% \\
54.12 \%\end{array}$ & $\begin{array}{l}39 \\
46\end{array}$ \\
\hline \multirow[t]{4}{*}{ SR 416 Intersection w/ Foothills Pkwy 8B/8C } & FH Pkwy 8B WB & 85 & Through & $100.00 \%$ & 85 \\
\hline & FH Pkwy 8C EB & 0 & Through & & 0 \\
\hline & SR 416 SB & 107 & Through & $100.00 \%$ & 107 \\
\hline & SR $416 \mathrm{NB}$ & 85 & Through & $100.00 \%$ & 85 \\
\hline \multirow[t]{3}{*}{ SR 416 Intersection w/ Foothills Pkwy ramp } & FH Pkwy ramp SB & 85 & $\begin{array}{l}\text { Right Turn } \\
\text { Left Turn }\end{array}$ & $\begin{array}{r}100.00 \% \\
0.00 \%\end{array}$ & $\begin{array}{r}85 \\
0\end{array}$ \\
\hline & SR 416 EB & 211 & $\begin{array}{l}\text { Through } \\
\text { Left Turn }\end{array}$ & $\begin{array}{l}40.28 \% \\
59.72 \%\end{array}$ & $\begin{array}{r}85 \\
126\end{array}$ \\
\hline & SR 416 WB & 107 & $\begin{array}{l}\text { Right Turn } \\
\text { Through }\end{array}$ & $\begin{array}{r}0.00 \% \\
100.00 \%\end{array}$ & $\begin{array}{r}0 \\
107\end{array}$ \\
\hline
\end{tabular}


Table K.43. Weekend A.M. peak-hour intersection volume projections for 2006-interchange at Highway 416 (south ramp option)-8B opened prior to completion of $8 \mathrm{C}$

\begin{tabular}{|c|c|c|c|c|c|}
\hline \multirow{2}{*}{ Intersection location } & \multirow[b]{2}{*}{ Approach } & \multirow{2}{*}{$\begin{array}{l}\text { Total } \\
\text { vehicles } \\
\text { per hour }\end{array}$} & \multicolumn{3}{|c|}{ Directional information } \\
\hline & & & $\begin{array}{c}\text { Turning } \\
\text { movement }\end{array}$ & Percent & Counts \\
\hline \multirow[t]{4}{*}{ U.S. 321 Intersection w/ Foothills Pkwy } & FH Pkwy 8A WB & 375 & $\begin{array}{l}\text { Right Turn } \\
\text { Through } \\
\text { Left Turn }\end{array}$ & $\begin{array}{r}13.07 \% \\
7.73 \% \\
79.20 \%\end{array}$ & $\begin{array}{r}49 \\
29 \\
297\end{array}$ \\
\hline & FH Pkwy 8B EB & 131 & $\begin{array}{l}\text { Right Turn } \\
\text { Through } \\
\text { Left Turn }\end{array}$ & $\begin{array}{r}6.11 \% \\
51.15 \% \\
42.75 \%\end{array}$ & $\begin{array}{r}8 \\
67 \\
56\end{array}$ \\
\hline & U.S. $321 \mathrm{SB}$ & 590 & $\begin{array}{l}\text { Right Tum } \\
\text { Through } \\
\text { Left Tum }\end{array}$ & $\begin{array}{l}12.71 \% \\
77.29 \% \\
10.00 \%\end{array}$ & $\begin{array}{r}75 \\
456 \\
59\end{array}$ \\
\hline & U.S. $321 \mathrm{NB}$ & 813 & $\begin{array}{l}\text { Right Tum } \\
\text { Through } \\
\text { Left Turn }\end{array}$ & $\begin{array}{r}21.77 \% \\
77.61 \% \\
0.62 \%\end{array}$ & $\begin{array}{r}177 \\
631 \\
5\end{array}$ \\
\hline \multirow[t]{3}{*}{ U.S. 321 Convergence w/ SR 32} & U.S. $321 \mathrm{~EB}$ & 570 & $\begin{array}{l}\text { Right Tum } \\
\text { Left Turn }\end{array}$ & $\begin{array}{l}12.81 \% \\
87.19 \%\end{array}$ & $\begin{array}{r}73 \\
497\end{array}$ \\
\hline & U.S. $321 \mathrm{SB}$ & 518 & $\begin{array}{l}\text { Right Tum } \\
\text { Through }\end{array}$ & $\begin{array}{l}70.85 \% \\
29.15 \%\end{array}$ & $\begin{array}{l}367 \\
151\end{array}$ \\
\hline & SR 32 NB & 104 & $\begin{array}{l}\text { Through } \\
\text { Left Turn }\end{array}$ & $\begin{array}{l}72.12 \% \\
27: 88 \%\end{array}$ & $\begin{array}{l}75 \\
29\end{array}$ \\
\hline \multirow[t]{3}{*}{ U.S. 321 Intersection w/ SR 416} & SR 416 SB & 245 & $\begin{array}{l}\text { Right Turn } \\
\text { Left Turn }\end{array}$ & $\begin{array}{l}86.94 \% \\
13.06 \%\end{array}$ & $\begin{array}{r}213 \\
32\end{array}$ \\
\hline & U.S. $321 \mathrm{WB}$ & 892 & $\begin{array}{l}\text { Right Turn } \\
\text { Through }\end{array}$ & $\begin{array}{r}4.82 \% \\
95.18 \%\end{array}$ & $\begin{array}{r}43 \\
849\end{array}$ \\
\hline & U.S. $321 \mathrm{~EB}$ & 661 & $\begin{array}{l}\text { Through } \\
\text { Left Turn }\end{array}$ & $\begin{array}{l}58.55 \% \\
41.45 \%\end{array}$ & $\begin{array}{l}387 \\
274\end{array}$ \\
\hline \multirow[t]{3}{*}{ SR 416 Intersection w/ Webb Creek Rd } & Webb Cr Rd WB & 34 & $\begin{array}{l}\text { Right Turn } \\
\text { Left Turn }\end{array}$ & $\begin{array}{r}2.94 \% \\
97.06 \%\end{array}$ & $\begin{array}{r}1 \\
33\end{array}$ \\
\hline & SR 416 SB & 31 & $\begin{array}{l}\text { Through } \\
\text { Left Turn }\end{array}$ & $\begin{array}{r}96.77 \% \\
3.23 \%\end{array}$ & $\begin{array}{r}30 \\
1\end{array}$ \\
\hline & SR 416 NB & 55 & $\begin{array}{l}\text { Right Turn } \\
\text { Through }\end{array}$ & $\begin{array}{l}49.09 \% \\
50.91 \%\end{array}$ & $\begin{array}{l}27 \\
28\end{array}$ \\
\hline \multirow[t]{4}{*}{ SR 416 Intersection w/ Foothills Pkwy 8B/8C } & FH Pkwy 8B WB & 109 & Through & $100.00 \%$ & 109 \\
\hline & FH Pkwy 8C EB & 0 & Through & & 0 \\
\hline & SR 416 SB & 63 & Through & $100.00 \%$ & 63 \\
\hline & SR $416 \mathrm{NB}$ & 55 & Through & $100.00 \%$ & 55 \\
\hline \multirow[t]{3}{*}{ SR 416 Intersection w/ Foothills Pkwy ramp } & FH Pkwy ramp SB & 109 & $\begin{array}{l}\text { Right Turn } \\
\text { Left Tum }\end{array}$ & $\begin{array}{r}100.00 \% \\
0.00 \%\end{array}$ & $\begin{array}{r}109 \\
0\end{array}$ \\
\hline & SR $416 \mathrm{~EB}$ & 186 & $\begin{array}{l}\text { Through } \\
\text { Left Turn }\end{array}$ & $\begin{array}{l}29.57 \% \\
70.43 \%\end{array}$ & $\begin{array}{r}55 \\
131\end{array}$ \\
\hline & SR 416 WB & 63 & $\begin{array}{l}\text { Right Turn } \\
\text { Through }\end{array}$ & $\begin{array}{r}0.00 \% \\
100.00 \%\end{array}$ & $\begin{array}{r}0 \\
63\end{array}$ \\
\hline
\end{tabular}


Table K.44. Weekend P.M. peak-hour intersection volume projections for 2006 -interchange at Highway 416 (south ramp option)-8B opened prior to completion of $8 \mathrm{C}$

\begin{tabular}{|c|c|c|c|c|c|}
\hline \multirow[b]{2}{*}{ Intersection location } & \multirow[b]{2}{*}{ Approach } & \multirow{2}{*}{$\begin{array}{c}\text { Total } \\
\text { vehicles } \\
\text { per hour }\end{array}$} & \multicolumn{3}{|c|}{ Directional information } \\
\hline & & & $\begin{array}{c}\text { Turning } \\
\text { movement }\end{array}$ & Percent & Counts \\
\hline \multirow[t]{12}{*}{ U.S. 321 Intersection w/ Foothills Pkwy } & FH Pkwy 8A WB & 229 & Right Tum & $16.59 \%$ & 38 \\
\hline & & & Through & $7.42 \%$ & 17 \\
\hline & & & Left Tum & $75.98 \%$ & 174 \\
\hline & FH Pkwy 8B EB & 152 & Right Tum & $5.92 \%$ & 9 \\
\hline & & & Through & $51.32 \%$ & 78 \\
\hline & & & Left Tum & $42.76 \%$ & 65 \\
\hline & U.S. $321 \mathrm{SB}$ & 650 & Right Tum & $13.69 \%$ & 89 \\
\hline & & & Through & $83.23 \%$ & 541 \\
\hline & & & Left Turn & $3.08 \%$ & 20 \\
\hline & U.S. $321 \mathrm{NB}$ & 766 & Right Turn & $14.23 \%$ & 109 \\
\hline & & & Through & $85.12 \%$ & 652 \\
\hline & & & Left Tum & $0.65 \%$ & 5 \\
\hline \multirow[t]{6}{*}{ U.S. 321 Convergence w/ SR 32} & U.S. $321 \mathrm{~EB}$ & 663 & Right Tum & $13.12 \%$ & 87 \\
\hline & & & Left Turn & $86.88 \%$ & 576 \\
\hline & U.S. 321 SB & 431 & Right Turn & $71.23 \%$ & 307 \\
\hline & & & Through & $28.77 \%$ & 124 \\
\hline & SR $32 \mathrm{NB}$ & 69 & Through & $68.12 \%$ & 47 \\
\hline & & & Left Turn & $31.88 \%$ & 22 \\
\hline \multirow[t]{6}{*}{ U.S. 321 Intersection w/SR 416} & SR 416 SB & 289 & Right Turn & $88.24 \%$ & 255 \\
\hline & & & Left Tum & $11.76 \%$ & 34 \\
\hline & U.S. 321 WB & 982 & Right Turn & $9.98 \%$ & 98 \\
\hline & & & Through & $90.02 \%$ & 884 \\
\hline & U.S. 321 EB & 538 & Through & $69.89 \%$ & 376 \\
\hline & & & Left Turn & $30.11 \%$ & 162 \\
\hline \multirow[t]{6}{*}{ SR 416 Intersection n i $^{\prime}$ ebb Creek Rd } & Webb Cr Rd WB & 43 & Right Turn & $4.65 \%$ & 2 \\
\hline & & & Left Turn & $95.35 \%$ & 41 \\
\hline & SR $416 \mathrm{SB}$ & 72 & Through & $98.61 \%$ & 71 \\
\hline & & & Left Turn & $1.39 \%$ & 1 \\
\hline & SR $416 \mathrm{NB}$ & 85 & Right Turn & $45.88 \%$ & 39 \\
\hline & & & Through & $54.12 \%$ & 46 \\
\hline \multirow[t]{4}{*}{ SR 416 Intersection w/ Foothills Pkwy 8B/8C } & FH Pkwy 8B WB & 102 & Through & $100.00 \%$ & 102 \\
\hline & FH Pkwy 8C EB & 0 & Through & & 0 \\
\hline & SR $416 \mathrm{SB}$ & 112 & Through & $100.00 \%$ & 112 \\
\hline & SR $416 \mathrm{NB}$ & 85 & Through & $100.00 \%$ & 85 \\
\hline \multirow[t]{6}{*}{ SR 416 Intersection w/ Foothills Pkwy ramp } & FH Pkwy ramp SB & 102 & Right Turn & $100.00 \%$ & 102 \\
\hline & & & Left Turn & $0.00 \%$ & 0 \\
\hline & SR $416 \mathrm{~EB}$ & 237 & Through & $35.87 \%$ & 85 \\
\hline & & & Left Turn & $64.14 \%$ & 152 \\
\hline & SR 416 WB & 112 & Right Turn & $0.00 \%$ & 0 \\
\hline & & & Through & $100.00 \%$ & 112 \\
\hline
\end{tabular}


Table K.45. Weekday A.M. peak-hour intersection volume projections for 2026-interchange at Highway 416 (south ramp option)-8B opened prior to completion of $8 \mathrm{C}$

\begin{tabular}{|c|c|c|c|c|c|}
\hline \multirow{2}{*}{ Intersection location } & \multirow[b]{2}{*}{ Approach } & \multirow{2}{*}{$\begin{array}{l}\text { Total } \\
\text { vehicles } \\
\text { per hour }\end{array}$} & \multicolumn{3}{|c|}{ Directional information } \\
\hline & & & $\begin{array}{c}\text { Turning } \\
\text { movement }\end{array}$ & Percent & Counts \\
\hline \multirow[t]{12}{*}{ U.S. 321 Intersection w/ Foothills Pkwy } & FH Pkwy 8A WB & 300 & Right Turn & $12.00 \%$ & 36 \\
\hline & & & Through & $10.00 \%$ & 30 \\
\hline & & & Left Turn & $78.00 \%$ & 234 \\
\hline & FH Pkwy 8B EB & 152 & Right Tum & $5.92 \%$ & 9 \\
\hline & & & Through & $57.89 \%$ & 88 \\
\hline & & & Left Tum & $36.18 \%$ & 55 \\
\hline & U.S. $321 \mathrm{SB}$ & 680 & Right Turn & $11.32 \%$ & 77 \\
\hline & & & Through & $80.15 \%$ & 545 \\
\hline & & & Left Turn & $8.53 \%$ & 58 \\
\hline & U.S. $321 \mathrm{NB}$ & 849 & Right Turn & $20.49 \%$ & 174 \\
\hline & & & Through & $79.03 \%$ & 671 \\
\hline & & & Left Turn & $0.47 \%$ & 4 \\
\hline \multirow[t]{6}{*}{ U.S. 321 Convergence $w / S R 32$} & U.S. $321 \mathrm{~EB}$ & 547 & Right Turn & $12.43 \%$ & 68 \\
\hline & & & Left Turn & $87.57 \%$ & 479 \\
\hline & U.S. $321 \mathrm{SB}$ & 306 & Right Turn & $73.53 \%$ & 225 \\
\hline & & & Through & $26.47 \%$ & 81 \\
\hline & SR $32 \mathrm{NB}$ & 90 & Through & $68.89 \%$ & 62 \\
\hline & & & Left Turn & $31.11 \%$ & 28 \\
\hline \multirow[t]{6}{*}{ U.S. 321 Intersection w/ SR 416} & SR 416 SB & 305 & Right Turn & $89.18 \%$ & 272 \\
\hline & & & Left Tum & $10.82 \%$ & 33 \\
\hline & U.S. 321 WB & 975 & Right Turn & $1.85 \%$ & 18 \\
\hline & & & Through & $98.15 \%$ & 957 \\
\hline & U.S. 321 EB & 632 & Through & $67.41 \%$ & 426 \\
\hline & & & Left Tum & $32.59 \%$ & 206 \\
\hline \multirow[t]{6}{*}{ SR 416 Intersection w/ Webb Creek Rd } & Webb Cr Rd WB & 82 & Right Turn & $3.66 \%$ & 3 \\
\hline & & & Left Turn & $96.34 \%$ & 79 \\
\hline & SR 416 SB & 65 & Through & $96.92 \%$ & 63 \\
\hline & & & Left Turn & $3.08 \%$ & 2 \\
\hline & SR $416 \mathrm{NB}$ & 115 & Right Turn & $48.70 \%$ & 56 \\
\hline & & & Through & $51.30 \%$ & 59 \\
\hline \multirow[t]{4}{*}{ SR 416 Intersection w/ Foothills Pkwy 8B/8C } & FH Pkwy 8B WB & 108 & Through & $100.00 \%$ & 108 \\
\hline & FH Pkwy 8C EB & 0 & Through & & 0 \\
\hline & SR 416 SB & 142 & Through & $100.00 \%$ & 142 \\
\hline & SR $416 \mathrm{NB}$ & 115 & Through & $100.00 \%$ & 115 \\
\hline \multirow[t]{6}{*}{ SR 416 Intersection w/ Foothills Pkwy ramp } & FH Pkwy ramp SB & 108 & Right Tum & $100.00 \%$ & 108 \\
\hline & & & Left Tum & $0.00 \%$ & 0 \\
\hline & SR 416 EB & 267 & Through & $43.07 \%$ & 115 \\
\hline & & & Left Turn & $56.93 \%$ & 152 \\
\hline & SR 416 WB & 142 & Right Turn & $0.00 \%$ & 0 \\
\hline & & & Through & $100.00 \%$ & 142 \\
\hline
\end{tabular}


Table K.46. Weekday P.M. peak-hour intersection volume projections for 2026 -interchange at Highway 416 (south ramp option)-8B opened prior to completion of $8 \mathrm{C}$

\begin{tabular}{|c|c|c|c|c|c|}
\hline \multirow[b]{2}{*}{ Intersection location } & \multirow[b]{2}{*}{ Approach } & \multirow{2}{*}{$\begin{array}{c}\text { Total } \\
\text { vehicles } \\
\text { per hour }\end{array}$} & \multicolumn{3}{|c|}{ Directional information } \\
\hline & & & $\begin{array}{c}\text { Turning } \\
\text { movement }\end{array}$ & Percent & Counts \\
\hline \multirow[t]{6}{*}{ U.S. 321 Intersection w/ Foothills Pkwy } & FH Pkwy 8A WB & 221 & Right Tum & $14.93 \%$ & 33 \\
\hline & & & Through & $9.50 \%$ & 21 \\
\hline & & & Left Turn & $75.57 \%$ & 167 \\
\hline & FH Pkwy 8B EB & 201 & Right Turn & $5.97 \%$ & 12 \\
\hline & & & Through & $57.71 \%$ & 116 \\
\hline & $\cdot$ & & Left Tum & $36.32 \%$ & 73 \\
\hline & U.S. $321 \mathrm{SB}$ & 711 & Right Turn & $12.10 \%$ & 86 \\
\hline & & & Through & $85.23 \%$ & 606 \\
\hline & & & Left Turn & $2.67 \%$ & 19 \\
\hline & U.S. $321 \mathrm{NB}$ & 867 & Right Turn & $13.49 \%$ & 117 \\
\hline & & & Through & $85.93 \%$ & 745 \\
\hline - & & & Left Tum & $0.58 \%$ & 5 \\
\hline \multirow[t]{6}{*}{ U.S. 321 Convergence w/ SR 32} & U.S. $321 \mathrm{~EB}$ & 724 & Right Turn & $12.71 \%$ & 92 \\
\hline & & & Left Turn & $87.29 \%$ & 632 \\
\hline & U.S. $321 \mathrm{SB}$ & 306 & Right Turn & $74.18 \%$ & 227 \\
\hline & & & Through & $25.82 \%$ & 79 \\
\hline & SR $32 \mathrm{NB}$ & 63 & Through & $66.67 \%$ & 42 \\
\hline & & & Left Turn & $33.33 \%$ & 21 \\
\hline \multirow[t]{6}{*}{ U.S. 321 Intersection w/ SR 416} & SR 416 SB & 202 & Right Tum & $91.09 \%$ & 184 \\
\hline & & & Left Turn & $8.91 \%$ & 18 \\
\hline & U.S. 321 WB & 943 & Right Turn & $4.14 \%$ & 39 \\
\hline & & & Through & $95.86 \%$ & 904 \\
\hline & U.S. $321 \mathrm{~EB}$ & 482 & Through & $76.56 \%$ & 369 \\
\hline & & & Left Turn & $23.44 \%$ & 113 \\
\hline \multirow[t]{6}{*}{ SR 416 Intersection w/ Webb Creek Rd } & Webb Cr Rd WB & 89 & Right Turn & $6.74 \%$ & 6 \\
\hline & & & Left Tum & $93.26 \%$ & 83 \\
\hline & SR 416 SB & 80 & Through & $98.75 \%$ & 79 \\
\hline & & & Left Tum & $1.25 \%$ & 1 \\
\hline & SR 416 NB & 148 & Right Turn & $44.59 \%$ & 66 \\
\hline & & & Through & $55.41 \%$ & 82 \\
\hline \multirow[t]{4}{*}{ SR 416 Intersection w/ Foothills Pkwy $8 \mathrm{~B} / 8 \mathrm{C}$} & FH Pkwy 8B WB & 109 & Through & $100.00 \%$ & 109 \\
\hline & FH Pkwy 8C EB & 0 & Through & & 0 \\
\hline & SR 416 SB & 163 & Through & $100.00 \%$ & 163 \\
\hline & SR $416 \mathrm{NB}$ & 149 & Through & $100.00 \%$ & 149 \\
\hline \multirow[t]{6}{*}{ SR 416 Intersection w/ Foothills Pkwy ramp } & FH Pkwy ramp SB & 109 & Right Turn & $100.00 \%$ & 109 \\
\hline & & & Left Turn & $0.00 \%$ & 0 \\
\hline & SR $416 \mathrm{~EB}$ & 349 & Through & $42.69 \%$ & 149 \\
\hline & & & Left Tum & $57.31 \%$ & 200 \\
\hline & SR 416 WB & 163 & Right Turn & $0.00 \%$ & 0 \\
\hline & & & Through & $100.00 \%$ & 163 \\
\hline
\end{tabular}


Table K.47. Weekend A.M. peak-hour intersection volume projections for 2026-interchange at Highway 416 (south ramp option)-8B opened prior to completion of 8C

\begin{tabular}{|c|c|c|c|c|c|}
\hline \multirow[b]{2}{*}{ Intersection location } & \multirow[b]{2}{*}{ Approach } & \multirow{2}{*}{$\begin{array}{c}\text { Total } \\
\text { vehicles } \\
\text { per hour }\end{array}$} & \multicolumn{3}{|c|}{ Directional information } \\
\hline & & & $\begin{array}{l}\text { Turning } \\
\text { movement }\end{array}$ & Percent & Counts \\
\hline \multirow[t]{3}{*}{ U.S. 321 Intersection w/ Foothills Pkwy } & \multirow[t]{3}{*}{ FH Pkwy 8A WB } & \multirow[t]{3}{*}{507} & Right Turn & $12.03 \%$ & 61 \\
\hline & & & Through & $9.86 \%$ & 50 \\
\hline & & & Left Tum & $78.11 \%$ & 396 \\
\hline \multirow{9}{*}{ · } & \multirow[t]{3}{*}{ FH Pkwy 8B EB } & \multirow[t]{3}{*}{208} & Right Turn & $5.77 \%$ & 12 \\
\hline & & & Through & $57.69 \%$ & 120 \\
\hline & & & Left Turn & $36.54 \%$ & 76 \\
\hline & \multirow[t]{3}{*}{ U.S. $321 \mathrm{SB}$} & \multirow[t]{3}{*}{749} & Right Turn & $11.35 \%$ & 85 \\
\hline & & & Through & $79.97 \%$ & 599 \\
\hline & & & Left Tum & $8.68 \%$ & 65 \\
\hline & \multirow[t]{3}{*}{ U.S. $321 \mathrm{NB}$} & \multirow[t]{3}{*}{1046} & Right Tum & $20.65 \%$ & 216 \\
\hline & & & Through & $78.87 \%$ & 825 \\
\hline & & & Left Turn & $0.48 \%$ & 5 \\
\hline \multirow[t]{6}{*}{ U.S. 321 Convergence w/ SR 32} & \multirow[t]{2}{*}{ U.S. 321 EB } & \multirow[t]{2}{*}{749} & Right Tum & $12.42 \%$ & 93 \\
\hline & & & Left Tum & $87.58 \%$ & 656 \\
\hline & \multirow[t]{2}{*}{ U.S. 321 SB } & \multirow[t]{2}{*}{696} & Right Turn & $73.56 \%$ & 512 \\
\hline & & & Through & $26.44 \%$ & 184 \\
\hline & \multirow[t]{2}{*}{ SR 32 NB } & \multirow[t]{2}{*}{123} & Through & $69.92 \%$ & 86 \\
\hline & & & Left Turn & $30.08 \%$ & 37 \\
\hline \multirow{6}{*}{ U.S. 321 Intersection w/ SR 416} & \multirow[t]{2}{*}{ STR 416 SB } & \multirow[t]{2}{*}{331} & Right Turn & $89.43 \%$ & 296 \\
\hline & & & Left Tum & $10.57 \%$ & 35 \\
\hline & \multirow{2}{*}{ U.S. 321 WB } & 1184 & Right Tum & $194 \%$ & 23 \\
\hline & & & Through & $98.06 \%$ & 1161 \\
\hline & U.S. 321 EB & 806 & Through & $67.12 \%$ & 541 \\
\hline & & & Left Turn & $32.88 \%$ & 265 \\
\hline SR 416 Intersection w/ Webb Creek Rd & Webb Cr Rd WB & 52 & Right Turn & $3.85 \%$ & 2 \\
\hline & & & Left Tum & $96.15 \%$ & 50 \\
\hline & SR 416 SB & 48 & Through & $95.83 \%$ & 46 \\
\hline & & & Left Turn & $4.17 \%$ & 2 \\
\hline & SR $416 \mathrm{NB}$ & 96 & Right Tum & $47.92 \%$ & 46 \\
\hline & & & Through & $52.08 \%$ & 50 \\
\hline SR 416 Intersection w/ Foothills Pkwy 8B/8C & FH Pkwy 8B WB & 140 & Through & $100.00 \%$ & 140 \\
\hline & FH Pkwy 8C EB & 0 & Through & & 0 \\
\hline & SR 416 SB & 96 & Through & $100.00 \%$ & 96 \\
\hline . & SR $416 \mathrm{NB}$ & 96 & Through & $100.00 \%$ & 96 \\
\hline SR 416 Intersection w/ Foothills Pkwy ramp & FH Pkwy ramp SB & 140 & Right Tum & $100.00 \%$ & 140 \\
\hline & & & Left Turn & $0.00 \%$ & 0 \\
\hline & SR 416 EB & 304 & Through & $31.58 \%$ & 96 \\
\hline & & & Left Turn & $68.42 \%$ & 208 \\
\hline . & SR 416 WB & 96 & Right Tum & $0.00 \%$ & 0 \\
\hline & & & Through & $100.00 \%$ & 96 \\
\hline
\end{tabular}


Table K.48. Weekend P.M. peak-hour intersection volume projections for 2026-interchange at Highway 416 (south ramp option) $-8 \mathrm{~B}$ opened prior to completion of $8 \mathrm{C}$

\begin{tabular}{|c|c|c|c|c|c|}
\hline \multirow[b]{2}{*}{ Intersection location } & \multirow[b]{2}{*}{ Approach } & \multirow{2}{*}{$\begin{array}{c}\text { Total } \\
\text { vehicles } \\
\text { per hour }\end{array}$} & \multicolumn{3}{|c|}{ Directional information } \\
\hline & & & $\begin{array}{l}\text { Turning } \\
\text { movement }\end{array}$ & Percent & Counts \\
\hline \multirow[t]{12}{*}{ U.S. 321 Intersection w/ Foothills Pkwy } & FH Pkwy 8A WB & 308 & Right Tum & $15.26 \%$ & 47 \\
\hline & & & Through & $9.42 \%$ & 29 \\
\hline & & & Left Tum & $75.32 \%$ & 232 \\
\hline & FH Pkwy 8B EB & 241 & Right Tum & $5.81 \%$ & 14 \\
\hline & & & Through & $57.68 \%$ & 139 \\
\hline & & & Left Tum & $36.51 \%$ & 88 \\
\hline & U.S. $321 \mathrm{SB}$ & 833 & Right Tum & $12.12 \%$ & 101 \\
\hline & & & Through & $85.23 \%$ & 710 \\
\hline & & & Left Turn & $2.64 \%$ & 22 \\
\hline & U.S. $321 \mathrm{NB}$ & 991 & Right Tum & $13.42 \%$ & 133 \\
\hline & & & Through & $86.07 \%$ & 853 \\
\hline & & & Left Turn & $0.50 \%$ & 5 \\
\hline \multirow[t]{6}{*}{ U.S. 321 Convergence w/ SR 32} & U.S. $321 \mathrm{~EB}$ & 871 & Right Turn & $12.74 \%$ & 111 \\
\hline & & & Left Turn & $87.26 \%$ & 760 \\
\hline & U.S. $321 \mathrm{SB}$ & 580 & Right Turn & $73.97 \%$ & 429 \\
\hline & & & Through & $26.03 \%$ & 151 \\
\hline & SR $32 \mathrm{NB}$ & 82 & Through & $65.85 \%$ & 54 \\
\hline & & & Left Tum & $34.15 \%$ & 28 \\
\hline \multirow[t]{6}{*}{ U.S. 321 Intersection w/ SR 416} & SR 416 SB & 391 & Right Turn & $90.54 \%$ & 354 \\
\hline & & & Left Turn & $9.46 \%$ & 37 \\
\hline & U.S. 321 WB & 1262 & Right Turn & $4.20 \%$ & 53 \\
\hline & & & Through & $95.80 \%$ & 1209 \\
\hline & U.S. $321 \mathrm{~EB}$ & 683 & Through & $77.01 \%$ & 526 \\
\hline & & & Left Turn & $22.99 \%$ & 157 \\
\hline \multirow[t]{6}{*}{ SR 416 Intersection w/ Webb Creek Rd } & Webb Cr Rd WB & 68 & Right Turn & $7.35 \%$ & 5 \\
\hline & & & Left Turn & $92.65 \%$ & 63 \\
\hline & SR 416 SB & 110 & Through & $99.09 \%$ & 109 \\
\hline & & & Left Turn & $0.91 \%$ & 1 \\
\hline & SR $416 \mathrm{NB}$ & 148 & Right Tum & $44.59 \%$ & 66 \\
\hline & & & Through & $55.41 \%$ & 82 \\
\hline \multirow[t]{4}{*}{ SR 416 Intersection w/ Foothills Pkwy 8B/8C } & FH Pkwy 8B WB & 131 & Through & $100.00 \%$ & 131 \\
\hline & FH Pkwy 8C EB & 0 & Through & & 0 \\
\hline & SR 416 SB & 171 & Through & $100.00 \%$ & 171 \\
\hline & SR $416 \mathrm{NB}$ & 149 & Through & $100.00 \%$ & 149 \\
\hline \multirow[t]{6}{*}{ SR 416 Intersection w/ Foothills Pkwy ramp } & FH Pkwy ramp SB & 131 & Right Tum & $100.00 \%$ & 131 \\
\hline & & & Left Tum & $0.00 \%$ & 0 \\
\hline & SR $416 \mathrm{~EB}$ & 390 & Through & $38.21 \%$ & 149 \\
\hline & & & Left Turn & $61.79 \%$ & 241 \\
\hline & SR 416 WB & 171 & Right Turn & $0.00 \%$ & 0 \\
\hline & & & Through & $100.00 \%$ & 171 \\
\hline
\end{tabular}


Table K.49. Weekday A.M. peak-hour intersection volume projections for 2006-interchange at U.S. 321 (both options)-8B opened prior to completion of 8C

\begin{tabular}{|c|c|c|c|c|c|}
\hline \multirow[b]{2}{*}{ Intersection location } & \multirow[b]{2}{*}{ Approach } & \multirow{2}{*}{$\begin{array}{c}\text { Total } \\
\text { vehicles } \\
\text { per hour }\end{array}$} & \multicolumn{3}{|c|}{ Directional information } \\
\hline & & & $\begin{array}{c}\text { Turning } \\
\text { movement }\end{array}$ & Percent & Counts \\
\hline \multirow[t]{4}{*}{ U.S. 321 Intersection w/ Foothills Pkwy } & FH Pkwy 8A WB & 222 & $\begin{array}{l}\text { Right Turn } \\
\text { Through } \\
\text { Left Tumn }\end{array}$ & $\begin{array}{r}13.06 \% \\
7.66 \% \\
79.28 \%\end{array}$ & $\begin{array}{r}29 \\
17 \\
176\end{array}$ \\
\hline & FH Pkwy 8B EB & 96 & $\begin{array}{l}\text { Right Turn } \\
\text { Through } \\
\text { Left Turn }\end{array}$ & $\begin{array}{r}6.25 \% \\
51.04 \% \\
42.71 \%\end{array}$ & $\begin{array}{r}6 \\
49 \\
41\end{array}$ \\
\hline & U.S. 321 SB & 536 & $\begin{array}{l}\text { Right Turn } \\
\text { Through } \\
\text { Left Turn }\end{array}$ & $\begin{array}{r}12.69 \% \\
77.43 \% \\
9.89 \%\end{array}$ & $\begin{array}{r}68 \\
415 \\
53\end{array}$ \\
\hline & U.S. $321 \mathrm{NB}$ & 660 & $\begin{array}{l}\text { Right Turn } \\
\text { Through } \\
\text { Left Tum }\end{array}$ & $\begin{array}{r}21.67 \% \\
77.73 \% \\
0.61 \%\end{array}$ & $\begin{array}{r}143 \\
513 \\
4\end{array}$ \\
\hline \multirow[t]{3}{*}{ U.S. 321 Convergence w/ SR 32} & U.S. 321 EB & 416 & $\begin{array}{l}\text { Right Tum } \\
\text { Left Turn }\end{array}$ & $\begin{array}{l}12.74 \% \\
87.26 \%\end{array}$ & $\begin{array}{r}53 \\
363\end{array}$ \\
\hline & U.S. $321 \mathrm{SB}$ & 227 & $\begin{array}{l}\text { Right Turn } \\
\text { Through }\end{array}$ & $\begin{array}{l}70.93 \% \\
29.07 \%\end{array}$ & $\begin{array}{r}161 \\
66\end{array}$ \\
\hline & SR $32 \mathrm{NB}$ & 76 & $\begin{array}{l}\text { Through } \\
\text { Left Turn }\end{array}$ & $\begin{array}{l}71.05 \% \\
28.95 \%\end{array}$ & $\begin{array}{l}54 \\
22\end{array}$ \\
\hline \multirow[t]{3}{*}{ U.S. 321 Intersection w/ SR 416} & SR 416 SB & 125 & $\begin{array}{l}\text { Right Tum } \\
\text { Left Tum }\end{array}$ & $\begin{array}{l}76.00 \% \\
24.00 \%\end{array}$ & $\begin{array}{l}95 \\
30\end{array}$ \\
\hline & U.S. $321 \mathrm{WB}$ & 824 & $\begin{array}{l}\text { Right Turn } \\
\text { Through }\end{array}$ & $\begin{array}{r}4.13 \% \\
95.87 \%\end{array}$ & $\begin{array}{r}34 \\
790\end{array}$ \\
\hline & U.S. 321 EB & 519 & $\begin{array}{l}\text { Through } \\
\text { Left Tum }\end{array}$ & $\begin{array}{l}78.61 \% \\
21.39 \%\end{array}$ & $\begin{array}{l}408 \\
111\end{array}$ \\
\hline \multirow[t]{3}{*}{ SR 416 Intersection w/ Webb Creek Rd } & Webb Cr Rd WB & 54 & $\begin{array}{l}\text { Right Turn } \\
\text { Left Turn }\end{array}$ & $\begin{array}{r}3.70 \% \\
96.30 \%\end{array}$ & $\begin{array}{r}2 \\
52\end{array}$ \\
\hline & SR $416 \mathrm{SB}$ & 42 & $\begin{array}{l}\text { Through } \\
\text { Left Tum }\end{array}$ & $\begin{array}{r}97.62 \% \\
2.38 \%\end{array}$ & $\begin{array}{r}41 \\
1\end{array}$ \\
\hline & SR 416 NB & 66 & $\begin{array}{l}\text { Right Tum } \\
\text { Through }\end{array}$ & $\begin{array}{l}50.00 \% \\
50.00 \%\end{array}$ & $\begin{array}{l}33 \\
33\end{array}$ \\
\hline \multirow[t]{4}{*}{ SR 416 Intersection w/ Foothills Pkwy $8 \mathrm{~B} / 8 \mathrm{C}$} & FH Pkwy 8B WB & 0 & $\begin{array}{l}\text { Right Turn } \\
\text { Through } \\
\text { Left Turn }\end{array}$ & · & $\begin{array}{l}0 \\
0 \\
0\end{array}$ \\
\hline & FH Pkwy 8C EB & 0 & $\begin{array}{l}\text { Right Turn } \\
\text { Through } \\
\text { Left Tum }\end{array}$ & & $\begin{array}{l}0 \\
0 \\
0\end{array}$ \\
\hline & SR $416 \mathrm{SB}$ & 93 & $\begin{array}{l}\text { Right Turn } \\
\text { Through } \\
\text { Left Turn }\end{array}$ & $\begin{array}{r}0.00 \% \\
100.00 \% \\
0.00 \%\end{array}$ & $\begin{array}{r}0 \\
93 \\
0\end{array}$ \\
\hline & SR $416 \mathrm{NB}$ & 66 & $\begin{array}{l}\text { Right Tum } \\
\text { Through } \\
\text { Left Turn }\end{array}$ & $\begin{array}{r}0.00 \% \\
100.00 \% \\
0.00 \%\end{array}$ & $\begin{array}{r}0 \\
66 \\
0\end{array}$ \\
\hline
\end{tabular}


Table K.49. Weekday A.M. peak-hour intersection volume projections for 2006-interchange at U.S. 321 (directly connected)-8B opened prior to $8 \mathrm{C}$ completion (continued)

\begin{tabular}{|c|c|c|c|c|c|}
\hline \multirow[b]{2}{*}{ Intersection location } & \multirow[b]{2}{*}{ Approach } & \multirow{2}{*}{$\begin{array}{c}\text { Total } \\
\text { vehicles } \\
\text { per hour }\end{array}$} & \multicolumn{3}{|c|}{ Directional information } \\
\hline & & & $\begin{array}{c}\text { Turning } \\
\text { movement }\end{array}$ & Percent & Counts \\
\hline \multirow[t]{3}{*}{ U.S. 321 Intersection w/ Foothills Pkwy 8B } & FH Pkwy 8B & 139 & $\begin{array}{l}\text { Right Turn } \\
\text { Left Turn }\end{array}$ & $\begin{array}{r}100.00 \% \\
0.00 \%\end{array}$ & $\begin{array}{r}139 \\
0\end{array}$ \\
\hline & U.S. 321 WB & 735 & $\begin{array}{l}\text { Right Turn } \\
\text { Through }\end{array}$ & $\begin{array}{r}0.00 \% \\
100.00 \%\end{array}$ & $\begin{array}{r}0 \\
735\end{array}$ \\
\hline & U.S. $321 \mathrm{~EB}$ & 426 & $\begin{array}{l}\text { Through } \\
\text { Left Turn }\end{array}$ & $\begin{array}{l}77.46 \% \\
22.54 \%\end{array}$ & $\begin{array}{r}330 \\
96\end{array}$ \\
\hline
\end{tabular}

Table K.49. Weekday A.M. peak-hour intersection volume projections for 2006-interchange at U.S. 321 (Webb Creek Rd. connection)-8B opened prior to 8C completion (continued)

\begin{tabular}{|c|c|c|c|c|c|}
\hline \multirow[b]{2}{*}{ Intersection location } & \multirow[b]{2}{*}{ Approach } & \multirow{2}{*}{$\begin{array}{c}\text { Total } \\
\text { vehicles } \\
\text { per hour }\end{array}$} & \multicolumn{3}{|c|}{ Directional information } \\
\hline & & & $\begin{array}{c}\text { Turning } \\
\text { movement }\end{array}$ & Percent & Counts \\
\hline \multirow[t]{3}{*}{ Foothills Pkwy 8B/Webb Creek Rd to US 321} & FH Pkwy 8B & 183 & $\begin{array}{l}\text { Right Turn } \\
\text { Left Turn }\end{array}$ & $\begin{array}{l}87.98 \% \\
12.02 \%\end{array}$ & $\begin{array}{r}161 \\
22\end{array}$ \\
\hline & U.S. $321 \mathrm{WB}$ & 757 & $\begin{array}{l}\text { Right Turn } \\
\text { Through }\end{array}$ & $\begin{array}{r}2.91 \% \\
97.09 \%\end{array}$ & $\begin{array}{r}22 \\
735\end{array}$ \\
\hline & U.S. $321 \mathrm{~EB}$ & 448 & $\begin{array}{l}\text { Through } \\
\text { Left Tum }\end{array}$ & $\begin{array}{l}73.66 \% \\
26.34 \%\end{array}$ & $\begin{array}{l}330 \\
118\end{array}$ \\
\hline
\end{tabular}


Table K.50. Weekday P.M. peak-hour intersection volume projections for 2006 -interchange at U.S. 321 (both options)-8B opened prior to completion of $8 \mathrm{C}$

\begin{tabular}{|c|c|c|c|c|c|}
\hline \multirow{2}{*}{ Intersection location } & \multirow[b]{2}{*}{ Approach } & \multirow{2}{*}{$\begin{array}{c}\text { Total } \\
\text { vehicles } \\
\text { per hour }\end{array}$} & \multicolumn{3}{|c|}{ Directional information } \\
\hline & & & $\begin{array}{c}\text { Tuming } \\
\text { movement }\end{array}$ & Percent & Counts \\
\hline \multirow[t]{12}{*}{ U.S. 321 Intersection w/ Foothills Pkwy } & FH Pkwy 8A WB & 163 & Right Turn & $15.95 \%$ & 26 \\
\hline & & & Through & $7.36 \%$ & 12 \\
\hline & & & Left Turn & $76.69 \%$ & 125 \\
\hline & FH Pkwy 8B EB & 127 & Right Turn & $6.30 \%$ & 8 \\
\hline & & & Through & $51.18 \%$ & 65 \\
\hline & & & Left Turn & $42.52 \%$ & 54 \\
\hline & U.S. $321 \mathrm{SB}$ & 554 & Right Tum & $13.72 \%$ & 76 \\
\hline & 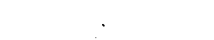 & & Through & $83.21 \%$ & 461 \\
\hline & & & Left Turn & $3.07 \%$ & 17 \\
\hline & U.S. $321 \mathrm{NB}$ & 671 & Right Turn & $14.31 \%$ & 96 \\
\hline & & & Through & $84.95 \%$ & 570 \\
\hline & & & Left Tum & $0.75 \%$ & 5 \\
\hline \multirow{6}{*}{ U.S. 321 Convergence w/ SR 32} & U.S. $321 \mathrm{~EB}$ & 551 & Right Tum & $13.07 \%$ & 72 \\
\hline & & & Left Tum & $86.93 \%$ & 479 \\
\hline & U.S. $321 \mathrm{SB}$ & 228 & Right Turn & $71.49 \%$ & 163 \\
\hline & & & Through & $28.51 \%$ & 65 \\
\hline & SR 32 NB & 54 & Through & $68.52 \%$ & 37 \\
\hline & & & Left Turn & $31.48 \%$ & 17 \\
\hline \multirow{6}{*}{ U.S. 321 Intersection w/ SR 416} & SR $416 \mathrm{SB}$ & 82 & Right Turn & $79.27 \%$ & 65 \\
\hline & & & Left Turn & $20.73 \%$ & 17 \\
\hline & U.S. 321 WB & 820 & Right Tum & $9.02 \%$ & 74 \\
\hline & & & Through & $90.98 \%$ & 746 \\
\hline & U.S. $321 \mathrm{~EB}$ & 415 & Through & $85.30 \%$ & 354 \\
\hline & & & Left Turn & $14.70 \%$ & 61 \\
\hline \multirow[t]{6}{*}{ SR 416 Intersection w/ Webb Creek Rd } & Webb Cr Rd WB & 58 & Right Turn & $5.17 \%$ & 3 \\
\hline & & & Left Turn & $94.83 \%$ & 55 \\
\hline & SR $416 \mathrm{SB}$ & 53 & Through & $98.11 \%$ & 52 \\
\hline & & & Left Turn & $1.89 \%$ & 1 \\
\hline & SR 416 NB & 85 & Right Turn & $45.88 \%$ & 39 \\
\hline & & & Through & $54.12 \%$ & 46 \\
\hline \multirow[t]{12}{*}{ SR 416 Intersection w/ Foothills Pkwy 8B/8C } & FH Pkwy 8B WB & 0 & Right Turn & & 0 \\
\hline & & & Through & & 0 \\
\hline & & & Left Tum & & 0 \\
\hline & FH Pkwy 8C EB & 0 & Right Turn & & 0 \\
\hline & & & Through & & $\mathbf{0}$ \\
\hline & & & Left Turn & & 0 \\
\hline & SR $416 \mathrm{SB}$ & 107 & Right Tum & $0.00 \%$ & 0 \\
\hline & & & Through & $100.00 \%$ & 107 \\
\hline & & & Left Turn & $0.00 \%$ & 0 \\
\hline & SR $416 \mathrm{NB}$ & 85 & Right Tum & $0.00 \%$ & 0 \\
\hline & & & Through & $100.00 \%$ & 85 \\
\hline & & & Left Turn & $0.00 \%$ & 0 \\
\hline
\end{tabular}


Table K.50. Weekday P.M. peak-hour intersection volume projections for 2006-interchange at U.S. 321 (directly connected) $-8 \mathrm{~B}$ opened prior to $8 \mathrm{C}$ completion (continued)

\begin{tabular}{|c|c|c|c|c|c|}
\hline \multirow[b]{2}{*}{ Intersection location } & \multirow[b]{2}{*}{ Approach } & \multirow{2}{*}{$\begin{array}{c}\text { Total } \\
\text { vehicles } \\
\text { per hour }\end{array}$} & \multicolumn{3}{|c|}{ Directional information } \\
\hline & & & $\begin{array}{l}\text { Turning } \\
\text { movement }\end{array}$ & Percent & Counts \\
\hline \multirow[t]{3}{*}{ U.S. 321 Intersection w/ Foothills Pkwy 8B } & FH Pkwy 8B & 183. & $\begin{array}{l}\text { Right Turn } \\
\text { Left Turn }\end{array}$ & $\begin{array}{r}100.00 \% \\
0.00 \%\end{array}$ & $\begin{array}{r}183 \\
0\end{array}$ \\
\hline & U.S. $321 \mathrm{WB}$ & 695 & $\begin{array}{l}\text { Right Tum } \\
\text { Through }\end{array}$ & $\begin{array}{r}0.00 \% \\
100.00 \%\end{array}$ & $\begin{array}{r}0 \\
695\end{array}$ \\
\hline & U.S. $321 \mathrm{~EB}$ & 412 & $\begin{array}{l}\text { Through } \\
\text { Left Turn }\end{array}$ & $\begin{array}{l}69.42 \% \\
30.58 \%\end{array}$ & $\begin{array}{l}286 \\
126\end{array}$ \\
\hline
\end{tabular}

Table K.50. Weekday P.M. peak-hour intersection volume projections for 2006-interchange at U.S. 321 (Webb Creek Rd. connection)-8B opened prior to $8 \mathrm{C}$ completion (continued)

\begin{tabular}{|c|c|c|c|c|c|}
\hline \multirow[b]{2}{*}{ Intersection location } & \multirow[b]{2}{*}{ Approach } & \multirow{2}{*}{$\begin{array}{c}\text { Total } \\
\text { vehicles } \\
\text { per hour }\end{array}$} & \multicolumn{3}{|c|}{ Directional information } \\
\hline & & & $\begin{array}{c}\text { Turning } \\
\text { movement }\end{array}$ & Percent & Counts \\
\hline \multirow[t]{3}{*}{ Foothills Pkwy 8B/Webb Creek Rd to US 321} & FH Pkwy 8B & 233 & $\begin{array}{l}\text { Right Turn } \\
\text { Left Turn }\end{array}$ & $\begin{array}{l}89.27 \% \\
10.73 \%\end{array}$ & $\begin{array}{r}208 \\
25\end{array}$ \\
\hline & U.S. $321 \mathrm{WB}$ & 720 & $\begin{array}{l}\text { Right Turn } \\
\text { Through }\end{array}$ & $\begin{array}{r}3.47 \% \\
96.53 \%\end{array}$ & $\begin{array}{r}25 \\
695\end{array}$ \\
\hline & U.S. $321 \mathrm{~EB}$ & 437 & $\begin{array}{l}\text { Through } \\
\text { Left Tum }\end{array}$ & $\begin{array}{l}65.45 \% \\
34.55 \%\end{array}$ & $\begin{array}{l}286 \\
151\end{array}$ \\
\hline
\end{tabular}


Table K.51. Weekend A.M. peak-hour intersection volume projections for 2006-interchange at U.S. 321 (both options)-8B opened prior to completion of $8 \mathrm{C}$

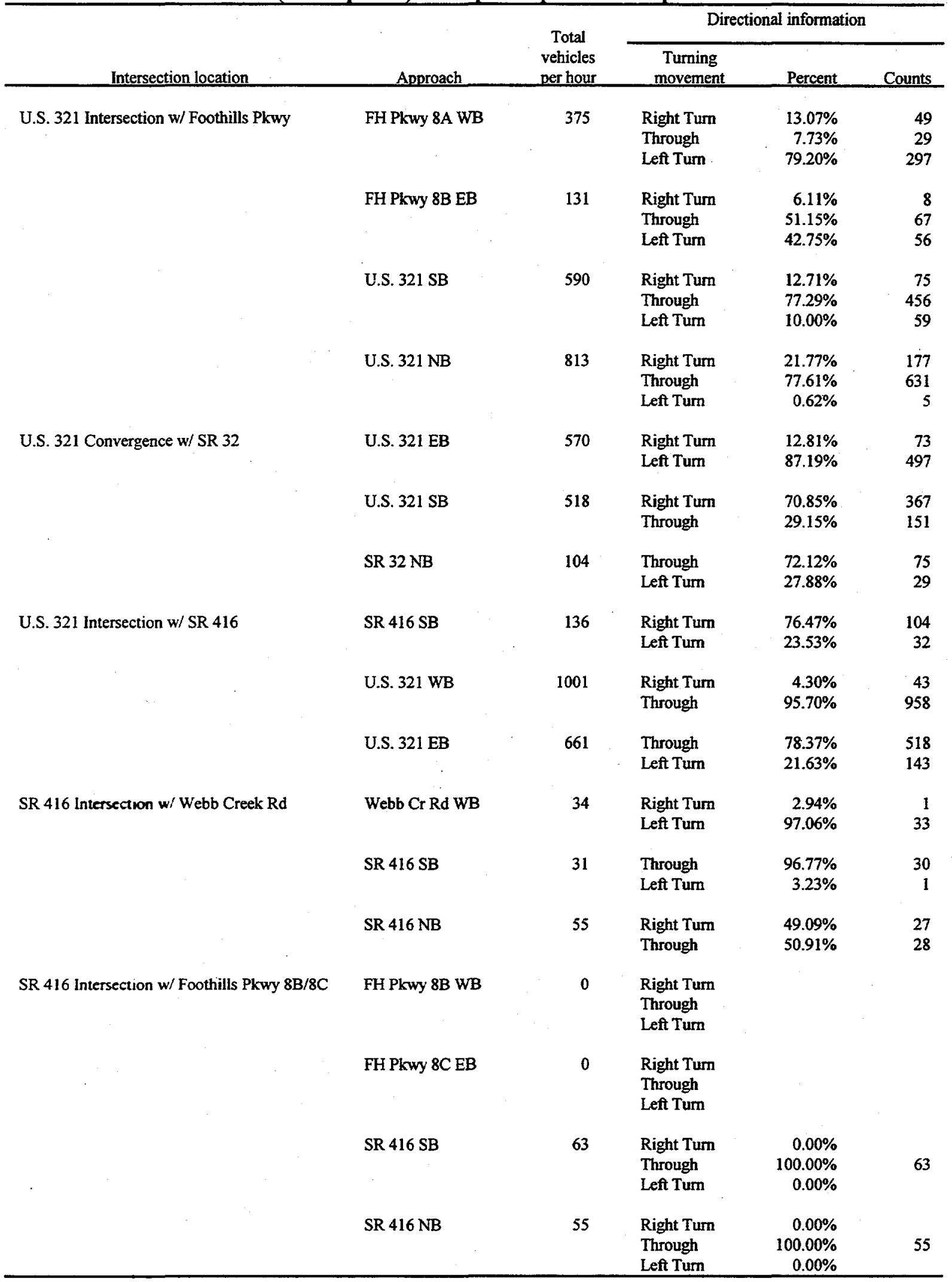


Table K.51. Weekend A.M. peak-hour intersection volume projections for 2006-interchange at U.S. 321 (directly connected) $-8 \mathrm{~B}$ opened prior to $8 \mathrm{C}$ completion (continued)

\begin{tabular}{|c|c|c|c|c|c|}
\hline \multirow[b]{2}{*}{ Intersection location } & \multirow[b]{2}{*}{ Approach } & \multirow{2}{*}{$\begin{array}{c}\text { Total } \\
\text { vehicles } \\
\text { per hour }\end{array}$} & \multicolumn{3}{|c|}{ Directional information } \\
\hline & & & $\begin{array}{c}\text { Turning } \\
\text { movement }\end{array}$ & Percent & Counts \\
\hline \multirow[t]{3}{*}{ U.S. 321 Intersection w/ Foothills Pkwy 8B } & FH Pkwy 8B & 190 & $\begin{array}{l}\text { Right Tum } \\
\text { Left Turn }\end{array}$ & $\begin{array}{r}100.00 \% \\
0.00 \%\end{array}$ & $\begin{array}{r}190 \\
0\end{array}$ \\
\hline & U.S. $321 \mathrm{WB}$ & 892 & $\begin{array}{l}\text { Right Turn } \\
\text { Through }\end{array}$ & $\begin{array}{r}0.00 \% \\
100.00 \%\end{array}$ & $\begin{array}{r}0 \\
892\end{array}$ \\
\hline & U.S. $321 \mathrm{~EB}$ & 550 & $\begin{array}{l}\text { Through } \\
\text { Left Turn }\end{array}$ & $\begin{array}{l}76.18 \% \\
23.82 \%\end{array}$ & $\begin{array}{l}419 \\
131\end{array}$ \\
\hline
\end{tabular}

Table K.51. Weekend A.M. peak-hour intersection volume projections for 2006 -interchange at U.S. 321 (Webb Creek Rd. connection)-8B opened prior to 8C completion (continued)

\begin{tabular}{|c|c|c|c|c|c|}
\hline \multirow[b]{2}{*}{ Intersection location } & \multirow[b]{2}{*}{ Approach } & \multirow{2}{*}{$\begin{array}{c}\text { Total } \\
\text { vehicles } \\
\text { per hour }\end{array}$} & \multicolumn{3}{|c|}{ Directional information } \\
\hline & & & $\begin{array}{c}\text { Tuming } \\
\text { movement }\end{array}$ & Percent & Counts \\
\hline \multirow[t]{3}{*}{ Foothills Pkwy 8B/Webb Creek Rd to US 321} & FH Pkwy 8B & 220 & $\begin{array}{l}\text { Right Turn } \\
\text { Left Turn }\end{array}$ & $\begin{array}{r}93.18 \% \\
6.82 \%\end{array}$ & $\begin{array}{r}205 \\
15\end{array}$ \\
\hline & U.S. 321 WB & 907 & $\begin{array}{l}\text { Right Turn } \\
\text { Through }\end{array}$ & $\begin{array}{r}1.65 \% \\
98.35 \%\end{array}$ & $\begin{array}{r}15 \\
892\end{array}$ \\
\hline & U.S. $321 \mathrm{~EB}$ & 565 & $\begin{array}{l}\text { Through } \\
\text { Left Turn }\end{array}$ & $\begin{array}{l}74.16 \% \\
.25 .84 \% \\
\end{array}$ & $\begin{array}{l}419 \\
146\end{array}$ \\
\hline
\end{tabular}


Table K.52. Weekend P.M. peak-hour intersection volume projections for 2006-interchange at U.S. 321 (both options)-8B opened prior to completion of $8 \mathrm{C}$

\begin{tabular}{|c|c|c|c|c|c|}
\hline \multirow[b]{2}{*}{ Intersection location } & \multirow[b]{2}{*}{ Approach } & \multirow{2}{*}{$\begin{array}{c}\text { Total } \\
\text { vehicles } \\
\text { per hour }\end{array}$} & \multicolumn{3}{|c|}{ Directional information } \\
\hline & & & $\begin{array}{c}\text { Turning } \\
\text { movement }\end{array}$ & Percent & Counts \\
\hline \multirow[t]{12}{*}{ U.S. 321 Intersection w/ Foothills Pkwy } & FH Pkwy 8A WB & 229 & Right Tum & $16.59 \%$ & 38 \\
\hline & & & Through & $7.42 \%$ & 17 \\
\hline & & & Left Turn & $75.98 \%$ & 174 \\
\hline & FH Pkwy 8B EB & 152 & Right Tum & $5.92 \%$ & 9 \\
\hline & & & Through & $51.32 \%$ & 78 \\
\hline & & & Left Turn & $42.76 \%$ & 65 \\
\hline & U.S. 321 SB & 650 & Right Tum & $13.69 \%$ & 89 \\
\hline & & & Through & $83.23 \%$ & 541 \\
\hline & & & Left Tum & $3.08 \%$ & 20 \\
\hline & U.S. $321 \mathrm{NB}$ & 766 & Right Tum & $14.23 \%$ & 109 \\
\hline & & . & Through & $85.12 \%$ & 652 \\
\hline & & & Left Turn & $0.65 \%$ & 5 \\
\hline \multirow[t]{6}{*}{ U.S. 321 Convergence w/ SR 32} & U.S. $321 \mathrm{~EB}$ & 663 & Right Turn & $13.12 \%$ & 87 \\
\hline & & & Left Turn & $86.88 \%$ & 576 \\
\hline & U.S. $321 \mathrm{SB}$ & 431 & Right Tum & $71.23 \%$ & 307 \\
\hline & & & Through & $28.77 \%$ & 124 \\
\hline & SR 32 NB & 69 & Through & $68.12 \%$ & 47 \\
\hline & & & Left Tum & $31.88 \%$ & 22 \\
\hline \multirow[t]{6}{*}{ U.S. 321 Intersection w/ SR 416} & SR 416 SB & 158 & Right Tum & $78.48 \%$ & 124 \\
\hline & & & Left Tum & $21.52 \%$ & 34 \\
\hline & U.S. 321 WB & 1096 & Right Turn & $8.94 \%$ & 98 \\
\hline & & & Through & $91.06 \%$ & 998 \\
\hline & U.S. $321 \mathrm{~EB}$ & 589 & Through & $85.57 \%$ & 504 \\
\hline & & & Left Tum & $14.43 \%$ & 85 \\
\hline \multirow[t]{6}{*}{ SR 416 Intersection w/ Webb Creek Rd } & Webb Cr Rd WB & 43 & Right Turn & $4.65 \%$ & 2 \\
\hline & & & Left Tum & $95.35 \%$ & 41 \\
\hline & SR 416 SB & 72 & Through & $98.61 \%$ & 71 \\
\hline & & & Left Tum & $1.39 \%$ & 1 \\
\hline & SR 416 NB & 85 & Right Turn & $45.88 \%$ & 39 \\
\hline & & & Through & $54.12 \%$ & 46 \\
\hline \multirow[t]{12}{*}{ SR 416 Intersection w/ Foothills Pkwy 8B/8C } & FH Pkwy 8B WB & 0 & Right Turn & & 0 \\
\hline & & & Through & & 0 \\
\hline & & & Left Tum & & 0 \\
\hline & FH Pkwy 8C EB & 0 & Right Turn & & 0 \\
\hline & & & Through & & 0 \\
\hline & & & Left Turn & & 0 \\
\hline & SR 416 SB & 112 & Right Turn & $0.00 \%$ & 0 \\
\hline & & & Through & $100.00 \%$ & 112 \\
\hline & & & Left Tum & $0.00 \%$ & 0 \\
\hline & SR 416 NB & 85 & Right Tum & $0.00 \%$ & 0 \\
\hline & & & Through & $100.00 \%$ & 85 \\
\hline & & & Left Turn & $0.00 \%$ & 0 \\
\hline
\end{tabular}


Table K.52. Weekend P.M. peak-hour intersection volume projections for 2006-interchange at U.S. 321 (directly connected) $-8 \mathrm{~B}$ opened prior to $8 \mathrm{C}$ completion (continued)

\begin{tabular}{|c|c|c|c|c|c|}
\hline \multirow[b]{2}{*}{ Intersection location } & \multirow[b]{2}{*}{ Approach } & \multirow{2}{*}{$\begin{array}{c}\text { Total } \\
\text { vehicles } \\
\text { per hour }\end{array}$} & \multicolumn{3}{|c|}{ Directional information } \\
\hline & & & $\begin{array}{c}\text { Turning } \\
\text { movement }\end{array}$ & Percent & Counts \\
\hline \multirow[t]{3}{*}{ U.S. 321 Intersection w/ Foothills Pkwy 8B } & FH Pkwy 8B & 220 & $\begin{array}{l}\text { Right Turn } \\
\text { Left Turn }\end{array}$ & $\begin{array}{r}100.00 \% \\
0.00 \%\end{array}$ & $\begin{array}{r}220 \\
0\end{array}$ \\
\hline & U.S. 321 WB & 929 & $\begin{array}{l}\text { Right Turn } \\
\text { Through }\end{array}$ & $\begin{array}{r}0.00 \% \\
100.00 \%\end{array}$ & $\begin{array}{r}0 \\
929\end{array}$ \\
\hline & U.S. $321 \mathrm{~EB}$ & 559 & $\begin{array}{l}\text { Through } \\
\text { Left Tum }\end{array}$ & $\begin{array}{l}72.81 \% \\
27.19 \%\end{array}$ & $\begin{array}{l}407 \\
152\end{array}$ \\
\hline
\end{tabular}

Table K.52. Weekend P.M. peak-hour intersection volume projections for 2006-interchange at U.S. 321 (Webb Creek Rd. connection)-8B opened prior to 8C completion (continued)

\begin{tabular}{|c|c|c|c|c|c|}
\hline \multirow[b]{2}{*}{ Intersection location } & \multirow[b]{2}{*}{ Approach } & \multirow{2}{*}{$\begin{array}{c}\text { Total } \\
\text { vehicles } \\
\text { per hour }\end{array}$} & \multicolumn{3}{|c|}{ Directional information } \\
\hline & & & $\begin{array}{c}\text { Turning } \\
\text { movement }\end{array}$ & Percent & Counts \\
\hline \multirow[t]{3}{*}{ Foothills Pkwy 8B/Webb Creek Rd to US 321} & FH Pkwy 8B & 270 & $\begin{array}{l}\text { Right Tum } \\
\text { Left Tum }\end{array}$ & $\begin{array}{r}90.74 \% \\
9.26 \%\end{array}$ & $\begin{array}{r}245 \\
25\end{array}$ \\
\hline & U.S. $321 \mathrm{WB}$ & 954 & $\begin{array}{l}\text { Right Turn } \\
\text { Through }\end{array}$ & $\begin{array}{r}2.62 \% \\
97.38 \%\end{array}$ & $\begin{array}{r}25 \\
929\end{array}$ \\
\hline & U.S. $321 \mathrm{~EB}$ & 584 & $\begin{array}{l}\text { Through } \\
\text { Left Turn }\end{array}$ & $\begin{array}{l}69.69 \% \\
30.31 \%\end{array}$ & $\begin{array}{l}407 \\
177\end{array}$ \\
\hline
\end{tabular}


Table K.53. Weekday A.M. peak-hour intersection volume projections for 2026-interchange at U.S. 321 (both options)-8B opened prior to completion of $8 \mathrm{C}$

\begin{tabular}{|c|c|c|c|c|c|}
\hline \multirow[b]{2}{*}{ Intersection location } & \multirow[b]{2}{*}{ Approach } & \multirow{2}{*}{$\begin{array}{c}\text { Total } \\
\text { vehicles } \\
\text { per hour }\end{array}$} & \multicolumn{3}{|c|}{ Directional information } \\
\hline & & & $\begin{array}{c}\text { Turning } \\
\text { movement }\end{array}$ & Percent & Counts \\
\hline \multirow[t]{12}{*}{ U.S. 321 Intersection w/ Foothills Pkwy } & FH Pkwy 8A WB & 300 & Right Turn & $12.00 \%$ & 36 \\
\hline & & & Through & $10.00 \%$ & 30 \\
\hline & & & Left Tum & $78.00 \%$ & 234 \\
\hline & FH Pkwy 8B EB & 152 & Right Turn & $5.92 \%$ & 9 \\
\hline & & & Through & $57.89 \%$ & 88 \\
\hline & & & Left Turn & $36.18 \%$ & 55 \\
\hline & U.S. $321 \mathrm{SB}$ & 680 & Right Tum & $11.32 \%$ & 77 \\
\hline & & & Through & $80.15 \%$ & 545 \\
\hline & & & Left Turn & $8.53 \%$ & 58 \\
\hline & U.S. $321 \mathrm{NB}$ & 849 & Right Tum & $20.49 \%$ & 174 \\
\hline & & & Through & $79.03 \%$ & 671 \\
\hline & & & Left Turn & $0.47 \%$ & 4 \\
\hline \multirow[t]{6}{*}{ U.S. 321 Convergence w/ SR 32} & U.S. $321 \mathrm{~EB}$ & 547 & Right Turn & $12.43 \%$ & 68 \\
\hline & & & Left Turn & $87.57 \%$ & 479 \\
\hline & U.S. $321 \mathrm{SB}$ & 306 & Right Tum & $73.53 \%$ & 225 \\
\hline & & & Through & $26.47 \%$ & 81 \\
\hline & SR $32 \mathrm{NB}$ & 90 & Through & $68.89 \%$ & 62 \\
\hline & & & Left Turn & $31.11 \%$ & 28 \\
\hline \multirow[t]{6}{*}{ U.S. 321 Intersection w/ SR 416} & SR $416 \mathrm{SB}$ & 176 & Right Turn & $81.25 \%$ & 143 \\
\hline & & & Left Turn & $18.75 \%$ & 33 \\
\hline & U.S. 321 WB & 1091 & Right Turn & $1.65 \%$ & 18 \\
\hline & & & Through & $98.35 \%$ & 1073 \\
\hline & U.S. $321 \mathrm{~EB}$ & 634 & Through & $93.06 \%$ & 590 \\
\hline & & & Left Tum & $6.94 \%$ & 44 \\
\hline \multirow[t]{6}{*}{ SR 416 Intersection w/ Webb Creek Rd } & Webb Cr Rd WB & 82 & Right Turn & $3.66 \%$ & 3 \\
\hline & & & Left Turn & $96.34 \%$ & 79 \\
\hline & SR 416 SB & 65 & Through & $96.92 \%$ & 63 \\
\hline & & & Left Turn & $3.08 \%$ & 2 \\
\hline & SR $416 \mathrm{NB}$ & 115 & Right Turn & $48.70 \%$ & 56 \\
\hline & & & Through & $51.30 \%$ & 59 \\
\hline \multirow[t]{12}{*}{ SR 416 Intersection w/ Foothills Pkwy 8B/8C } & FH Pkwy 8B WB & 0 & Right Turn & & 0 \\
\hline & & & Through & & 0 \\
\hline & & & Left Tum & & 0 \\
\hline & FH Pkwy 8C EB & 0 & Right Turn & & 0 \\
\hline & & & Through & & 0 \\
\hline & & & Left Turn & & 0 \\
\hline & SR 416 SB & 142 & Right Turn & $0.00 \%$ & 0 \\
\hline & & & Through & $100.00 \%$ & 142 \\
\hline & & & Left Turn & $0.00 \%$ & 0 \\
\hline & SR $416 \mathrm{NB}$ & 115 & Right Tum & $0.00 \%$ & 0 \\
\hline & & & Through & $100.00 \%$ & 115 \\
\hline & & & Left Turn & $0.00 \%$ & 0 \\
\hline
\end{tabular}


Table K.53. Weekday A.M. peak-hour intersection volume projections for 2026 -interchange at U.S. 321 (directly connected)-8B opened prior to $8 \mathrm{C}$ completion (continued)

\begin{tabular}{|c|c|c|c|c|c|}
\hline \multirow[b]{2}{*}{ Intersection location } & \multirow[b]{2}{*}{ Approach } & \multirow{2}{*}{$\begin{array}{c}\text { Total } \\
\text { vehicles } \\
\text { per hour }\end{array}$} & \multicolumn{3}{|c|}{ Directional information } \\
\hline & & & $\begin{array}{c}\text { Turning } \\
\text { movement }\end{array}$ & Percent. & Counts \\
\hline \multirow[t]{3}{*}{ U.S. 321 Intersection w/ Foothills Pkwy 8B } & FH Pkwy 8B & 102 & $\begin{array}{l}\text { Right Turn } \\
\text { Left Turn }\end{array}$ & $\begin{array}{r}100.00 \% \\
0.00 \%\end{array}$ & $\begin{array}{r}102 \\
0\end{array}$ \\
\hline & U.S. 321 WB & 976 & $\begin{array}{l}\text { Right Turn } \\
\text { Through }\end{array}$ & $\begin{array}{r}0.00 \% \\
100.00 \%\end{array}$ & $\begin{array}{r}0 \\
976\end{array}$ \\
\hline & U.S. $321 \mathrm{~EB}$ & 606 & $\begin{array}{l}\text { Through } \\
\text { Left Turn }\end{array}$ & $\begin{array}{l}74.92 \% \\
25.08 \%\end{array}$ & $\begin{array}{l}454 \\
152\end{array}$ \\
\hline
\end{tabular}

Table K.53. Weekday A.M. peak-hour intersection volume projections for 2026 -interchange at U.S. 321 (Webb Creek Rd. connection)-8B opened prior to 8C completion (continued)

\begin{tabular}{|c|c|c|c|c|c|}
\hline \multirow[b]{2}{*}{ Intersection location } & \multirow[b]{2}{*}{ Approach } & \multirow{2}{*}{$\begin{array}{c}\text { Total } \\
\text { vehicles } \\
\text { per hour }\end{array}$} & \multicolumn{3}{|c|}{ Directional information } \\
\hline & & & $\begin{array}{c}\text { Turning } \\
\text { movement }\end{array}$ & Percent & Counts \\
\hline \multirow[t]{3}{*}{ Foothills Pkwy 8B/Webb Creek Rd to US 321} & FH Pkwy 8B & 172 & $\begin{array}{l}\text { Right Turn } \\
\text { Left Turn }\end{array}$ & $\begin{array}{l}79.65 \% \\
20.35 \%\end{array}$ & $\begin{array}{r}137 \\
35\end{array}$ \\
\hline & U.S. $321 \mathrm{WB}$ & 1011 & $\begin{array}{l}\text { Right Turn } \\
\text { Through }\end{array}$ & $\begin{array}{r}3.46 \% \\
96.54 \%\end{array}$ & $\begin{array}{r}35 \\
976\end{array}$ \\
\hline & U.S. $321 \mathrm{~EB}$ & 641 & $\begin{array}{l}\text { Through } \\
\text { Left Tum }\end{array}$ & $\begin{array}{l}70.83 \% \\
29.17 \% \\
\end{array}$ & $\begin{array}{l}454 \\
187\end{array}$ \\
\hline
\end{tabular}


Table K.54. Weekday P.M. peak-hour intersection volume projections for 2026-interchange at U.S. 321 (both options)-8B opened prior to completion of $8 \mathrm{C}$

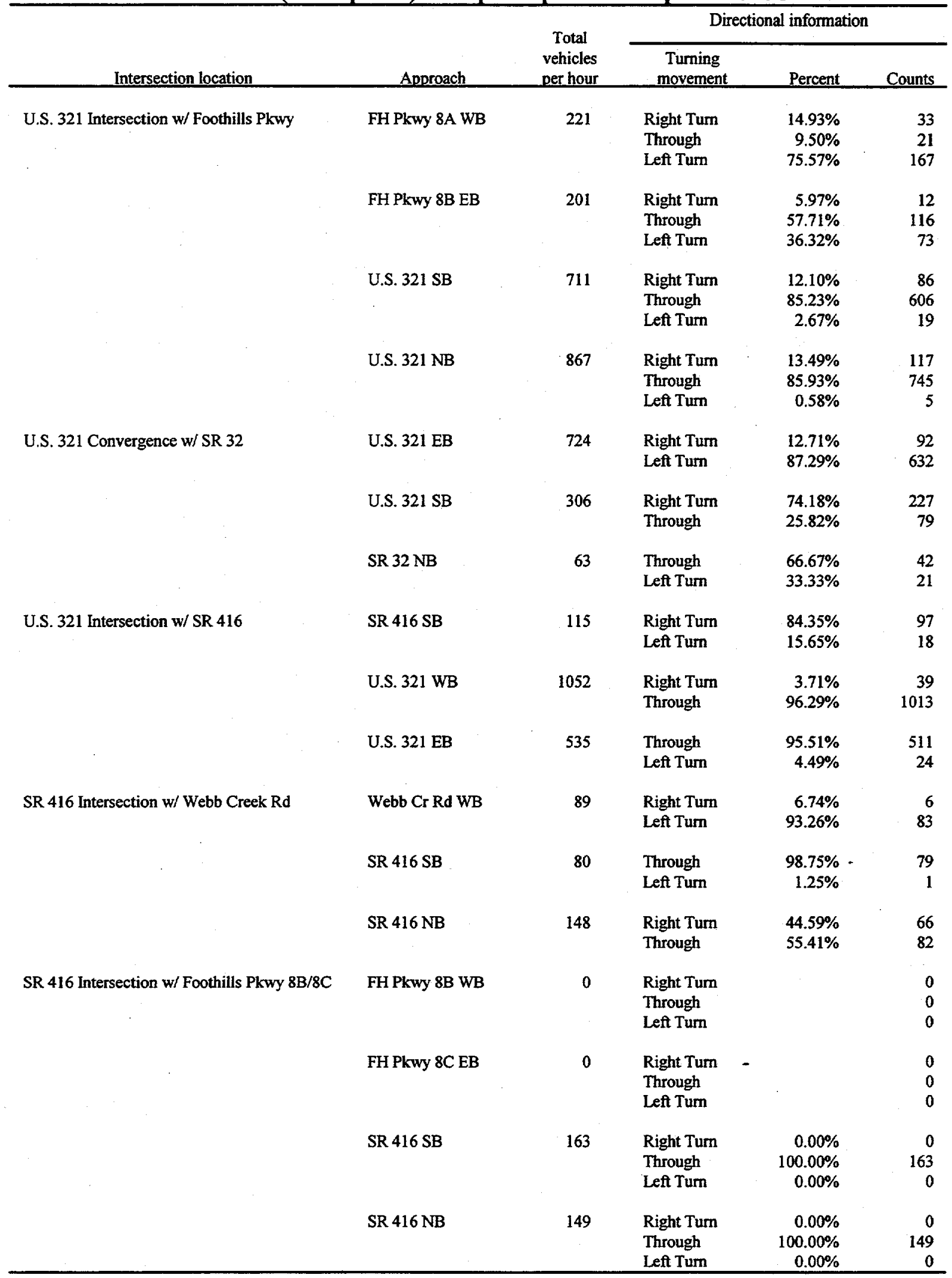


Table K.54. Weekday P.M. peak-hour intersection volume projections for 2026 -interchange at U.S. 321 (directly connected) $-8 B$ opened prior to $8 \mathrm{C}$ completion (continued)

\begin{tabular}{|c|c|c|c|c|c|}
\hline \multirow[b]{2}{*}{ Intersection location } & \multirow[b]{2}{*}{ Approach } & \multirow{2}{*}{$\begin{array}{c}\text { Total } \\
\text { vehicles } \\
\text { per hour }\end{array}$} & \multicolumn{3}{|c|}{ Directional information } \\
\hline & & & $\begin{array}{c}\text { Turning } \\
\text { movement }\end{array}$ & Percent & Counts \\
\hline \multirow[t]{3}{*}{ U.S. 321 Intersection w/ Foothills Pkwy 8B } & FH Pkwy 8B & 135 & $\begin{array}{l}\text { Right Turn } \\
\text { Left Turn }\end{array}$ & $\begin{array}{r}100.00 \% \\
0.00 \%\end{array}$ & $\begin{array}{r}135 \\
0\end{array}$ \\
\hline & U.S. 321 WB & 922 & $\begin{array}{l}\text { Right Turn } \\
\text { Through }\end{array}$ & $\begin{array}{r}0.00 \% \\
100.00 \%\end{array}$ & $\begin{array}{r}0 \\
922\end{array}$ \\
\hline & U.S. 321 EB & 593 & $\begin{array}{l}\text { Through } \\
\text { Left Turn }\end{array}$ & $\begin{array}{l}66.27 \% \\
33.73 \%\end{array}$ & $\begin{array}{l}393 \\
200\end{array}$ \\
\hline
\end{tabular}

Table K.54. Weekday P.M. peak-hour intersection volume projections for 2026-interchange at U.S. 321 (Webb Creek Rd. connection)-8B opened prior to 8C completion (continued)

\begin{tabular}{|c|c|c|c|c|c|}
\hline \multirow[b]{2}{*}{ Intersection location } & \multirow[b]{2}{*}{ Approach } & \multirow{2}{*}{$\begin{array}{c}\text { Total } \\
\text { vehicies } \\
\text { per hour }\end{array}$} & \multicolumn{3}{|c|}{ Directional information } \\
\hline & & & $\begin{array}{c}\text { Tuming } \\
\text { movement }\end{array}$ & Percent & Counts \\
\hline \multirow[t]{3}{*}{ Foothills Pkwy 8B/Webb Creek Rd to US 321} & FH Pkwy 8B & 215 & $\begin{array}{l}\text { Right Tum } \\
\text { Left Turn }\end{array}$ & $\begin{array}{l}81.40 \% \\
18.60 \%\end{array}$ & $\begin{array}{r}175 \\
40\end{array}$ \\
\hline & U.S. 321 WB & 962 & $\begin{array}{l}\text { Right Tum } \\
\text { Through }\end{array}$ & $\begin{array}{r}4.16 \% \\
95.84 \%\end{array}$ & $\begin{array}{r}40 \\
922\end{array}$ \\
\hline & U.S. 321 EB & 633 & $\begin{array}{l}\text { Through } \\
\text { Left Turn }\end{array}$ & $\begin{array}{l}62.09 \% \\
37.91 \% \\
\end{array}$ & $\begin{array}{l}393 \\
240 \\
\end{array}$ \\
\hline
\end{tabular}


Table K.55. Weekend A.M. peak-hour intersection volume projections for 2026-interchange at U.S. 321 (both options)-8B opened prior to completion of 8C

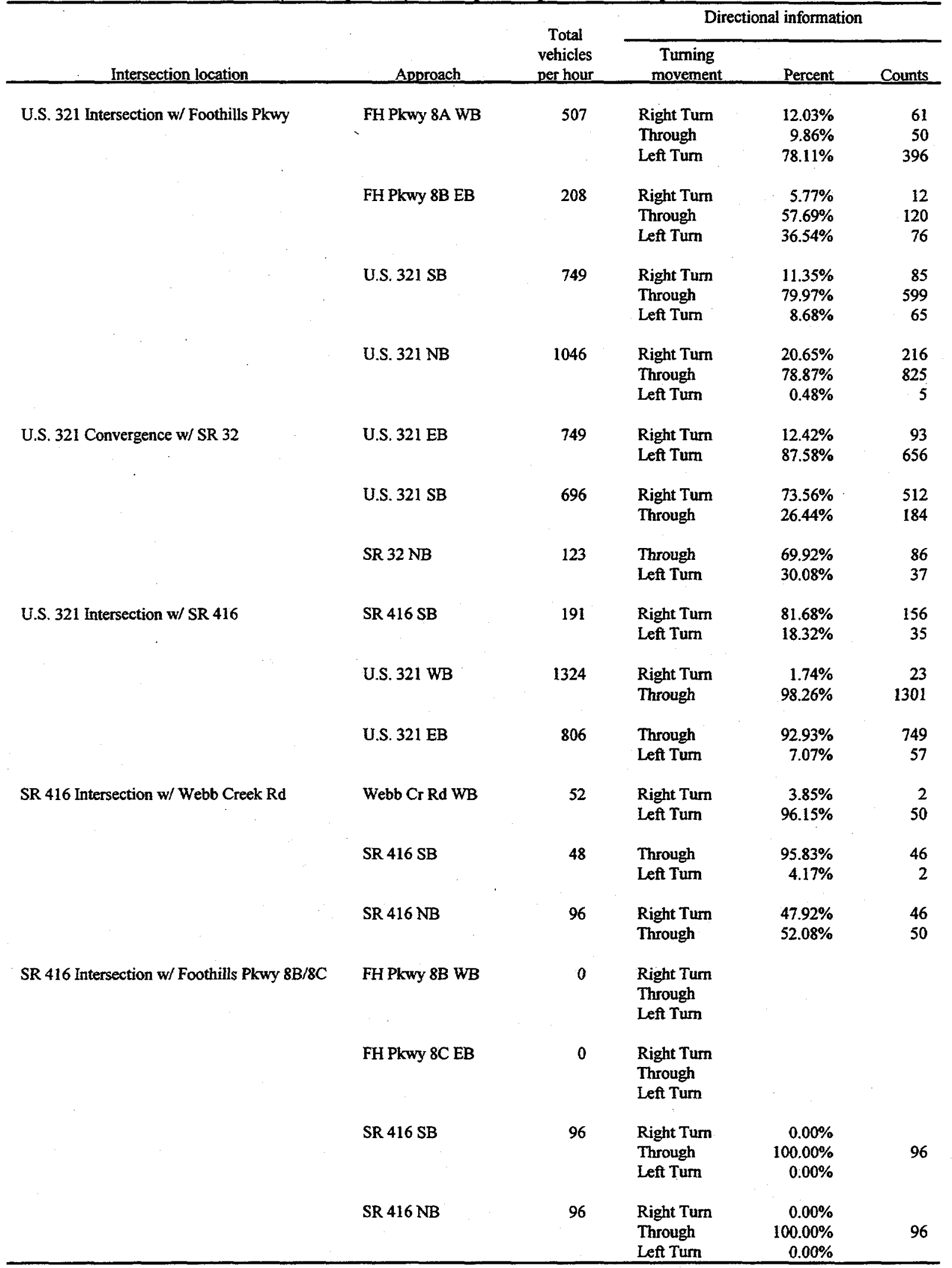


Table K.55. Weekend A.M. peak-hour intersection volume projections for 2026 -interchange at U.S. 321 (directly connected) $-8 \mathrm{~B}$ opened prior to $8 \mathrm{C}$ completion (continued)

\begin{tabular}{|c|c|c|c|c|c|}
\hline \multirow[b]{2}{*}{ Intersection location } & \multirow[b]{2}{*}{ Approach } & \multirow{2}{*}{$\begin{array}{c}\text { Total } \\
\text { vehicles } \\
\text { per hour }\end{array}$} & \multicolumn{3}{|c|}{ Directional information } \\
\hline & & & $\begin{array}{c}\text { Turning } \\
\text { movement }\end{array}$ & Percent & Counts \\
\hline \multirow[t]{3}{*}{ U.S. 321 Intersection w/ Foothills Pkwy 8B } & FH Pkwy 8B & 140 & $\begin{array}{l}\text { Right Tum } \\
\text { Left Tum }\end{array}$ & $\begin{array}{r}100.00 \% \\
0.00 \%\end{array}$ & $\begin{array}{r}140 \\
0\end{array}$ \\
\hline & U.S. 321 WB & 1184 & $\begin{array}{l}\text { Right Turn } \\
\text { Through }\end{array}$ & $\begin{array}{r}0.00 \% \\
100.00 \%\end{array}$ & $\begin{array}{r}0 \\
1184\end{array}$ \\
\hline & U.S. $321 \mathrm{~EB}$ & 784 & $\begin{array}{l}\text { Through } \\
\text { Left Tum }\end{array}$ & $\begin{array}{l}73.47 \% \\
26.53 \%\end{array}$ & $\begin{array}{l}576 \\
208\end{array}$ \\
\hline
\end{tabular}

Table K.55. Weekend A.M. peak-hour intersection volume projections for 2026-interchange at U.S. 321 (Webb Creek Rd. connection)-8B opened prior to 8C completion (continued)

\begin{tabular}{|c|c|c|c|c|c|}
\hline \multirow[b]{2}{*}{ Intersection location } & \multirow[b]{2}{*}{ Approach } & \multirow{2}{*}{$\begin{array}{c}\text { Total } \\
\text { vehicles } \\
\text { per hour }\end{array}$} & \multicolumn{3}{|c|}{ Directional information } \\
\hline & & & $\begin{array}{c}\text { Tuming } \\
\text { movement }\end{array}$ & Percent & Counts \\
\hline \multirow[t]{3}{*}{ Foothills Pkwy 8B/Webb Creek Rd to US 321} & FH Pkwy 8B & 190 & $\begin{array}{l}\text { Right Turn } \\
\text { Left Turn }\end{array}$ & $\begin{array}{l}86.84 \% \\
13.16 \%\end{array}$ & $\begin{array}{r}165 \\
25\end{array}$ \\
\hline & U.S. 321 WB & 1209 & $\begin{array}{l}\text { Right Tum } \\
\text { Through }\end{array}$ & $\begin{array}{r}2.07 \% \\
97.93 \%\end{array}$ & $\begin{array}{r}25 \\
1184\end{array}$ \\
\hline & U.S. $321 \mathrm{~EB}$ & 809 & $\begin{array}{l}\text { Through } \\
\text { Left Tum }\end{array}$ & $\begin{array}{l}71.20 \% \\
28.80 \%\end{array}$ & $\begin{array}{l}576 \\
233\end{array}$ \\
\hline
\end{tabular}


Table K.56. Weekend P.M. peak-hour intersection volume projections for 2026 -interchange at U.S. 321 (both options)-8B opened prior to completion of $8 \mathrm{C}$

\begin{tabular}{|c|c|c|c|c|c|}
\hline \multirow[b]{2}{*}{ Intersection location } & \multirow[b]{2}{*}{ Approach } & \multirow{2}{*}{$\begin{array}{c}\text { Total } \\
\text { vehicles } \\
\text { per hour }\end{array}$} & \multicolumn{3}{|c|}{ Directional information } \\
\hline & & & $\begin{array}{c}\text { Turning } \\
\text { movement }\end{array}$ & Percent & Counts \\
\hline \multirow[t]{12}{*}{ U.S. 321 Intersection w/ Foothills Pkwy } & FH Pkwy 8A WB & 308 & Right Turn & $15.26 \%$ & 47 \\
\hline & & & Through & $9.42 \%$ & 29 \\
\hline & & & Left Turn & $75.32 \%$ & 232 \\
\hline & FH Pkwy 8B EB & 241 & Right Turn & $5.81 \%$ & 14 \\
\hline & & & Through & $57.68 \%$ & 139 \\
\hline & & & Left Turn & $36.51 \%$ & 88 \\
\hline & U.S. $321 \mathrm{SB}$ & 833 & Right Turn & $12.12 \%$ & 101 \\
\hline & & & Through & $85.23 \%$ & 710 \\
\hline & & & Left Turn & $2.64 \%$ & 22 \\
\hline & U.S. $321 \mathrm{NB}$ & 991 & Right Tum & $13.42 \%$ & 133 \\
\hline & & & Through & $86.07 \%$ & 853 \\
\hline & & & Left Turn & $0.50 \%$ & 5 \\
\hline \multirow[t]{6}{*}{ U.S. 321 Convergence w/ SR 32} & U.S. $321 \mathrm{~EB}$ & 871 & Right Turn & $12.74 \%$ & 111 \\
\hline & & & Left Turn & $87.26 \%$ & 760 \\
\hline & U.S. $321 \mathrm{SB}$ & 580 & Right Turn & $73.97 \%$ & 429 \\
\hline & & & Through & $26.03 \%$ & 151 \\
\hline & SR $32 \mathrm{NB}$ & 82 & Through & $65.85 \%$ & 54 \\
\hline & & & Left Tum & $34.15 \%$ & 28 \\
\hline \multirow[t]{6}{*}{ U.S. 321 Intersection w/ SR 416} & SR $416 \mathrm{SB}$ & 224 & Right Turn & $83.48 \%$ & 187 \\
\hline & & & Left Turn & $16.52 \%$ & 37 \\
\hline & U.S. 321 WB & 1408 & Right Turn & $3.76 \%$ & 53 \\
\hline & & & Through & $96.24 \%$ & 1355 \\
\hline & U.S. $321 \mathrm{~EB}$ & 762 & Through & $95.54 \%$ & 728 \\
\hline & & & Left Turn & $4.46 \%$ & 34 \\
\hline \multirow[t]{6}{*}{ SR 416 Intersection w/ Webb Creek Rd } & Webb Cr Rd WB & 68 & Right Turn & $7.35 \%$ & 5 \\
\hline & & & Left Tum & $92.65 \%$ & 63 \\
\hline & SR 416 SB & 110 & Through & $99.09 \%$ & 109 \\
\hline & & & Left Turn & $0.91 \%$ & 1 \\
\hline & SR 416 NB & 148 & Right Turn & $44.59 \%$ & 66 \\
\hline & & & Through & $55.41 \%$ & 82 \\
\hline \multirow[t]{12}{*}{ SR 416 Intersection w/ Foothills Pkwy 8B/8C } & FH Pkwy 8B WB & 0 & Right Turn & & 0 \\
\hline & & & Through & & 0 \\
\hline & & & Left Tum & & 0 \\
\hline & FH Pkwy 8C EB & 0 & Right Turn & & 0 \\
\hline & & & Through & & 0 \\
\hline & & & Left Turn & & 0 \\
\hline & SR 416 SB & 171 & Right Tum & $0.00 \%$ & 0 \\
\hline & & & Through & $100.00 \%$ & 171 \\
\hline & & & Left Turn & $0.00 \%$ & 0 \\
\hline & SR $416 \mathrm{NB}$ & 149 & Right Turn & $0.00 \%$ & 0 \\
\hline & & & Through & $100.00 \%$ & 149 \\
\hline & & & Left Turn & $0.00 \%$ & 0 \\
\hline
\end{tabular}


Table K.56. Weekend.P.M. peak-hour intersection volume projections for 2026-interchange at U.S. 321 (directly connected)-8B opened prior to $8 \mathrm{C}$ completion (continued)

\begin{tabular}{|c|c|c|c|c|c|}
\hline \multirow[b]{2}{*}{ Intersection location } & \multirow[b]{2}{*}{ Approach } & \multirow{2}{*}{$\begin{array}{c}\text { Total } \\
\text { vehicles } \\
\text { per hour }\end{array}$} & \multicolumn{3}{|c|}{ Directional information } \\
\hline & & & $\begin{array}{c}\text { Turning } \\
\text { movement }\end{array}$ & Percent & Counts \\
\hline \multirow[t]{3}{*}{ U.S. 321 Intersection w/ Foothills Pkwy 8B } & FH Pkwy 8B & 162 & $\begin{array}{l}\text { Right Turn } \\
\text { Left Turn }\end{array}$ & $\begin{array}{r}100.00 \% \\
0.00 \%\end{array}$ & $\begin{array}{r}162 \\
0\end{array}$ \\
\hline & U.S. 321 WB & 1233 & $\begin{array}{l}\text { Right Turn } \\
\text { Through }\end{array}$ & $\begin{array}{r}0.00 \% \\
100.00 \%\end{array}$ & $\begin{array}{r}0 \\
1233\end{array}$ \\
\hline & U.S. $321 \mathrm{~EB}$ & 801 & $\begin{array}{l}\text { Through } \\
\text { Left Turn }\end{array}$ & $\begin{array}{l}69.91 \% \\
30.09 \%\end{array}$ & $\begin{array}{l}560 \\
241\end{array}$ \\
\hline
\end{tabular}

Table K.56. Weekend P.M. peak-hour intersection volume projections for 2026-interchange at U.S. 321 (Webb Creek Rd. connection)-8B opened prior to 8C completion (continued)

\begin{tabular}{|c|c|c|c|c|c|}
\hline \multirow[b]{2}{*}{ Intersection location } & \multirow[b]{2}{*}{ Approach } & \multirow{2}{*}{$\begin{array}{c}\text { Total } \\
\text { vehicles } \\
\text { per hour }\end{array}$} & \multicolumn{3}{|c|}{ Directional information } \\
\hline & & & $\begin{array}{c}\text { Turning } \\
\text { movement }\end{array}$ & Percent & Counts \\
\hline \multirow[t]{3}{*}{ Foothills Pkwy 8B/Webb Creek Rd to US 321} & FH Pkwy 8B & 232 & $\begin{array}{l}\text { Right Turn } \\
\text { Left Tum }\end{array}$ & $\begin{array}{l}84.91 \% \\
15.09 \%\end{array}$ & $\begin{array}{r}197 \\
35\end{array}$ \\
\hline & U.S. 321 WB & 1268 & $\begin{array}{l}\text { Right Turn } \\
\text { Through }\end{array}$ & $\begin{array}{r}2.76 \% \\
97.24 \%\end{array}$ & $\begin{array}{r}35 \\
1233\end{array}$ \\
\hline & U.S. $321 \mathrm{~EB}$ & 836 & $\begin{array}{l}\text { Through } \\
\text { Left Turn }\end{array}$ & $\begin{array}{l}66.99 \% \\
33.01 \%\end{array}$ & $\begin{array}{l}560 \\
276\end{array}$ \\
\hline
\end{tabular}


Table K.57. Weekday A.M. peak-hour intersection volume projections for $2006-$ no build alternative

\begin{tabular}{|c|c|c|c|c|c|}
\hline \multirow[b]{2}{*}{ Intersection location } & \multirow[b]{2}{*}{ Approach } & \multirow{2}{*}{$\begin{array}{c}\text { Total } \\
\text { vehicles } \\
\text { per hour }\end{array}$} & \multicolumn{3}{|c|}{ Directional information } \\
\hline & & & $\begin{array}{c}\text { Turning } \\
\text { movement }\end{array}$ & Percent & Counts \\
\hline \multirow[t]{12}{*}{ U.S. 321 Intersection w/ Foothills Pkwy } & FH Pkwy 8A WB. & 207 & Right Turn & $14.01 \%$ & 29 \\
\hline & & & Through & $0.00 \%$ & 0 \\
\hline & & & Left Turn & $85.99 \%$ & 178 \\
\hline & FH Pkwy 8B EB & 0 & Right Turn & & 0 \\
\hline & & & Through & & 0 \\
\hline & & & Left Turn & & 0 \\
\hline & U.S. $321 \mathrm{SB}$ & 536 & Right Turn & $0.00 \%$ & 0 \\
\hline & & & Through & $90.11 \%$ & 483 \\
\hline & & & Left Turn & $9.89 \%$ & 53 \\
\hline & U.S. $321 \mathrm{NB}$ & 705 & Right Tum & $20.71 \%$ & 146 \\
\hline & & & Through & $79.29 \%$ & 559 \\
\hline & & & Left Turn & $0.00 \%$ & 0 \\
\hline \multirow[t]{6}{*}{ U.S. 321 Convergence w/ SR 32} & U.S. $321 \mathrm{~EB}$ & 466 & Right Tum & $12.66 \%$ & 59 \\
\hline & & & Left Tum & $87.34 \%$ & 407 \\
\hline & U.S. $321 \mathrm{SB}$ & 259 & Right Turn & $75.68 \%$ & 196 \\
\hline & & & Through & $24.32 \%$ & 63 \\
\hline & SR $32 \mathrm{NB}$ & 77 & Through & $66.23 \%$ & 51 \\
\hline & & & Left Turn & $33.77 \%$ & 26 \\
\hline \multirow[t]{6}{*}{ U.S. 321 Intersection w/ SR 416} & SR 416 SB & 125 & Right Turn & $76.00 \%$ & 95 \\
\hline & & & Left Tum & $24.00 \%$ & 30 \\
\hline & U.S. 321 WB & 803 & Right Turn & $4.23 \%$ & 34 \\
\hline & & & Through & $95.77 \%$ & 769 \\
\hline & U.S. $321 \mathrm{~EB}$ & 470 & Through & $.76 .38 \%$ & 359 \\
\hline & & & Left Turn & $23.62 \%$ & 111 \\
\hline \multirow[t]{6}{*}{ SR 416 Intersection w/ Webb Creek Rd } & Webb CrRd WB & 54 & Right Turn & $3.70 \%$ & 2 \\
\hline & & & Left Turn & $96.30 \%$ & 52 \\
\hline & SR $416 \mathrm{SB}$ & 42 & Through & $97.62 \%$ & 41 \\
\hline & & & Left Turn & $2.38 \%$ & 1 \\
\hline & SR $416 \mathrm{NB}$ & 66 & Right Turn & $50.00 \%$ & 33 \\
\hline & & & Through & $50.00 \%$ & 33 \\
\hline \multirow[t]{12}{*}{ SR 416 Intersection w/Foothills Pkwy $8 \mathrm{~B} / 8 \mathrm{C}$} & FH Pkwy 8B wB & 0 & Right Turn & & 0 \\
\hline & & & Through & & 0 \\
\hline & & . & Left Turn & & 0 \\
\hline & FH Pkwy 8C EB & 0 & Right Turn & & 0 \\
\hline & & & Through & & 0 \\
\hline & & & Left Turn & & 0 \\
\hline & SR 416 SB & 93 & Right Tum & $0.00 \%$ & 0 \\
\hline & & & Through & $100.00 \%$ & 93 \\
\hline & & & Left Tum & $0.00 \%$ & 0 \\
\hline & SR 416 NB & 66 & Right Turn & $0.00 \%$ & 0 \\
\hline & & & Through & $100.00 \%$ & 66 \\
\hline & & & Left Tum & $0.00 \%$ & 0 \\
\hline
\end{tabular}


Table K.58. Weekday P.M. peak-hour intersection volume projections for 2006-no build alternative

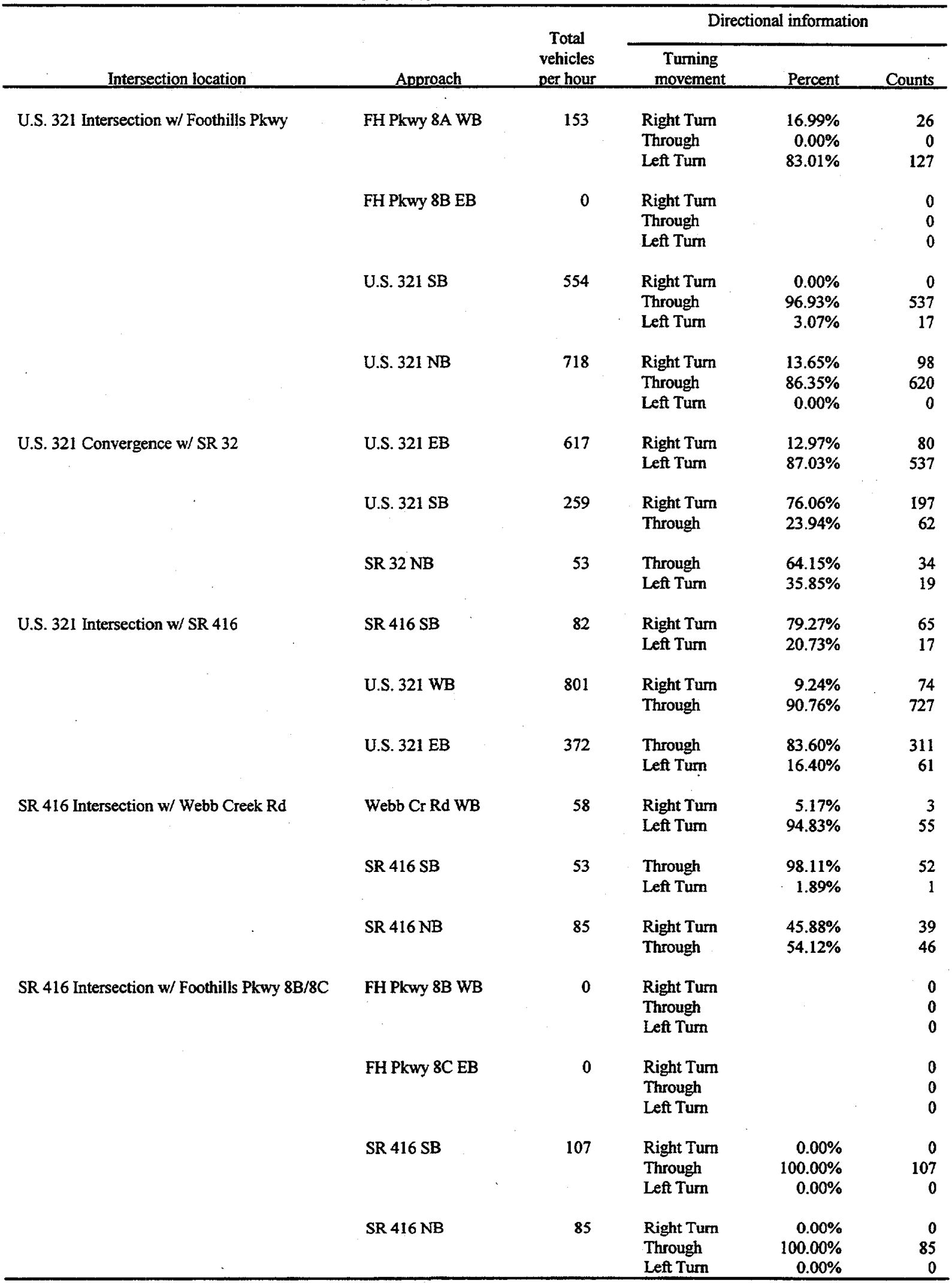


Table K.59. Weekend A.M. peak-hour intersection volume projections for 2006-no build alternative

\begin{tabular}{|c|c|c|c|c|c|}
\hline \multirow[b]{2}{*}{ Intersection location } & \multirow[b]{2}{*}{ Approach } & \multirow{2}{*}{$\begin{array}{c}\text { Total } \\
\text { vehicles } \\
\text { per hour }\end{array}$} & \multicolumn{3}{|c|}{ Directional information } \\
\hline & & & $\begin{array}{l}\text { Turning } \\
\text { movement }\end{array}$ & Percent & Counts \\
\hline \multirow[t]{12}{*}{ U.S. 321 Intersection w/ Foothilis Pkwy } & FH Pkwy 8A WB & 350 & Right Tum & $14.00 \%$ & 49 \\
\hline & & & Through & $0.00 \%$ & 0 \\
\hline & & & Left Turn & $.86 .00 \%$ & \\
\hline & FH Pkwy 8B EB & 0 & Right Turn & $0.00 \%$ & 0 \\
\hline & & & Through & $0.00 \%$ & 0 \\
\hline & & & Left Turn & $0.00 \%$ & 0 \\
\hline & U.S. $321 \mathrm{SB}$ & 590 & Right Tum & $0.00 \%$ & 0 \\
\hline & & & Through & $90.00 \%$ & 531 \\
\hline & & & Left Turn & $10.00 \%$ & 59 \\
\hline & U.S. $321 \mathrm{NB}$ & 868 & Right Tum & $20.85 \%$ & 181 \\
\hline & & & Through & $79.15 \%$ & 687 \\
\hline & & & Left Tum & $0.00 \%$ & 0 \\
\hline \multirow[t]{6}{*}{ U.S. 321 Convergence $w / S R 32$} & U.S. $321 \mathrm{~EB}$ & 638 & Right Tum & $12.70 \%$ & 81 \\
\hline & & & Left Turn & $87.30 \%$ & 557 \\
\hline & U.S. 321 SB & 589 & Right Tum & $75.72 \%$ & 446 \\
\hline & & & Through & $24.28 \%$ & 143 \\
\hline & SR 32 NB & 104 & Through & $67.31 \%$ & 70 \\
\hline & & & Left Turn & $32.69 \%$ & 34 \\
\hline \multirow[t]{6}{*}{ U.S. 321 Intersection w/ SR 416} & SR $416 \mathrm{SB}$ & 136 & Right Turn & $76.47 \%$ & 104 \\
\hline & & & Left Turn & $23.53 \%$ & 32 \\
\hline & U.S. 321 WB & 976 & Right Turn & $4.41 \%$ & 43 \\
\hline & & & Through & $95.59 \%$ & 933 \\
\hline & U.S. $321 \mathrm{~EB}$ & 598 & Through & $76.09 \%$ & 455 \\
\hline & & & Left Turn & $23.91 \%$ & 143 \\
\hline \multirow[t]{6}{*}{ SR 416 Intersection w/ Webb Creek Rd } & Webb Cr Rd WB & 34 & Right Turn & $2.94 \%$ & 1 \\
\hline & & & Left Turn & $97.06 \%$ & 33 \\
\hline & SR 416 SB & 31 & Through & $96.77 \%$ & 30 \\
\hline & & & Left Turn & $3.23 \%$ & 1 \\
\hline & SR $416 \mathrm{NB}$ & 55 & Right Turn & $49.09 \%$ & 27 \\
\hline & & & Through & $50.91 \%$ & 28 \\
\hline \multirow[t]{12}{*}{ SR 416 Intersection w/ Foothills Pkwy 8B/8C } & FH Pkwy 8B WB & 0 & Right Turn & $0.00 \%$ & 0 \\
\hline & & & Through & $0.00 \%$ & 0 \\
\hline & & & Left Turn & $0.00 \%$ & 0 \\
\hline & FH Pkwy 8C EB & 0 & Right Turn & $0.00 \%$ & 0 \\
\hline & & & Through & $0.00 \%$ & 0 \\
\hline & & & Left Tum & $0.00 \%$ & 0 \\
\hline & SR 416 SB & 63 & Right Tum & $0.00 \%$ & 0 \\
\hline & & & Through & $100.00 \%$ & 63 \\
\hline & & & Left Turn & $0.00 \%$ & 0 \\
\hline & SR 416 NB & 55 & Right Tum & $0.00 \%$ & 0 \\
\hline & & & Through & $100.00 \%$ & 55 \\
\hline & & & Left Turn & $0.00 \%$ & 0 \\
\hline
\end{tabular}


Table K.60. Weekend P.M. peak-hour intersection volume projections for 2006-no build alternative

\begin{tabular}{|c|c|c|c|c|c|}
\hline \multirow[b]{2}{*}{ Intersection location } & \multirow[b]{2}{*}{ Approach } & \multirow{2}{*}{$\begin{array}{c}\text { Total } \\
\text { vehicles } \\
\text { per hour }\end{array}$} & \multicolumn{3}{|c|}{ Directional information } \\
\hline & & & $\begin{array}{c}\text { Tuming } \\
\text { movement }\end{array}$ & Percent & Counts \\
\hline \multirow[t]{12}{*}{ U.S. 321 Intersection w/ Foothills Pkwy } & FH Pkwy 8A WB & 215 & Right Tum & $17.67 \%$ & 38 \\
\hline & & & Through & $0.00 \%$ & 0 \\
\hline & & & Left Tum & $82.33 \%$ & 177 \\
\hline & FH Pkwy 8B EB & 0 & Right Turn & & 0 \\
\hline & & & Through & & 0 \\
\hline & & & Left Turn & & 0 \\
\hline & U.S. $321 \mathrm{SB}$ & 650 & Right Turn & $0.00 \%$ & 0 \\
\hline & & & Through & $96.92 \%$ & 630 \\
\hline & & & Left Turn & $3.08 \%$ & 20 \\
\hline & U.S. $321 \mathrm{NB}$ & 821 & Right Turn & $13.52 \%$ & 111 \\
\hline & & & Through & $86.48 \%$ & 710 \\
\hline & & & Left Turn & $0.00 \%$ & 0 \\
\hline \multirow[t]{4}{*}{ U.S. 321 Convergence w/ SR 32} & U.S. $321 \mathrm{~EB}$ & 743 & Right Tum & $13.06 \%$ & 97 \\
\hline & & & Left Tum & $86.94 \%$ & 646 \\
\hline & U.S. $321 \mathrm{SB}$ & 490 & Right Turn & $76.12 \%$ & 373 \\
\hline & & & Through & $23.88 \%$ & 117 \\
\hline . & SR 32 NB & 70 & Through & $62.86 \%$ & 44 \\
\hline & & & Left Turn & $37.14 \%$ & 26 \\
\hline \multirow[t]{6}{*}{ U.S. 321 Intersection w/ SR 416} & SR $416 \mathrm{SB}$ & 158 & Right Tum & $78.48 \%$ & 124 \\
\hline & & & Left Turn & $21.52 \%$ & 34 \\
\hline & U.S. 321 WB & 1069 & Right Tum & $9.17 \%$ & 98 \\
\hline & & & Through & $90.83 \%$ & 971 \\
\hline & U.S. $321 \mathrm{~EB}$ & 527 & Through & $83.87 \%$ & 442 \\
\hline & & & Left Turn & $16.13 \%$ & 85 \\
\hline \multirow[t]{6}{*}{ SR 416 Intersection w/ Webb Creek Rd } & Webb Cr Rd WB & 43 & Right Turn & $4.65 \%$ & 2 \\
\hline & & & Left Turn & $95.35 \%$ & 41 \\
\hline & SR 416 SB & 72 & Through & $98.61 \%$ & 71 \\
\hline & & & Left Tum & $1.39 \%$ & 1 \\
\hline & SR $416 \mathrm{NB}$ & 85 & Right Turn & $45.88 \%$ & 39 \\
\hline & & & Through & $54.12 \%$ & 46 \\
\hline \multirow[t]{12}{*}{ SR 416 Intersection w/ Foothills Pkwy 8B/8C } & FH Pkwy 8B WB & 0 & Right Tum & & 0 \\
\hline & & & Through & & 0 \\
\hline & & & Left Turn & & 0 \\
\hline & FH Pkwy 8C EB & 0 & Right Turn & & 0 \\
\hline & & & Through & & 0 \\
\hline & & & Left Turn & & 0 \\
\hline & SR $416 \mathrm{SB}$ & 112 & Right Turn & $0.00 \%$ & 0 \\
\hline & & & Through & $100.00 \%$ & 112 \\
\hline & & & Left Turn & $0.00 \%$ & 0 \\
\hline & SR $416 \mathrm{NB}$ & 85 & Right Turn & $0.00 \%$ & 0 \\
\hline & & & Through & $100.00 \%$ & 85 \\
\hline & & & Left Turn & $0.00 \%$ & 0 \\
\hline
\end{tabular}


Table K.61. Weekday A.M. peak-hour intersection volume projections for 2026-no build alternative

\begin{tabular}{|c|c|c|c|c|c|}
\hline \multirow[b]{2}{*}{ Intersection location } & \multirow[b]{2}{*}{ Approach } & \multirow{2}{*}{$\begin{array}{c}\text { Total } \\
\text { vehicles } \\
\text { per hour }\end{array}$} & \multicolumn{3}{|c|}{ Directional information } \\
\hline & & & $\begin{array}{c}\text { Tuming } \\
\text { movement }\end{array}$ & Percent & Counts \\
\hline \multirow[t]{4}{*}{ U.S. 321 Intersection w/ Foothills Pkwy } & FH Pkwy 8A WB & 274 & $\begin{array}{l}\text { Right Tum } \\
\text { Through } \\
\text { Left Turn }\end{array}$ & $\begin{array}{r}13.14 \% \\
0.00 \% \\
86.86 \%\end{array}$ & $\begin{array}{r}36 \\
0 \\
238\end{array}$ \\
\hline & FH Pkwy 8B EB & 0 & $\begin{array}{l}\text { Right Turn } \\
\text { Through } \\
\text { Left Turn }\end{array}$ & & $\begin{array}{l}0 \\
0 \\
0\end{array}$ \\
\hline & U.S. $321 \mathrm{SB}$ & 680 & $\begin{array}{l}\text { Right Tum } \\
\text { Through } \\
\text { Left Turn }\end{array}$ & $\begin{array}{r}0.00 \% \\
91.47 \% \\
8.53 \%\end{array}$ & $\begin{array}{r}0 \\
622 \\
58\end{array}$ \\
\hline & U.S. $321 \mathrm{NB}$ & 915 & $\begin{array}{l}\text { Right Tum } \\
\text { Through } \\
\text { Left Turn }\end{array}$ & $\begin{array}{r}19.89 \% \\
80.11 \% \\
0.00 \%\end{array}$ & $\begin{array}{r}182 \\
733 \\
0\end{array}$ \\
\hline \multirow[t]{3}{*}{ U.S. 321 Convergence w/ SR 32} & U.S. $321 \mathrm{~EB}$ & 617 & $\begin{array}{l}\text { Right Turn } \\
\text { Left Tum }\end{array}$ & $\begin{array}{l}12.32 \% \\
87.68 \%\end{array}$ & $\begin{array}{r}76 \\
541\end{array}$ \\
\hline & U.S. $321 \mathrm{SB}$ & 340 & $\begin{array}{l}\text { Right Tum } \\
\text { Through }\end{array}$ & $\begin{array}{l}77.94 \% \\
22.06 \%\end{array}$ & $\begin{array}{r}265 \\
75\end{array}$ \\
\hline & SR 32 NB & 91 & $\begin{array}{l}\text { Through } \\
\text { Left Turn }\end{array}$ & $\begin{array}{l}64.84 \% \\
35.16 \%\end{array}$ & $\begin{array}{l}59 \\
32\end{array}$ \\
\hline \multirow[t]{3}{*}{ U.S. 321 Intersection w/ SR 416} & SR $416 \mathrm{SB}$ & 176 & $\begin{array}{l}\text { Right Turn } \\
\text { Left Tum }\end{array}$ & $\begin{array}{l}81.25 \% \\
18.75 \%\end{array}$ & $\begin{array}{r}143 \\
33\end{array}$ \\
\hline & U.S. $321 \mathrm{WB}$ & 1054 & $\begin{array}{l}\text { Right Turn } \\
\text { Through }\end{array}$ & $\begin{array}{r}1.71 \% \\
98.29 \%\end{array}$ & $\begin{array}{r}18 \\
1036\end{array}$ \\
\hline & U.S. 321 EB & 547 & $\begin{array}{l}\text { Through } \\
\text { Left Turn }\end{array}$ & $\begin{array}{r}91.96 \% \\
8.04 \%\end{array}$ & $\begin{array}{r}503 \\
44\end{array}$ \\
\hline \multirow[t]{3}{*}{ SR 416 Intersection w/ Webb Creek Rd } & Webb Cr Rd WB & 82 & $\begin{array}{l}\text { Right Turn } \\
\text { Left Turn }\end{array}$ & $\begin{array}{r}3.66 \% \\
96.34 \%\end{array}$ & $\begin{array}{r}3 \\
79\end{array}$ \\
\hline & SR 416 SB & 65 & $\begin{array}{l}\text { Through } \\
\text { Left Turn }\end{array}$ & $\begin{array}{r}96.92 \% \\
3.08 \%\end{array}$ & $\begin{array}{r}63 \\
2\end{array}$ \\
\hline & SR $416 \mathrm{NB}$ & 115 & $\begin{array}{l}\text { Right Tum } \\
\text { Through }\end{array}$ & $\begin{array}{l}48.70 \% \\
51.30 \%\end{array}$ & $\begin{array}{l}56 \\
59\end{array}$ \\
\hline \multirow[t]{4}{*}{ SR 416 Intersection w/ Foothills Pkwy 8B/8C } & FH Pkwy 8B WB & 0 & $\begin{array}{l}\text { Right Turn } \\
\text { Through } \\
\text { Left Turn }\end{array}$ & & $\begin{array}{l}0 \\
0 \\
0\end{array}$ \\
\hline & FH Pkwy 8C EB & 0 & $\begin{array}{l}\text { Right Turn } \\
\text { Through } \\
\text { Left Turn }\end{array}$ & & $\begin{array}{l}0 \\
0 \\
0\end{array}$ \\
\hline & SR 416 SB & 142 & $\begin{array}{l}\text { Right Turn } \\
\text { Through } \\
\text { Left Turn }\end{array}$ & $\begin{array}{r}0.00 \% \\
100.00 \% \\
0.00 \%\end{array}$ & $\begin{array}{r}0 \\
142 \\
0\end{array}$ \\
\hline & SR 416 NB & 115 & $\begin{array}{l}\text { Right Turn } \\
\text { Through } \\
\text { Left Turn }\end{array}$ & $\begin{array}{r}0.00 \% \\
100.00 \% \\
0.00 \% \\
\end{array}$ & $\begin{array}{r}0 \\
115 \\
0 \\
\end{array}$ \\
\hline
\end{tabular}


Table K.62. Weekday P.M. peak-hour intersection volume projections for 2026 - no build alternative

\begin{tabular}{|c|c|c|c|c|c|}
\hline \multirow[b]{2}{*}{ Intersection location } & \multirow[b]{2}{*}{ Approach } & \multirow{2}{*}{$\begin{array}{c}\text { Total } \\
\text { vehicles } \\
\text { per hour }\end{array}$} & \multicolumn{3}{|c|}{ Directional information } \\
\hline & & & $\begin{array}{c}\text { Turning } \\
\text { movement }\end{array}$ & Percent & Counts \\
\hline \multirow[t]{4}{*}{ U.S. 321 Intersection w/ Foothills Pkwy } & FH Pkwy 8A WB & 202 & $\begin{array}{l}\text { Right Tum } \\
\text { Through } \\
\text { Left Tum }\end{array}$ & $\begin{array}{r}16.34 \% \\
0.00 \% \\
83.66 \%\end{array}$ & $\begin{array}{r}33 \\
0 \\
169\end{array}$ \\
\hline & FH Pkwy 8B EB & 0 & $\begin{array}{l}\text { Right Turn } \\
\text { Through } \\
\text { Left Turn }\end{array}$ & & $\begin{array}{l}0 \\
0 \\
0\end{array}$ \\
\hline & U.S. $321 \mathrm{SB}$ & 711 & $\begin{array}{l}\text { Right Tum } \\
\text { Through } \\
\text { Left Turn }\end{array}$ & $\begin{array}{r}0.00 \% \\
97.33 \% \\
2.67 \%\end{array}$ & $\begin{array}{r}0 \\
692 \\
19\end{array}$ \\
\hline & U.S. $321 \mathrm{NB}$ & 936 & $\begin{array}{l}\text { Right Tum } \\
\text { Through } \\
\text { Left Tum }\end{array}$ & $\begin{array}{r}13.03 \% \\
86.97 \% \\
0.00 \%\end{array}$ & $\begin{array}{r}122 \\
814 \\
0\end{array}$ \\
\hline \multirow[t]{3}{*}{ U.S. 321 Convergence w/SR 32} & U.S. $321 \mathrm{~EB}$ & 817 & $\begin{array}{l}\text { Right Tum } \\
\text { Left Tum }\end{array}$ & $\begin{array}{l}12.61 \% \\
87.39 \%\end{array}$ & $\begin{array}{l}103 \\
714\end{array}$ \\
\hline & U.S. $321 \mathrm{SB}$ & 341 & $\begin{array}{l}\text { Right Tum } \\
\text { Through }\end{array}$ & $\begin{array}{l}78.30 \% \\
21.70 \%\end{array}$ & $\begin{array}{r}267 \\
74\end{array}$ \\
\hline & SR $32 \mathrm{NB}$ & 64 & $\begin{array}{l}\text { Through } \\
\text { Left Turn }\end{array}$ & $\begin{array}{l}62.50 \% \\
37.50 \%\end{array}$ & $\begin{array}{l}40 \\
24\end{array}$ \\
\hline \multirow[t]{3}{*}{ U.S. 321 Intersection $w / S R 416$} & SR 416 SB & 115 & $\begin{array}{l}\text { Right Turn } \\
\text { Left Turn }\end{array}$ & $\begin{array}{l}84.35 \% \\
15.65 \%\end{array}$ & $\begin{array}{l}97 \\
18\end{array}$ \\
\hline & U.S. 321 WB & 1018 & $\begin{array}{l}\text { Right Tum } \\
\text { Through }\end{array}$ & $\begin{array}{r}3.83 \% \\
96.17 \%\end{array}$ & $\begin{array}{r}39 \\
979\end{array}$ \\
\hline & U.S. $321 \mathrm{~EB}$ & 459 & $\begin{array}{l}\text { Through } \\
\text { Left Tum }\end{array}$ & $\begin{array}{r}94.77 \% \\
5.23 \%\end{array}$ & $\begin{array}{r}435 \\
24\end{array}$ \\
\hline \multirow[t]{3}{*}{ SR 416 Intersection w/ Webb Creek Rd } & Webb Cr Rd WB & 89 & $\begin{array}{l}\text { Right Turn } \\
\text { Left Turn }\end{array}$ & $\begin{array}{r}6.74 \% \\
93.26 \%\end{array}$ & $\begin{array}{r}6 \\
83\end{array}$ \\
\hline & SR $416 \mathrm{SB}$ & 80 & $\begin{array}{l}\text { Through } \\
\text { Left Turn }\end{array}$ & $\begin{array}{r}98.75 \% \\
1.25 \%\end{array}$ & $\begin{array}{r}79 \\
1\end{array}$ \\
\hline & SR $416 \mathrm{NB}$ & 148 & $\begin{array}{l}\text { Right Tum } \\
\text { Through }\end{array}$ & $\begin{array}{l}44.59 \% \\
55.41 \%\end{array}$ & $\begin{array}{l}66 \\
82\end{array}$ \\
\hline \multirow[t]{4}{*}{ SR 416 Intersection w/ Foothills Pkwy 8B/8C } & FH Pkwy 8B WB & 0 & $\begin{array}{l}\text { Right Tum } \\
\text { Through } \\
\text { Left Tum }\end{array}$ & & $\begin{array}{l}0 \\
0 \\
0\end{array}$ \\
\hline & FH Pkwy 8C EB & 0 & $\begin{array}{l}\text { Right Turn } \\
\text { Through } \\
\text { Left Tum }\end{array}$ & & $\begin{array}{l}0 \\
0 \\
0\end{array}$ \\
\hline & SR 416 SB & 163 & $\begin{array}{l}\text { Right Turn } \\
\text { Through } \\
\text { Left Tum }\end{array}$ & $\begin{array}{r}0.00 \% \\
100.00 \% \\
0.00 \%\end{array}$ & $\begin{array}{r}0 \\
163 \\
0\end{array}$ \\
\hline & SR 416 NB & 149 & $\begin{array}{l}\text { Right Turn } \\
\text { Through } \\
\text { Left Turn }\end{array}$ & $\begin{array}{r}0.00 \% \\
100.00 \% \\
0.00 \%\end{array}$ & $\begin{array}{r}0 \\
149 \\
0\end{array}$ \\
\hline
\end{tabular}


Table K.63. Weekend A.M. peak-hour intersection volume projections for $2026-$ no build alternative

\begin{tabular}{|c|c|c|c|c|c|}
\hline \multirow[b]{2}{*}{ Intersection location } & \multirow[b]{2}{*}{ Approach } & \multirow{2}{*}{$\begin{array}{c}\text { Total } \\
\text { vehicles } \\
\text { per hour }\end{array}$} & \multicolumn{3}{|c|}{ Directional information } \\
\hline & & & $\begin{array}{c}\text { Turning } \\
\text { movement }\end{array}$ & Percent & Counts \\
\hline \multirow[t]{4}{*}{ U.S. 321 Intersection w/ Foothills Pkwy } & FH Pkwy 8A WB & 463 & $\begin{array}{l}\text { Right Tum } \\
\text { Through } \\
\text { Left Tum }\end{array}$ & $\begin{array}{r}13.17 \% \\
0.00 \% \\
86.83 \%\end{array}$ & $\begin{array}{r}61 \\
0 \\
402\end{array}$ \\
\hline & FH Pkwy 8B EB & 0 & $\begin{array}{l}\text { Right Turn } \\
\text { Through } \\
\text { Left Turn }\end{array}$ & $\begin{array}{l}0.00 \% \\
0.00 \% \\
0.00 \%\end{array}$ & $\begin{array}{l}0 \\
0 \\
0\end{array}$ \\
\hline & U.S. $321 \mathrm{SB}$ & 749 & $\begin{array}{l}\text { Right Turn } \\
\text { Through } \\
\text { Left Turn }\end{array}$ & $\begin{array}{r}0.00 \% \\
91.32 \% \\
8.68 \%\end{array}$ & $\begin{array}{r}0 \\
684 \\
65\end{array}$ \\
\hline & U.S. $321 \mathrm{NB}$ & 1126 & $\begin{array}{l}\text { Right Tum } \\
\text { Through } \\
\text { Left Turn }\end{array}$ & $\begin{array}{r}19.98 \% \\
80.02 \% \\
0.00 \%\end{array}$ & $\begin{array}{r}225 \\
901 \\
0\end{array}$ \\
\hline \multirow[t]{3}{*}{ U.S. 321 Convergence w/ SR 32} & U.S. $321 \mathrm{~EB}$ & 846 & $\begin{array}{l}\text { Right Tum } \\
\text { Left Turn }\end{array}$ & $\begin{array}{l}12.41 \% \\
87.59 \%\end{array}$ & $\begin{array}{l}105 \\
741\end{array}$ \\
\hline & U.S. $321 \mathrm{SB}$ & 775 & $\begin{array}{l}\text { Right Turn } \\
\text { Through }\end{array}$ & $\begin{array}{l}77.81 \% \\
22.19 \%\end{array}$ & $\begin{array}{l}603 \\
172\end{array}$ \\
\hline & SR $32 \mathrm{NB}$ & 123 & $\begin{array}{l}\text { Through } \\
\text { Left Turn }\end{array}$ & $\begin{array}{l}65.85 \% \\
34.15 \%\end{array}$ & $\begin{array}{l}81 \\
42\end{array}$ \\
\hline \multirow[t]{3}{*}{ U.S. 321 Intersection w/ SR 416} & SR $416 \mathrm{SB}$ & 191 & $\begin{array}{l}\text { Right Tum } \\
\text { Left Tum }\end{array}$ & $\begin{array}{l}81.68 \% \\
18.32 \%\end{array}$ & $\begin{array}{r}156 \\
35\end{array}$ \\
\hline & U.S. 321 WB & 1280 & $\begin{array}{l}\text { Right Turn } \\
\text { Through }\end{array}$ & $\begin{array}{r}1.80 \% \\
98.20 \%\end{array}$ & $\begin{array}{r}23 \\
1257\end{array}$ \\
\hline & U.S. $321 \mathrm{~EB}$ & 695 & $\begin{array}{l}\text { Through } \\
\text { Left Turn }\end{array}$ & $\begin{array}{r}91.80 \% \\
8.20 \%\end{array}$ & $\begin{array}{r}638 \\
57\end{array}$ \\
\hline \multirow[t]{3}{*}{ SR 416 Intersection $w /$ Webb Creek Rd } & Webb Cr Rd WB & 52 & $\begin{array}{l}\text { Right Turn } \\
\text { Left Turn }\end{array}$ & $\begin{array}{r}3.85 \% \\
96.15 \%\end{array}$ & $\begin{array}{r}2 \\
50\end{array}$ \\
\hline & SR 416 SB & 48 & $\begin{array}{l}\text { Through } \\
\text { Left Turn }\end{array}$ & $\begin{array}{r}95.83 \% \\
4.17 \%\end{array}$ & $\begin{array}{r}46 \\
2\end{array}$ \\
\hline & SR $416 \mathrm{NB}$ & 96 & $\begin{array}{l}\text { Right Tum } \\
\text { Through }\end{array}$ & $\begin{array}{l}47.92 \% \\
52.08 \%\end{array}$ & $\begin{array}{l}46 \\
50\end{array}$ \\
\hline \multirow[t]{4}{*}{ SR 416 Intersection w/ Foothills Pkwy $8 \mathrm{~B} / 8 \mathrm{C}$} & FH Pkwy 8B WB & 0 & $\begin{array}{l}\text { Right Tum } \\
\text { Through } \\
\text { Left Turn }\end{array}$ & $\begin{array}{l}0.00 \% \\
0.00 \% \\
0.00 \%\end{array}$ & $\begin{array}{l}0 \\
0 \\
0\end{array}$ \\
\hline & FH Pkwy 8C EB & 0 & $\begin{array}{l}\text { Right Turn } \\
\text { Through } \\
\text { Left Turn }\end{array}$ & $\begin{array}{l}0.00 \% \\
0.00 \% \\
0.00 \%\end{array}$ & $\begin{array}{l}0 \\
0 \\
0\end{array}$ \\
\hline & SR $416 \mathrm{SB}$ & 96 & $\begin{array}{l}\text { Right Turn } \\
\text { Through } \\
\text { Left Turn }\end{array}$ & $\begin{array}{r}0.00 \% \\
100.00 \% \\
0.00 \%\end{array}$ & $\begin{array}{r}0 \\
96 \\
0\end{array}$ \\
\hline & SR $416 \mathrm{NB}$ & 96 & $\begin{array}{l}\text { Right Turn } \\
\text { Through } \\
\text { Left Tum }\end{array}$ & $\begin{array}{r}0.00 \% \\
100.00 \% \\
0.00 \%\end{array}$ & $\begin{array}{r}0 \\
96 \\
0\end{array}$ \\
\hline
\end{tabular}


Table K.64. Weekend P.M. peak-hour intersection volume projections for 2026-no build alternative

\begin{tabular}{|c|c|c|c|c|c|}
\hline \multirow[b]{2}{*}{ Intersection location } & \multirow[b]{2}{*}{ Approach } & \multirow{2}{*}{$\begin{array}{c}\text { Total } \\
\text { vehicles } \\
\text { per hout }\end{array}$} & \multicolumn{3}{|c|}{ Directional information } \\
\hline & & & $\begin{array}{c}\text { Turning } \\
\text { movement }\end{array}$ & Percent & Counts \\
\hline \multirow[t]{12}{*}{ U.S. 321 Intersection w/ Foothills Pkwy } & FH Pkwy 8A WB & 283 & Right Turn & $16.61 \%$ & 47 \\
\hline & & & Through & $0.00 \%$ & 0 \\
\hline & & & Left Tum & $83.39 \%$ & 236 \\
\hline & FH Pkwy 8B EB & 0 & Right Turn & & 0 \\
\hline & & & Through & & 0 \\
\hline & & & Left Turn & & 0 \\
\hline & U.S. $321 \mathrm{SB}$ & 833 & Right Turn & $0.00 \%$ & 0 \\
\hline & & & Through & $97.36 \%$ & 811 \\
\hline & & & Left Turn & $2.64 \%$ & 22 \\
\hline & U.S. $321 \mathrm{NB}$ & 1069 & Right Tum & $12.91 \%$ & 138 \\
\hline & & & Through & $87.09 \%$ & 931 \\
\hline & & & Left Turn & $0.00 \%$ & 0 \\
\hline \multirow[t]{6}{*}{ U.S. 321 Convergence w/ SR 32} & U.S. $321 \mathrm{~EB}$ & 985 & Right Turn & $12.79 \%$ & 126 \\
\hline & & & Left Turn & $87.21 \%$ & 859 \\
\hline & U.S. $321 \mathrm{SB}$ & 647 & Right Turn & $78.05 \%$ & 505 \\
\hline & & & Through & $21.95 \%$ & 142 \\
\hline & SR 32 NB & 83 & Through & $61.45 \%$ & 51 \\
\hline & & & Left Turn & $38.55 \%$ & 32 \\
\hline \multirow[t]{6}{*}{ U.S. 321 Intersection w/ SR 416} & SR $416 \mathrm{SB}$ & 224 & Right Tum & $83.48 \%$ & 187 \\
\hline & & & Left Tum & $16.52 \%$ & 37 \\
\hline & U.S. $321 \mathrm{WB}$ & 1362 & Right Turn & $3.89 \%$ & 53 \\
\hline & & & Through & $96.11 \%$ & 1309 \\
\hline & U.S. $321 \mathrm{~EB}$ & 654 & Through & $94.80 \%$ & 620 \\
\hline & & & Left Turn & $5.20 \%$ & 34 \\
\hline \multirow[t]{6}{*}{ SR 416 Intersection w/ Webb Creek Rd } & Webb Cr Rd WB & 68 & Right Turn & $7.35 \%$ & 5 \\
\hline & & & Left Turn & $92.65 \%$ & 63 \\
\hline & SR 416 SB & 110 & Through & $99.09 \%$ & 109 \\
\hline & & & Left Turn & $0.91 \%$ & 1 \\
\hline & SR $416 \mathrm{NB}$ & 148 & Right Turn & $44.59 \%$ & 66 \\
\hline & & & Through & $55.41 \%$ & 82 \\
\hline \multirow[t]{12}{*}{ SR 416 Intersection w/ Foothills Pkwy 8B/8C } & FH Pkwy 8B WB & 0 & Right Tum & & 0 \\
\hline & & & Through & & 0 \\
\hline & & & Left Turn & & 0 \\
\hline & FH Pkwy 8C EB & 0 & Right Turn & & 0 \\
\hline & & & Through & & 0 \\
\hline & & & Left Tum & & 0 \\
\hline & SR 416 SB & 171 & Right Turn & $0.00 \%$ & 0 \\
\hline & & & Through & $100.00 \%$ & 171 \\
\hline & & & Left Turn & $0.00 \%$ & 0 \\
\hline & SR 416 NB & 149 & Right Turn & $0.00 \%$ & 0 \\
\hline & & & Through & $100.00 \%$ & 149 \\
\hline & & & Left Tum & $0.00 \%$ & 0 \\
\hline
\end{tabular}




\title{
Appendix L
}

\section{NOISE DATA}

\author{
S. M. Chin \\ Oak Ridge National Laboratory \\ Oak Ridge, Tennessee \\ R. Gibson \\ Oak Ridge National Laboratory \\ Oak Ridge, Tennessee
}

August 1995 



\section{L.1 CONSTRUCT SECTIONS 8B AND 8C WITH NO INTERCHANGES}

The opening on Sections 8B and 8C would divert tourist related traffic from U.S. 321 to Foothills Parkway. The Foothills Parkway traffic would be light and most of the U.S. 321 traffic would stay on U.S. 321. Similar to the option described above, most sites would encounter little traffic noise impacts and would experience noise levels within the standard for residential areas. In 2006, the highest $L_{e q}$ noise level at 34 sites would be below $50 \mathrm{dBA}$; no sites would experience levels between 50-60 dBA; and 5 sites would experience levels between 60-67 dBA. In 2026, the highest $\mathrm{L}_{\text {eq }}$ noise level at 34 sites would be below $50 \mathrm{dBA}$; and 5 sites would experience levels between 60-67 dBA. Noise levels at 2 sites (sites 6 and 19) would exceed standards by 2006. The addition of Section $8 \mathrm{~B}$ would slightly decrease the traffic volume along U.S. 321, thereby decreasing the projected noise levels for sites 6 and 19.

Sites $16,17,18,25,26,27$ and 40 would experience significant increases in noise level, as much as $12 \mathrm{dBA}$ during some peak periods, compared to the no-build alternative. The significant increases in noise levels at these sites are due to the low existing noise levels. The projected noise levels for these sites are still low, ranging from 30 to $42 \mathrm{dBA}$. It should be noted that the existing noise levels measured at these sites are higher than the projected traffic noise levels. This suggests that ambient noise other than traffic noise dominates the noise level at these sites.

\section{L.2 FOOTHILLS PARKWAY INTERCHANGE AT SR 416 WITH NORTH RAMP OR SOUTH RAMP}

Under these build options, some of the traffic on Sections 8B and 8C will use SR 416. However, Parkway-related traffic on SR 416 would be very low, and the noise impact would be similar to the no interchange option. That is, most sites would encounter little traffic noise impacts, even by 2026 , and would experience noise levels within the standard for residential areas. The highest $\mathrm{L}_{\text {eq }}$ noise level at 34 sites would still be below $50 \mathrm{dBA}$ by 2026; and 5 sites would experience levels between 60-67 dBA. However, all sites along SR 416 would experience noise levels below the FHWA-established standard. Noise levels at sites 6 and 19 along U.S. 321 would exceed standards by 2006 .

Sites $16,17,18,25,26,27$, and 40 would experience significant increases in noise level, as much as $12 \mathrm{dBA}$ during some peak periods, compared to the no-build alternative. These increases are identical for those resulting from the no interchange option. The significant increases in noise levels at these sites are due to the low existing noise levels. The projected noise levels for these sites are still low, ranging from 30 to $42 \mathrm{dBA}$, and fall well below the FHWA-established standard for residential areas. As indicated, the existing noise levels measured at these sites are higher than the projected traffic noise level. This indicates that other ambient noise rather than traffic noise dominates the noise level at these sites.

\section{L.3 FOOTHILLS PARKWAY INTERCHANGE AT U.S. 321 WITH TWO DIFFERENT RAMP CONNECTION CONFIGURATION OPTIONS}

These build options are very similar to the SR 416 interchange option except that the Foothills Parkway/Pittman Center interchange would be at U.S. 321 instead of at SR 416. Some of the Section $8 \mathrm{~B}$ and $8 \mathrm{C}$ traffic would use U.S. 321 directly, but such traffic would be very light. Similar to the previously described options, most sites would encounter little traffic noise impacts 
and would experience noise levels within the standard for residential areas. By 2026 the highest $\mathrm{L}_{\text {eq }}$ noise level at 34 sites would still be below $50 \mathrm{dBA}$; and 5 sites would experience levels between 60-67 dBA. Noise levels at sites 6 and 19, however, would exceed FHWA standards by 2006.

Sites $16,17,18,25,26,27$, and 40 would experience significant increases in noise level, as much as $12 \mathrm{dBA}$ during some peak periods, compared to the no-build alternative. These increases are identical for those resulting from the SR 416 interchange option. The significant increases in noise levels at these sites are due to the low existing noise levels. The projected noise levels for these sites are low, ranging from 30 to $42 \mathrm{dBA}$, and fall well below the FHWA-established standard for residential areas. As indicated, the existing noise level measured at these sites is higher than the projected traffic noise. This indicates that ambient noise other than traffic noise dominates the noise level at these sites.

\section{L.4 8B WOULD BE OPERATIONAL PRIOR TO THE COMPLETION SECTION 8C WITH INTERCHANGE AT SR 416}

Under this build option, all traffic on Section 8B must use SR 416 to enter and exit the Foothills Parkway and continue their trips on U.S. 321. Consequently, the noise levels at Site 10, in addition to Sites 6 and 19, near SR 416 would experience traffic noise impacts with noise levels exceeding FHWA residential noise standards by 2006 . Most other sites would encounter little traffic noise impacts and would experience noise levels within the standard for residential areas. The highest $\mathrm{L}_{\mathrm{eq}}$ noise level at 34 sites would be below $50 \mathrm{dBA}$, and 4 sites would experience levels between 60-67 dBA.

Sites $10,11,16,17,18,25,26,27$, and 40 would experience significant increases in noise level, as much as $12 \mathrm{dBA}$ during some peak periods, compared to the no-build alternative. The increase in noise levels at Sites 10 and 11 are due to the addition of Section $8 B$ traffic on SR 416. Noise level increases at the other sites are identical to those described for the SR 416 interchange option. The significant increases in noise levels at these sites are due to the low existing noise levels. Still, the projected noise levels for these sites are low, ranging from 30 to $42 \mathrm{dBA}$, and fall well below the FHWA-established standard for residential areas. As indicated, the existing noise levels measured at these sites are higher than the projected traffic noises. This indicates that ambient noise other than traffic noise dominates the noise level at these sites.

\section{L.5 8B WOULD BE OPERATIONAL PRIOR TO THE COMPLETION SECTION 8C WITH INTERCHANGE AT U.S. 321}

Under this build option, all traffic on Section 8B would use U.S. 321 directly to enter and exit the Foothills Parkway. No Foothills Parkway-related traffic would use SR 416. Thus, as in the U.S. 321 interchange options, most sites would encounter little traffic noise impacts and would experience noise levels within the standard for residential areas. The highest $\mathrm{L}_{\text {eq }}$ noise level at 34 sites would be below $50 \mathrm{dBA}$; and 5 sites would experience levels between $60-67 \mathrm{dBA}$. Noise levels at sites 6 and 19 would exceed standards by 2006 .

Sites $16,17,18,25,26,27$, and 40 would experience significant increases in noise levels, as much as $12 \mathrm{dBA}$ during some peak periods, compared to the no-build alternative. These increases would range from 3 to $10 \mathrm{dBA}$ for different peak time periods. The significant increases in traffic noise 
levels at these sites are due to the low existing noise levels. Still, the projected noise levels for these sites are low, ranging from 30 to $42 \mathrm{dBA}$, and fall well below the FHWA-established standard for residential areas. As indicated, the existing noise levels measured at these sites are higher than the projected traffic noises. This indicates that other ambient noise rather than traffic noise dominates the noise level at these sites.

\section{L.6 NO-ACTION ALTERNATIVE (NO-BUILD)}

ORNL's noise projections for the study area indicate that, for this build option, most sites would encounter little traffic noise impacts and would experience traffic noise levels below the FHWAestablished standard for residential areas through the year 2026. Most sites (34 sites) have a projected noise level of less than $50 \mathrm{dBA}$ during the noisiest peak period in both 2006 and 2026; 2 sites have levels 50-60; and only 3 sites have levels between 60 and $67 \mathrm{dBA}$.

Two sites along U.S. 321 would experience noise levels that exceed this standard by the year 2006 due to their close proximity to the highway and the large volume of traffic expected to travel along it. The projected $L_{e q}$ noise levels at these sites (i.e., sites 6 and 19) would range from 70 to $73 \mathrm{dBA}$ by 2006 and from 70 to $75 \mathrm{dBA}$ by 2026 . 
Table L.1. Noise level projections-construct 8B with no interchanges option

\begin{tabular}{|c|c|c|c|c|c|c|c|c|c|c|}
\hline \multirow{4}{*}{$\begin{array}{l}\text { Site } \\
\text { no. }\end{array}$} & \multirow[b]{4}{*}{ Location } & \multicolumn{9}{|c|}{ Noise level (Leq) } \\
\hline & & \multirow[b]{3}{*}{ Existing } & \multicolumn{4}{|c|}{2006} & \multicolumn{4}{|c|}{2026} \\
\hline & & & \multicolumn{2}{|c|}{ Weekday } & \multicolumn{2}{|c|}{ Weekend } & \multicolumn{2}{|c|}{ Weekday } & \multicolumn{2}{|c|}{ Weekend } \\
\hline & & & A.M. & P.M. & A.M. & P.M. & A.M. & P.M. & A.M. & P.M. \\
\hline 1 & $\begin{array}{l}\text { Foothills Pkwy Sec. } 8 \mathrm{~A}-1 \text { st Scenic } \\
\text { Overlook from U.S. } 321\end{array}$ & 47.3 & 59 & 58 & 61 & 59 & 61 & 59 & 62 & 61 \\
\hline 2 & $\begin{array}{l}\text { Foothills Pkwy Sec. 8A-1st Scenic } \\
\text { Overlook from I-40 }\end{array}$ & 47.3 & 59 & 58 & 61 & 59 & 61 & 59 & 62 & 61 \\
\hline 3 & $\begin{array}{l}\text { Burnett Memorial Methodist } \\
\text { Church-Pittman Center }\end{array}$ & 49.0 & 43 & 43 & 43 & 43 & 45 & 45 & 45 & 45 \\
\hline 4 & $\begin{array}{l}\text { Pittman Center Elementary } \\
\text { School-Playground }\end{array}$ & 42.6 & 43 & 43 & 43 & 43 & 45 & 45 & 45 & 45 \\
\hline 5 & Pittman Center City Hall-Parking lot & 51.3 & 45 & 45 & 46 & 46 & 47 & 47 & 47 & 48 \\
\hline 6 & $\begin{array}{l}\text { House \#3355 between Mile Markers } 20 \text { and } \\
21 \text { on. U.S. } 321 \text {-Front yard }\end{array}$ & 67.2 & 71 & 71 & 73 & 73 & 73 & 73 & 74 & 74 \\
\hline 7 & House \#5106 on SR 416-Front yard & 47.7 & 43 & 43 & 43 & 43 & 45 & 45 & 45 & 45 \\
\hline 8 & $\begin{array}{l}\text { House \#3010 on Webb Creek Road-Front } \\
\text { yard }\end{array}$ & 40.7 & 43 & 43 & 43 & 43 & 45 & 45 & 45 & 45 \\
\hline 9 & House \#5005 on SR 416-Front yard & 62.4 & 43 & 43 & 43 & 43 & 45 & 45 & 45 & 45 \\
\hline 10 & House \#5215 on SR 416-Front yard & 49.7 & 65 & 65 & 66 & 66 & 66 & 66 & 66 & 67 \\
\hline 11 & House \#5328 on SR 416-Front yard & 54.3 & 60 & 60 & 60 & 61 & 60 & 60 & 60 & 62 \\
\hline 12 & $\begin{array}{l}\text { Greenbrier View Rental Cottage on Tunis } \\
\text { Branch Road }\end{array}$ & 39.4 & 35 & 35 & 35 & 35 & 37 & 38 & 38 & 38 \\
\hline 13 & $\begin{array}{l}\text { House \#209 on Teaberry Hill Road-Front } \\
\text { yard }\end{array}$ & 39.1 & 45 & 45 & 45 & 45 & 47 & 47 & 47 & 47 \\
\hline 14 & $\begin{array}{l}\text { House \#4705 on Townsend Road-Front } \\
\text { yard }\end{array}$ & 45.8 & 40 & 41 & 42 & 42 & 42 & 42 & 43 & 43 \\
\hline 15 & $\begin{array}{l}\text { House at End of Branam Hollow } \\
\text { Road-Back yard of guest house }\end{array}$ & 38.7 & 38 & 38 & 38 & 38 & 40 & 40 & 40 & 40 \\
\hline 16 & $\begin{array}{l}\text { House \#612 on Branam Hollow } \\
\text { Road-Driveway }\end{array}$ & 35.5 & 30 & 31 & 31 & 31 & 32 & 32 & 33 & 33 \\
\hline 17 & $\begin{array}{l}\text { House \#5258 Off of Mathis Branch } \\
\text { Road-Front yard }\end{array}$ & 53.2 & 28 & 28 & 29 & 29 & 29 & 30 & 30 & 31 \\
\hline 18 & House Off of Rocky Flatts Road-Back yard & 64.0 & 39 & 39 & 40 & 40 & 41 & 41 & 42 & 42 \\
\hline 19 & House \#5039 on U.S. 321-Front yard & 63.1 & 69 & 69 & 71 & 71 & 71 & 71 & 72 & 72 \\
\hline 20 & House \#4022 on U.S. 321-Back yard & 53.6 & 43 & 43 & 43 & 43 & 45 & 45 & 45 & 45 \\
\hline 21 & Young's Taxedermy Off U.S 321-Driveway & 51.8 & 58 & 59 & 60 & 60 & 60 & 60 & 61 & 61 \\
\hline 22 & House \#4140 on Chavis Road-Front yard & 49.3 & 45 & 45 & 45 & 45 & 48 & 48 & 48 & 48 \\
\hline
\end{tabular}




\begin{tabular}{|c|c|c|c|c|c|c|c|c|c|c|}
\hline \multirow{4}{*}{$\begin{array}{l}\text { Site } \\
\text { no. }\end{array}$} & \multirow[b]{4}{*}{ Location } & \multicolumn{9}{|c|}{ Noise level (Leq) } \\
\hline & & \multirow[b]{3}{*}{ Existing } & \multicolumn{4}{|c|}{2006} & \multicolumn{4}{|c|}{2026} \\
\hline & & & \multicolumn{2}{|c|}{ Weekday } & \multicolumn{2}{|c|}{ Weekend } & \multicolumn{2}{|c|}{ Weekday } & \multicolumn{2}{|c|}{ Weekend } \\
\hline & & & A.M. & P.M. & A.M. & P.M. & A.M. & P.M. & A.M. & P.M. \\
\hline 23 & Waters-Large Cemetery-Entrance & 37.6 & 45 & 45 & 46 & 46 & 46 & 46 & 47 & 47 \\
\hline 24 & House \#227 Teaberry Lane & 42.0 & 40 & 40 & 42 & 42 & 42 & 42 & 43 & 43 \\
\hline 25 & $\begin{array}{l}\text { House \#5310 on Otto Williams Road-Back } \\
\text { yard }\end{array}$ & 52.4 & 36 & 37 & 37 & 38 & 38 & 38 & 39 & 39 \\
\hline 26 & House \#327 on Costner Road-Back yard & 44.4 & 35 & 36 & 36 & 36 & 37 & 38 & 38 & 38 \\
\hline 27 & House \#430 Appletree Lane-Front yard & 40.3 & 29 & 29 & 30 & 30 & 31 & 31 & 32 & 32 \\
\hline 28 & $\begin{array}{l}\text { House on Sunshine Trail in Cobbly } \\
\text { Knob-Baicony }\end{array}$ & 41.8 & 33 & 33 & 33 & 34 & 35 & 35 & 36 & 36 \\
\hline 29 & Rental House on McKinzi Way-Front yard & 39.7 & 43 & 43 & 43 & 43 & 45 & 45 & 45 & 45 \\
\hline 30 & House on Shultz Road-Driveway & 39.6 & 43 & 43 & 43 & 43 & 45 & 45 & 45 & 45 \\
\hline 31 & Copeland Rental Cabins-Copeland Road & 47.1 & 43 & 43 & 43 & 43 & 45 & 45 & 45 & 45 \\
\hline 32 & House on The Way (last house)-Front yard & 41.1 & 43 & 43 & 44 & 44 & 45 & 45 & 46 & 46 \\
\hline 33 & $\begin{array}{l}\text { House on The Way (second to last } \\
\text { house)-Front deck }\end{array}$ & 45.6 & 41 & 41 & 42 & 43 & 43 & 43 & 44 & 44 \\
\hline 34 & House \#429 on Appletree Lane & 50.8 & 46 & 46 & 46 & 46 & 49 & 49 & 49 & 49 \\
\hline 35 & $\begin{array}{l}\text { House at Intersection of Waters Cemetery } \\
\text { Road and Chavis Road }\end{array}$ & 49.5 & 39 & 39 & 40 & 40 & 42 & 42 & 42 & 42 \\
\hline 36 & House Off of Shultz Road & 46.9 & 45 & 45 & 47 & 47 & 47 & 47 & 48 & 48 \\
\hline 37 & $\begin{array}{l}\text { House \#916 Scenic Trail (Whistler's Watch) } \\
\text { in Cobbly Knob-Driveway }\end{array}$ & 41.3 & 41 & 41 & 41 & 41 & 43 & 43 & 43 & 43 \\
\hline 38 & $\begin{array}{l}\text { House on Sutton Place (Hidden Haven) in } \\
\text { Cobbly Knob-Driveway }\end{array}$ & 35.1 & 32 & 33 & 33 & 33 & 35 & 35 & 35 & 35 \\
\hline 39 & $\begin{array}{l}\text { Gateway Near House on Black Gum Gap } \\
\text { Road in Cobbly Knob }\end{array}$ & 54.4 & 42 & 42 & 42 & 42 & 44 & 44 & 44 & 44 \\
\hline 40 & $\begin{array}{l}\text { House (under construction) at the End of } \\
\text { Squirrel Trail in Cobbly Knob-Near } \\
\text { driveway }\end{array}$ & 36.6 & 33 & 33 & 33 & 34 & 35 & 35 & 35 & 36 \\
\hline 41 & $\begin{array}{l}\text { House (uninhabited or under construction) on } \\
\text { Rocky Flatts Road }\end{array}$ & 48.4 & 44 & 44 & 44 & 44 & 46 & 46 & 46 & 46 \\
\hline
\end{tabular}


Table L.2. Noise level projections-interchange at Highway 416 (both options)-8B not opened until 8C completed

\begin{tabular}{|c|c|c|c|c|c|c|c|c|c|c|}
\hline \multirow{4}{*}{$\begin{array}{l}\text { Site } \\
\text { no. }\end{array}$} & \multirow[b]{4}{*}{ Location } & \multicolumn{9}{|c|}{ Noise level (Leq) } \\
\hline & & \multirow[b]{3}{*}{ Existing } & \multicolumn{4}{|c|}{2006} & \multicolumn{4}{|c|}{2026} \\
\hline & & & \multicolumn{2}{|c|}{ Weekday } & \multicolumn{2}{|c|}{ Weekend } & \multicolumn{2}{|c|}{ Weekday } & \multicolumn{2}{|c|}{ Weekend } \\
\hline & & & A.M. & P.M. & A.M. & P.M. & A.M. & P.M. & A.M. & P.M. \\
\hline 1 & $\begin{array}{l}\text { Foothills Pkwy Sec. } 8 \mathrm{~A}-1 \text { st Scenic } \\
\text { Overlook from U.S. } 321\end{array}$ & 47.3 & 59 & 58 & 61 & 59 & 61 & 59 & 62 & 61 \\
\hline 2 & $\begin{array}{l}\text { Foothills Pkwy Sec. 8A-1st Scenic } \\
\text { Overlook from I-40 }\end{array}$ & 47.3 & 59 & 58 & 61 & 59 & 61 & 59 & 62 & 61 \\
\hline 3 & $\begin{array}{l}\text { Burnett Memorial Methodist } \\
\text { Church-Pittman Center }\end{array}$ & 49.0 & 43 & 43 & 43 & 43 & 45 & 45 & 45 & 45 \\
\hline 4 & $\begin{array}{l}\text { Pittman Center Elementary } \\
\text { School-Playground }\end{array}$ & 42.6 & 43 & 43 & 43 & 43 & 45 & 45 & 45 & 45 \\
\hline 5 & Pittman Center City Hall-Parking lot & 51.3 & 46 & 46 & 46 & 47 & 47 & 47 & 47 & 48 \\
\hline 6 & $\begin{array}{l}\text { House \#3355 between Mile Markers } 20 \\
\text { and } 21 \text { on U.S. } 321 \text {-Front yard }\end{array}$ & 67.2 & 71 & 72 & 73 & 73 & 73 & 73 & 74 & 74 \\
\hline 7 & House $\# 5106$ on SR 416 -Front yard & 47.7 & 43 & 43 & 43 & 43 & 45 & 45 & 45 & 45 \\
\hline 8 & $\begin{array}{l}\text { House } \# 3010 \text { on Webb Creek } \\
\text { Road-Front yard }\end{array}$ & 40.7 & 43 & 43 & 43 & 43 & 45 & 45 & 45 & 45 \\
\hline 9 & House $\# 5005$ on SR $416-$ Front yard & 62.4 & 43 & 43 & 43 & 43 & 45 & 45 & 45 & 45 \\
\hline 10 & House $\# 5215$ on SR 416 -Front yard & 49.7 & 66 & 67 & 66 & 68 & 66 & 67 & 66 & 68 \\
\hline 11 & House $\# 5328$ on SR $416-$ Front yard & 54.3 & 60 & 61 & 60 & 62 & 60 & 61 & 60 & 62 \\
\hline 12 & $\begin{array}{l}\text { Greenbrier View Rental Cottage on Tunis } \\
\text { Branch Road }\end{array}$ & 39.4 & 35 & 35 & 35 & 35 & 37 & 38 & 38 & 38 \\
\hline 13 & $\begin{array}{l}\text { House } \# 209 \text { on Teaberry Hill } \\
\text { Road-Front yard }\end{array}$ & 39.1 & 45 & 45 & 45 & 46 & 47 & 47 & 47 & 47 \\
\hline 14 & $\begin{array}{l}\text { House } \$ 4705 \text { on Townsend Road-Front } \\
\text { yard }\end{array}$ & 45.8 & 41 & 41 & 42 & 42 & 42 & 42 & 43 & 43 \\
\hline 15 & $\begin{array}{l}\text { House at End of Branam Hollow } \\
\text { Road-Back yard of guest house }\end{array}$ & 38.7 & 38 & 38 & 38 & 38 & 40 & 40 & 40 & 40 \\
\hline 16 & $\begin{array}{l}\text { House } \# 612 \text { on Branam Hollow } \\
\text { Road-Driveway }\end{array}$ & 35.5 & 30 & 31 & 31 & 31 & 32 & 32 & 33 & 33 \\
\hline 17 & $\begin{array}{l}\text { House \#5258 Off of Mathis Branch } \\
\text { Road-Front yard }\end{array}$ & 53.2 & 28 & 28 & 29 & 29 & 29 & 30 & 30 & 31 \\
\hline 18 & $\begin{array}{l}\text { House Off of Rocky Flatts Road-Back } \\
\text { yard }\end{array}$ & 64.0 & 39 & 39 & 40 & 40 & 41 & 41 & 42 & 42 \\
\hline 19 & House $\# 5039$ on U.S. $321-$ Front yard & 63.1 & 69 & 70 & 71 & 71 & 71 & 71 & 72 & 72 \\
\hline 20 & House $\# 4022$ on U.S. $321-$ Back yard & 53.6 & 43 & 43 & 43 & 43 & 45 & 45 & 45 & 45 \\
\hline
\end{tabular}




\begin{tabular}{|c|c|c|c|c|c|c|c|c|c|c|}
\hline \multirow{4}{*}{$\begin{array}{l}\text { Site } \\
\text { no. }\end{array}$} & \multirow[b]{4}{*}{ Location } & \multicolumn{9}{|c|}{ Noise level (Leq) } \\
\hline & & \multirow[b]{3}{*}{ Existing } & \multicolumn{4}{|c|}{2006} & \multicolumn{4}{|c|}{2026} \\
\hline & & & \multicolumn{2}{|c|}{ Weekday } & \multicolumn{2}{|c|}{ Weekend } & \multicolumn{2}{|c|}{ Weekday } & \multicolumn{2}{|c|}{ Weekend } \\
\hline & & & A.M. & P.M. & A.M. & P.M. & A.M. & P.M. & A.M. & P.M. \\
\hline 21 & $\begin{array}{l}\text { Young's Taxedermy Off U.S } 321- \\
\text { Driveway }\end{array}$ & 51.8 & 59 & 59 & 60 & 60 & 60 & 60 & 61 & 61 \\
\hline 22 & $\begin{array}{l}\text { House } \# 4140 \text { on Chavis Road-Front } \\
\text { yard }\end{array}$ & 49.3 & 45 & 45 & 45 & 45 & 48 & 48 & 48 & 48 \\
\hline 23 & Waters-Large Cemetery-Entrance & 37.6 & 45 & 45 & 46 & 46 & 46 & 46 & 47 & 47 \\
\hline 24 & House \#227 Teaberry Lane & 42.0 & 40 & 41 & 42 & 42 & 42 & 42 & 43 & 43 \\
\hline 25 & $\begin{array}{l}\text { House } \# 5310 \text { on Otto Williams } \\
\text { Road-Back yard }\end{array}$ & 52.4 & 36 & 37 & 37 & 38 & 38 & 38 & 39 & 39 \\
\hline 26 & House \#327 on Costner Road-Back yard & 44.4 & 35 & 36 & 36 & 36 & 37 & 38 & 38 & 38 \\
\hline 27 & House \#430 Appletree Lane-Front yard & 40.3 & 29 & 29 & 30 & 30 & 31 & 31 & 32 & 32 \\
\hline 28 & $\begin{array}{l}\text { House on Sunshine Trail in Cobbly } \\
\text { Knob-Balcony }\end{array}$ & 41.8 & 33 & 33 & 33 & 34 & 35 & 35 & 36 & 36 \\
\hline 29 & $\begin{array}{l}\text { Rental House on McKinzi Way-Front } \\
\text { yard }\end{array}$ & 39.7 & 43 & 43 & 43 & 43 & 45 & 45 & 45 & 45 \\
\hline 30 & House on Shultz Road-Driveway & 39.6 & 43 & 43 & 43 & 43 & 45 & 45 & 45 & 45 \\
\hline 31 & Copeland Rental Cabins-Copeland Road & 47.1 & 43 & 43 & 43 & 43 & 45 & 45 & 45 & 45 \\
\hline 32 & $\begin{array}{l}\text { House on The Way (last house)-Front } \\
\text { yard }\end{array}$ & 41.1 & 43 & 43 & 44 & 44 & 45 & 45 & 46 & 46 \\
\hline 33 & $\begin{array}{l}\text { House on The Way (second to last } \\
\text { house)-Front deck }\end{array}$ & 45.6 & 41 & 42 & 42 & 43 & 42 & 43 & 44 & 44 \\
\hline 34 & House \#429 on Appletree Lane & 50.8 & 46 & 46 & 46 & 46 & 49 & 49 & 49 & 49 \\
\hline 35 & $\begin{array}{l}\text { House at Intersection of Waters Cemetery } \\
\text { Road and Chavis Road }\end{array}$ & 49.5 & 39 & 39 & 40 & 40 & 42 & 42 & 42 & 42 \\
\hline 36 & House Off of Shultz Road & 46.9 & 45 & 46 & 47 & 47 & 47 & 47 & 48 & 48 \\
\hline 37 & $\begin{array}{l}\text { House \#916 Scenic Trail (Whistler's } \\
\text { Watch) in Cobbly Knob-Driveway }\end{array}$ & 41.3 & 41 & 41 & 41 & 41 & 43 & 43 & 43 & 43 \\
\hline 38 & $\begin{array}{l}\text { House on Sutton Place (Hidden Haven) } \\
\text { in Cobbly Knob-Driveway }\end{array}$ & 35.1 & 32 & 33 & 33 & 33 & 35 & 35 & 35 & 35 \\
\hline 39 & $\begin{array}{l}\text { Gateway Near House on Black Gum Gap } \\
\text { Road in Cobbly Knob }\end{array}$ & 54.4 & 42 & 42 & 42 & 42 & 44 & 44 & 44 & 44 \\
\hline 40 & $\begin{array}{l}\text { House (under construction) at the End of } \\
\text { Squirrel Trail in Cobbly Knob-Near } \\
\text { driveway }\end{array}$ & 36.6 & 33 & 33 & 33 & 34 & 35 & 35 & 35 & 36 \\
\hline 41 & $\begin{array}{l}\text { House (uninhabited or under } \\
\text { construction) on Rocky Flatts Road }\end{array}$ & 48.4 & 44 & 44 & 44 & 44 & 46 & 46 & 46 & 46 \\
\hline
\end{tabular}


Table L.3. Noise level projections-interchange at U.S. 321 (both options) - 8B not opened until 8C completed

\begin{tabular}{|c|c|c|c|c|c|c|c|c|c|c|}
\hline \multirow{4}{*}{$\begin{array}{l}\text { Site } \\
\text { no. }\end{array}$} & \multirow[b]{4}{*}{ Location } & \multicolumn{9}{|c|}{ Noise level (Leq) } \\
\hline & & \multirow[b]{3}{*}{ Existing } & \multicolumn{4}{|c|}{2006} & \multicolumn{4}{|c|}{2026} \\
\hline & & & \multicolumn{2}{|c|}{ Weekday } & \multicolumn{2}{|c|}{ Weekend } & \multicolumn{2}{|c|}{ Weekday } & \multicolumn{2}{|c|}{ Weekend } \\
\hline & & & A.M. & P.M. & A.M. & P.M. & A.M. & P.M. & A.M. & P.M. \\
\hline 1 & $\begin{array}{l}\text { Foothills Pkwy Sec. 8A-1st Scenic } \\
\text { Overlook from U.S. } 321\end{array}$ & 47.3 & 59 & 58 & 61 & 59 & 61 & 59 & 62 & 61 \\
\hline 2 & $\begin{array}{l}\text { Foothills Pkwy Sec. } 8 \mathrm{~A}-1 \text { st Scenic } \\
\text { Overlook from I-40 }\end{array}$ & 47.3 & 59 & 58 & 61 & 59 & 61 & 59 & 62 & 61 \\
\hline 3 & $\begin{array}{l}\text { Burnett Memorial Methodist } \\
\text { Church-Pittman Center }\end{array}$ & 49.0 & 43 & 43 & 43 & 43 & 45 & 45 & 45 & 45 \\
\hline 4 & $\begin{array}{l}\text { Pittman Center Elementary } \\
\text { School-Playground }\end{array}$ & 42.6 & 43 & 43 & 43 & 43 & 45 & 45 & 45 & 45 \\
\hline 5 & Pittman Center City Hall-Parking lot & 51.3 & 45 & 45 & 46 & 46 & 47 & 47 & 47 & 48 \\
\hline 6 & $\begin{array}{l}\text { House \#3355 between Mile Markers } 20 \text { and } \\
21 \text { on U.S. } 321 \text {-Front yard }\end{array}$ & 67.2 & 71 & 71 & 73 & 73 & 73 & 73 & 74 & 74 \\
\hline 7 & House \#5106 on SR 416-Front yard & 47.7 & 43 & 43 & 43 & 43 & 45 & 45 & 45 & 45 \\
\hline 8 & $\begin{array}{l}\text { House \#3010 on Webb Creek Road-Front } \\
\text { yard }\end{array}$ & 40.7 & 43 & 43 & 43 & 43 & 45 & 45 & 45 & 45 \\
\hline 9 & House \#5005 on SR 416-Front yard & 62.4 & 43 & 43 & 43 & 43 & 45 & 45 & 45 & 45 \\
\hline 10 & House \#5215 on SR 416-Front yard & 49.7 & 65 & 65 & 66 & 67 & 66 & 66 & 66 & 67 \\
\hline 11 & House \#5328 on SR 416-Front yard & 54.3 & 60 & 60 & 60 & 61 & 60 & 60 & 60 & 62 \\
\hline 12 & $\begin{array}{l}\text { Greenbrier View Rental Cottage on Tunis } \\
\text { Branch Road }\end{array}$ & 39.4 & 35 & 35 & 35 & 35 & 37 & 38 & 38 & 38 \\
\hline 13 & $\begin{array}{l}\text { House \#209 on Teaberry Hill Road-Front } \\
\text { yard }\end{array}$ & 39.1 & 45 & 45 & 45 & 45 & 47 & 47 & 47 & 47 \\
\hline 14 & $\begin{array}{l}\text { House \#4705 on Townsend Road-Front } \\
\text { yard }\end{array}$ & 45.8 & 40 & 41 & 42 & 42 & 42 & 42 & 43 & 43 \\
\hline 15 & $\begin{array}{l}\text { House at End of Branam Hollow } \\
\text { Road-Back yard of guest house }\end{array}$ & 38.7 & 38 & 38 & 38 & 38 & 40 & 40 & 40 & 40 \\
\hline 16 & $\begin{array}{l}\text { House \#612 on Branam Hollow } \\
\text { Road-Driveway }\end{array}$ & 35.5 & 30 & 31 & 31 & 31 & 32 & 32 & 33 & 33 \\
\hline 17 & $\begin{array}{l}\text { House \#5258 Off of Mathis Branch } \\
\text { Road-Front yard }\end{array}$ & 53.2 & 28 & 28 & 29 & 29 & 29 & 30 & 30 & 31 \\
\hline 18 & House Off of Rocky Flatts Road-Back yard & 64.0 & 39 & 39 & 40 & 40 & 41 & 41 & 42 & 42 \\
\hline 19 & House $\# 5039$ on U.S. 321-Front yard & 63.1 & 69 & 69 & 71 & 71 & 71 & 71 & 72 & 72 \\
\hline 20 & House $\# 4022$ on U.S. $321-$ Back yard & 53.6 & 43 & 43 & 43 & 43 & 45 & 45 & 45 & 45 \\
\hline 21 & Young's Taxedermy Off U.S 321-Driveway & 51.8 & 58 & 59 & 60 & 60 & 60 & 60 & 61 & 61 \\
\hline
\end{tabular}


Table L.3. Continued

\begin{tabular}{|c|c|c|c|c|c|c|c|c|c|c|}
\hline \multirow{4}{*}{$\begin{array}{l}\text { Site } \\
\text { no. }\end{array}$} & \multirow{4}{*}{ Location } & \multicolumn{9}{|c|}{ Noise level (Leq) } \\
\hline & & \multirow[b]{3}{*}{ Existing } & \multicolumn{4}{|c|}{2006} & \multicolumn{4}{|c|}{2026} \\
\hline & & & \multicolumn{2}{|c|}{ Weekday } & \multicolumn{2}{|c|}{ Weekend } & \multicolumn{2}{|c|}{ Weekday } & \multicolumn{2}{|c|}{ Weekend } \\
\hline & & & A.M. & P.M. & A.M. & P.M. & A.M. & P.M. & A.M. & P.M. \\
\hline 22 & House \#4140 on Chavis Road-Front yard & 49.3 & 45 & 45 & 45 & 45 & 48 & 48 & 48 & 48 \\
\hline 23 & Waters-Large Cemetery-Entrance & 37.6 & 45 & 45 & 46 & 46 & 46 & 46 & 47 & 47 \\
\hline 24 & House \#227 Teaberry Lane & 42.0 & 40 & 40 & 42 & 42 & 42 & 42 & 43 & 43 \\
\hline 25 & $\begin{array}{l}\text { House \#5310 on Otto Williams Road-Back } \\
\text { yard }\end{array}$ & 52.4 & 36 & 37 & 37 & 38 & 38 & 38 & 39 & 39 \\
\hline 26 & House \#327 on Costner Road-Back yard & 44.4 & 35 & 36 & 36 & 36 & 37 & 38 & 38 & 38 \\
\hline 27 & House $\# 430$ Appletree Lane-Front yard & 40.3 & 29 & 29 & 30 & 30 & 31 & 31 & 32 & 32 \\
\hline 28 & $\begin{array}{l}\text { House on Sunshine Trail in Cobbly } \\
\text { Knob-Balcony }\end{array}$ & 41.8 & 33 & 33 & 33 & 34 & 35 & 35 & 36 & 36 \\
\hline 29 & Rental House on McKinzi Way-Front yard & 39.7 & 43 & 43 & 43 & 43 & 45 & 45 & 45 & 45 \\
\hline 30 & House on Shultz Road-Driveway & 39.6 & 43 & 43 & 43 & 43 & 45 & 45 & 45 & 45 \\
\hline 31 & Copeland Rental Cabins-Copeland Road & 47.1 & 43 & 43 & 43 & 43 & 45 & 45 & 45 & 45 \\
\hline 32 & House on The Way (last house)-Front yard & 41.1 & 43 & 43 & 44 & 44 & 45 & 45 & 46 & 46 \\
\hline 33 & $\begin{array}{l}\text { House on The Way (second to last } \\
\text { house)-Front deck }\end{array}$ & 45.6 & 41 & 41 & 42 & 43 & 42 & 43 & 44 & 44 \\
\hline 34 & House $\# 429$ on Appletree Lane & 50.8 & 46 & 46 & 46 & 46 & 49 & 49 & 49 & 49 \\
\hline 35 & $\begin{array}{l}\text { House at Intersection of Waters Cemetery } \\
\text { Road and Chavis Road }\end{array}$ & 49.5 & 39 & 39 & 39 & 40 & 42 & 42 & 42 & 42 \\
\hline 36 & House Off of Shultz Road & 46.9 & 45 & 45 & 47 & 47 & 47 & 47 & 48 & 48 \\
\hline 37 & $\begin{array}{l}\text { House \#916 Scenic Trail (Whistler's Watch) } \\
\text { in Cobbly Knob-Driveway }\end{array}$ & 41.3 & 41 & 41 & 41 & 41 & 43 & 43 & 43 & 43 \\
\hline 38 & $\begin{array}{l}\text { House on Sutton Place (Hidden Haven) in } \\
\text { Cobbly Knob-Driveway }\end{array}$ & 35.1 & 32 & 33 & 33 & 33 & 35 & 35 & 35 & 35 \\
\hline 39 & $\begin{array}{l}\text { Gateway Near House on Black Gum Gap } \\
\text { Road in Cobbly Knob }\end{array}$ & .54 .4 & 42 & 42 & 42 & 42 & 44 & 44 & 44 & 44 \\
\hline 40 & $\begin{array}{l}\text { House (under construction) at the End of } \\
\text { Squirrel Trail in Cobbly Knob-Near } \\
\text { driveway }\end{array}$ & 36.6 & 33 & 33 & 33 & 34 & 35 & 35 & 35 & 36 \\
\hline 41 & $\begin{array}{l}\text { House (uninhabited or under construction) on } \\
\text { Rocky Flatts Road }\end{array}$ & 48.4 & 44 & 44 & 44 & 44 & 46 & 46 & 46 & 46 \\
\hline
\end{tabular}


Table L.4. Noise level projections-interchange at Highway 416

(both options)-8B opened prior to $8 \mathrm{C}$ completion

\begin{tabular}{|c|c|c|c|c|c|c|c|c|c|c|}
\hline \multirow{4}{*}{$\begin{array}{l}\text { Site } \\
\text { no. }\end{array}$} & \multirow[b]{4}{*}{ Location } & \multicolumn{9}{|c|}{ Noise level (Leq) } \\
\hline & & \multirow[b]{3}{*}{ Existing } & \multicolumn{4}{|c|}{2006} & \multicolumn{4}{|c|}{2026} \\
\hline & & & \multicolumn{2}{|c|}{ Weekday } & \multicolumn{2}{|c|}{ Weekend } & \multicolumn{2}{|c|}{ Weekday } & \multicolumn{2}{|c|}{ Weekend } \\
\hline & & & A.M. & P.M. & A.M. & P.M. & A.M. & P.M. & A.M. & P.M. \\
\hline 1 & $\begin{array}{l}\text { Foothills Pkwy Sec. } 8 A-1 \text { st Scenic } \\
\text { Overlook from U.S. } 321\end{array}$ & 47.3 & 59 & 58 & 61 & 59 & 61 & 59 & 62 & 61 \\
\hline 2 & $\begin{array}{l}\text { Foothills Pkwy Sec. } 8 \mathrm{~A}-1 \text { st Scenic } \\
\text { Overlook from I-40 }\end{array}$ & 47.3 & 59 & 58 & 61 & 59 & 61 & 59 & 62 & 61 \\
\hline 3 & $\begin{array}{l}\text { Burnett Memorial Methodist } \\
\text { Church-Pittman Center }\end{array}$ & 49.0 & 43 & 43 & 43 & 43 & 45 & 45 & 45 & 45 \\
\hline 4 & $\begin{array}{l}\text { Pittman Center Elementary } \\
\text { School-Playground }\end{array}$ & 42.6 & 43 & 43 & 43 & 43 & 45 & 45 & 45 & 45 \\
\hline 5 & Pittman Center City Hall-Parking lot & 51.3 & 47 & 46 & 47 & 47 & 48 & 48 & 49 & 49 \\
\hline 6 & $\begin{array}{l}\text { House } \# 3355 \text { between Mile Markers } 20 \text { and } \\
21 \text { on U.S. } 321 \text {-Front yard. }\end{array}$ & 67.2 & 71 & 72 & 73 & 73 & 73 & 73 & 74 & 74 \\
\hline 7 & House $\# 5106$ on SR 416-Front yard & 47.7 & 43 & 43 & 43 & 43 & 45 & 45 & 45 & 45 . \\
\hline 8 & $\begin{array}{l}\text { House } \# 3010 \text { on Webb Creek Road-Front } \\
\text { yard }\end{array}$ & 40.7 & 43 & 43 & 43 & 43 & 45 & 45 & 45 & 45 \\
\hline 9 & House \#5005 on SR 416-Front yard & 62.4 & 43 & 43 & 43 & 43 & 45 & 45 & 45 & 45 \\
\hline 10 & House $\# 5215$ on SR 416-Front yard & 49.7 & 68 & 67 & 68 & 69 & 69 & 68 & 69 & 70 \\
\hline 11 & House \#5328 on SR 416-Front yard & 54.3 & 62 & 62 & 63 & 63 & 63 & 63 & 64 & 64 \\
\hline 12 & $\begin{array}{l}\text { Greenbrier View Rental Cottage on Tunis } \\
\text { Branch Road }\end{array}$ & 39.4 & 35 & 35 & 35 & 35 & 37 & 38 & 38 & 38 \\
\hline 13 & $\begin{array}{l}\text { House \#209 on Teaberry Hill Road-Front } \\
\text { yard }\end{array}$ & 39.1 & 45 & 45 & 45 & 45 & 47 & 47 & 47 & 47 \\
\hline 14 & $\begin{array}{l}\text { House \#4705 on Townsend Road—Front } \\
\text { yard }\end{array}$ & 45.8 & 41 & 41 & 42 & 42 & 42 & 42 & 43 & 43 \\
\hline 15 & $\begin{array}{l}\text { House at End of Branam Hollow } \\
\text { Road-Back yard of guest house }\end{array}$ & 38.7 & 38 & 38 & 38 & 38 & 40 & 40 & 40 & 40 \\
\hline 16 & $\begin{array}{l}\text { House \#612 on Branam Hollow } \\
\text { Road-Driveway }\end{array}$ & 35.5 & 30 & 31 & 31 & 31 & 32 & 32 & 33 & 33 \\
\hline 17 & $\begin{array}{l}\text { House \#5258 Off of Mathis Branch } \\
\text { Road-Front yard }\end{array}$ & 53.2 & 28 & 28 & 29 & 29 & 29 & 30 & 30 & 31 \\
\hline 18 & House Off of Rocky Flatts Road-Back yard & 64.0 & 39 & 39 & 40 & 40 & 41 & 41 & 42 & 42 \\
\hline 19 & House \#5039 on U.S. $321-$ Front yard & 63.1 & 69 & 70 & 71 & 71 & 71 & 71 & 72 & 72 \\
\hline 20 & House \#4022 on U.S. 321 -Back yard & 53.6 & 43 & 43 & 43 & 43 & 45 & 45 & 45 & 45 \\
\hline 21 & Young's Taxedermy Off U.S 321-Driveway & 51.8 & 59 & 59 & 60 & 60 & 60 & 60 & 61 & 61 \\
\hline
\end{tabular}


Table L.4. Continued

\begin{tabular}{|c|c|c|c|c|c|c|c|c|c|c|}
\hline \multirow{4}{*}{$\begin{array}{l}\text { Site } \\
\text { no. }\end{array}$} & \multirow{4}{*}{ Location } & \multicolumn{9}{|c|}{ Noise level (Leq) } \\
\hline & & \multirow[b]{3}{*}{ Existing } & \multicolumn{4}{|c|}{2006} & \multicolumn{4}{|c|}{2026} \\
\hline & & & \multicolumn{2}{|c|}{ Weekday } & \multicolumn{2}{|c|}{ Weekend } & \multicolumn{2}{|c|}{ Weekday } & \multicolumn{2}{|c|}{ Weekend } \\
\hline & & & A.M. & P.M. & A.M. & P.M. & A.M. & P.M. & A.M. & P.M. \\
\hline 22 & House $\# 4140$ on Chavis Road-Front yard & 49.3 & 45 & 45 & 45 & 45 & 48 & 48 & 48 & 48 \\
\hline 23 & Waters-Large Cemetery-Entrance & 37.6 & 45 & 45 & 46 & 46 & 46 & 46 & 47 & 47 \\
\hline 24 & House \#227 Teaberry Lane & 42.0 & 40 & 41 & 42 & 42 & 42 & 42 & 43 & 43 \\
\hline 25 & $\begin{array}{l}\text { House } \# 5310 \text { on Otto Williams Road-Back } \\
\text { yard }\end{array}$ & 52.4 & 36 & 37 & 37 & 38 & 38 & 38 & 39 & 39 \\
\hline 26 & House \#327 on Costner Road-Back yard & 44.4 & 35 & 36 & 36 & 36 & 37 & 38 & 38 & 38 \\
\hline 27 & House \#430 Appletree Lane-Front yard & 40.3 & 29 & 29 & 30 & 30 & 31 & 31 & 32 & 32 \\
\hline 28 & $\begin{array}{l}\text { House on Sunshine Trail in Cobbly } \\
\text { Knob-Balcony }\end{array}$ & 41.8 & 33 & 33 & 33 & 34 & 35 & 35 & 36 & 36 \\
\hline 29 & Rental House on McKinzi Way-Front yard & 39.7 & 43 & 43 & 43 & 43 & 45 & 45 & 45 & 45 \\
\hline 30 & House on Shultz Road-Driveway & 39.6 & 43 & 43 & 43 & 43 & 45 & 45 & 45 & 45 \\
\hline 31 & Copeland Rental Cabins-Copeland Road & 47.1 & 43 & 43 & 43 & 43 & 45 & 45 & 45 & 45 \\
\hline 32 & House on The Way (last house)-Front yard & 41.1 & 43 & 43 & 44 & 44 & 45 & 45 & 46 & 46 \\
\hline 33 & $\begin{array}{l}\text { House on The Way (second to last } \\
\text { house)-Front deck }\end{array}$ & 45.6 & 41 & 41 & 42 & 43 & 43 & 43 & 44 & 44 \\
\hline 34 & House \#429 on Appletree Lane & 50.8 & 46 & 46 & 46 & 46 & 49 & 49 & 49 & 49 \\
\hline 35 & $\begin{array}{l}\text { House at Intersection of Waters Cemetery } \\
\text { Road and Chavis Road }\end{array}$ & 49.5 & 39 & 39 & 39 & 40 & 42 & 42 & 42 & 42 \\
\hline 36 & House Off of Shultz Road & 46.9 & 45 & 46 & 47 & 47 & 47 & 47 & 48 & 48 \\
\hline 37 & $\begin{array}{l}\text { House \#916 Scenic Trail (Whistler's Watch) } \\
\text { in Cobbly Knob-Driveway }\end{array}$ & 41.3 & 41 & 41 & 41 & 41 & 43 & 43 & 43 & 43 \\
\hline 38 & $\begin{array}{l}\text { House on Sutton Place (Hidden Haven) in } \\
\text { Cobbly Knob-Driveway }\end{array}$ & 35.1 & 32 & 33 & 33 & 33 & 35 & 35 & 35 & 35 \\
\hline 39 & $\begin{array}{l}\text { Gateway Near House on Black Gum Gap } \\
\text { Road in Cobbly Knob }\end{array}$ & 54.4 & 42 & 42 & 42 & 42 & 44 & 44 & 44 & 44 \\
\hline 40 & $\begin{array}{l}\text { House (under construction) at the End of } \\
\text { Squirrel Trail in Cobbly Knob-Near } \\
\text { driveway }\end{array}$ & 36.6 & 33 & 33 & 33 & 34 & 35 & 35 & 35 & 36 \\
\hline 41 & $\begin{array}{l}\text { House (uninhabited or under construction) on } \\
\text { Rocky Flatts Road }\end{array}$ & 48.4 & 44 & 44 & 44 & 44 & 46 & 46 & 46 & 46 \\
\hline
\end{tabular}


Table L.5. Noise level projections-interchange at U.S. 321

(both options) -8B opened prior to $8 \mathrm{C}$ completion

\begin{tabular}{|c|c|c|c|c|c|c|c|c|c|c|}
\hline \multirow{4}{*}{$\begin{array}{l}\text { Site } \\
\text { no. }\end{array}$} & \multirow[b]{4}{*}{ Location } & \multicolumn{9}{|c|}{ Noise level (Leq) } \\
\hline & & \multirow[b]{3}{*}{ Existing } & \multicolumn{4}{|c|}{2006} & \multicolumn{4}{|c|}{2026} \\
\hline & & & \multicolumn{2}{|c|}{ Weekday } & \multicolumn{2}{|c|}{ Weekend } & \multicolumn{2}{|c|}{ Weekday } & \multicolumn{2}{|c|}{ Weekend } \\
\hline & & & A.M. & P.M. & A.M. & P.M. & A.M. & P.M. & A.M. & P.M. \\
\hline 1 & $\begin{array}{l}\text { Foothills Pkwy Sec. } 8 \text { A-1st Scenic } \\
\text { Overlook from U.S. } 321\end{array}$ & 47.3 & 59 & 58 & 61 & 59 & 61 & 59 & 62 & 61 \\
\hline 2 & $\begin{array}{l}\text { Foothills Pkwy Sec. } 8 A-1 \text { st Scenic } \\
\text { Overlook from } I-40\end{array}$ & 47.3 & 59 & 58 & 61 & 59 & 61 & 59 & 62 & 61 \\
\hline 3 & $\begin{array}{l}\text { Burnett Memorial Methodist } \\
\text { Church-Pittman Center }\end{array}$ & 49.0 & 43 & 43 & 43 & 43 & 45 & 45 & 45 & 45 \\
\hline 4 & $\begin{array}{l}\text { Pittman Center Elementary } \\
\text { School-Playground }\end{array}$ & 42.6 & 43 & 43 & 43. & 43 & 45 & 45 & 45 & 45 \\
\hline 5 & Pittman Center City Hall-Parking lot & 51.3 & 45 & 45 & 46 & 46 & 47 & 47 & 47 & 48 \\
\hline 6 & $\begin{array}{l}\text { House \#3355 between Mile Markers } 20 \text { and } \\
21 \text { on U.S. } 321 \text {-Front yard }\end{array}$ & 67.2 & 72 & 72 & 73 & 73 & 73 & 73 & 74 & 75 \\
\hline 7 & House \#5106 on SR 416-Front yard & 47.7 & 43 & 43 & 43 & 43 & 45 & 45 & 45 & 45 \\
\hline 8 & $\begin{array}{l}\text { House \#3010 on Webb Creek Road-Front } \\
\text { yard }\end{array}$ & 40.7 & 43 & 43 & 43 & 43 & 45 & .45 & 45 & 45 \\
\hline 9 & House \#5005 on SR 416-Front yard & 62.4 & 43 & 43 & 43 & 43 & 45 & 45 & 45 & 45 \\
\hline 10 & House \#5215 on SR 416-Front yard & 49.7 & 65 & 65 & 66 & 66 & 66 & 66 & 66 & 67 \\
\hline 11 & House \#5328 on SR 416-Front yard & 54.3 & 60 & 60 & 60 & 61 & 60 & 60 & 60 & 62 \\
\hline 12 & $\begin{array}{l}\text { Greenbrier View Rental Cottage on Tunis } \\
\text { Branch Road }\end{array}$ & 39.4 & 35 & 35 & 35 & 35 & 37 & 38 & 38 & 38 \\
\hline 13 & $\begin{array}{l}\text { House \#209 on Teaberry Hill Road-Front } \\
\text { yard }\end{array}$ & 39.1 & 45 & 45 & 46 & 46 & 47 & 47 & 48 & 48 \\
\hline 14 & $\begin{array}{l}\text { House \#4705 on Townsend Road-Front } \\
\text { yard }\end{array}$ & 45.8 & 41 & 41 & 42 & 42 & 42 & 42 & 44 & 44 \\
\hline 15 & $\begin{array}{l}\text { House at End of Branam Hollow } \\
\text { Road-Back yard of guest house }\end{array}$ & 38.7 & 38 & 38 & 38 & 38 & 40 & 40 & 40 & 40 \\
\hline 16 & $\begin{array}{l}\text { House \#612 on Branam Hollow } \\
\text { Road-Driveway }\end{array}$ & 35.5 & 30 & 31 & 31 & 31 & 32 & 32 & 33 & 33 \\
\hline 17 & $\begin{array}{l}\text { House \#5258 Off of Mathis Branch } \\
\text { Road-Front yard }\end{array}$ & 53.2 & 28 & 28 & 29 & 29 & 29 & 30 & 30 & 31 \\
\hline 18 & House Off of Rocky Flatts Road-Back yard & 64.0 & 39 & 39 & 40 & 40 & 41 & 41 & 42 & 42 \\
\hline 19 & House \#5039 on U.S. 321 -Front yard & 63.1 & 70 & 70 & 71 & 71 & 71 & 71 & 72 & 73 \\
\hline 20 & House \#4022 on U.S. 321 -Back yard & 53.6 & 43 & 43 & 43 & 43 & 45 & 45 & 45 & 45 \\
\hline 21 & Young's Taxedermy Off U.S 321-Driveway & 51.8 & 59 & 59 & 60 & 60 & 60 & 60 & 62 & 62 \\
\hline
\end{tabular}


Table L.5. Continued

\begin{tabular}{|c|c|c|c|c|c|c|c|c|c|c|}
\hline \multirow{4}{*}{$\begin{array}{l}\text { Site } \\
\text { no. }\end{array}$} & \multirow[b]{4}{*}{ Location } & \multicolumn{9}{|c|}{ Noise level (Leq) } \\
\hline & & \multirow[b]{3}{*}{ Existing } & \multicolumn{4}{|c|}{2006} & \multicolumn{4}{|c|}{2026} \\
\hline & & & \multicolumn{2}{|c|}{ Weekday } & \multicolumn{2}{|c|}{ Weekend } & \multicolumn{2}{|c|}{ Weekday } & \multicolumn{2}{|c|}{ Weekend } \\
\hline & & & A.M. & P.M. & A.M. & P.M. & A.M. & P.M. & A.M. & P.M. \\
\hline 22 & House $\# 4140$ on Chavis Road-Front yard & 49.3 & 45 & 45 & 45 & 45 & 48 & 48 & 48 & 48 \\
\hline 23 & Waters-Large Cemetery-Entrance & 37.6 & 45 & 45 & 46 & 46 & 46 & 46 & 47 & 47 \\
\hline 24 & House \#227 Teaberry Lane & 42.0 & 41 & 41 & 42 & 42 & 42 & 42 & 43 & 43 \\
\hline 25 & $\begin{array}{l}\text { House } \# 5310 \text { on Otto Williams Road-Back } \\
\text { yard }\end{array}$ & 52.4 & 36 & 37 & 37 & 38 & 38 & 38 & 39 & 39 \\
\hline 26 & House \#327 on Costner Road-Back yard & 44.4 & 35 & 36 & 36 & 36 & 37 & 38 & 38 & 38 \\
\hline 27 & House \#430 Appletree Lane-Front yard & 40.3 & 29 & 29 & 30 & 30 & 31 & 31 & 32 & 32 \\
\hline 28 & $\begin{array}{l}\text { House on Sunshine Trail in Cobbly } \\
\text { Knob-Balcony }\end{array}$ & 41.8 & 33 & 33 & 33 & 34 & 35 & 35 & 36 & 36 \\
\hline 29 & Rental House on McKinzi Way - Front yard & 39.7 & 43 & 43 & 43 & 43 & 45 & 45 & 45 & 45 \\
\hline 30 & House on Shultz Road-Driveway & 39.6 & 43 & 43 & 43 & 43 & 45 & 45 & 45 & 45 \\
\hline 31 & Copeland Rental Cabins-Copeland Road & 47.1 & 43 & 43 & 43 & 43 & 45 & 45 & 45 & 45 \\
\hline 32 & House on The Way (last house)-Front yard & 41.1 & 43 & 43 & 44 & 44 & 45 & 45 & 46 & 46 \\
\hline 33 & $\begin{array}{l}\text { House on The Way (second to last } \\
\text { house)-Front deck }\end{array}$ & 45.6 & 42 & 42 & 43 & 43 & 43 & 43 & 44 & 44 \\
\hline 34 & House $\# 429$ on Appletree Lane & 50.8 & 46 & 46 & 46 & 46 & 49 & 49 & 49 & 49 \\
\hline 35 & $\begin{array}{l}\text { House at Intersection of Waters Cemetery } \\
\text { Road and Chavis Road }\end{array}$ & 49.5 & 39 & 39 & 39 & 40 & 42 & 42 & 42 & 42 \\
\hline 36 & House Off of Shultz Road & 46.9 & 46 & 46 & 47 & 47 & 47 & 47 & 48 & 49 \\
\hline 37 & $\begin{array}{l}\text { House \#916 Scenic Trail (Whistler's Watch) } \\
\text { in Cobbly Knob-Driveway }\end{array}$ & 41.3 & 41 & 41 & 41 & 41 & 43 & 43 & 43 & 43 \\
\hline 38 & $\begin{array}{l}\text { House on Sutton Place (Hidden Haven) in } \\
\text { Cobbly Knob-Driveway }\end{array}$ & 35.1 & 32 & 33 & 33 & 33 & 35 & 35 & 35 & 35 \\
\hline 39 & $\begin{array}{l}\text { Gateway Near House on Black Gum Gap } \\
\text { Road in Cobbly Knob }\end{array}$ & 54.4 & 42 & 42 & 42 & 42 & 44 & 44 & 44 & 44 \\
\hline 40 & $\begin{array}{l}\text { House (under construction) at the End of } \\
\text { Squirrel Trail in Cobbly Knob-Near } \\
\text { driveway }\end{array}$ & 36.6 & 33 & 33 & 33 & 34 & 35 & 35 & 35 & 36 \\
\hline 41 & $\begin{array}{l}\text { House (uninhabited or under construction) on } \\
\text { Rocky Flatts Road }\end{array}$ & 48.4 & 44 & 44 & 44 & 44 & 46 & 46 & 46 & 46 \\
\hline
\end{tabular}


Table L.6. Noise level projections-no-build alternative

\begin{tabular}{|c|c|c|c|c|c|c|c|c|c|c|}
\hline \multirow{4}{*}{$\begin{array}{l}\text { Site } \\
\text { no. }\end{array}$} & \multirow[b]{4}{*}{ Location } & \multicolumn{9}{|c|}{ Noise level (Leq) } \\
\hline & & \multirow[b]{3}{*}{ Existing } & \multicolumn{4}{|c|}{2006} & \multicolumn{4}{|c|}{2026} \\
\hline & & & \multicolumn{2}{|c|}{ Weekday } & \multicolumn{2}{|c|}{ Weekend } & \multicolumn{2}{|c|}{ Weekday } & \multicolumn{2}{|c|}{ Weekend } \\
\hline & & & A.M. & P.M. & A.M. & P.M. & A.M. & P.M. & A.M. & P.M. \\
\hline 1 & $\begin{array}{l}\text { Foothills Pkwy Sec. } 8 A-1 \text { st Scenic } \\
\text { Overlook from U.S. } 321\end{array}$ & 47.3 & 59 & 57 & 60 & 58 & 60 & 58 & 61 & 59 \\
\hline 2 & $\begin{array}{l}\text { Foothills Pkwy Sec. } 8 A-1 \text { st Scenic } \\
\text { Overlook from } 1-40\end{array}$ & 47.3 & 59 & 57 & 60 & 58 & 60 & 58 & 61 & 59 \\
\hline 3 & $\begin{array}{l}\text { Burnett Memorial Methodist } \\
\text { Church-Pittman Center }\end{array}$ & 49.0 & 42 & 42 & 42 & 42 & 45 & 45 & 45 & 45 \\
\hline 4 & $\begin{array}{l}\text { Pittman Center Elementary } \\
\text { School-Playground }\end{array}$ & 42.6 & 42 & 42 & 42 & 42 & 45 & 45 & 45 & 45 \\
\hline 5 & Pittman Center City Hall-Parking lot & 51.3 & 45 & 45 & 45 & 46 & 46 & 47 & 47 & 47 \\
\hline 6 & $\begin{array}{l}\text { House \#3355 between Mile Markers } 20 \text { and } \\
21 \text { on U.S. } 321 \text {-Front yard }\end{array}$ & 67.2 & 72 & 72 & 73 & 73 & 72 & 73 & 75 & 75 \\
\hline 7 & House \#5106 on SR 416-Front yard & 47.7 & 42 & 42 & 42 & 42 & 45 & 45 & 45 & 45 \\
\hline 8 & $\begin{array}{l}\text { House \#3010 on Webb Creek Road-Front } \\
\text { yard }\end{array}$ & 40.7 & 42 & 42 & 42 & 42 & 45 & 45 & 45 & 45 \\
\hline 9 & House $\# 5005$ on SR $416-$ Front yard & 62.4 & 42 & 42 & 42 & 42 & 45 & 45 & 45 & 45 \\
\hline 10 & House \#5215 on SR 416-Front yard & 49.7 & 65 & 65 & 66 & 66 & 65 & 66 & 66 & 67 \\
\hline 11 & House \#5328 on SR 416-Front yard & 54.3 & 60 & 60 & 60 & 61 & 59 & 60 & 60 & 62 \\
\hline 12 & $\begin{array}{l}\text { Greenbrier View Rental Cottage on Tunis } \\
\text { Branch Road }\end{array}$ & 39.4 & 34 & 34 & 34 & 34 & 37 & 37 & 37 & 37 \\
\hline 13 & $\begin{array}{l}\text { House \#209 on Teaberry Hill Road-Front } \\
\text { yard }\end{array}$ & 39.1 & 45 & 45 & 45 & 46 & 47 & 47 & 47 & 47 \\
\hline 14 & $\begin{array}{l}\text { House \#4705 on Townsend Road-Front } \\
\text { yard }\end{array}$ & 45.8 & 41 & 41 & 42 & 42 & 41 & 42 & 43 & 43 \\
\hline 15 & $\begin{array}{l}\text { House at End of Branam Hollow } \\
\text { Road-Back yard of guest house }\end{array}$ & 38.7 & 37 & 37 & 37 & 37 & 40 & 40 & 40 & 40 \\
\hline 16 & $\begin{array}{l}\text { House \#612 on Branam Hollow } \\
\text { Road-Driveway }\end{array}$ & 35.5 & 26 & 26 & 26 & 26 & 29 & 29 & 29 & 29 \\
\hline 17 & $\begin{array}{l}\text { House \#5258 Off of Mathis Branch } \\
\text { Road-Front yard }\end{array}$ & 53.2 & 19 & 19 & 19 & 19 & 22 & 22 & 22 & 22 \\
\hline 18 & House Off of Rocky Flatts Road-Back yard & 64.0 & 34 & 34 & 34 & 34 & 36 & 36 & 36 & 36 \\
\hline 19 & House \#5039 on U.S. 321 - Front yard & 63.1 & 70 & 70 & 71 & 71 & 70 & 71 & 72 & 73 \\
\hline 20 & House \#4022 on U.S. $321-$ Back yard & 53.6 & 42 & 42 & 42 & 42 & 45 & 45 & 45 & 45 \\
\hline 21 & Young's Taxedermy Off U.S 321-Driveway & 51.8 & 59 & 59 & 60 & 61 & 59 & 60 & 62 & 62 \\
\hline 22 & House \#4140 on Chavis Road-Front yard & 49.3 & 45 & 45 & 45 & 45 & 48 & 48 & 48 & 48 \\
\hline
\end{tabular}


Table L.6. Continued

\begin{tabular}{|c|c|c|c|c|c|c|c|c|c|c|}
\hline \multirow{4}{*}{$\begin{array}{l}\text { Site } \\
\text { no. }\end{array}$} & \multirow[b]{4}{*}{ Location } & \multicolumn{9}{|c|}{ Noise level (Leq) } \\
\hline & & \multirow[b]{3}{*}{ Existing } & \multicolumn{4}{|c|}{2006} & \multicolumn{4}{|c|}{2026} \\
\hline & & & \multicolumn{2}{|c|}{ Weekday } & \multicolumn{2}{|c|}{ Weekend } & \multicolumn{2}{|c|}{ Weekday } & \multicolumn{2}{|c|}{ Weekend } \\
\hline & & & A.M. & P.M. & A.M. & P.M. & A.M. & P.M. & A.M. & P.M. \\
\hline 23 & Waters-Large Cemetery-Entrance & 37.6 & 45 & 45 & 46 & 46 & 46 & 46 & 48 & 47 \\
\hline 24 & House \#227 Teaberry Lane & 42.0 & 40 & 41 & 42 & 42 & 41 & 42 & 43 & 43 \\
\hline 25 & $\begin{array}{l}\text { House } \# 5310 \text { on Otto Williams Road-Back } \\
\text { yard }\end{array}$ & 52.4 & 26 & 26 & 26 & 26 & 29 & 29 & 29 & 29 \\
\hline 26 & House $\# 327$ on Costner Road-Back yard & 44.4 & 32 & 32 & 32 & 32 & 34 & 34 & 34 & 34 \\
\hline 27 & House $\# 430$ Appletree Lane-Front yard & 40.3 & 24 & 24 & 24 & 24 & 26 & 26 & 26 & 26 \\
\hline 28 & $\begin{array}{l}\text { House on Sunshine Trail in Cobbly } \\
\text { Knob-Balcony }\end{array}$ & 41.8 & 31 & 31 & 31 & 31 & 33 & 33 & 33 & 33 \\
\hline 29 & Rental House on McKinzi Way-Front yard & 39.7 & 42 & 42 & 42 & 42 & 45 & 45 & 45 & 45 \\
\hline 30 & House on Shultz Road-Driveway & 39.6 & 42 & 42 & 42 & 42 & 45 & 45 & 45 & 45 \\
\hline 31 & Copeland Rental Cabins-Copeland Road & 47.1 & 42 & 42 & 42 & 42 & 45 & 45 & 45 & 45 \\
\hline 32 & House on The Way (last house)-Front yard & 41.1 & 43 & 43 & 44 & 44 & 45 & 45 & 46 & 46 \\
\hline 33 & $\begin{array}{l}\text { House on The Way (second to last } \\
\text { house)-Front deck }\end{array}$ & 45.6 & 41 & 42 & 43 & 43 & 42 & 43 & 44 & 44 \\
\hline 34 & House $\# 429$ on Appletree Lane & 50.8 & 46 & 46 & 46 & 46 & 49 & 49 & 49 & 49 \\
\hline 35 & $\begin{array}{l}\text { House at Intersection of Waters Cemetery } \\
\text { Road and Chavis Road }\end{array}$ & 49.5 & 39 & 39 & 39 & 39 & 42 & 42 & 42 & 42 \\
\hline 36 & House Off of Shultz Road & 46.9 & 46 & 46 & 47 & 47 & 46 & 47 & 48 & 48 \\
\hline 37 & $\begin{array}{l}\text { House \#916 Scenic Trail (Whistler's Watch) } \\
\text { in Cobbly Knob-Driveway }\end{array}$ & 41.3 & 40 & 40 & 40 & 40 & 43 & 43 & 43 & 43 \\
\hline 38 & $\begin{array}{l}\text { House on Sutton Place (Hidden Haven) in } \\
\text { Cobbly Knob-Driveway }\end{array}$ & 35.1 & 30 & 30 & 30 & 30 & 33 & 33 & 33 & 33 \\
\hline 39 & $\begin{array}{l}\text { Gateway Near House on Black Gum Gap } \\
\text { Road in Cobbly Knob }\end{array}$ & 54.4 & 42 & 42 & 42 & 42 & 44 & 44 & 44 & 44 \\
\hline 40 & $\begin{array}{l}\text { House (under construction) at the End of } \\
\text { Squirrel Trail in Cobbly Knob-Near } \\
\text { driveway }\end{array}$ & 36.6 & 30 & 30 & 30 & 30 & 33 & 33 & 33 & 33 \\
\hline 41 & $\begin{array}{l}\text { House (uninhabited or under construction) on } \\
\text { Rocky Flatts Road }\end{array}$ & 48.4 & 41 & 41 & 41 & 41 & 44 & 44 & 44 & 44 \\
\hline
\end{tabular}


Table L.7. Noise level comparison-no-build vs construct $8 \mathrm{~B}$ with no interchanges

\begin{tabular}{|c|c|c|c|c|c|c|c|c|c|c|}
\hline \multirow{4}{*}{$\begin{array}{l}\text { Site } \\
\text { no. }\end{array}$} & \multirow[b]{4}{*}{ Location } & \multicolumn{9}{|c|}{ Noise level (Leq) } \\
\hline & & \multirow[b]{3}{*}{ Existing } & \multicolumn{4}{|c|}{2006} & \multicolumn{4}{|c|}{2026} \\
\hline & & & \multicolumn{2}{|c|}{ Weekday } & \multicolumn{2}{|c|}{ Weekend } & \multicolumn{2}{|c|}{ Weekday } & \multicolumn{2}{|c|}{ Weekend } \\
\hline & & & A.M. & P.M. & A.M. & P.M. & A.M. & P.M. & A.M. & P.M. \\
\hline 1 & $\begin{array}{l}\text { Foothills Pkwy Sec. } 8 \text { A-1st Scenic } \\
\text { Overlook from U.S. } 321\end{array}$ & 47.3 & 0 & 1 & 1 & 1 & 1 & 1 & 1 & 2 \\
\hline 2 & $\begin{array}{l}\text { Foothills Pkwy Sec. 8A-1st Scenic } \\
\text { Overlook from I-40 }\end{array}$ & 47.3 & 0 & 1 & 1 & 1 & 1 & 1 & 1 & 2 \\
\hline 3 & $\begin{array}{l}\text { Burnett Memorial Methodist } \\
\text { Church-Pittman Center }\end{array}$ & 49.0 & 1 & 1 & 1 & 1 & 0 & 0 & 0 & 0 \\
\hline 4 & $\begin{array}{l}\text { Pittman Center Elementary } \\
\text { School-Playground }\end{array}$ & 42.6 & 1 & 1 & 1 & 1 & 0 & 0 & 0 & 0 \\
\hline 5 & Pittman Center City Hall-Parking lot & 51.3 & 0 & 0 & 1 & 0 & 1 & 0 & $\mathbf{0}$ & 1 \\
\hline 6 & $\begin{array}{l}\text { House \#3355 between Mile Markers } 20 \text { and } \\
21 \text { on U.S. } 321 \text {-Front yard }\end{array}$ & 67.2 & -1 & -1 & 0 & 0 & 1 & 0 & -1 & -1 \\
\hline 7 & House \#5106 on SR 416-Front yard & 47.7 & 1 & 1 & 1 & 1 & 0 & 0 & 0 & 0 \\
\hline 8 & $\begin{array}{l}\text { House \#3010 on Webb Creek Road-Front } \\
\text { yard }\end{array}$ & 40.7 & 1 & 1 & 1 & 1 & 0 & 0 & 0 & 0 \\
\hline 9 & House $\# 5005$ on SR 416-Front yard & 62.4 & 1 & 1 & 1 & 1 & 0 & 0 & 0 & 0 \\
\hline 10 & House $\# 5215$ on SR 416-Front yard & 49.7 & 0 & $\mathbf{0}$ & 0 & 0 & 1 & 0 & 0 & 0 \\
\hline 11 & House \#5328 on SR 416-Front yard & 54.3 & 0 & 0 & 0 & 0 & 1 & 0 & 0 & 0 \\
\hline 12 & $\begin{array}{l}\text { Greenbrier View Rental Cottage on Tunis } \\
\text { Branch Road }\end{array}$ & 39.4 & 1 & 1 & 1 & 1 & 0 & 1 & 1 & 1 \\
\hline 13 & $\begin{array}{l}\text { House \#209 on Teaberry Hill Road-Front } \\
\text { yard }\end{array}$ & 39.1 & 0 & $\mathbf{0}$ & 0 & -1 & 0 & 0 & 0 & 0 \\
\hline 14 & $\begin{array}{l}\text { House \#4705 on Townsend Road-Front } \\
\text { yard }\end{array}$ & 45.8 & -1 & 0 & 0 & $\mathbf{0}$ & 1 & 0 & 0 & 0 \\
\hline 15 & $\begin{array}{l}\text { House at End of Branam Hollow } \\
\text { Road-Back yard of guest house }\end{array}$ & 38.7 & 1 & 1 & 1 & 1 & $\mathbf{0}$ & 0 & 0 & 0 \\
\hline 16 & $\begin{array}{l}\text { House } \# 612 \text { on Branam Hollow } \\
\text { Road-Driveway }\end{array}$ & 35.5 & 4 & 5 & 5 & 5 & 3 & 3 & 4 & 4 \\
\hline 17 & $\begin{array}{l}\text { House \#5258 Off of Mathis Branch } \\
\text { Road-Front yard }\end{array}$ & 53.2 & 9 & 9 & 10 & 10 & 7 & 8 & 8 & 9 \\
\hline 18 & House Off of Rocky Flatts Road-Back yard & 64.0 & 5 & 5 & 6 & 6 & 5 & 5 & 6 & 6 \\
\hline 19 & House \#5039 on U.S. 321 -Front yard & 63.1 & -1 & -1 & 0 & 0 & 1 & 0 & 0 & -1 \\
\hline 20 & House $\# 4022$ on U.S. $321-$ Back yard & 53.6 & 1 & 1 & 1 & 1 & 0 & 0 & 0 & 0 \\
\hline 21 & Young's Taxedermy Off U.S 321-Driveway & 51.8 & -1 & 0 & 0 & -1 & 1 & 0 & -1 & -1 \\
\hline 22 & House \#4140 on Chavis Road-Front yard & 49.3 & 0 & 0 & 0 & 0 & 0 & 0 & 0 & 0 \\
\hline
\end{tabular}


Table L.7. Continued

\begin{tabular}{|c|c|c|c|c|c|c|c|c|c|c|}
\hline \multirow{4}{*}{$\begin{array}{l}\text { Site } \\
\text { no. }\end{array}$} & \multirow[b]{4}{*}{ Location } & \multicolumn{9}{|c|}{ Noise level (Leq) } \\
\hline & & \multirow[b]{3}{*}{ Existing } & \multicolumn{4}{|c|}{2006} & \multicolumn{4}{|c|}{2026} \\
\hline & & & \multicolumn{2}{|c|}{ Weekday } & \multicolumn{2}{|c|}{ Weekend } & \multicolumn{2}{|c|}{ Weekday } & \multicolumn{2}{|c|}{ Weekend } \\
\hline & & & A.M. & P.M. & A.M. & P.M. & A.M. & P.M. & A.M. & P.M. \\
\hline 23 & Waters-Large Cemetery-Entrance & 37.6 & 0 & 0 & $\mathbf{0}$ & 0 & 0 & 0 & -1 & 0 \\
\hline 24 & House \#227 Teaberry Lane & 42.0 & 0 & -1 & 0 & 0 & 1 & 0 & 0 & 0 \\
\hline 25 & $\begin{array}{l}\text { House \#5310 on Otto Williams Road-Back } \\
\text { yard }\end{array}$ & 52.4 & 10 & 11 & 11 & 12 & 9 & 9 & 10 & 10 \\
\hline 26 & House \#327 on Costner Road-Back yard & 44.4 & 3 & 4 & 4 & 4 & 3 & 4 & 4 & 4 \\
\hline 27 & House \#430 Appletree Lăne-Front yard & 40.3 & 5 & 5 & 6 & 6 & 5 & 5 & 6 & 6 \\
\hline 28 & $\begin{array}{l}\text { House on Sunshine Trail in Cobbly } \\
\text { Knob-Balcony }\end{array}$ & 41.8 & 2 & 2 & 2 & 3 & 2 & 2 & 3 & 3 \\
\hline 29 & Rental House on McKinzi Way-Front yard & 39.7 & $\cdot 1$ & 1 & 1 & 1 & 0 & 0 & 0 & 0 \\
\hline 30 & House on Shultz Road-Driveway & 39.6 & 1 & 1 & 1 & 1 & 0 & 0 & 0 & 0 \\
\hline 31 & Copeland Rental Cabins-Copeland Road & 47.1 & 1 & 1 & 1 & 1 & 0 & 0 & 0 & 0 \\
\hline 32 & House on The Way (last house)-Front yard & 41.1 & 0 & 0 & 0 & 0 & 0 & 0 & 0 & 0 \\
\hline 33 & $\begin{array}{l}\text { House on The Way (second to last } \\
\text { house)-Front deck }\end{array}$ & 45.6 & 0 & -1 & -1 & 0 & 1 & 0 & 0 & 0 \\
\hline 34 & House $\# 429$ on Appletree Lane & 50.8 & 0 & 0 & 0 & 0 & 0 & 0 & 0 & 0 \\
\hline 35 & $\begin{array}{l}\text { House at Intersection of Waters Cemetery } \\
\text { Road and Chavis Road }\end{array}$ & 49.5 & 0 & 0 & 1 & 1 & 0 & 0 & 0 & 0 \\
\hline 36 & House Off of Shultz Road & 46.9 & -1 & -1 & $\mathbf{0}$ & 0 & 1 & 0 & 0 & 0 \\
\hline 37 & $\begin{array}{l}\text { House } \$ 916 \text { Scenic Trail (Whistler's Watch) } \\
\text { in Cobbly Knob-Driveway }\end{array}$ & 41.3 & 1 & 1 & 1 & 1 & 0 & 0 & 0 & 0 \\
\hline 38 & $\begin{array}{l}\text { House on Sutton Place (Hidden Haven) in } \\
\text { Cobbly Knob-Driveway }\end{array}$ & 35.1 & 2 & 3 & 3 & 3 & 2 & 2 & 2 & 2 \\
\hline 39 & $\begin{array}{l}\text { Gateway Near House on Black Gum Gap } \\
\text { Road in Cobbly Knob }\end{array}$ & 54.4 & 0 & 0 & 0 & 0 & 0 & 0 & 0 & 0 \\
\hline 40 & $\begin{array}{l}\text { House (under construction) at the End of } \\
\text { Squirrel Trail in Cobbly Knob-Near } \\
\text { driveway }\end{array}$ & 36.6 & 3 & 3 & 3 & 4 & 2 & 2 & 2 & 3 \\
\hline 41 & $\begin{array}{l}\text { House (uninhabited or under construction) on } \\
\text { Rocky Flatts Road }\end{array}$ & 48.4 & 3 & 3 & 3 & 3 & 2 & 2 & 2 & 2 \\
\hline
\end{tabular}


Table L.8. Comparison of no-build alternative to interchange at Highway 416 (both options)-8B not opened until 8C completion

\begin{tabular}{|c|c|c|c|c|c|c|c|c|c|c|}
\hline \multirow{4}{*}{$\begin{array}{l}\text { Site } \\
\text { no. }\end{array}$} & \multirow[b]{4}{*}{ Location } & \multicolumn{9}{|c|}{ Noise level (Leq) } \\
\hline & & \multirow[b]{3}{*}{ Existing } & \multicolumn{4}{|c|}{2006} & \multicolumn{4}{|c|}{2026} \\
\hline & & & \multicolumn{2}{|c|}{ Weekday } & \multicolumn{2}{|c|}{ Weekend } & \multicolumn{2}{|c|}{ Weekday } & \multicolumn{2}{|c|}{ Weekend } \\
\hline & & & A.M. & P.M. & A.M. & P.M. & A.M. & P.M. & A.M. & P.M. \\
\hline 1 & $\begin{array}{l}\text { Foothills Pkwy Sec. } 8 \mathrm{~A}-1 \text { st Scenic } \\
\text { Overlook from U.S. } 321\end{array}$ & 47.3 & 0 & 1 & 1 & 1 & 1 & 1 & 1 & 2 \\
\hline 2 & $\begin{array}{l}\text { Foothills Pkwy Sec. } 8 \mathrm{~A}-1 \text { st Scenic } \\
\text { Overlook from I-40 }\end{array}$ & 47.3 & 0 & 1 & 1 & 1 & 1 & 1 & 1 & 2 \\
\hline 3 & $\begin{array}{l}\text { Burnett Memorial Methodist } \\
\text { Church-Pittman Center }\end{array}$ & 49.0 & 1 & 1 & 1 & 1 & 0 & 0 & 0 & 0 \\
\hline 4 & $\begin{array}{l}\text { Pittman Center Elementary } \\
\text { School-Playground }\end{array}$ & 42.6 & 1 & 1 & 1 & 1 & 0 & 0 & 0 & 0 \\
\hline 5 & Pittman Center City Hall—Parking lot & 51.3 & 1 & 1 & 1 & 1 & 1 & 0 & 0 & 1 \\
\hline 6 & $\begin{array}{l}\text { House } \# 3355 \text { between Mile Markers } 20 \text { and } \\
21 \text { on U.S. } 321 \text {-Front yard }\end{array}$ & 67.2 & -1 & 0 & 0 & 0 & 1 & 0 & -1 & -1 \\
\hline 7 & House $\# 5106$ on SR 416-Front yard & 47.7 & 1 & 1 & 1 & 1 & 0 & 0 & 0 & 0 \\
\hline 8 & $\begin{array}{l}\text { House \#3010 on Webb Creek Road-Front } \\
\text { yard }\end{array}$ & 40.7 & 1 & 1 & 1 & 1 & 0 & 0 & 0 & 0 \\
\hline 9 & House \#5005 on SR 416-Front yard & 62.4 & 1 & 1 & 1 & 1 & 0 & 0 & 0 & 0 \\
\hline 10 & House \#5215 on SR 416-Front yard & 49.7 & 1 & 2 & 0 & 2 & 1 & 1 & 0 & 1 \\
\hline 11 & House \#5328 on SR 416-Front yard & 54.3 & 0 & 1 & 0 & 1 & 1 & 1 & 0 & 0 \\
\hline 12 & $\begin{array}{l}\text { Greenbrier View Rental Cottage on Tunis } \\
\text { Branch Road }\end{array}$ & 39.4 & 1 & 1 & 1 & 1 & 0 & 1 & 1 & 1 \\
\hline 13 & $\begin{array}{l}\text { House \#209 on Teaberry Hill Road—Front } \\
\text { yard }\end{array}$ & 39.1 & 0 & 0 & 0 & 0 & 0 & 0 & 0 & 0 \\
\hline 14 & $\begin{array}{l}\text { House \#4705 on Townsend Road-Front } \\
\text { yard }\end{array}$ & 45.8 & 0 & 0 & 0 & 0 & 1 & 0 & 0 & 0 \\
\hline 15 & $\begin{array}{l}\text { House at End of Branam Hollow } \\
\text { Road-Back yard of guest house }\end{array}$ & 38.7 & 1 & 1 & 1 & 1 & 0 & 0 & 0 & 0 \\
\hline 16 & $\begin{array}{l}\text { House \#612 on Branam Hollow } \\
\text { Road-Driveway }\end{array}$ & 35.5 & 4 & 5 & 5 & 5 & 3 & 3 & 4 & 4 \\
\hline 17 & $\begin{array}{l}\text { House \#5258 Off of Mathis Branch } \\
\text { Road-Front yard }\end{array}$ & 53.2 & 9 & 9 & 10 & 10 & 7 & 8 & 8 & 9 \\
\hline 18 & House Off of Rocky Flatts Road-Back yard & 64.0 & 5 & 5 & 6 & 6 & 5 & 5 & 6 & 6 \\
\hline 19 & House $\# 5039$ on U.S. 321-Front yard & 63.1 & -1 & 0 & 0 & 0 & 1 & 0 & 0 & -1 \\
\hline 20 & House \#4022 on U.S. $321-B a c k$ yard & 53.6 & 1 & 1 & 1 & 1 & 0 & 0 & 0 & 0 \\
\hline 21 & Young's Taxedermy Off U.S 321—Driveway & 51.8 & 0 & 0 & 0 & -1 & 1 & 0 & -1 & -1 \\
\hline
\end{tabular}




\begin{tabular}{|c|c|c|c|c|c|c|c|c|c|c|}
\hline \multirow{4}{*}{$\begin{array}{l}\text { Site } \\
\text { no. }\end{array}$} & \multirow[b]{4}{*}{ Location } & \multicolumn{9}{|c|}{ Noise level (Leq) } \\
\hline & & \multirow[b]{3}{*}{ Existing } & \multicolumn{4}{|c|}{2006} & \multicolumn{4}{|c|}{2026} \\
\hline & & & \multicolumn{2}{|c|}{ Weekday } & \multicolumn{2}{|c|}{ Weekend } & \multicolumn{2}{|c|}{ Weekday } & \multicolumn{2}{|c|}{ Weekend } \\
\hline & & & A.M. & P.M. & A.M. & P.M. & A.M. & P.M. & A.M. & P.M. \\
\hline 22 & House \#4140 on Chavis Road-Front yard & 49.3 & 0 & 0 & 0 & 0 & 0 & 0 & 0 & 0 \\
\hline 23 & Waters-Large Cemetery-Entrance & 37.6 & 0 & 0 & 0 & 0 & 0 & 0 & -1 & 0 \\
\hline 24 & House \#227 Teaberry Lane & 42.0 & 0 & 0 & 0 & 0 & 1 & 0 & 0 & 0 \\
\hline 25 & $\begin{array}{l}\text { House \#5310 on Otto Williams Road-Back } \\
\text { yard }\end{array}$ & 52.4 & 10 & 11 & 11 & 12 & 9 & 9 & 10 & 10 \\
\hline 26 & House \#327 on Costner Road-Back yard & 44.4 & 3 & 4 & 4 & 4 & 3 & 4 & 4 & 4 \\
\hline 27 & House \#430 Appletree Lane-Front yard & 40.3 & 5 & 5 & 6 & 6 & 5 & 5 & 6 & 6 \\
\hline 28 & $\begin{array}{l}\text { House on Sunshine Trail in Cobbly } \\
\text { Knob-Balcony }\end{array}$ & 41.8 & 2 & 2 & 2 & 3 & 2 & 2 & 3 & 3 \\
\hline 29 & Rental House on McKinzi Way-Front yard & 39.7 & 1 & 1 & 1 & 1 & 0 & $\mathbf{0}$ & 0 & 0 \\
\hline 30 & House on Shultz Road-Driveway & 39.6 & 1 & 1 & 1 & 1 & 0 & 0 & 0 & 0 \\
\hline 31 & Copeland Rental Cabins-Copeland Road & 47.1 & 1 & 1 & 1 & 1 & $\mathbf{0}$ & 0 & 0 & 0 \\
\hline 32 & House on The Way (last house)-Front yard & 41.1 & 0 & 0 & 0 . & 0 & 0 & 0 & 0 & 0 \\
\hline 33 & $\begin{array}{l}\text { House on The Way (second to last } \\
\text { house)-Front deck }\end{array}$ & 45.6 & 0 & 0 & -1 & 0 & 0 & 0 & 0 & 0 \\
\hline 34 & House \#429 on Appletree Lane & 50.8 & 0 & 0 & 0 & 0 & 0 & 0 & 0 & 0 \\
\hline 35 & $\begin{array}{l}\text { House at Intersection of Waters Cemetery } \\
\text { Road and Chavis Road }\end{array}$ & 49.5 & 0 & 0 & 1 & 1 & 0 & 0 & 0 & 0 \\
\hline 36 & House Off of Shultz Road & 46.9 & -1 & 0 & 0 & 0 & 1 & 0 & 0 & 0 \\
\hline 37 & $\begin{array}{l}\text { House \#916 Scenic Trail (Whistler's Watch) } \\
\text { in Cobbly Knob-Driveway }\end{array}$ & 41.3 & 1 & 1 & 1 & 1 & 0 & 0 & 0 & 0 \\
\hline 38 & $\begin{array}{l}\text { House on Sutton Place (Hidden Haven) in } \\
\text { Cobbly Knob-Driveway }\end{array}$ & 35.1 & 2 & 3 & 3 & 3 & 2 & 2 & 2 & 2 \\
\hline 39 & $\begin{array}{l}\text { Gateway Near House on Black Gum Gap } \\
\text { Road in Cobbly Knob }\end{array}$ & 54.4 & 0 & 0 & 0 & 0 & 0 & 0 & 0 & 0 \\
\hline 40 & $\begin{array}{l}\text { House (under construction) at the End of } \\
\text { Squirrel Trail in Cobbly Knob-Near } \\
\text { driveway }\end{array}$ & 36.6 & 3 & 3 & 3 & 4 & 2 & 2 & 2 & 3 \\
\hline 41 & $\begin{array}{l}\text { House (uninhabited or under construction) on } \\
\text { Rocky Flatts Road }\end{array}$ & 48.4 & 3 & 3 & 3 & 3 & 2 & 2 & 2 & 2 \\
\hline
\end{tabular}


Table L.9. Comparison of no-build alternative to interchange at U.S. 321 (both options) - 8B not opened until 8C completion

\begin{tabular}{|c|c|c|c|c|c|c|c|c|c|c|}
\hline \multirow{4}{*}{$\begin{array}{l}\text { Site } \\
\text { no. }\end{array}$} & \multirow[b]{4}{*}{ Location } & \multicolumn{9}{|c|}{ Noise level (Leq) } \\
\hline & & \multirow[b]{3}{*}{ Existing } & \multicolumn{4}{|c|}{2006} & \multicolumn{4}{|c|}{2026} \\
\hline & & & \multicolumn{2}{|c|}{ Weekday } & \multicolumn{2}{|c|}{ Weekend } & \multicolumn{2}{|c|}{ Weekday } & \multicolumn{2}{|c|}{ Weekend } \\
\hline & & & A.M. & P.M. & A.M. & P.M. & A.M. & P.M. & A.M. & P.M. \\
\hline 1 & $\begin{array}{l}\text { Foothills Pkwy Sec. } 8 A-1 \text { st Scenic } \\
\text { Overlook from U.S. } 321\end{array}$ & 47.3 & 0 & 1 & 1 & 1 & 1 & 1 & 1 & 2 \\
\hline 2 & $\begin{array}{l}\text { Foothills Pkwy Sec. } 8 \mathrm{~A}-1 \text { st Scenic } \\
\text { Overlook from I-40 }\end{array}$ & 47.3 & 0 & 1 & 1 & 1 & 1 & 1 & 1 & 2 \\
\hline 3 & $\begin{array}{l}\text { Burnett Memorial Methodist } \\
\text { Church-Pittman Center }\end{array}$ & 49.0 & 1 & 1 & 1 & 1 & 0 & 0 & 0 & 0 \\
\hline 4 & $\begin{array}{l}\text { Pittman Center Elementary } \\
\text { School-Playground }\end{array}$ & 42.6 & 1 & 1 & 1 & 1 & 0 & 0 & 0 & 0 \\
\hline 5 & Pittman Center City Hall-Parking lot & 51.3 & 0 & 0 & 1 & 0 & 1 & $\mathbf{0}$ & 0 & 1 \\
\hline 6 & $\begin{array}{l}\text { House } \# 3355 \text { between Mile Markers } 20 \text { and } \\
21 \text { on U.S. } 321 \text {-Front yard }\end{array}$ & 67.2 & -1 & -1 & 0 & 0 & 1 & 0 & -1 & -1 \\
\hline 7 & House $\# 5106$ on SR 416 -Front yard & 47.7 & 1 & 1 & 1 & 1 & 0 & 0 & 0 & 0 \\
\hline 8 & $\begin{array}{l}\text { House } \# 3010 \text { on Webb Creek Road-Front } \\
\text { yard }\end{array}$ & 40.7 & 1 & 1 & 1 & 1 & 0 & 0 & 0 & 0 \\
\hline 9 & House $\# 5005$ on SR 416 -Front yard & 62.4 & 1 & 1 & 1 & 1 & 0 & 0 & 0 & 0 \\
\hline 10 & House $\# 5215$ on SR $416-$ Front yard & 49.7 & $\mathbf{0}$ & $\mathbf{0}$ & 0 & 1 & 1 & 0 & 0 & 0 \\
\hline 11 & House \#5328 on SR 416-Front yard & 54.3 & 0 & $\mathbf{0}$ & 0 & 0 & 1 & 0 & 0 & 0 \\
\hline 12 & $\begin{array}{l}\text { Greenbrier View Rental Cottage on Tunis } \\
\text { Branch Road }\end{array}$ & 39.4 & 1 & 1 & 1 & 1 & 0 & 1 & 1 & 1 \\
\hline 13 & $\begin{array}{l}\text { House } \$ 209 \text { on Teaberry Hill Road-Front } \\
\text { yard }\end{array}$ & 39.1 & $\mathbf{0}$ & 0 & 0 & -1 & 0 & 0 & 0 & 0 \\
\hline 14 & $\begin{array}{l}\text { House } \# 4705 \text { on Townsend Road-Front } \\
\text { yard }\end{array}$ & 45.8 & -1 & 0 & 0 & 0 & 1 & 0 & 0 & 0 \\
\hline 15 & $\begin{array}{l}\text { House at End of Branam Hollow } \\
\text { Road-Back yard of guest house }\end{array}$ & 38.7 & 1 & 1 & 1 & 1 & 0 & 0 & 0 & 0 \\
\hline 16 & $\begin{array}{l}\text { House \#612 on Branam Hollow } \\
\text { Road-Driveway }\end{array}$ & 35.5 & 4 & 5 & 5 & 5 & 3 & 3 & 4 & 4 \\
\hline 17 & $\begin{array}{l}\text { House } \# 5258 \text { Off of Mathis Branch } \\
\text { Road—Front yard }\end{array}$ & 53.2 & 9 & 9 & 10 & 10 & 7 & 8 & 8 & 9 \\
\hline 18 & House Off of Rocky Flatts Road—Back yard & 64.0 & 5 & 5 & 6 & 6 & 5 & 5 & 6 & 6 \\
\hline 19 & House \#5039 on U.S. 321-Front yard & 63.1 & -1 & -1 & 0 & 0 & 1 & 0 & 0 & -1 \\
\hline 20 & House $\# 4022$ on U.S. 321 - Back yard & 53.6 & 1 & 1 & 1 & 1 & 0 & 0 & 0 & 0 \\
\hline 21 & Young's Taxedermy Off U.S 321-Driveway & 51.8 & -1 & 0 & 0 & -1 & 1 & 0 & -1 & -1 \\
\hline
\end{tabular}


Table L.9. Continued

\begin{tabular}{|c|c|c|c|c|c|c|c|c|c|c|}
\hline \multirow{4}{*}{$\begin{array}{l}\text { Site } \\
\text { no. }\end{array}$} & \multirow[b]{4}{*}{ Location } & \multicolumn{9}{|c|}{ Noise level (Leq) } \\
\hline & & \multirow[b]{3}{*}{ Existing } & \multicolumn{4}{|c|}{2006} & \multicolumn{4}{|c|}{2026} \\
\hline & & & \multicolumn{2}{|c|}{ Weekday } & \multicolumn{2}{|c|}{ Weekend } & \multicolumn{2}{|c|}{ Weekday } & \multicolumn{2}{|c|}{ Weekend } \\
\hline & & & A.M. & P.M. & A.M. & P.M. & A.M. & P.M. & A.M. & P.M. \\
\hline 22 & House $\# 4140$ on Chavis Road-Front yard & 49.3 & 0 & 0 & 0 & $\mathbf{0}$ & 0 & 0 & 0 & 0 \\
\hline 23 & Waters-Large Cemetery-Entrance & 37.6 & 0 & 0 & 0 & 0 & 0 & 0 & -1 & 0 \\
\hline 24 & House \#227 Teaberry Lane & 42.0 & 0 & -1 & 0 & 0 & 1 & 0 & 0 & 0 \\
\hline 25 & $\begin{array}{l}\text { House } \# 5310 \text { on Otto Williams Road-Back } \\
\text { yard }\end{array}$ & $52: 4$ & 10 & 11 & 11 & 12 & 9 & 9 & 10 & 10 \\
\hline 26 & House \#327 on Costner Road-Back yard & 44.4 & 3 & 4 & 4 & 4 & 3 & 4 & 4 & 4 \\
\hline 27 & House \#430 Appletree Lane-Front yard & 40.3 & 5 & 5 & 6 & 6 & 5 & 5 & 6 & 6 \\
\hline 28 & $\begin{array}{l}\text { House on Sunshine Trail in Cobbly } \\
\text { Knob-Balcony }\end{array}$ & 41.8 & 2 & 2 & 2 & 3 & 2 & 2 & 3 & 3 \\
\hline 29 & Rental House on McKinzi Way-Front yard & 39.7 & 1 & 1 & 1 & 1 & 0 & 0 & 0 & 0 \\
\hline 30 & House on Shultz Road-Driveway & 39.6 & 1 & 1 & 1 & 1 & 0 & 0 & 0 & 0 \\
\hline 31 & Copeland Rental Cabins-Copeland Road & 47.1 & 1 & 1 & 1 & 1 & 0 & 0 & 0 & 0 \\
\hline 32 & House on The Way (last house)-Front yard & 41.1 & 0 & 0 & 0 & 0 & 0 & 0 & 0 & 0 \\
\hline 33 & $\begin{array}{l}\text { House on The Way (second to last } \\
\text { house)-Front deck }\end{array}$ & 45.6 & 0 & -1 & -1 & 0 & 0 & 0 & 0 & 0 \\
\hline 34 & House \#429 on Appletree Lane & 50.8 & 0 & 0 & 0 & 0 & 0 & 0 & 0 & 0 \\
\hline 35 & $\begin{array}{l}\text { House at Intersection of Waters Cemetery } \\
\text { Road and Chavis Road }\end{array}$ & 49.5 & 0 & 0 & 0 & 1 & 0 & 0 & 0 & 0 \\
\hline 36 & House Off of Shultz Road & 46.9 & -1 & -1 & 0 & $\mathbf{0}$ & 1 & 0 & 0 & 0 \\
\hline 37 & $\begin{array}{l}\text { House \#916 Scenic Trail (Whistler's Watch) } \\
\text { in Cobbly Knob-Driveway }\end{array}$ & 41.3 & 1 & 1 & 1 & 1 & 0 & 0 & 0 & 0 \\
\hline 38 & $\begin{array}{l}\text { House on Sutton Place (Hidden Haven) in } \\
\text { Cobbly Knob-Driveway }\end{array}$ & 35.1 & 2 & 3 & 3 & 3 & 2 & 2 & 2 & 2 \\
\hline 39 & $\begin{array}{l}\text { Gateway Near House on Black Gum Gap } \\
\text { Road in Cobbly Knob }\end{array}$ & 54.4 & 0 & 0 & 0 & 0 & 0 & 0 & 0 & 0 \\
\hline 40 & $\begin{array}{l}\text { House (under construction) at the End of } \\
\text { Squirrel Trail in Cobbly Knob-Near } \\
\text { driveway }\end{array}$ & 36.6 & 3 & 3 & 3 & 4 & 2 & 2 & 2 & 3 \\
\hline 41 & $\begin{array}{l}\text { House (uninhabited or under construction) on } \\
\text { Rocky Flatts Road }\end{array}$ & 48.4 & 3 & 3 & 3 & 3 & 2 & 2 & 2 & 2 \\
\hline
\end{tabular}


Table L.10. Comparison of no-build alternative to interchange at Highway 416 (both options) $-8 \mathrm{~B}$ opened prior to $8 \mathrm{C}$ completion

\begin{tabular}{|c|c|c|c|c|c|c|c|c|c|c|}
\hline \multirow{4}{*}{$\begin{array}{l}\text { Site } \\
\text { no. }\end{array}$} & \multirow[b]{4}{*}{ Location } & \multicolumn{9}{|c|}{ Noise level (Leq) } \\
\hline & & \multirow[b]{3}{*}{ Existing } & \multicolumn{4}{|c|}{2006} & \multicolumn{4}{|c|}{2026} \\
\hline & & & \multicolumn{2}{|c|}{ Weekday } & \multicolumn{2}{|c|}{ Weekend } & \multicolumn{2}{|c|}{ Weekday } & \multicolumn{2}{|c|}{ Weekend } \\
\hline & & & A.M. & P.M. & A.M. & P.M. & A.M. & P.M. & A.M. & P.M. \\
\hline 1 & $\begin{array}{l}\text { Foothills Pkwy Sec. } 8 \text { A-1st Scenic } \\
\text { Overlook from U.S. } 321\end{array}$ & 47.3 & 0 & 1 & 1 & 1 & 1 & 1 & 1 & 2 \\
\hline 2 & $\begin{array}{l}\text { Foothills Pkwy Sec. } 8 \mathrm{~A}-1 \text { st Scenic } \\
\text { Overlook from } \mathrm{I}-40\end{array}$ & 47.3 & 0 & 1 & 1 & 1 & 1 & 1 & 1 & 2 \\
\hline 3 & $\begin{array}{l}\text { Burnett Memorial Methodist } \\
\text { Church-Pittman Center }\end{array}$ & 49.0 & 1 & 1 & 1 & 1 & 0 & 0 & 0 & 0 \\
\hline 4 & $\begin{array}{l}\text { Pittman Center Elementary } \\
\text { School-Playground }\end{array}$ & 42.6 & 1 & 1 & 1 & 1 & 0 & 0 & 0 & 0 \\
\hline 5 & Pittman Center City Hall—Parking lot & 51.3 & 2 & 1 & 2 & 1 & 2 & 1 & 2 & 2 \\
\hline 6 & $\begin{array}{l}\text { House \#3355 between Mile Markers } 20 \text { and } \\
21 \text { on U.S. } 321 \text {-Front yard }\end{array}$ & 67.2 & -1 & 0 & 0 & 0 & 1 & 0 & -1 & -1 \\
\hline 7 & House \#5106 on SR 416-Front yard & 47.7 & 1 & 1 & 1 & 1 & 0 & 0 & 0 & 0 \\
\hline 8 & $\begin{array}{l}\text { House \#3010 on Webb Creek Road-Front } \\
\text { yard }\end{array}$ & 40.7 & 1 & 1 & 1 & 1 & 0 & 0 & 0 & 0 \\
\hline 9 & House $\# 5005$ on SR 416-Front yard & 62.4 & 1 & 1 & 1 & 1 & 0 & 0 & 0 & 0 \\
\hline 10 & House $\# 5215$ on SR $416-$ Front yard & 49.7 & 3 & 2 & 2 & 3 & 4 & 2 & 3 & 3 \\
\hline 11 & House $\# 5328$ on SR $416-$ Front yard & 54.3 & 2 & 2 & 3 & 2 & 4 & 3 & 4 & 2 \\
\hline 12 & $\begin{array}{l}\text { Greenbrier View Rental Cottage on Tunis } \\
\text { Branch Road }\end{array}$ & 39.4 & 1 & 1 & 1 & 1 & 0 & 1 & 1 & 1 \\
\hline 13 & $\begin{array}{l}\text { House \#209 on Teaberry Hill Road-Front } \\
\text { yard }\end{array}$ & 39.1 & 0 & $\mathbf{0}$ & 0 & -1 & 0 & 0 & 0 & 0 \\
\hline 14 & $\begin{array}{l}\text { House } \# 4705 \text { on Townsend Road-Front } \\
\text { yard }\end{array}$ & 45.8 & 0 & 0 & 0 & $\mathbf{0}$ & 1 & $\mathbf{0}$ & 0 & 0 \\
\hline 15 & $\begin{array}{l}\text { House at End of Branam Hollow } \\
\text { Road-Back yard of guest house }\end{array}$ & 38.7 & 1 & 1 & 1 & 1 & 0 & 0 & 0 & 0 \\
\hline 16 & $\begin{array}{l}\text { House \#612 on Branam Hollow } \\
\text { Road-Driveway }\end{array}$ & 35.5 & 4 & 5 & 5 & 5 & 3 & 3 & 4 & 4 \\
\hline 17 & $\begin{array}{l}\text { House \#5258 Off of Mathis Branch } \\
\text { Road-Front yard }\end{array}$ & 53.2 & 9 & 9 & 10 & 10 & 7 & 8 & 8 & 9 \\
\hline 18 & House Off of Rocky Flatts Road—Back yard & 64.0 & 5 & 5 & 6 & 6 & 5 & 5 & 6 & 6 \\
\hline 19 & House $\# 5039$ on U.S. 321 -Front yard & 63.1 & -1 & 0 & 0 & 0 & 1 & 0 & 0 & -1 \\
\hline 20 & House \#4022 on U.S. $321-$ Back yard & 53.6 & 1 & 1 & 1 & 1 & 0 & 0 & 0 & 0 \\
\hline 21 & Young's Taxedermy Off U.S 321-Driveway & 51.8 & 0 & 0 & 0 & -1 & 1 & 0 & -1 & -1 \\
\hline
\end{tabular}




\begin{tabular}{|c|c|c|c|c|c|c|c|c|c|c|}
\hline \multirow{4}{*}{$\begin{array}{l}\text { Site } \\
\text { no. }\end{array}$} & \multirow[b]{4}{*}{ Location } & \multicolumn{9}{|c|}{ Noise level (Leq) } \\
\hline & & \multirow[b]{3}{*}{ Existing } & \multicolumn{4}{|c|}{2006} & \multicolumn{4}{|c|}{2026} \\
\hline & & & \multicolumn{2}{|c|}{ Weekday } & \multicolumn{2}{|c|}{ Weekend } & \multicolumn{2}{|c|}{ Weekday } & \multicolumn{2}{|c|}{ Weekend } \\
\hline & & & A.M. & P.M. & A.M. & P.M. & A.M. & P.M. & A.M. & P.M. \\
\hline 22 & House $\# 4140$ on Chavis Road-Front yard & 49.3 & 0 & 0 & 0 & 0 & 0 & 0 & 0 & 0 \\
\hline 23 & Waters-Large Cemetery-Entrance & 37.6 & 0 & 0 & 0 & 0 & 0 & 0 & -1 & 0 \\
\hline 24 & House \#227 Teaberry Lane & 42.0 & 0 & 0 & 0 & 0 & 1 & 0 & 0 & 0 \\
\hline 25 & $\begin{array}{l}\text { House \#5310 on Otto Williams Road-Back } \\
\text { yard }\end{array}$ & 52.4 & 10 & 11 & 11 & 12 & 9 & 9 & 10 & 10 \\
\hline 26 & House \#327 on Costner Road-Back yard & 44.4 & 3 & 4 & 4 & 4 & 3 . & 4 & 4 & 4 \\
\hline 27 & House \#430 Appletree Lane-Front yard & 40.3 & 5 & 5 & 6 & 6 & 5 & 5 & 6 & 6 \\
\hline 28 & $\begin{array}{l}\text { House on Sunshine Trail in Cobbly } \\
\text { Knob-Balcony }\end{array}$ & 41.8 & 2 & 2 & 2 & 3 & 2 & 2 & 3 & 3 \\
\hline 29 & Rental House on McKinzi Way-Front yard & 39.7 & 1 & 1 & 1 & 1 & 0 & 0 & 0 & 0 \\
\hline 30 & House on Shultz Road-Driveway & 39.6 & 1 & 1 & 1 & 1 & 0 & 0 & 0 & 0 \\
\hline 31 & Copeland Rental Cabins-Copeland Road & 47.1 & 1 & 1 & 1 & 1 & 0 & 0 & 0 & 0 \\
\hline 32 & House on The Way (last house)-Front yard & 41.1 & 0 & 0 & 0 & 0 & 0 & 0 & 0 & 0 \\
\hline 33 & $\begin{array}{l}\text { House on The Way (second to last } \\
\text { house)-Front deck }\end{array}$ & 45.6 & 0 & -1 & -1 & 0 & 1 & 0 & 0 & 0 \\
\hline 34 & House $\# 429$ on Appletree Lane & 50.8 & 0 & 0 & 0 & 0 & 0 & 0 & 0 & 0 \\
\hline 35 & $\begin{array}{l}\text { House at Intersection of Waters Cemetery } \\
\text { Road and Chavis Road }\end{array}$ & 49.5 & 0 & 0 & $\mathbf{0}$ & 1 & 0 & 0 & 0 & 0 \\
\hline 36 & House Off of Shultz Road & 46.9 & -1 & 0 & 0 & 0 & 1 & 0 & 0 & 0 \\
\hline 37 & $\begin{array}{l}\text { House \#916 Scenic Trail (Whistler's Watch) } \\
\text { in Cobbly Knob-Driveway }\end{array}$ & 41.3 & 1 & 1 & 1 & 1 & 0 & 0 & 0 & 0 \\
\hline 38 & $\begin{array}{l}\text { House on Sutton Place (Hidden Haven) in } \\
\text { Cobbly Knob-Driveway }\end{array}$ & 35.1 & 2 & 3 & 3 & 3 & 2 & 2 & 2 & 2 \\
\hline 39 & $\begin{array}{l}\text { Gateway Near House on Black Gum Gap } \\
\text { Road in Cobbly Knob }\end{array}$ & 54.4 & 0 & 0 & 0 & 0 & 0 & 0 & 0 & 0 \\
\hline 40 & $\begin{array}{l}\text { House (under construction) at the End of } \\
\text { Squirrel Trail in Cobbly Knob-Near } \\
\text { driveway }\end{array}$ & 36.6 & 3 & 3 & 3 & 4 & 2 & 2 & 2 & 3 \\
\hline 41 & $\begin{array}{l}\text { House (uninhabited or under construction) on } \\
\text { Rocky Flatts Road }\end{array}$ & 48.4 & 3 & 3 & 3 & 3 & 2 & 2 & 2 & 2 \\
\hline
\end{tabular}


Table L.11. Comparison of no-build alternative to interchange at U.S. 321 (both options)-8B opened prior to $8 \mathrm{C}$ completion

\begin{tabular}{|c|c|c|c|c|c|c|c|c|c|c|}
\hline \multirow{4}{*}{$\begin{array}{l}\text { Site } \\
\text { no. }\end{array}$} & \multirow[b]{4}{*}{ Location } & \multicolumn{9}{|c|}{ Noise level (Leq) } \\
\hline & & \multirow[b]{3}{*}{ Existing } & \multicolumn{4}{|c|}{2006} & \multicolumn{4}{|c|}{2026} \\
\hline & & & \multicolumn{2}{|c|}{ Weekday } & \multicolumn{2}{|c|}{ Weekend } & \multicolumn{2}{|c|}{ Weekday } & \multicolumn{2}{|c|}{ Weekend } \\
\hline & & & A.M. & P.M. & A.M. & P.M. & A.M. & P.M. & A.M. & P.M. \\
\hline 1 & $\begin{array}{l}\text { Foothills Pkwy Sec. } 8 \mathrm{~A}-1 \text { st Scenic } \\
\text { Overlook from U.S. } 321\end{array}$ & 47.3 & 0 & 1 & 1 & 1 & 1 & 1 & 1 & 2 \\
\hline 2 & $\begin{array}{l}\text { Foothills Pkwy Sec. 8A-1st Scenic } \\
\text { Overlook from } 1-40\end{array}$ & 47.3 & 0 & 1 & 1 & 1 & 1 & 1 & 1 & 2 \\
\hline 3 & $\begin{array}{l}\text { Burnett Memorial Methodist } \\
\text { Church-Pittman Center }\end{array}$ & 49.0 & 1 & 1 & 1 & 1 & 0 & 0 & 0 & 0 \\
\hline 4 & $\begin{array}{l}\text { Pittman Center Elementary } \\
\text { School-Playground }\end{array}$ & 42.6 & 1 & 1 & 1 & 1 & 0 & 0 & 0 & 0 \\
\hline 5 & Pittman Center City Hall-Parking lot & 51.3 & 0 & 0 & 1 & 0 & 1 & 0 & 0 & 1 \\
\hline 6 & $\begin{array}{l}\text { House \#3355 between Mile Markers } 20 \text { and } \\
21 \text { on U.S. } 321 \text {-Front yard }\end{array}$ & 67.2 & 0 & 0 & 0 & 0 & 1 & 0 & -1 & 0 \\
\hline 7 & House \#5106 on SR 416-Front yard & 47.7 & 1 & 1 & 1 & 1 & 0 & 0 & 0 & 0 \\
\hline 8 & $\begin{array}{l}\text { House \#3010 on Webb Creek Road-Front } \\
\text { yard }\end{array}$ & 40.7 & 1 & 1 & 1 & 1 & 0 & 0 & 0 & 0 \\
\hline 9 & House $\# 5005$ on SR 416 -Front yard & 62.4 & 1 & 1 & 1 & 1 & 0 & 0 & 0 & 0 \\
\hline 10 & House $\# 5215$ on SR 416 -Front yard & 49.7 & 0 & 0 & 0 & 0 & 1 & 0 & 0 & 0 \\
\hline 11 & House $\# 5328$ on SR $416-$ Front yard & 54.3 & 0 & 0 & 0 & 0 & 1 & 0 & 0 & 0 \\
\hline 12 & $\begin{array}{l}\text { Greenbrier View Rental Cottage on Tunis } \\
\text { Branch Road }\end{array}$ & 39.4 & 1 & 1 & 1 & 1 & 0 & 1 & 1 & 1 \\
\hline 13 & $\begin{array}{l}\text { House \#209 on Teaberry Hill Road-Front } \\
\text { yard }\end{array}$ & 39.1 & 0 & 0 & 1 & 0 & 0 & 0 & 1 & 1 \\
\hline 14 & $\begin{array}{l}\text { House \#4705 on Townsend Road-Front } \\
\text { yard }\end{array}$ & 45.8 & 0 & 0 & 0 & 0 & 1 & 0 & 1 & 1 \\
\hline 15 & $\begin{array}{l}\text { House at End of Branam Hollow } \\
\text { Road-Back yard of guest house }\end{array}$ & 38.7 & 1 & 1 & 1 & 1 & 0 & 0 & 0 & 0 \\
\hline 16 & $\begin{array}{l}\text { House \#612 on Branam Hollow } \\
\text { Road-Driveway }\end{array}$ & 35.5 & 4 & 5 & 5 & 5 & 3 & 3 & 4 & 4 \\
\hline 17 & $\begin{array}{l}\text { House \#5258 Off of Mathis Branch } \\
\text { Road-Front yard }\end{array}$ & 53.2 & 9 & 9 & 10 & 10 & 7 & 8 & 8 & 9 \\
\hline 18 & House Off of Rocky Flatts Road-Back yard & 64.0 & 5 & 5 & 6 & 6 & 5 & 5 & 6 & 6 \\
\hline 19 & House $\# 5039$ on U.S. 321 -Front yard & 63.1 & 0 & 0 & 0 & 0 & 1 & 0 & 0 & 0 \\
\hline 20 & House $\# 4022$ on U.S. $321-$ Back yard & 53.6 & 1 & 1 & 1 & 1 & 0 & 0 & 0 & 0 \\
\hline 21 & Young's Taxedermy Off U.S 321-Driveway & 51.8 & 0 & 0 & $\mathbf{0}$ & -1 & 1 & 0 & 0 & 0 \\
\hline
\end{tabular}




\begin{tabular}{|c|c|c|c|c|c|c|c|c|c|c|}
\hline \multirow{4}{*}{$\begin{array}{l}\text { Site } \\
\text { no. }\end{array}$} & \multirow[b]{4}{*}{ Location } & \multicolumn{9}{|c|}{ Noise level (Leq) } \\
\hline & & \multirow[b]{3}{*}{ Existing } & \multicolumn{4}{|c|}{2006} & \multicolumn{4}{|c|}{2026} \\
\hline & & & \multicolumn{2}{|c|}{ Weekday } & \multicolumn{2}{|c|}{ Weekend } & \multicolumn{2}{|c|}{ Weekday } & \multicolumn{2}{|c|}{ Weekend } \\
\hline & & & A.M. & P.M. & A.M. & P.M. & A.M. & P.M. & A.M. & P.M. \\
\hline 22 & House $\# 4140$ on Chavis Road-Front yard & 49.3 & $\mathbf{0}$ & 0 & $\mathbf{0}$ & 0 & 0 & 0 & 0 & 0 \\
\hline 23 & Waters-Large Cemetery-Entrance & 37.6 & 0 & 0 & 0 & 0 & 0 & 0 & -1 & 0 \\
\hline 24 & House \#227 Teaberry Lane & 42.0 & 1 & 0 & 0 & 0 & 1 & 0 & $\mathbf{0}$ & 0 \\
\hline 25 & $\begin{array}{l}\text { House \#5310 on Otto Williams Road-Back } \\
\text { yard }\end{array}$ & 52.4 & 10 & 11 & 11 & 12 & 9 & 9 & 10 & 10 \\
\hline 26 & House $\# 327$ on Costner Road-Back yard & 44.4 & 3 & 4 & 4 & 4 & 3 & 4 & 4 & 4 \\
\hline 27 & House \#430 Appletree Lane-Front yard & 40.3 & 5 & 5 & 6 & 6 & 5 & 5 & 6 & 6 \\
\hline 28 & $\begin{array}{l}\text { House on Sunshine Trail in Cobbly } \\
\text { Knob-Balcony }\end{array}$ & 41.8 & 2 & 2 & 2 & 3 & 2 & 2 & 3 & 3 \\
\hline 29 & Rental House on McKinzi Way-Front yard & 39.7 & 1 & 1 & 1 & 1 & 0 & 0 & 0 & 0 \\
\hline 30 & House on Shultz Road-Driveway & 39.6 & 1 & 1 & 1 & 1 & 0 & 0 & 0 & 0 \\
\hline 31 & Copeland Rental Cabins-Copeland Road & 47.1 & 1 & 1 & 1 & 1 & 0 & 0 & 0 & 0 \\
\hline 32 & House on The Way (last house)-Front yard & 41.1 & 0 & 0 & 0 & 0 & 0 & 0 & 0 & 0 \\
\hline 33 & $\begin{array}{l}\text { House on The Way (second to last } \\
\text { house)-Front deck }\end{array}$ & 45.6 & 1 & 0 & 0 & 0 & 1 & 0 & 0 & 0 \\
\hline 34 & House \#429 on Appletree Lane & 50.8 & 0 & 0 & 0 & 0 & 0 & 0 & 0 & 0 \\
\hline 35 & $\begin{array}{l}\text { House at Intersection of Waters Cemetery } \\
\text { Road and Chavis Road }\end{array}$ & 49.5 & 0 & 0 & $\mathbf{0}$ & 1 & 0 & 0 & 0 & 0 \\
\hline 36 & House Off of Shultz Road & 46.9 & 0 & 0 & 0 & 0 & 1 & 0 & 0 & 1 \\
\hline 37 & $\begin{array}{l}\text { House \#916 Scenic Trail (Whistler's Watch) } \\
\text { in Cobbly Knob-Driveway }\end{array}$ & 41.3 & 1 & 1 & 1 & 1 & 0 & 0 & 0 & 0 \\
\hline 38 & $\begin{array}{l}\text { House on Sutton Place (Hidden Haven) in } \\
\text { Cobbly Knob-Driveway }\end{array}$ & 35.1 & 2 & 3 & 3 & 3 & 2 & 2 & 2 & 2 \\
\hline 39 & $\begin{array}{l}\text { Gateway Near House on Black Gum Gap } \\
\text { Road in Cobbly Knob }\end{array}$ & 54.4 & 0 & 0 & $\mathbf{0}$ & 0 & 0 & 0 & 0 & 0 \\
\hline 40 & $\begin{array}{l}\text { House (under construction) at the End of } \\
\text { Squirrel Trail in Cobbly Knob-Near } \\
\text { driveway }\end{array}$ & 36.6 & 3 & 3 & 3 & 4 & 2 & 2 & 2 & 3 \\
\hline 41 & $\begin{array}{l}\text { House (uninhabited or under construction) on } \\
\text { Rocky Flatts Road }\end{array}$ & 48.4 & 3 & 3 & 3 & 3 & 2 & 2 & 2 & 2 \\
\hline
\end{tabular}




\title{
Appendix M
}

\section{AESTHETIC RESOURCES}

\author{
Carl Petrich \\ Oak Ridge National Laboratory \\ Oak Ridge, Tennessee
}

August 1995

Part 1: Aesthetic Resource Methods . . . . . . . . . . . . . . . . . . . . M-3

Jack Ranney

Joint Institute for Energy and Environment

Knoxville, Tennessee

April 1997

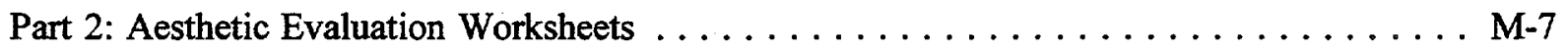




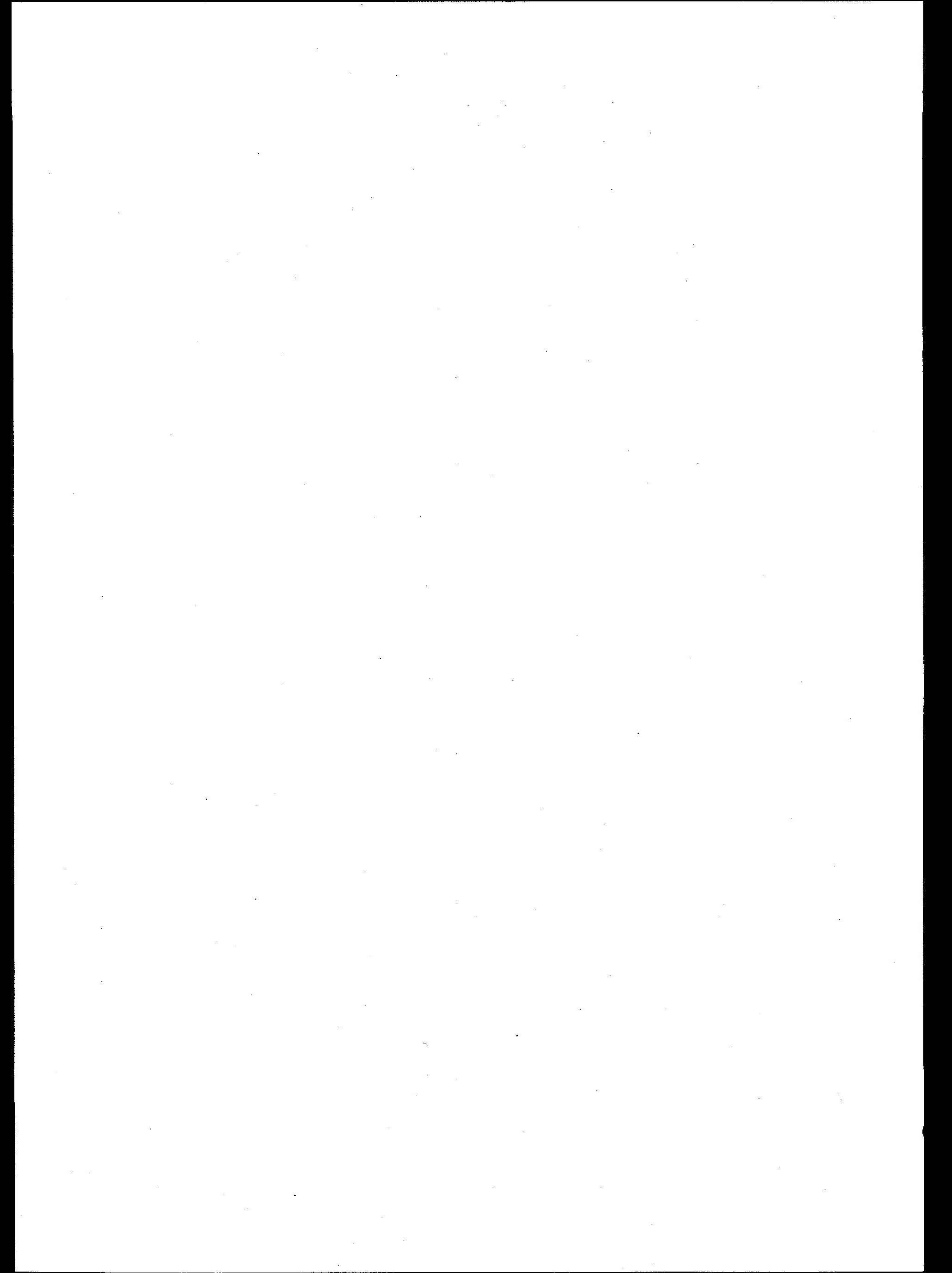




\title{
PART 1
}

With shrinking maintenance funds available to construct overlooks and keep scenic vistas open, it is paramount to select for establishment and long-term maintenance only those vistas with the highest potential for pleasing the parkway traveler. While there are no specific studies of viewer preferences along the existing sections of the Foothills Parkway, there are recent studies for the Blue Ridge Parkway of visitor preferences and maintenance regimes to support preferred vistas (Noe and Hammitt 1988).

Hammitt (1988) determined that the scenic vistas travelers on the Blue Ridge Parkway most prefer are those with water (ponds, fast-moving streams, rivers, waterfalls) in the landscape. Motorists least preferred vistas in which the foreground and midground woody vegetation had grown to partially block the views. The importance of identifying and selecting viewing points where vegetation can and will be maintained effectively and inexpensively is clear.

Based on the southern section of the Blue Ridge Parkway (i.e., those areas most nearly like Section $8 \mathrm{~B}$ of the Foothills Parkway), Hammitt found that, other than water scenes, those vistas offering open views of a series of receding mountain ridges, usually three or more in depth, were most preferred. These typically include mountain peaks and valleys that suggest smaller, dissected landscape units that are inviting to the curious viewer. Vistas of this type foster what is known as involvement. An involving scene draws the observer into it to find out more than what is only partially revealed from the observer's vantage point (Herzog 1984).

The next most preferred vista type was characterized by mountain valleys containing farms or rural community development. The third-favored scenes were of one or two background mountain ridges in which the vistas are partially blocked by foreground trees and shrubs. Hammitt interpreted the findings as viewers not minding some vegetation in the foreground as long as the farground view of the mountains is not blocked. Preference declines as foreground foliage increases. The least preferred landscape type was of scenes with only one mountain ridge that occupied three-fourths of the mid-farground view. Hammitt interpreted these scenes to offer the viewer little opportunity for further visual involvement.

Looking at more scenic preferences from travelers along the entire stretch of the Blue Ridge Parkway, Hammitt produced a Vista Preference Typology for the parkway. By proxy and the lack of other sources of information, this typology can guide evaluation of the scenic potential along Section $8 \mathrm{~B}$ of the Foothills Parkway. Hammitt's data suggest that parkway visitor's preferences will fall along a continuum as follows:

\author{
Most Preferred \\ rapidly moving water \\ stationary water \\ mountains with several ridges \\ pastoral development \\ mountains with one ridge \\ unmaintained vegetation
}

Least Preferred

In a later study, Hammitt and Patterson (1990) delimited nine major classifications of scenic landscapes viewable from overlooks along the Blue Ridge Parkway. These vary little from the 
typology elicited in the 1988 work, substantiating the earlier findings. Pastoral development is more accurately classified as scenes characterized by "broad, rolling plateau valleys; a mosaic of pastures, farms, and hardwood forests." Less desired were scenes characterized by grassy fields and open valleys.

Hammitt also found that opening vistas where vegetation blocks more than half of the view can greatly enhance the visual preference for such vistas. Viewers appear to tolerate vegetation blocking a small portion of vistas, and may even prefer a small amount of low, foreground vegetation. A certain amount of such growth might provide vista framing, flowering shrubs, and bird and small mammal habitat. A mixture of enclosed and open vistas might provide landscape diversity, also a desired management objective. Whether foreground vegetation should be removed depends on the whole set of scenic vistas provided tourists in a given region, with "region" being based, at least in part, on visitation patterns (Wellman et al. 1988).

Those who grew up (until age 16) in rural areas preferred the open vistas more than those who grew up in cities (Hampe 1988). In another study of the Blue Ridge Parkway, Noe (1988) found that most tourists believed that they needed to pull off, stop, and leave their vehicles to have an adequate sightseeing experience. For them, taking photographs was a dominant experience.

Tourists also enjoy the full range of scenery from natural cliffs, peaks, and streams to the more man-influenced rural scenes such as farms and fields.

Wellman et al. (1988) concluded that perceived visual impacts to a landscape do not necessarily increase or decrease linearly with actual changes in the physical environment. They believe that scenic beauty acts on the margin; that is, a small amount of impact can be perceived as significantly deleterious, after which additional damage seems to create little further negative perceptions. From the positive perspective, a little vegetation management on selected vistas might substantially enhance their perceived scenic beauty, while extensive and expensive work on heavily overgrown sites may effect little improvement in visitor appreciation.

All these factors are critical in identifying, at the front end, those sites most appropriate for supporting scenic overlooks or otherwise managed viewing areas. Open vistas should be kept as central and integral experiences of the parkway visitor. Historically, parkways have been designed to facilitate "making pictures as you drive" (Forgery 1989). The average parkway users will receive most of their aesthetic experiences at scenic vistas. About a third of the visiting public heavily uses pull-offs and overlooks. A strategic concentration of resources on these pull-offs would have the maximum effect for an investment of resources.

Evaluation of Section 8B's aesthetic resources needs to be considered in the context of the potential of the larger parkway experience. While only portions of the parkway have been built, and not all portions have yet been evaluated, there are elements of the likely contiguous experience that can inform the analysis of Section $8 \mathrm{~B}$. The quality of individual views from Section $8 \mathrm{~B}$ needs to be assessed in terms of the relative frequency and quality among all views available or potentially available along the parkway. Where a given view falls in the sequence of all views experienced depends on driving direction and is vitally important in assessing its quality. Certain assumptions have to be made about how unbuilt portions of the parkway will likely be designed and about how much of the parkway most drivers are likely to drive at one time.

Aesthetic enjoyment of driving the parkway will depend on visual inputs that lead to a satisfying sense of orientation and variety. The well-designed parkway will signal the traveler with simple, understandable, and anticipated low-order systems of visual stimuli that lead to increasing levels of 
orientation. The traveler should easily grasp what the environment observed is about, something of the landscape's evolution, where he or she is in that environment, and progress made along the route. The critical element in this grammar is that, beyond a certain range, the messages can be so often repeated that they become banal, visual clichés. "The optimal combination of visual messages must include a share of redundant (repetitive, anticipated) messages, to provide orientation, and a share of highly informative (new) messages, to provide variety" (Lozano 1988). Lozano describes this ideal combination of information in a variety of ways: "redundancy and new information, low order and high order, immediate understanding and incomplete understanding, orientation and variety." "The parkway must allow the natural environment to reveal its most critical, new information in a considered sequence relative to the other information it communicates. High-quality views build on each other and are complemented and reinforced by appropriate sequences and frequencies of the other, lower-order visual experiences offered.

In the segment descriptions in Sect. 3.8.4, for example, the views from Webb Mountain certainly are the likely highlight of the Section $8 \mathrm{~B}$ aesthetic experience and, possibly of the entire parkway. At Webb Mountain, the parkway is at one of its highest elevations, overlooking one of its deepest valleys, and is at its nearest proximity to the spine of the Great Smoky Mountains. The views selected and designed that lead up to it from either direction will determine, in part, the quality of travelers' experiences. From Section 8A, only two views of the GSMNP are remotely comparable, and those are from a much farther distance: more panoramic but less dramatic. Section $8 \mathrm{C}$ has not been surveyed for this analysis. Sections $8 \mathrm{~A}$ and $8 \mathrm{D}$ (and much of $8 \mathrm{~B}$ ) offer substantial travel through oak-pine forest, with primarily near, foreground views of the adjacent forest. Only in the area of Flat Branch in 8D does the ROW reach the crest of Cove Mountain and offer views toward Mount LeConte and the GSMNP (National Park Service 1995). Thus, within the context of travel from Wear Valley to I-40 in the north and east, the importance of Webb Mountain becomes clearer than when considered only from the $8 \mathrm{~B}$ assessment.

The designer needs to consider whether proximate, ancillary views reveal too much of what is likely to be seen so that the Webb Mountain views are somewhat anticlimactic. Have secondary views provided sufficient glimpses of the grandeur to come in a way that teases the observer? Has there been tension created by a competing view revealed earlier? Surely, the view from Webb Mountain captures the expectations of what one unfamiliar with the Great Smoky Mountains might expect based on the region's popularized image. Because of this heightened anticipation, the importance of its presentation (identification, design, sequencing, development, maintenance) is correspondingly heightened.

Because travelers (especially drivers) are free from having to pay attention to the normal load of decisionmaking while on a parkway, and because of the slow speeds and low traffic volumes on a parkway, it is the designer's role to create an adequate range of visual complexity to sustain travelers' interest. As with any artistic creation, the designer seeks to provide a clear organization of sequences and a meaningful visual composition (Hornbeck and Okerlund 1973): "Sequential experience is the phrasing and composition of what the driver sees." To avoid monotony and to

\footnotetext{
'Mathematician Alfred North Whitehead laid out these ideas most powerfully in his Principles of Natural Knowledge (1925, New York, Cambridge University Press): “A rhythm involves a pattern, and to that extent is always self-identical. But no thythm can be a mere pattern; for the rhythmic quality depends equally upon the differences involved in each exhibition of the pattern. The essence of thythm is the fusion of sameness and novelty, so that the whole never loses the essential unity of the pattern, while the parts exhibit the contrast arising from the novelty of their detail. A mere recurrence kills rhythm as surely as does a mere confusion of detail."
} 
provide interest, contrast, and continuity for the traveler's experience, the designer seeks to structure a meaningful, continuous sequence. 
PART 2 


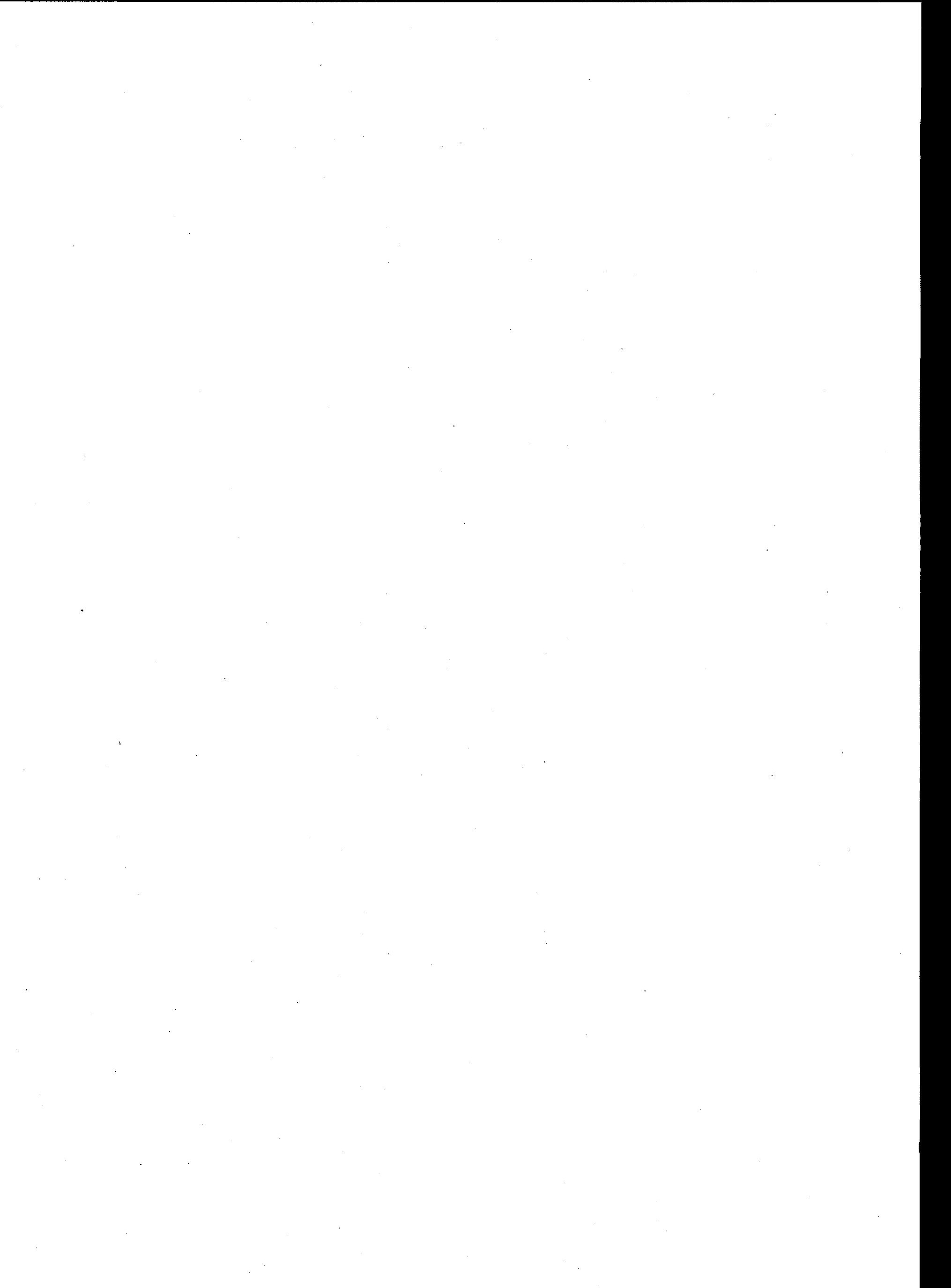




\section{Aesthetic Experience Worksheet Foothills Parkway Extension 8-B *** Views From the Parkway Extension *** \\ View 'A', Little Pigeon River, 1-400 to 1-680}

\begin{tabular}{|c|}
\hline Presentation of View (pick one) \\
Best - View along outside of moderate road curve with long \\
line of sight on road; view for $6+$ seconds at speed limit \\
Better - View along outside of moderate/sharp road curve with \\
moderate line of sight on road, view $4-6$ seconds at \\
speed limit \\
Good - View along straight roadside with long to moderate tine \\
of sight on road; view $3-4$ seconds at speed limit \\
Fair - Along tight curves and short lines of sight on road; \\
view less than 3 seconds at speed limit
\end{tabular}

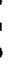

\begin{tabular}{|l|}
\hline Special Experience Opportunity (pick one) \\
I. Fall color; spring blooms; water sounds; cool in summer; \\
very special lighting effects \\
II.* Morning or ovening back/side lighting; mists; winter \\
ice/frosts; deep woods; cool wet smells \\
III. Wildlife viewing (birds, deer, etc.); special geology; cool \\
woods smell; big trees; unusual vegetation; noticeable \\
ecological processes \\
IV. Mostly normal mountain vegetation; little unusual lighting \\
effects or sensory experiences expected \\
V. Blinding aun; heavy fog; bad smells; bad traffic situation; \\
noisy; strong winds; dead and dying vegetation; hot in \\
summer; trash in view
\end{tabular}

Choices are marked with an asterisk.

\begin{tabular}{|c|c|}
\hline \multicolumn{2}{|c|}{ Quality of View (pick one) } \\
\hline \multicolumn{2}{|c|}{$\begin{array}{c}\text { Very Best - Water scenes with long views of series of } \\
\text { receding mountain ridges and valleys }\end{array}$} \\
\hline Best & $\begin{array}{l}\text { - Long viows of receding mountain ridges or } \\
\text { water scenes but not both; close views of } \\
\text { wooded mountain streams }\end{array}$ \\
\hline Better* & $\begin{array}{l}\text { - Long but partially blocked views of mountain } \\
\text { ridges or water scenes; more midground ridges } \\
\text { than long view }\end{array}$ \\
\hline & $\begin{array}{l}\text { - Midground views of opposing ridges; rustic } \\
\text { valloys, littlo to no long views }\end{array}$ \\
\hline Fair & $\begin{array}{l}\text { - Midground to foreground views of opposing } \\
\text { ridges, close viows of roads, traffic; } \\
\text { development may be present }\end{array}$ \\
\hline
\end{tabular}

$\checkmark$
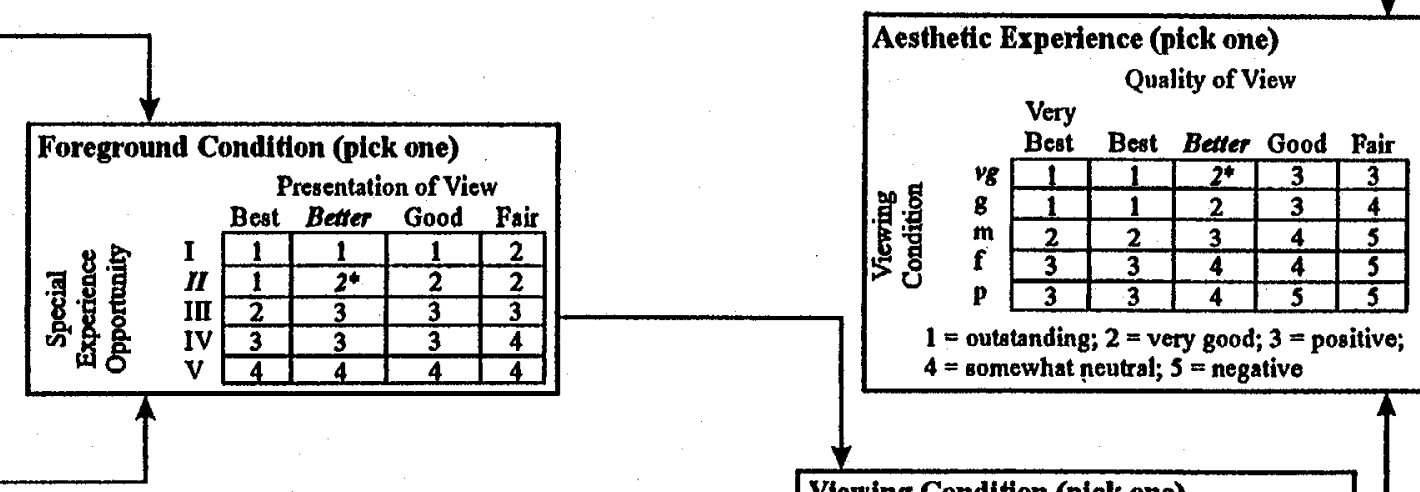

$1=$ outstanding; 2 = very good; 3 = positive; $4=$ somewhat neutral; $5=$ negative

Opportunity of View (pick one)

$A$.* Horizontal view angle $>180^{\circ}$; vertical view angle $>20^{\circ}$; pull over present

B. Horizontal view angle $90-180^{\circ}$; vertical view angle $15-20^{\circ}$; pull over present

C. Horizontal view angle $45-90^{\circ}$; vertical angle $10-15^{\circ}$; pull over available; larger viowing angles with no pull over available

D. Horizontal view angle $30-45^{\circ}$; no pull over available

B. Horizontal view angle $<30^{\circ}$; no pull over

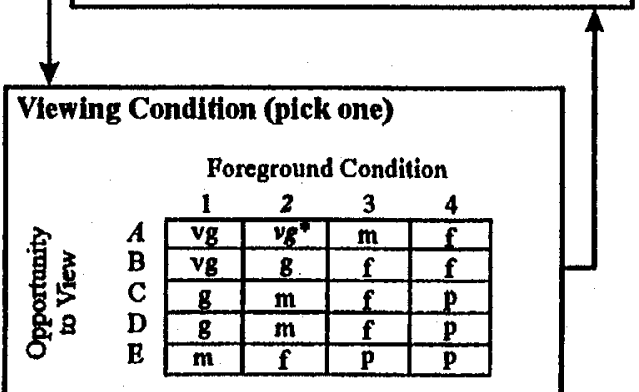

$v g=$ very good; $g=$ good; $m=$ moderate; $p=$ poor 


\section{Aesthetic Experience Worksheet Foothills Parkway Extension 8-B *** Views From the Parkway Extension ***}

\section{View 'B', Tunis Branch, 2-170}

Best - View along outside of moderate road curve with long line of sight on road; view for $6+$ seconds at speed limit Better - View along outside of moderate/sharp road curve with moderate line of sight on road, view 4-6 seconds at speed limit

Good* - Viow along straight roadside with long to moderate lino of sight on road; view 3-4 seconds at speed limit Fair - Along tight curves and short lines of sight on road, view less than 3 seconds at speed limit

Special Experience Opportunity (pick one)

Fall color, spring blooms; water sounds; cool in summer very special lighting effect

II. Morning or evening back/side lighting; mists; winter ice/frosts; deep woods; cool wet smells

III.* Wildlife viewing (birds, deer, etc.); apecial geology; cool woods smell; big trees; unusual vegetation; noticeable ecological processes

IV. Mostly normal mountain vegetation; little unusual lighting effects or sensory experiences expected

V Blinding sun; heavy fog; bad smells; bad traffic situation; noigy; strong winds; dead and dying vegetation; hot in summer; trash in view

Choices are marked with an asterisk.

\begin{tabular}{|c|c|}
\hline Qualit & of View (pick one) \\
\hline Very Be & $\begin{array}{l}\text { - Water scenes with long viows of series of } \\
\text { receding mountain ridges and valleys }\end{array}$ \\
\hline & $\begin{array}{l}\text { - Long views of receding mountain ridges or } \\
\text { water scenes but not both; close viows of } \\
\text { wooded mountain stroams }\end{array}$ \\
\hline Better & $\begin{array}{l}\text { - Long but partially blocked views of mountain } \\
\text { ridges or water scenes; more midground ridges } \\
\text { than long view }\end{array}$ \\
\hline & $\begin{array}{l}\text { - Midground viows of opposing ridges; rustic } \\
\text { valloys, little to no long views }\end{array}$ \\
\hline & $\begin{array}{l}\text { - Midground to foreground views of opposing } \\
\text { ridges, close views of roads, traffic; } \\
\text { development may be present }\end{array}$ \\
\hline
\end{tabular}

$\downarrow$

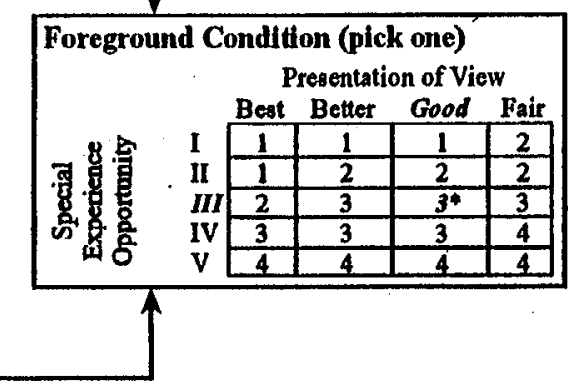

Acsthetic Experience (pick one)
Quality of Viow

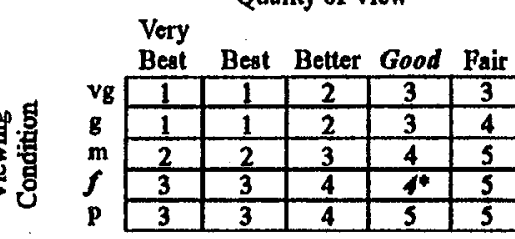

1 = outstanding; 2 = very good; 3 = positive;

Opportunity of Vlew (pick one)

A. Horizontal view angle $>180^{\circ}$; vertical view angle $>20^{\circ}$; pull ver present

B. Horizontal view angle $90-180^{\circ}$; vertical view angle $15-20^{\circ}$; pull over present

C.* Horizontal view anglo $45-90^{\circ}$; vertical anglo $10-15^{\circ}$; pull over available; larger viewing angles with no pull over available

D. Horizontal view angle $30-45^{\circ}$; no pull over available

B. Horizontal view angle $<30^{\circ}$; no pull over
$4=$ somewhat neutral; $5=$ negative

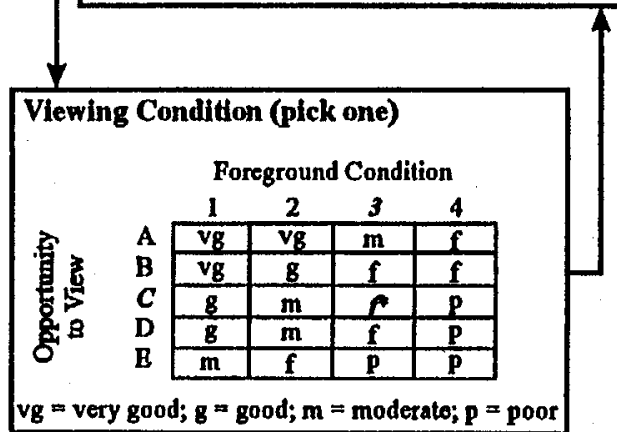

$v g=$ very good; $g=$ good; $m=$ moderate; $p=$ poor 


\section{Aesthetic Experience Worksheet Foothills Parkway Extension 8-B **** Views From the Parkway Extension ***}

\section{View '1', Webb Creek Valley, 2-380 to 2-970}

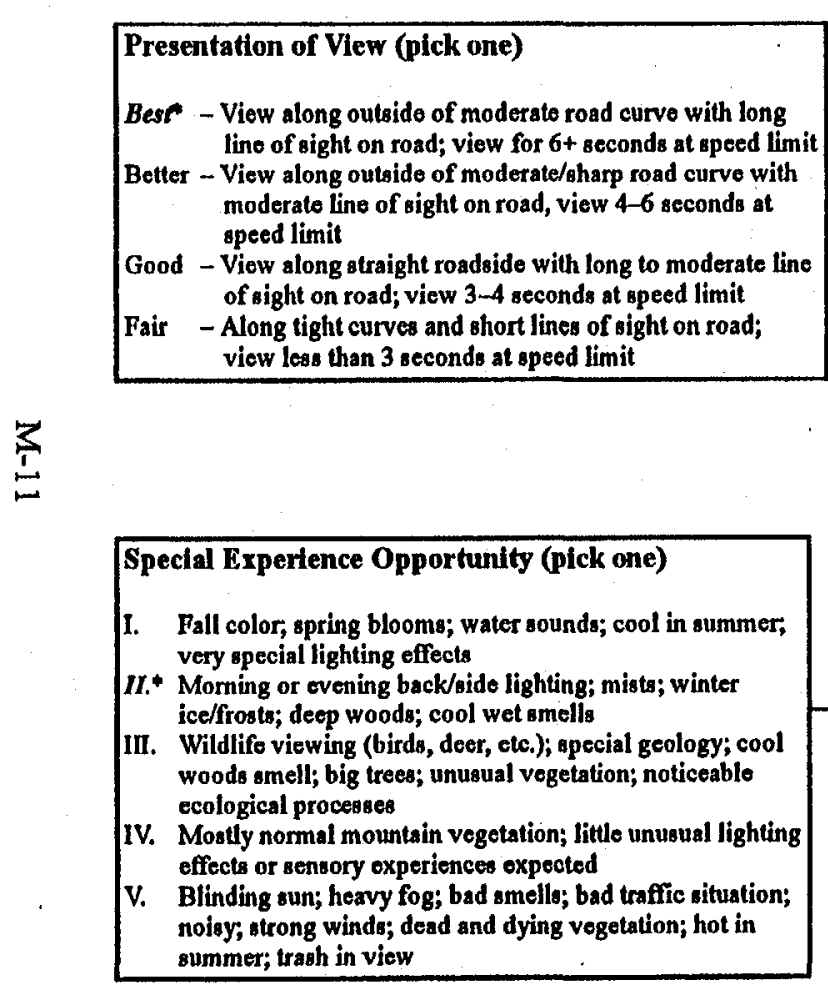

Choices are marked with an asterisk.

$\checkmark$
pull over present availablo

\begin{tabular}{|c|c|}
\hline \multicolumn{2}{|c|}{ Quality of View (pick one) } \\
\hline \multicolumn{2}{|c|}{$\begin{array}{l}\text { Very Best - Water scenes with long views of series of } \\
\text { receding mountain ridges and valleys }\end{array}$} \\
\hline & $\begin{array}{l}\text { - Long viows of receding mountain ridges or } \\
\text { water scenes but not both; close views of } \\
\text { wooded mountain streams }\end{array}$ \\
\hline Better" & $\begin{array}{l}\text { - Long but partially blocked viows of mountain } \\
\text { ridges or water scenes; more midground ridges } \\
\text { than long view }\end{array}$ \\
\hline & $\begin{array}{l}\text { - Midground views of opposing ridges; rustic } \\
\text { valleys; little to no long views }\end{array}$ \\
\hline Fair & $\begin{array}{l}\text { - Midground to foreground views of opposing } \\
\text { ridges, close viows of roads, traffic; } \\
\text { development may be prosent }\end{array}$ \\
\hline
\end{tabular}
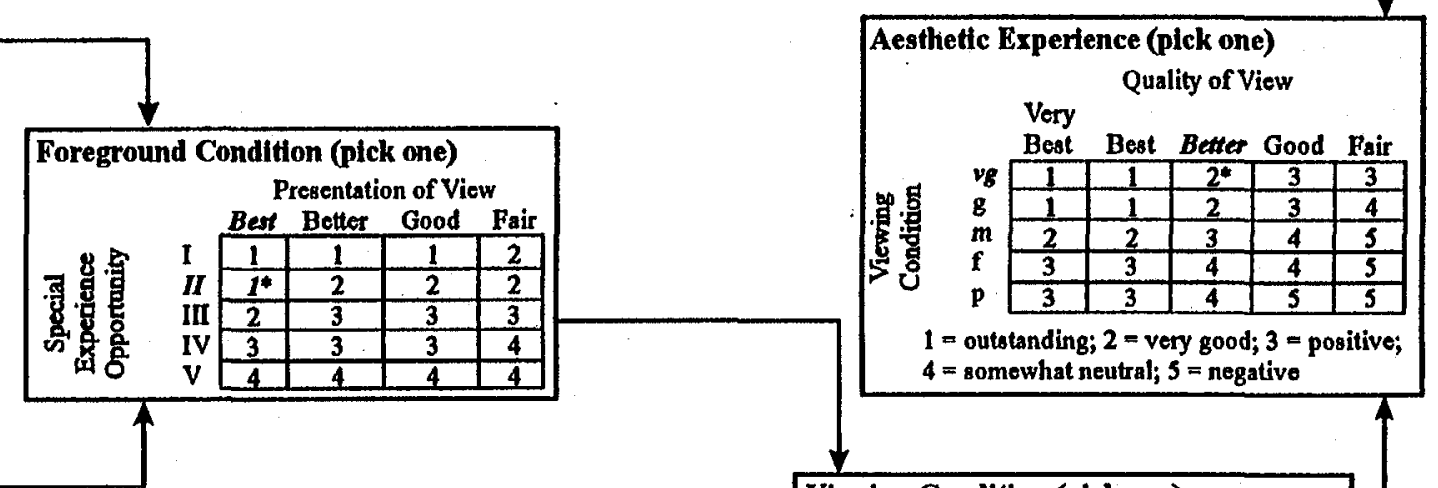

Opportunity of Vlew (pick one)

A. Horizontal view angle $>180^{\circ}$; vertical view angle $>20^{\circ}$; pull

B. Horizontal view anglo $90-180^{\circ}$; vertical view angle 15-20

C. Horizontal view anglo $45-90^{\circ}$; vertical anglo $10-15^{\circ}$; pull over available; larger viewing angles with no pull over

D. Horizontal view angle $30-43^{\circ}$; no pull over available

B. Horizontal view anglo $<30^{\circ}$; no pull over 


\section{Aesthetic Experience Worksheet}

Foothills Parkway Extension 8-B *** Views From the Parkway Extension ***

\section{View 'D', Soak Ash Creek, 3-320 to 3-550 (Serial View)}

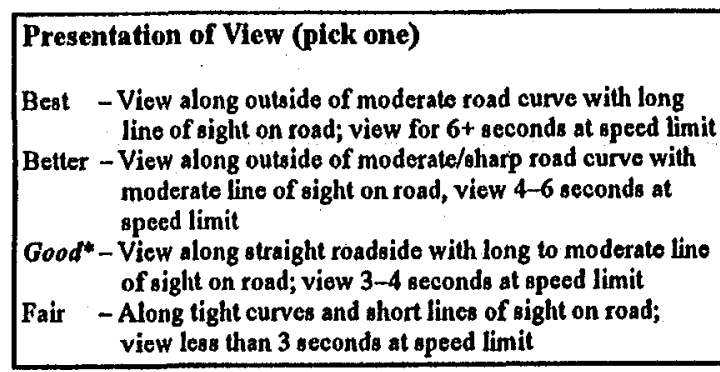

Special Experience Opportunity (pick one)

Fall color, spring blooms; water sounds; cool in summer, very special lighting effects

Morning or evening back/side lighting; mistw; winter ice/frosts; deep woods; cool wet smells

III. Wildlife viewing (birds, deer, etc.); special geology; cool woods smell; big trees; unusual vegetation; noticeablo ecological processe

IV.* Mootly normal mountsin vegetation; title unusual lighting effects or sensory experiences expected

V. Blinding sun; heavy fog; bad emells; bad traffic situation; noiay; strong winds; dead and dying vegetation; hot in summer; trash in view

Choices are marked with an asterisk.

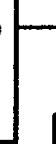

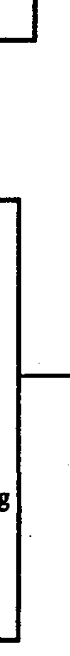
over present pull over present available

\begin{tabular}{|c|c|}
\hline Quallt & of View (pick one) \\
\hline Very $B$ & $\begin{array}{l}\text { - Water scenes with long viows of series of } \\
\text { receding mountain ridges and valloys }\end{array}$ \\
\hline Best & $\begin{array}{l}\text { - Long viows of receding mountain ridges or } \\
\text { water scenes but not both; close viows of } \\
\text { wooded mountain streams }\end{array}$ \\
\hline Better & $\begin{array}{l}\text { - Long but partially blocked views of mountain } \\
\text { ridges or water scenes; more midground ridges } \\
\text { than long view }\end{array}$ \\
\hline & $\begin{array}{l}\text { - Midground views of opposing ridges; rustio } \\
\text { valleys, littlo to no long views }\end{array}$ \\
\hline Fair & $\begin{array}{l}\text { - Midground to foreground views of opposing } \\
\text { ridges, close views of roads, traffie; } \\
\text { development may be present }\end{array}$ \\
\hline
\end{tabular}

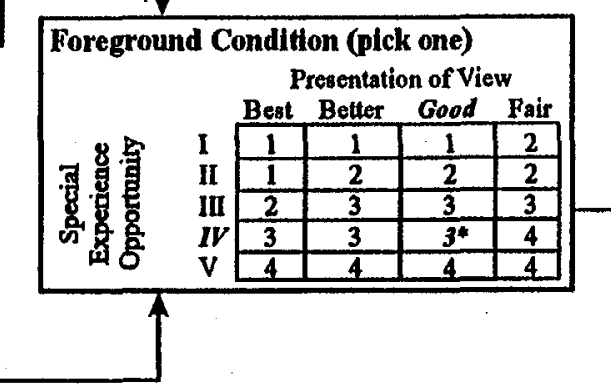

\section{Aesthetic Experience (pick one) \\ Quality of View}

Very

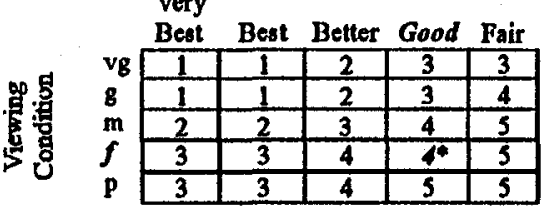

$1=$ outstanding; 2 = very good; 3 = positive; $4=$ somewhat neutral; $5=$ negative

Opportunity of Vlew (pick one)

A. Horizontal view angle $>180^{\circ}$; vertical view angle $>20^{\circ}$; pull

. Horizontal view angle $90-180^{\circ}$; vertical view anglo $15-20^{\circ}$;

C.* Horizontal view angle $45-90^{\circ}$; vertical anglo $10-15^{\circ}$; pull over available; larger viewing angles with no pull over

D. Horizontal view anglo $30-45^{\circ}$; no pull over available

B. Horizontal view angle $<30^{\circ}$; no pull over

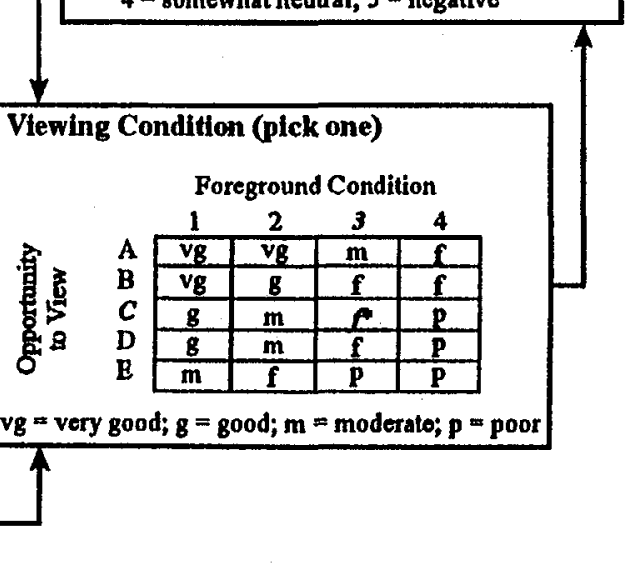




\section{Aesthetic Experience Worksheet Foothills Parkway Extension 8-B *** Views From the Parkway Extension *** \\ View '2', West up Webb Creek Valley, 4-580 to 4-700}

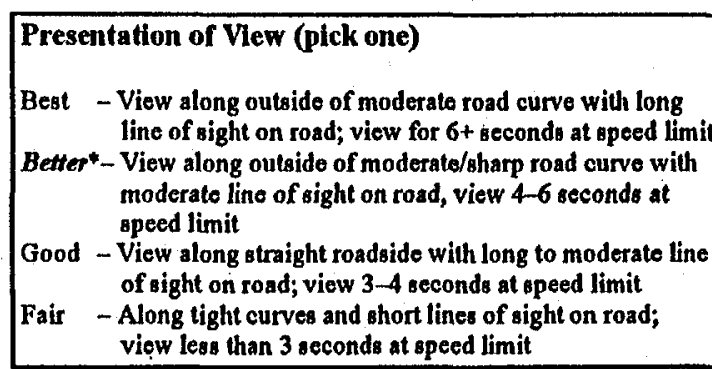

宗

\begin{tabular}{|l|}
\hline Spectal Experience Opportunity (pick one) \\
1. Fall color; spring blooms; water sounds; cool in summer, \\
very special lighting effects \\
II. Moming or evening back/side lighting; mists; winter \\
ice/frosts; deep woods; cool wet smells \\
III. Wildlife viewing (birds, deer, etc.); apecial geology; cool \\
woods smell; big trees; unusual vegetation; noticeablo \\
ecological processes \\
IV. Mostly normal mountain vegetation; little unusual lighting \\
effects or sensory experiences expected \\
V. Blinding sun; heavy fog; bad smella; bad traffic situation; \\
noisy; strong winds; dead and dying vegetation; hot in \\
aummer; trash in view
\end{tabular}

Choices are marked with an asteriak.

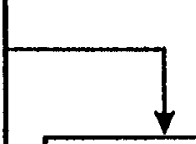
over present pull over present

\begin{tabular}{|c|c|}
\hline \multicolumn{2}{|c|}{ Quality of View (pick one) } \\
\hline \multicolumn{2}{|c|}{ Very Best - Water scenes with long views of series of } \\
\hline Best* & $\begin{array}{l}\text { - Long views of receding mountain ridges or } \\
\text { water scenes but not both; close views of } \\
\text { wooded mountain streams }\end{array}$ \\
\hline & $\begin{array}{l}\text { - Long but partially blocked views of mountain } \\
\text { ridges or water scenes; more midground ridges } \\
\text { than long viow }\end{array}$ \\
\hline & $\begin{array}{l}\text { - Midground viows of opposing ridges; rustic } \\
\text { valleys, little to no long views }\end{array}$ \\
\hline Fair & $\begin{array}{l}\text { - Midground to foreground viows of opposing } \\
\text { ridges, close views of roads, traffic; } \\
\text { dovelopment may be present }\end{array}$ \\
\hline
\end{tabular}
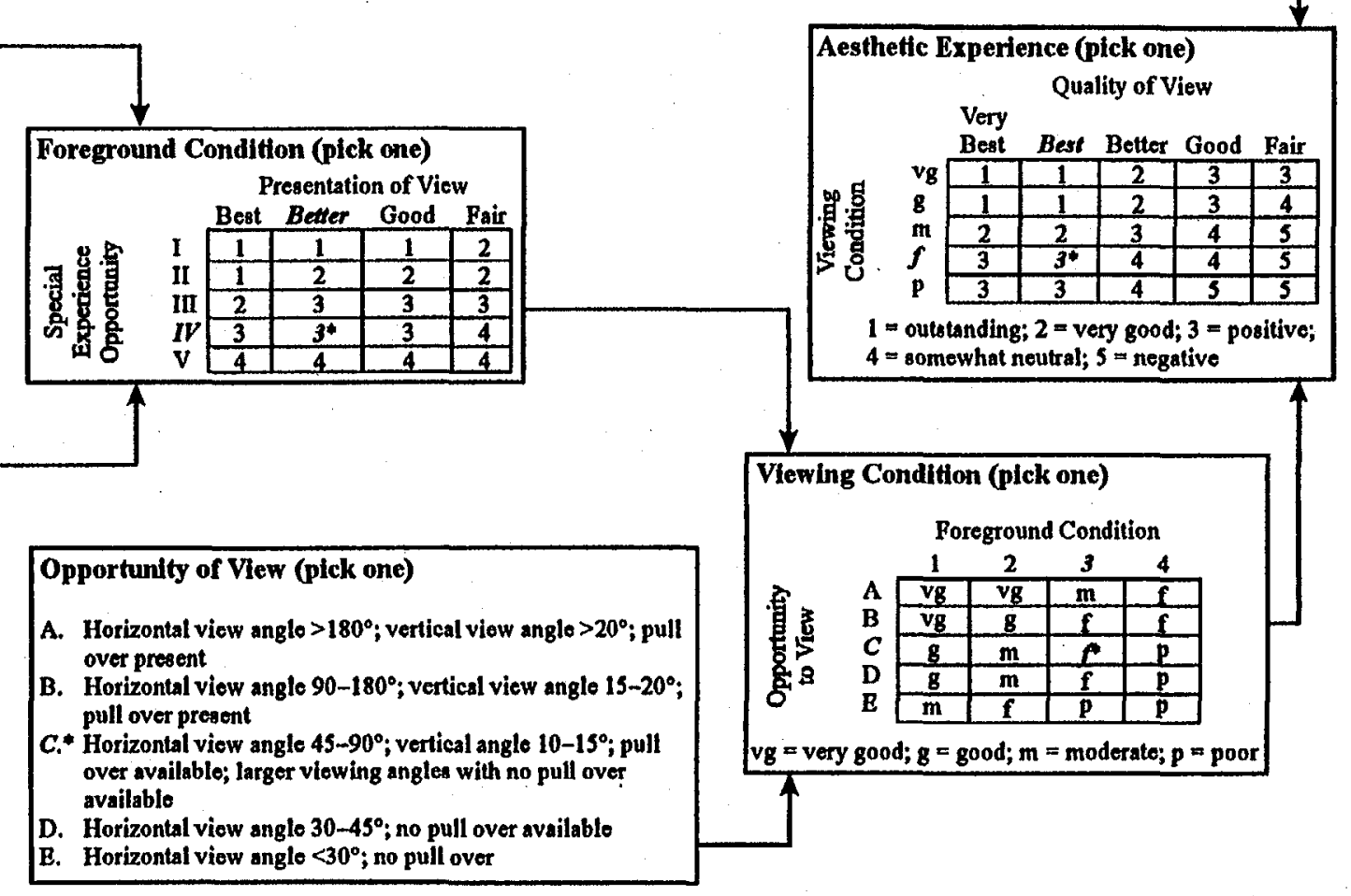

Opportunity of View (pick one)

A. Horizontal view anglo $>180^{\circ}$; vertical view angle $>20^{\circ}$; pull

B. Horizontal view angle $90-180^{\circ}$; vertical view angle $15-20^{\circ}$;

C.* Horizontal view angle $45-90^{\circ}$; vertical angle $10-15^{\circ}$; pull over available; larger viewing angles with no pull over

D. Horizontal view angle $30-45^{\circ}$; no pull over available

D. Horizontal viow anglo $<30^{\circ}$; no pull over 
Aesthetic Experience Worksheet

Foothills Parkway Extension 8-B

***. Views From the Parkway Extension $* * *$

\section{View '3', Timothy Creek, 4-940 to 5-200}

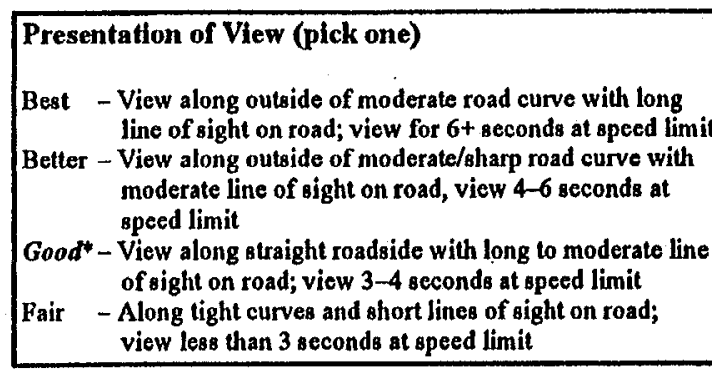

Special Experience Opportunity (pick one)

I. Fall color, spring blooms; water sounds; cool in summer, very special lighting effects

II.* Morning or evening back/side lighting; mista; winter ice/frosts; deep woods; cool wet smells

III. Wildlife viewing (birds, deer, etc.); tpecial geology; cool woods smell; big trees; unusual vegetation; noticeablo ecological processes

IV. Mostly normal mountain vegetation; little unusual lighting effects or sensory experiences expected

Blinding sun; hoavy fog; bad omells; bad traffic situation noisy; strong winds; dead and dying vegetation; hot in oummer; trash in view

Choices are marked with an anterisk

\begin{tabular}{|c|c|}
\hline \multicolumn{2}{|c|}{ Quality of View (pick one) } \\
\hline \multicolumn{2}{|c|}{ Very Best - Water scenes with long views of series of } \\
\hline \multicolumn{2}{|c|}{$\begin{array}{l}\text { Best } \quad \text { - Long views of receding mountain ridges or } \\
\text { water scenes but not both; close views of }\end{array}$} \\
\hline Better & $\begin{array}{l}\text { - Long but partially blocked viows of mountain } \\
\text { ridges or water scenes; more midground ridges } \\
\text { than long viow }\end{array}$ \\
\hline & $\begin{array}{l}\text { - Midground views of opposing ridges; rustic } \\
\text { valloys, little to no long viows }\end{array}$ \\
\hline Fair & $\begin{array}{l}\text { - Midground to foreground views of opposing } \\
\text { ridges, close views of roads, traffic; } \\
\text { development may be present }\end{array}$ \\
\hline
\end{tabular}

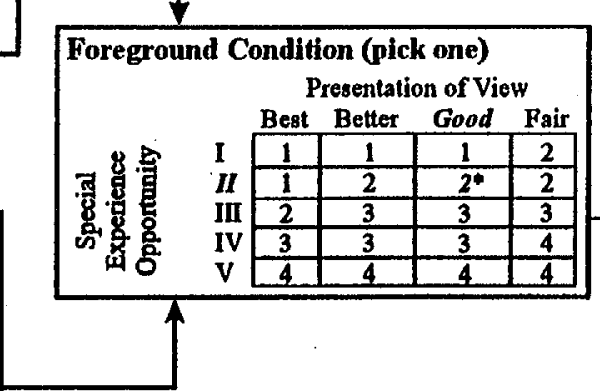

Opportunity of Vlew (pick one)

A. Horizontal view angle $>180^{\circ}$; vertical view angle $>20^{\circ}$; pull over present

B. Horizontal view angle $90-180^{\circ}$; vertical view angle $15-20^{\circ}$; pull over present

c.* Horizontal view angle $45-90^{\circ}$; vertical anglo $10-15^{\circ}$; pull over available; larger viewing angles with no pull over available

D. Horizontal view angle $30-45^{\circ}$; no pull over available

E. Horizontal view angle $\left\langle 30^{\circ}\right.$; no pull over
Aesthetic Experience (pick one)

Very

Best Best Better Good Fair

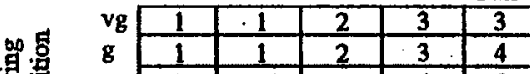

要

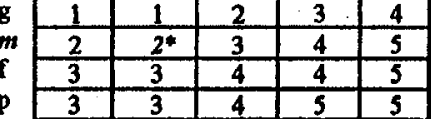

Putsonding; $2=$ very good; $3=$ posilive $4=$ somewhat neutral; $5=$ negativ

\section{casome}

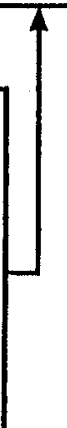

$v g=$ very good; $g=$ good; $m=$ moderate; $p=$ poor

Viewing Condition (pick one)

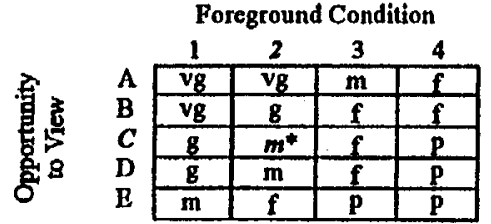




\section{Aesthetic Experience Worksheet Foothills Parkway Extension 8-B *** Views From the Parkway Extension ***}

\section{View 'E, SW Valley View, 6-000}

Special Experience Opportunity (pick one)

Fall color, spring blooms; water sounds; cool in summer very special lighting effects

Morning or evening back/side lighting; miats; winter ice/frosta; deep woods; cool wet smells

III. Wildlife viewing (birds, deer, etc.); special geology; cool woods smell; big trees; unusual vegetation; noticeable ecological processes

IV.* Mostly normal mountain vegetation; little unusual lighting effects or sensory experiences expected

V Blinding ann; heavy fog; bad smells; bad traffic situation Being; summer, trash in view

Choices are marked with an asterisk.

\begin{tabular}{|c|c|}
\hline \multicolumn{2}{|c|}{ Quality of View (pick one) } \\
\hline \multicolumn{2}{|c|}{$\begin{array}{l}\text { Very Best - Water scones with long views of series of } \\
\text { receding mountain ridges and valloys }\end{array}$} \\
\hline Best & $\begin{array}{l}\text { - Long viows of receding mountain ridges or } \\
\text { water scenes but not both; close views of } \\
\text { wooded mountain streams }\end{array}$ \\
\hline Better & $\begin{array}{l}\text { - Long but partially blocked views of mountain } \\
\text { ridges or water scenes; more midground ridges } \\
\text { than long view }\end{array}$ \\
\hline & $\begin{array}{l}\text { - Midground views of opposing ridges; rustic } \\
\text { valleys, little to no long views }\end{array}$ \\
\hline Fair* & $\begin{array}{l}\text { - Midground to foreground viows of opposing } \\
\text { ridges, close views of roads, traffic; } \\
\text { development may be present }\end{array}$ \\
\hline
\end{tabular}

$\downarrow$

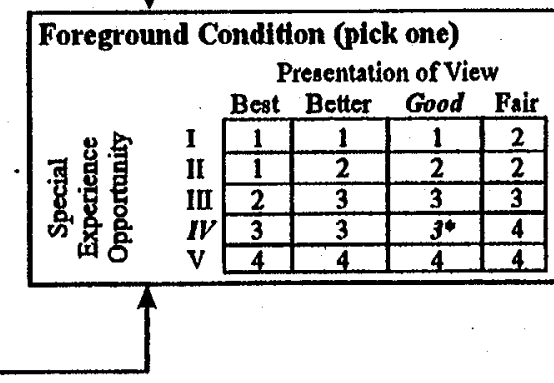
Aesthetic Experience (pick one)
Quality of View

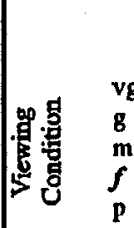

Best Best Better Good Fair

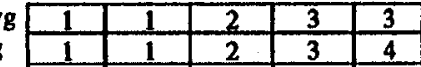

2

\begin{tabular}{|l|l|l|l|l|}
\hline 2 & 2 & 3 & 3 & 4 \\
\hline 3
\end{tabular}

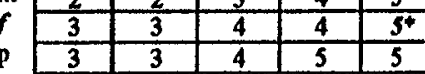

= oulatanding $2=$ very good $3=$ positive $4=$ somewhat neutral; $5=$ negative

\section{Opportunity of View (pick one)}

A. Horizontal view angle $>180^{\circ}$; vertical view angle $>20^{\circ}$; pull over present

B. Horizontal view angle $90-180^{\circ}$; vertical view angle $15-20^{\circ}$. pull over present

C. Horizontal view angle $45-90^{\circ}$; vertical angle $10-15^{\circ}$; pull over available; larger viewing angles with no pull over over availeble

D.* Horizontal view angle $30-45^{\circ}$; no pull over available

E. Horizontal view anglo $\left\langle 30^{\circ}\right.$; no pull over

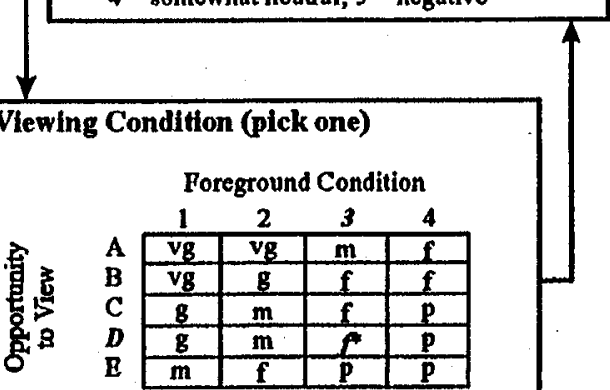

$\mathrm{vg}=$ very good; $\mathrm{g}=$ good; $\mathrm{m}=$ moderate; $\mathrm{p}=$ poor 


\section{Aesthetic Experience Worksheet Foothills Parkway Extension 8-B *** Views From the Parkway Extension ***}

\section{View '4', Lower Mill Dam Branch, 6-300 to 6-400}

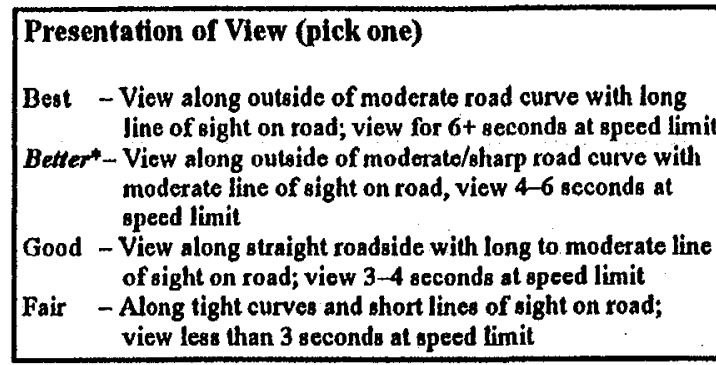

客

\begin{tabular}{|l|}
\hline Special Experience Opportunity (pick one) \\
I. \\
Fall color; spring blooms; water sounds; cool in summer, \\
very special lighting effects \\
II.* Morning or evening back/side lighting; mists; winter \\
ice/frosts; deop woods; cool wet smells \\
III. Wildlife viewing (birds, deer, etc.); special geology; cool \\
woods smell; big trees; unusual vegetation; noticeable \\
ecological processes \\
IV. Mostly normal mountain vegetation; little unusual lighting \\
effects or sensory experiences expected \\
V. Blinding sun; heavy fog; bad smells; bad traffic situation; \\
noisy; strong winds; doad and dying vegetation; hot in \\
summer; trash in view
\end{tabular}

Choices are marked with an asterisk.

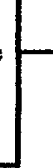
over present pull over present available

\begin{tabular}{|c|c|}
\hline \multicolumn{2}{|c|}{ Quality of View (pick one) } \\
\hline \multicolumn{2}{|c|}{$\begin{array}{c}\text { Very Best - Water scenes with long views of series of } \\
\text { receding mountain ridges and valleys }\end{array}$} \\
\hline Best* & $\begin{array}{l}\text { - Long viows of receding mountain ridges or } \\
\text { water scenes but not both; close viows of } \\
\text { wooded mountain streams }\end{array}$ \\
\hline Better & $\begin{array}{l}\text { - Long but partially blocked views of mountain } \\
\text { ridges or water scenes; more midground ridges } \\
\text { than long view }\end{array}$ \\
\hline & $\begin{array}{l}\text { - Midground views of opposing ridges; rustic } \\
\text { valloys, little to no long views }\end{array}$ \\
\hline Fair & $\begin{array}{l}\text { - Midground to foreground viows of opposing } \\
\text { ridges, close views of roads, traffio; } \\
\text { development may be present }\end{array}$ \\
\hline
\end{tabular}

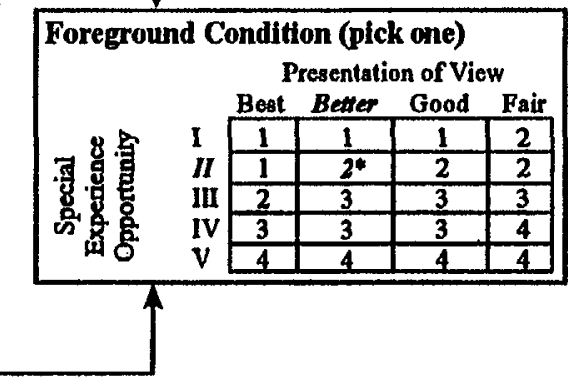

Aesthetic Experience (pick one)
Quality of View Very

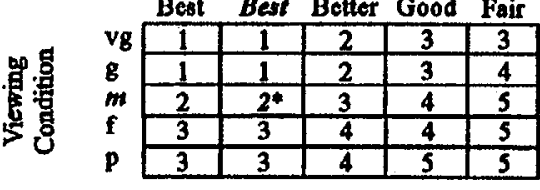

1 = outatanding; 2 = very good; 3 = positive; $4=$ somewhat neutral; $5=$ negative

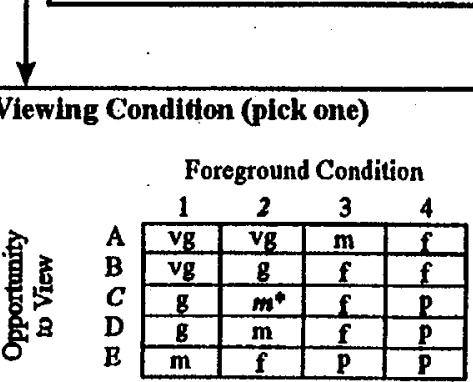

Opportunity of View (pick one)

A. Horizontal view angle $>180^{\circ}$; vertical view angle $>20^{\circ}$; pull

B. Horizontal view angle $90-180^{\circ}$; vertical view angle $15-20^{\circ}$;

C. Horizontal view anglo $45-90^{\circ}$; vertical anglo $10-15^{\circ}$; pull over available; larger viewing angles with no pull over

D. Horizontal view angle $30-45^{\circ}$; no pull over available

B. Horizontal view anglo $\left\langle 30^{\circ}\right.$; no pull over $v g=$ very good; $g=$ good; $m=$ moderate; $p=$ poor 


\section{Aesthetic Experience Worksheet Foothills Parkway Extension 8-B *** Views From the Parkway Extension ***}

\section{View 'F, Webb Mountain Top, Parking Lot}

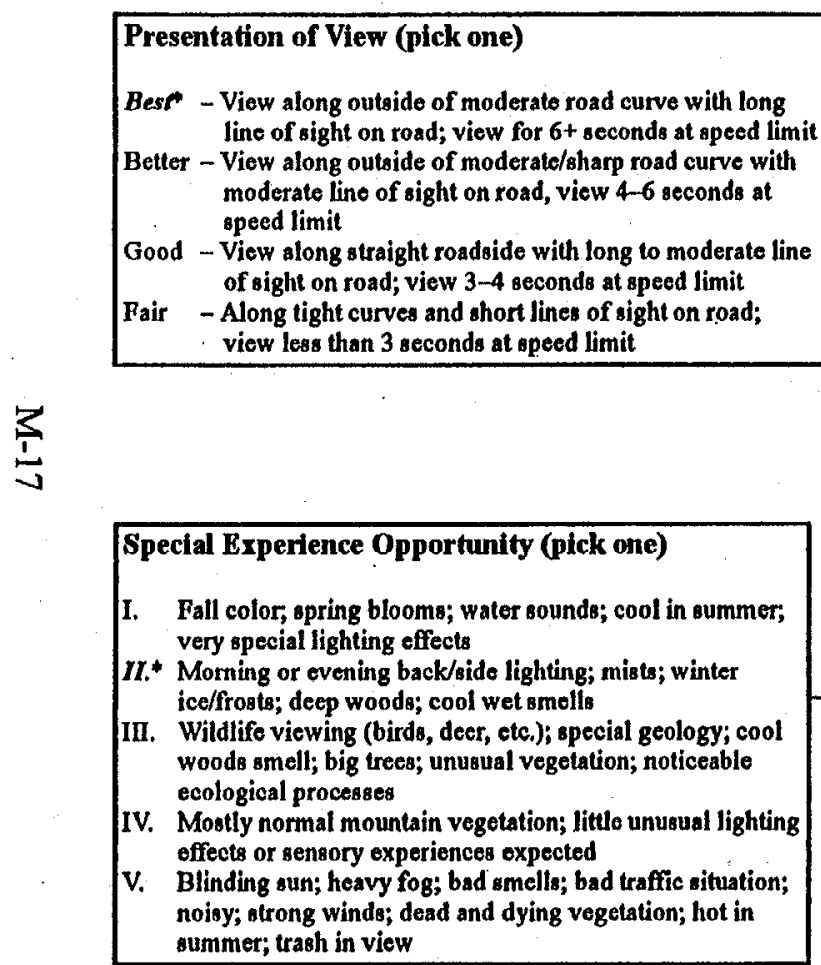

Choices are marked with an asterisk. over present available

\begin{tabular}{|c|c|}
\hline \multicolumn{2}{|c|}{ Quality of View (pick one) } \\
\hline \multicolumn{2}{|c|}{$\begin{array}{l}\text { Very Best - Water scenes with long views of series of } \\
\text { receding mountain ridges and valleys }\end{array}$} \\
\hline Best* & $\begin{array}{l}\text { - Long views of receding mountain ridges or } \\
\text { water scenes but not both; close views of } \\
\text { wooded mountain streams }\end{array}$ \\
\hline Better & $\begin{array}{l}\text { - Long but partially blocked views of mountain } \\
\text { ridges or water scenes; more midground ridges } \\
\text { than long view }\end{array}$ \\
\hline & - Midground views of opposing ridges; nustic \\
\hline Fair & $\begin{array}{l}\text { - Midground to foreground views of opposing } \\
\text { ridges, close views of roads, traffic; } \\
\text { development may be present }\end{array}$ \\
\hline
\end{tabular}

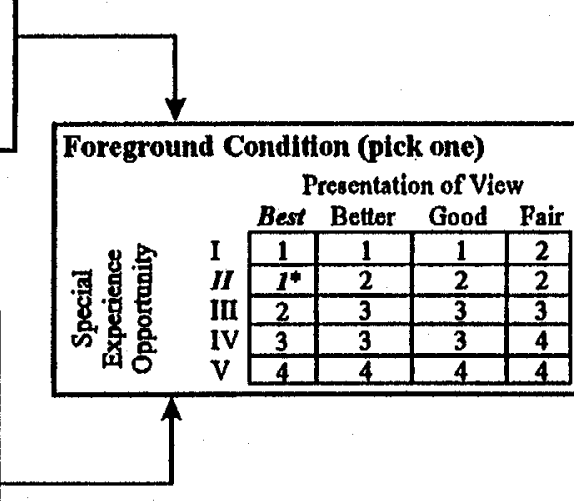

Opportunity of View (pick one)

A." Horizontal view angle $>180^{\circ}$; vertical view angle $>20^{\circ}$; pull

B. Horizontal view angle $90-180^{\circ}$; vertical view angle $15-20^{\circ}$;

C. Horizontal view angle $45-90^{\circ}$; vertical angle $10-15^{\circ}$; pull over availablo; larger viewing angles with no pull over

D. Horizontal view angle $30-45^{\circ}$; no pull over available

B. Horizontal view angle $<30^{\circ}$; no pull over
Aesthetic Experience (pick one) Quality of View

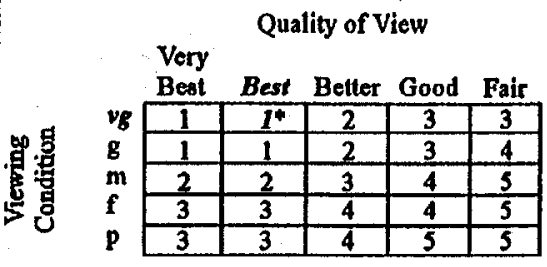

1 = outstanding; 2 = very good; 3 = positive; $4=$ somewhat neutral; $5=$ negative

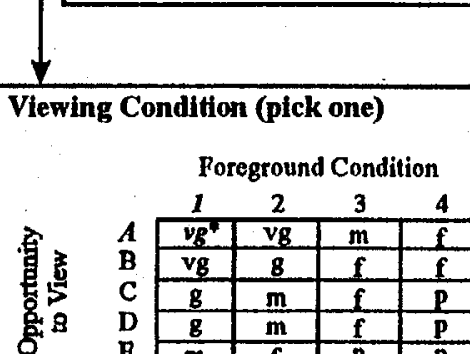

$v g=$ very good; $g=$ good; $m$ m moderate; $p=$ poor 
Aesthetic Experience Worksheet

Foothills Parkway Extension 8-B

*** Views From the Parkway Extension ***

\section{View '5', Warden Branch; 7-810}

Special Experience Opportunity (pick one)

I. Fall color, spring bloom; water sounds; cool in summer very special lighting effects

II. Morning or evening back/side lighting; mists; winter ice/frosis; deep woods; cool wet smells

III. Wildfife viewing (birds, deer, etc.); special geology; cool woods omeil; big trees; unusual vegetation; noticeable ecological processes

IV. * Mostly normal mountain vegetation; little unusual lighting effects or sensory experiences expected

Blinding sun; heavy fog; bad smello; bad traffic situation; noisy; strong winds; dead and dying vegetation; hot in summer; trash in viow

Choices are marked with an asterisk.

\begin{tabular}{|c|c|}
\hline \multicolumn{2}{|c|}{ Quallty of View (pick one) } \\
\hline \multicolumn{2}{|c|}{$\begin{array}{c}\text { Very Best - Water scenes with long views of series of } \\
\text { receding mountain ridges and valleys }\end{array}$} \\
\hline Best* & $\begin{array}{l}\text { - Long views of receding mountain ridges or } \\
\text { water scenes but not both; close views of } \\
\text { wooded mountain streams }\end{array}$ \\
\hline Better & $\begin{array}{l}\text { - Long but partially blocked views of mountain } \\
\text { ridges or water scenes; more midground ridge } \\
\text { than long view }\end{array}$ \\
\hline & $\begin{array}{l}\text { - Midground views of opposing ridges; rustic } \\
\text { valleys, little to no long views }\end{array}$ \\
\hline Fair & $\begin{array}{l}\text { - Midground to foreground views of opposing } \\
\text { ridges, close views of roads, traffic; } \\
\text { dovelopment may be present }\end{array}$ \\
\hline
\end{tabular}
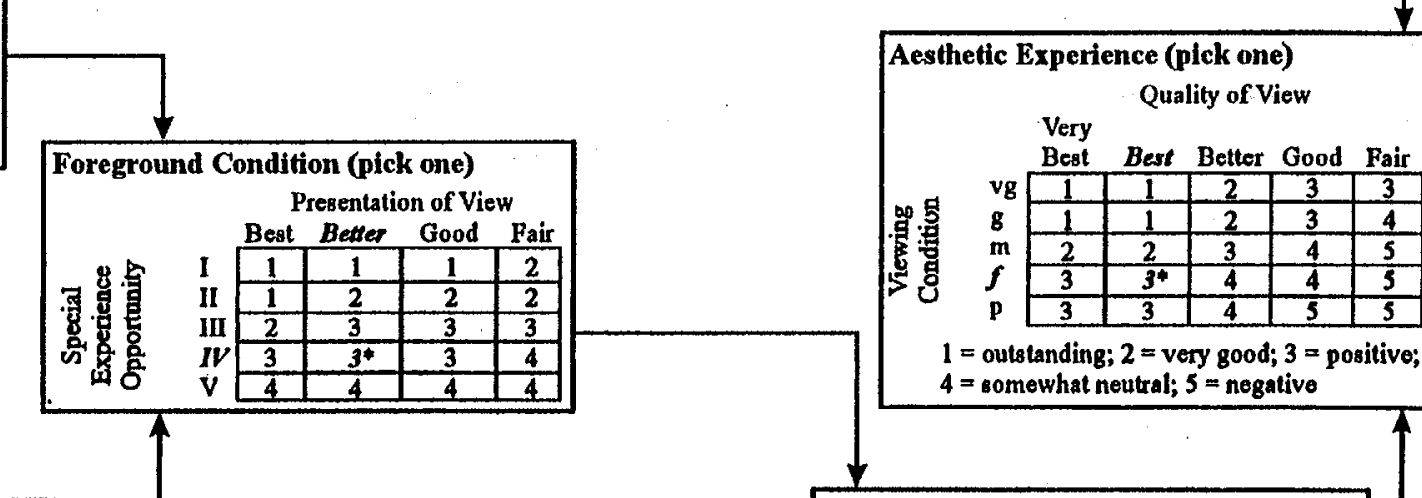

1 = oulstanding; 2 = very good; 3 = positive; $4=$ somewhat neutral; $5=$ negative

\section{Opportunity of View (pick one)}

A. Horizontal view angle $>180^{\circ}$; vertical view angle $>20^{\circ}$; pult over present

B. Horizontal view angle $90-180^{\circ}$; vertical view angle $15-20^{\circ}$;

pull over present

C. Horizontal view angle $45-90^{\circ}$; vertical angle $10-15^{\circ}$; pull over available; larger viewing angles with no pull over available

D.* Horizontal view angle $30-45^{\circ}$; no pull over availablo

B. Horizontal view angle $<30^{\circ}$; no pull over

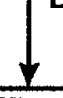

Viewing Condition (pick one)

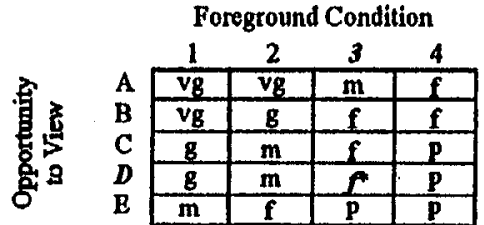

$\mathrm{vg}=$ very good; $g=$ good; $m=$ moderate; $p=$ poor 


\section{Aesthetic Experience Worksheet Foothills Parkway Extension 8-B *** Views From the Parkway Extension ***}

\section{View '6', Upper Warden Branch, 8-120 to 9-170}

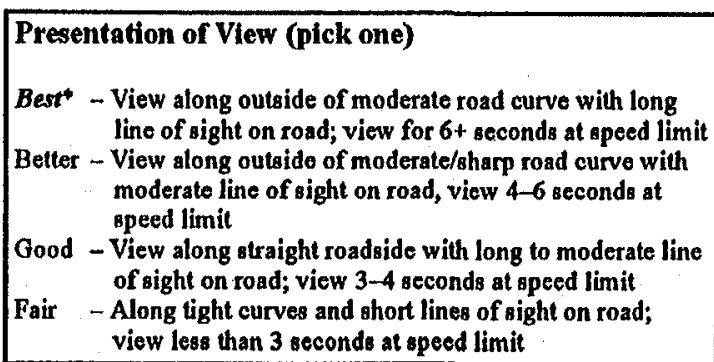

\section{Special Experience Opportunity (pick one)}

I.* Fall color, spring blooms; water sounds; cool in summer very special lighting effects

Moming or evening back/side lighting; mists; winter ice/frosts; deep woods; cool wet smells

III. Wildlife viewing (birds, deer, etc.); special geology; cool woods smell; big trees; unusual vegetation; noticeable ecological processes

IV. Mostly nommal mountain vegetation; little unusual lighting effects or sensory experiences expected

Blinding sun; heavy fog; bad smells; bad traffic situation; noisy; strong winda; dead and dying vegetation; hot in summer, trash in view

Choices are marked with an asterisk

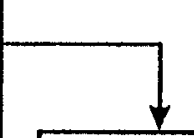
ver present

pull over present availablo

\begin{tabular}{|c|c|}
\hline Qualit & of View (pick one) \\
\hline & $\begin{array}{l}t \text { - Water scenes with long views of series of } \\
\text { receding mountain ridges and valleys }\end{array}$ \\
\hline Best* & $\begin{array}{l}\text { - Long views of receding mountain ridges or } \\
\text { water scenes but not both; close views of } \\
\text { wooded mountain streams }\end{array}$ \\
\hline Better & $\begin{array}{l}\text { - Long but partially blocked viows of mountain } \\
\text { ridges or water scenes; more midground ridges } \\
\text { than long view }\end{array}$ \\
\hline Good & $\begin{array}{l}\text { - Midground views of opposing ridges; rustic } \\
\text { valleys, little to no long views }\end{array}$ \\
\hline Fair & $\begin{array}{l}\text { - Midground to foreground views of opposing } \\
\text { ridges, close views of roads, traffic; } \\
\text { development may be present }\end{array}$ \\
\hline
\end{tabular}

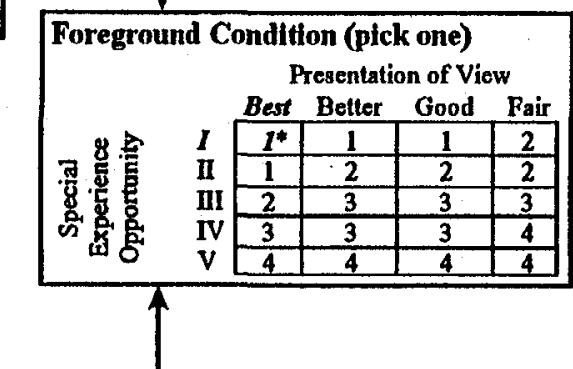

Aesthetic Experience (pick one)
Quality of View

Very

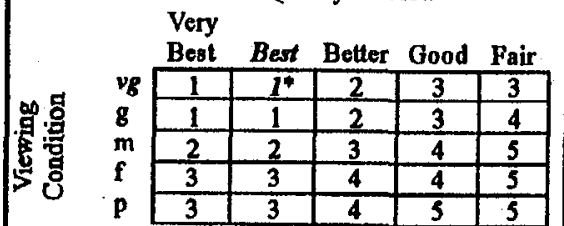

$1=$ outstanding; 2 = very good; 3 = positive; $4=$ somewhat neutral; $5=$ negative

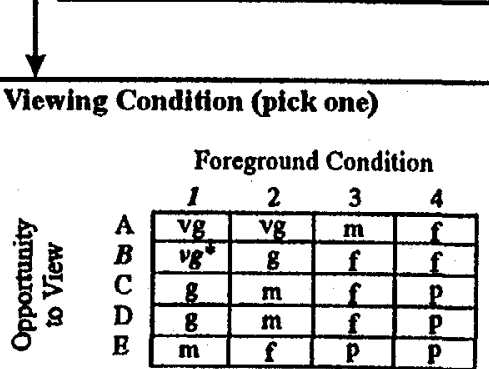

izontal view angle $>180^{\circ}$; vertical view angle $>20^{\circ}$; pull

* Horizontal view angle $90-180^{\circ}$; vertical vicw angle $15-20^{\circ}$ i

Horizontal view angle $45-90^{\circ}$; vertical angle $10-15^{\circ}$; pull over available; larger viewing angles with no pull over

D. Horizontal view angle $30-45^{\circ}$; no pull over available

B. Horizontal view angle $\left\langle 30^{\circ}\right.$; no pull over 


\section{Aesthetic Experience Worksheet Foothills Parkway Extension 8-B *** Views From the Parkway Extension *** \\ View '7', Jones Gap N, Webb Mtn. Parking Access Rd.}

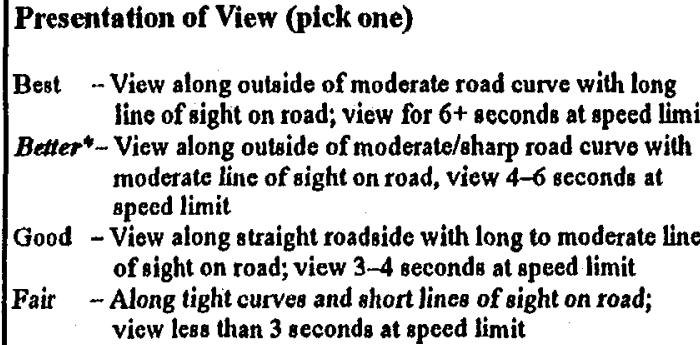
view less than 3 seconds at speed limit

恧

\section{Special Experience Opportunity (pick one)}

Fall color; apring blooms; water sounds; cool in summer; very special lighting effects

* Morning or evening back/side lighting; mists; winter ice/frosts; deep wooda; cool wel smells

III. Wildlife viewing (birds, deer, etc.); special geology; cool woods amell; big trees; unusual vegetation; noticeable ecological processes

IV. Mostly normal mountain vegetation; little unusual lighting effects or sensory experiences expected

V. Blinding sun; heavy fog; bad smells; bad traffic situation; noisy; strong winds; dead and dying vegetation; hot in summer; trash in view

Choices are marked with an asterisk.

\begin{tabular}{|c|c|}
\hline \multicolumn{2}{|c|}{ Quality of View (pick one) } \\
\hline & - Water scenes with long views of series of \\
\hline Best* & $\begin{array}{l}\text { - Long views of receding mountain ridges or } \\
\text { water scenes but not both; close views of } \\
\text { wooded mountain streams }\end{array}$ \\
\hline Better & $\begin{array}{l}\text { - Long but partially blocked views of mountain } \\
\text { ridges or water scenes; more midground ridges } \\
\text { than lone view }\end{array}$ \\
\hline & $\begin{array}{l}\text { - Midground views of opposing ridges; rustio } \\
\text { valleys, Jittle to no long viows }\end{array}$ \\
\hline Fair & $\begin{array}{l}\text { - Midground to foreground views of opposing } \\
\text { ridges, close viows of roads, traffic; } \\
\text { development may be present }\end{array}$ \\
\hline
\end{tabular}

$\downarrow$

Foreground Condition (pick one) Presentation of View

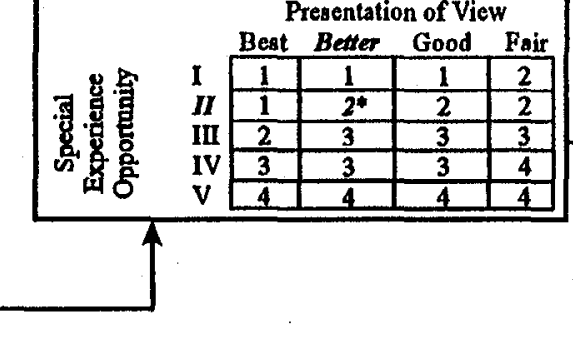

Opportunity of View (pick one)

A. Horizontal view angle $>180^{\circ}$; vertical view angle $>20^{\circ}$; pull over present

B. Horizontal view angle $90-180^{\circ}$; vertical view angle 15-20 pull over present

C.* Horizontal view angle $45-90^{\circ}$; vertical angle $10-15^{\circ}$; pull over available; larger viewing anglea with no pull over available

D. Horizontal view angle $30-45^{\circ}$; no pull over available B. Horizontal view angle $<0^{\circ}$; no pull over

\section{Aesthetic Experience (pick one)}

Quality of View

Very

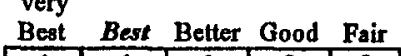

\begin{tabular}{ll|l|l|l|l|l|} 
& vg & 1 & 1 & 2 & 3 & 3 \\
\hline 1 & 1 & 2 & 3 & 4 \\
\hline
\end{tabular}

5 ở

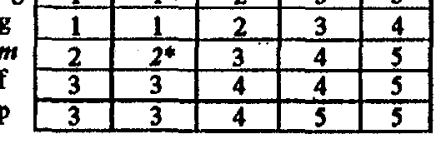

= outstanding; 2 = very good; 3 = positive; $4=$ somewhal neutral; $5=$ negative

$\downarrow$

Viewing Condition (pick one)

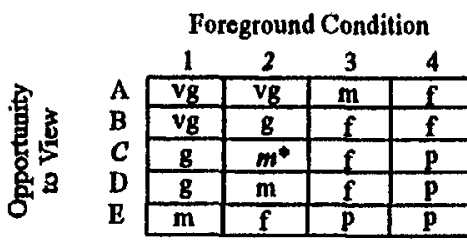

$\mathrm{vg}=$ very good; $g=$ good; $m=$ moderate; $p=$ poor 


\section{Aesthetic Experience Worksheet Foothills Parkway Extension 8-B *** Views From the Parkway Extension ***}

\section{View 'G', Black Gum Gap S, 10-450}

交

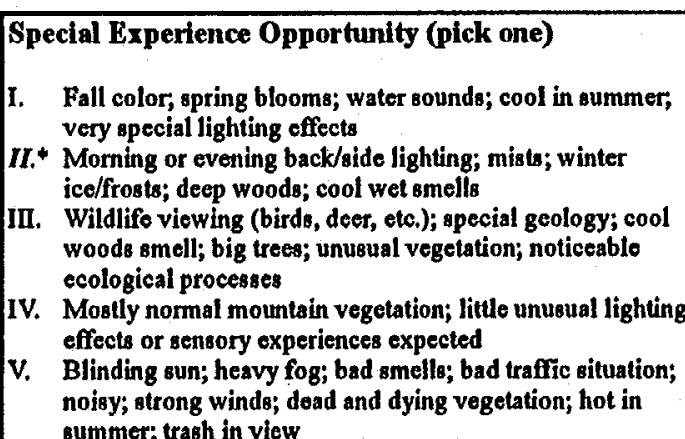
summer; trash in view

Choices are marked with an asterisk.

\begin{tabular}{|c|c|}
\hline \multicolumn{2}{|c|}{ Quality of View (pick one) } \\
\hline \multicolumn{2}{|c|}{ Very Best - Water scenes with long views of series of } \\
\hline & $\begin{array}{l}\text { Long viows of receding mountain ridges or } \\
\text { water scenes but not both; close views of } \\
\text { wooded mountain streams }\end{array}$ \\
\hline Better* & $\begin{array}{l}\text { - Long but partially blocked views of mountain } \\
\text { ridges or water scenes; more midground ridges } \\
\text { than long view }\end{array}$ \\
\hline & $\begin{array}{l}\text { - Midground views of opposing ridges; rustic } \\
\text { valleys, little to no long views }\end{array}$ \\
\hline Fair & $\begin{array}{l}\text { - Midground to foreground views of opposing } \\
\text { ridges, olose views of roads, traffic; } \\
\text { development may be present }\end{array}$ \\
\hline
\end{tabular}

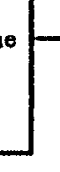
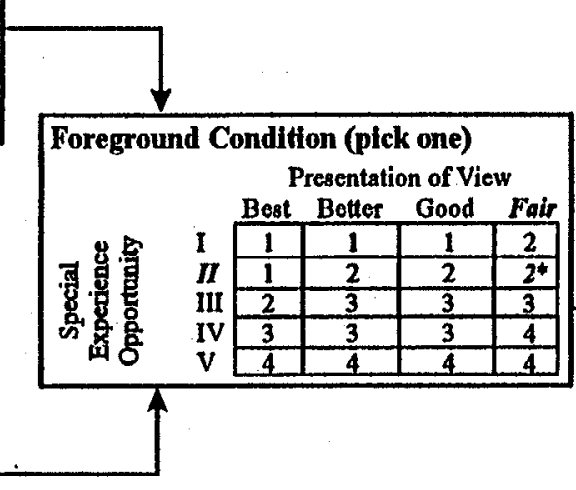

\section{Opporfunity of View (pick one)}

A. Horizontal view angle $>180^{\circ}$; vertical view angle $>20^{\circ}$; pull over present

B. Horizontal view angle $90-180^{\circ}$; vertical view angle $15-20^{\circ}$; pull over present

C. Horizontal view anglo $45-90^{\circ}$; vertical anglo $10-15^{\circ}$; pull over available; larger viowing angles with no pull over available

D.* Horizontal view angle $30-45^{\circ}$; no pull over available

B. Horizontal view angle $<30^{\circ}$; no pull over
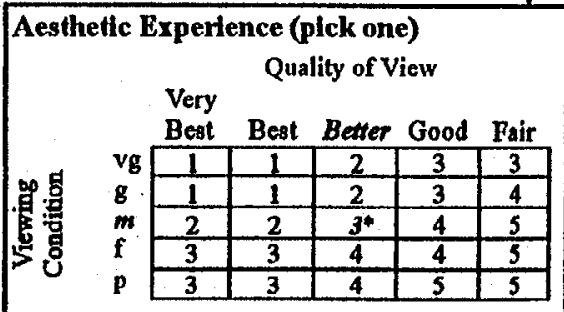

$=$ outstanding; $2=$ very good; $3=$ positive; $4=$ somewhat neutral; $5=$ negative

$\downarrow$

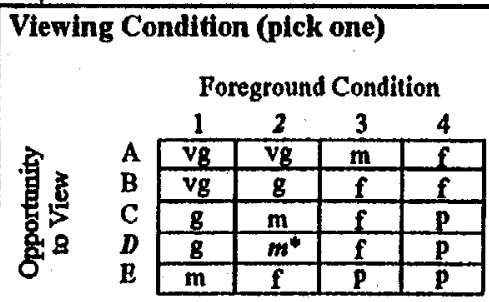

$v g=$ very good; $g=$ good; $m=$ moderate $p=$ poor 


\section{Aesthetic Experience Worksheet Foothills Parkway Extension 8-B *** Views From the Parkway Extension ***}

\section{View 'H', Black Gum Gap N, 10-450}

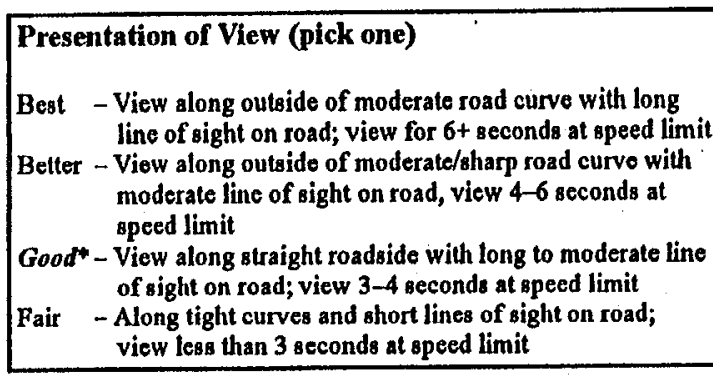

\begin{tabular}{|l|}
\hline Special Experience Opportunity (pick one) \\
I. \\
Fall color; apring blooms; water sounds; cool in summer; \\
very special lighting effects \\
II. Morning or evening back/side lighting; mists; winter \\
ice/frosts; deep woods; cool wet smells \\
III. Wildlifo viewing (birds, deer, etc.); special geology; cool \\
woods smell; big trees; unusual vegetation; noticeable \\
ecological processes \\
IV.* Mostly normal mountain vegetation; little unusual lighting \\
effects or sensory experiences expected \\
V. Blinding sun; heavy fog; bad smells; bad traffic situation; \\
noisy; strong winds; dead and dying vegetation; hot in \\
summer; trash in view
\end{tabular}

Choices are marked with an asterisk.

\begin{tabular}{|c|c|}
\hline Qualit & of View (pick one) \\
\hline Very $\mathrm{Be}$ & - Water scenes with long views of series of \\
\hline Best & $\begin{array}{l}\text { - Long viows of receding mountain ridges or } \\
\text { water scenes but not both; close viows of } \\
\text { wooded mountain streams }\end{array}$ \\
\hline Bettor & $\begin{array}{l}\text { - Long but partially blocked views of mountain } \\
\text { ridges or water scenes; more midground ridges } \\
\text { than long view }\end{array}$ \\
\hline & $\begin{array}{l}\text { - Midground views of opposing ridges; rustic } \\
\text { valleys, little to no long views }\end{array}$ \\
\hline Fair & $\begin{array}{l}\text { - Midground to foreground views of opposing } \\
\text { ridges, close views of roads, traffic; } \\
\text { development may be present }\end{array}$ \\
\hline
\end{tabular}

\section{$\downarrow$}

Foreground Condition (pick one)

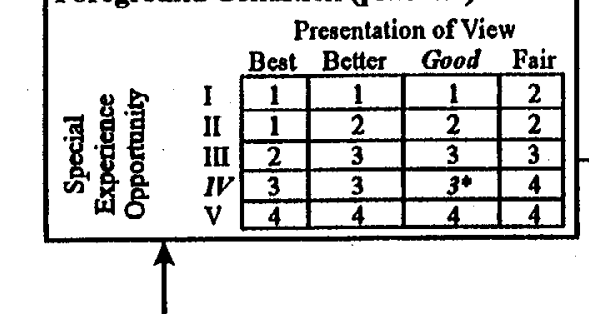

A. Horizontal view anglo $>180^{\circ}$; vertical view angle $>20^{\circ}$; pull over present

B. Horizontal view angle $90-180^{\circ}$; vertical view angle $15-20^{\circ}$; pull over present

C. Horizontal view angle $45-90^{\circ}$; vertical angle $10-15^{\circ}$; pull over available; larger viewing angles with no pull over available

D. Horizontal view angle $30-45^{\circ}$; no pull over available

B. Horizontal view angle $<30^{\circ}$; no pull over Presentation of View

\section{Opportunity of Vlew (pick one)}

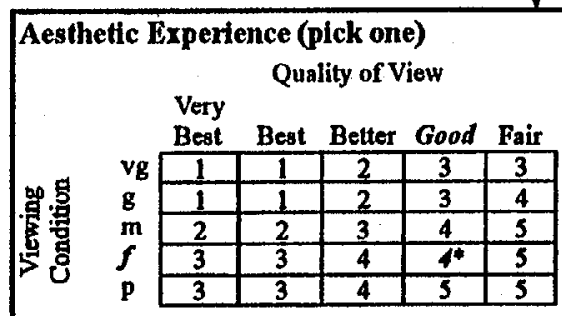

1 = outstanding; 2 = very good; 3 = positivo; $4=$ somewhat neutral; $5=$ negative

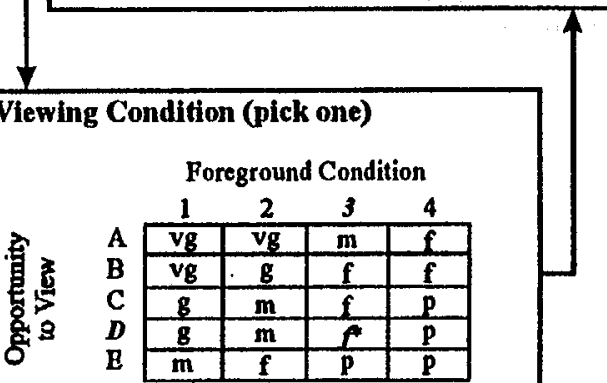

$v g=$ very good; $g=$ good; $m$ = moderate; $p=$ poor 


\section{Aesthetic Experience Worksheet Foothills Parkway Extension 8-B *** Views From the Parkway Extension ***}

\section{View '8', Table Ridge, 11-500 to 11-950}

\author{
Presentation of View (pick one) \\ Besc - View along outside of moderate road curve with long \\ line of sight on road; view for $6+$ seconds at apeed limit \\ Better - View along outside of moderate/sharp road curve with \\ moderate line of sight on road, view 4-6 seconds at \\ speed limit \\ Good - View along straight roadside with long to moderate line \\ of sight on road; view 3-4 seconds at speed limit \\ Tair - Along tight curves and short lines of sight on road. \\ view less than 3 seconds at speed limit
}

\section{Special Experience Opportunity (pick one)}

I. Fall color, spring blooms; water sounds; cool in summer very special lighting offects

II. Morning or evening back/side lighting; mists; winter ice/frosts; deep woods; cool wet smells

III.* Wildlife viewing (birds, deer, etc.); special geology; cool woods amell; big trees; unusual vegetation; noticeablo ecological processes

IV. Mostly normal mountain vegetalion; little unusual lighting effects or sensory experiences expected

V. Blinding sun; heavy fog; bad amells; bad traffic aituation; noisy; strong winds; dead and dying vegetation; hot in oummer; trash in view

Choices are marked with an asterisk

$\square$
over present pull over present

\begin{tabular}{|c|c|}
\hline \multicolumn{2}{|c|}{ Quality of View (pick one) } \\
\hline \multicolumn{2}{|c|}{$\begin{array}{l}\text { Very Best - Water scenes with long views of series of } \\
\text { receding mountain ridges and valleys }\end{array}$} \\
\hline Best* & $\begin{array}{l}\text { - Long views of receding mountain ridges or } \\
\text { water scenes but not both; close views of } \\
\text { wooded mountain streams }\end{array}$ \\
\hline Better & $\begin{array}{l}\text { - Long but partially blocked views of mountain } \\
\text { ridges or water scenes; more midground ridges } \\
\text { than long view }\end{array}$ \\
\hline & $\begin{array}{l}\text { - Midground views of opposing ridges; rustic } \\
\text { valleys, little to no long views }\end{array}$ \\
\hline Fair & $\begin{array}{l}\text { - Midground to foreground views of opposing } \\
\text { ridges, close views of roads, traffic; } \\
\text { development may be present }\end{array}$ \\
\hline
\end{tabular}

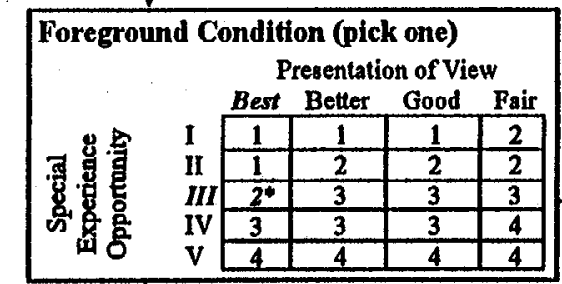

Aesthetic Experience (pick one)
Quality of View

Very

Best Best Better Good Fair

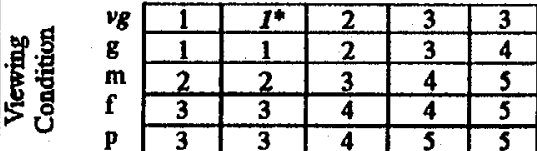

1 = outstanding; 2 = very good; 3 = positive; $4=$ somewhat neutral; $5=$ negative

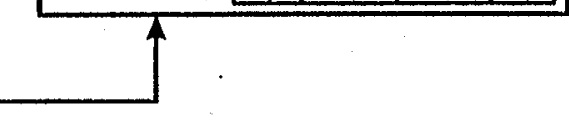

Opportunity of View (pick one)

A.* Horizontal view angle $>180^{\circ}$; vertical view angle $>20^{\circ}$; pull

B. Horizontal view angle $90-180^{\circ}$; vertical view anglo $15-20^{\circ}$;

C. Horizontal view angle $45-90^{\circ}$; vertical angle $10-15^{\circ}$; pull over availablo; larger viewing angles with no pull over

D. Horizontal view angle $30-45^{\circ}$; no pull over availablo

B. Horizontal view angle $<30^{\circ}$; no pull over

Viewing Condition (pick one)

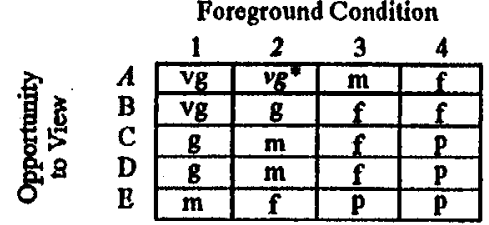

$v g=$ very good; $g=$ good; $m=$ moderate; $p=$ poor 
Aesthetic Experience Worksheet

Foothills Parkway Extension 8-B

*** Views From the Parkway Extension ***

\section{View '9', Branam Hollow, 12-300 to 12-410}

\section{Presentation of View (pick one)}

Best - View along outside of moderate road curve with long line of sight on road; vicw for $6+$ seconds at speed limit

Better - View along outside of moderate/sharp road curve with moderate line of sight on road, view 4-6 seconds at speed limit

Good - View along straight roadside with long to moderato line of sight on road; view 3-4 seconds at speed limit

Fair* - Along tight curves and short lines of sight on road; view less than 3 seconds at speed limi

\section{Special Experience Opportunity (pick one)}

I. Fall color, spring blooma; water sounds; cool in summer; very special lighting effects

II. Moming or evening back/side lighting; mists; winter ice/frosts; deep woods; cool wet smells

III.* Wildlife viewing (birds, deer, etc.); special geology; cool woods smell; big trees; unusual vegetation; noticeable ecological processes

IV. Mostly normal mountain vegetation; little unusual lighting effects or sensory experiences expected

V. Blinding oun; heavy fog; bad omello; bad traffic aituation; noisy; strong winds; dead and dying vegetation; hot in summer; trash in view

Choices are marked with an asterisk

\begin{tabular}{|c|c|}
\hline \\
\hline \multicolumn{2}{|c|}{$\begin{array}{l}\text { Very Best - Water scenes with long views of series of } \\
\text { receding mountain ridges and valleys }\end{array}$} \\
\hline \multicolumn{2}{|c|}{$\begin{array}{l}\text { Best - Long viows of receding mountain ridges or } \\
\text { water scenes but not both; close views of } \\
\text { wooded mountain streams }\end{array}$} \\
\hline \multicolumn{2}{|c|}{$\begin{array}{l}\text { wooded mountain streams } \\
\text { Better - Long but partially blocked views of mountain } \\
\text { ridges or water scenes; more midground ridges }\end{array}$} \\
\hline \multirow{2}{*}{$\begin{array}{l}\text { Good* } \\
\text { Fair }\end{array}$} & $\begin{array}{l}\text { - Midground views of opposing ridges; rustio } \\
\text { valleys, little to no long viows }\end{array}$ \\
\hline & $\begin{array}{l}\text { - Midground to foreground views of opposing } \\
\text { ridges, close views of roads, traffic; } \\
\text { development may be present }\end{array}$ \\
\hline
\end{tabular}

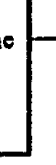

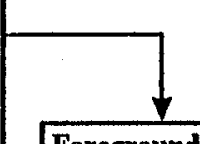

Foreground Condition (pick one) Presentation of View

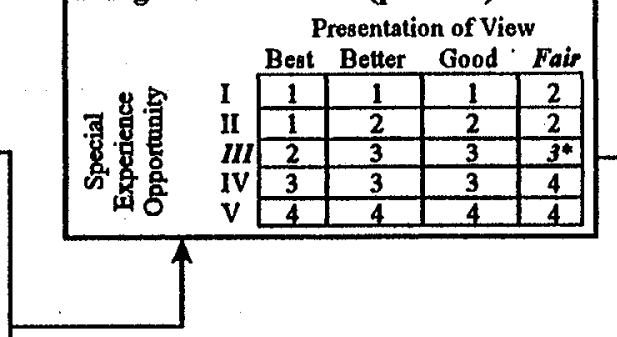

Opportunity of View (pick one)

A. Horizontal view angle $>180^{\circ}$; vertical viow angle $>20^{\circ}$; pull over present

B. Horizontal view angle $90-180^{\circ}$; vertical view anglo $15-20^{\circ}$; pull over present

C. Horizontal view angle $45-90^{\circ}$; vertical angle $10-15^{\circ}$; pull over available; larger viewing angles with no pull over over availablo
available

D.* Horizontal view angle $30-45^{\circ}$; no pull over available

B. Horizontal view angle $<30^{\circ}$; no pull over
Aesthetic Experience (pick one)

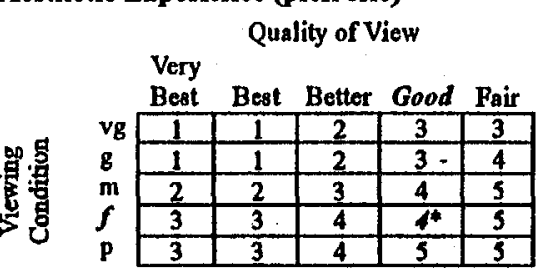

1 = outstanding; 2 = very good; 3 = positive; $4=$ somewhat neutral; $5=$ negative

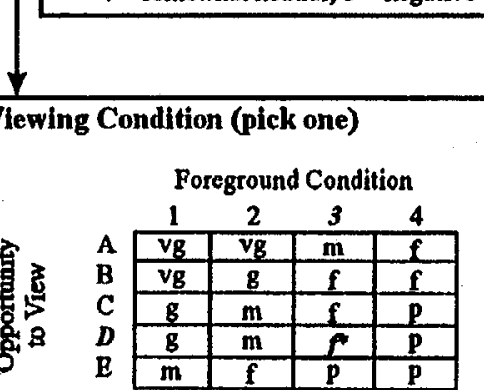

vg $\approx$ very good; $g=$ good; $m=$ moderate; $p=$ poor 
Aesthetic Experience Worksheet

Foothills Parkway Extension 8-B

*** Views From the Parkway Extension ***

\section{View '10', Pine Cove, 12-510}

\section{Presentation of View (pick one)}

Best - View along outside of moderate road curve with long line of sight on road; view for $6+$ seconds at speed limit Better - View along outside of moderate/sharp road curve with moderate line of sight on road, view $4-6$ seconds at speed limit

Good - View along straight roadside with long to moderate line of sight on road; view 3-4 seconds at speed limit

Fair* - Along tight curves and short lines of sight on road; viow less than 3 aeconds at speed limil

管.

\section{Special Experience Opportunity (pick one)}

1. Fall color, apring blooms; water sounda; cool in summer, very special lighting effects

II. Moming or evening back/aide lighting; mists; winte ice/frosts; deep woods; cool wet smells

III.* Wildlife viewing (birds, deer, etc.); special geology; cool woods smell; big trees; unusual vegetation; noticeable ecological processes

IV. Mostly normal mountain vegetation; littlo unusual lighting effects or sensory experiences expected

Blinding sun; heavy fog; bad smells; bad traffic situation: noiry; strong winds; dead and dying vegetation; hot in summer; trash in view

Choices are marked with an asterisk

\begin{tabular}{|c|c|}
\hline \multicolumn{2}{|c|}{ Quality of View (pick one) } \\
\hline \multicolumn{2}{|c|}{$\begin{array}{l}\text { Very Best - Water scenes with long views of series of } \\
\text { receding mountain ridges and valloys }\end{array}$} \\
\hline & $\begin{array}{l}\text { - Long viows of receding mountain ridges or } \\
\text { water scenes but not both; close views of } \\
\text { wooded mountain streams }\end{array}$ \\
\hline Better & $\begin{array}{l}\text { - Long but partially blocked views of mountain } \\
\text { ridges or water scenes; more midground ridges } \\
\text { than long view }\end{array}$ \\
\hline & $\begin{array}{l}\text { - Midground views of opposing ridges; rustio } \\
\text { valleys, little to no long views }\end{array}$ \\
\hline Pair & $\begin{array}{l}\text { - Midground to foreground views of opposing } \\
\text { ridges, close views of roads, traffic; } \\
\text { development may bo present }\end{array}$ \\
\hline
\end{tabular}

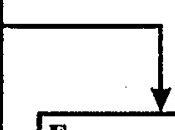

Foreground Condition (pick one) Presentation of View
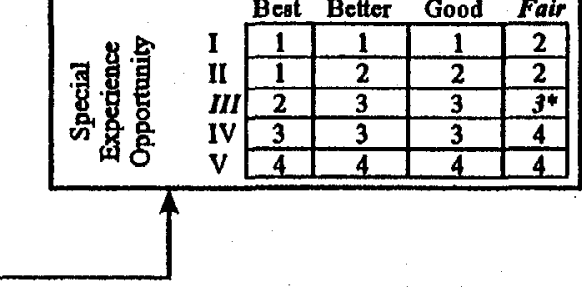

Opportunity of View (pick one)

A. Horizontal view angle $>180^{\circ}$; vertical view angle $>20^{\circ}$; pull over present

B. Horizontal view angle $90-180^{\circ}$; vertical view angle $15-20^{\circ}$; pull over present

C. Horizontal view angle $45-90^{\circ}$; vertical angle $10-15^{\circ}$; pull over available; larger viewing angles with no pull over available

D.* Horizontal view angle $30-45^{\circ}$; no pull over available

E. Horizontal viow anglo $<30^{\circ}$; no pull over

\section{Aesthetic Experience (pick one)}

Quality of View

Very

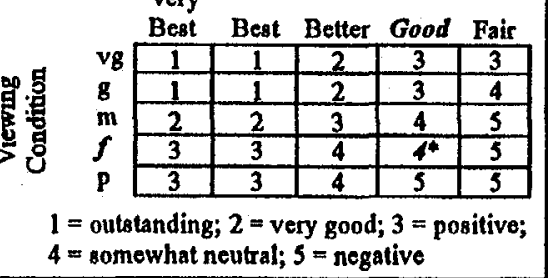

$4=$ somewhat neutral; $5=$ negative

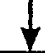

Vlewing Condition (pick one)

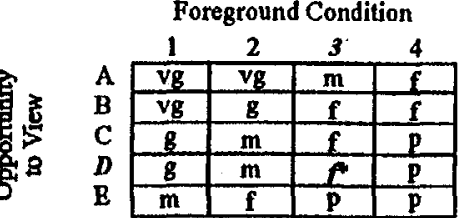

$v g=$ very good; $g=$ good; $m=$ moderate; $p=$ poor 


\section{Aesthetic Experience Worksheet Foothills Parkway Extension 8-B *** Views From the Parkway Extension ***}

\section{View 'I', Texas Creek Valley Overview S, 12-670}

Special Experience Opportunity (pick one)

I. Fall color, spring blooms; water sounds; cool in summer, very special lighting effects

II. Morning or evening back/side lighting; mis ts; winter ice/frosts; deep woods; cool wet smell

III. Wildlife viewing (birds, deer, ete.); special geology; cool woods smell; big trees; unusual vegetation; noticeable ccological processes

$I V . *$ Mostly normal mountain vegetation; little unusual lighting effects or sensory experiences expected

V. Blinding sun; heavy fog; bad smells; bad traffic situation; noisy; strong winds; dead and dying vegetation; hot in summer; trash in view

Choices are marked with an anterisk.

\begin{tabular}{|c|c|}
\hline Qualit & of View (pick one) \\
\hline & $\begin{array}{l}\text { - Water scenes with long views of sories of } \\
\text { receding mountain ridges and valleys }\end{array}$ \\
\hline Best* & $\begin{array}{l}\text { - Long views of receding mountain ridges or } \\
\text { water scenes but not both; close views of } \\
\text { wooded mountain streams. }\end{array}$ \\
\hline Better & $\begin{array}{l}\text { - Long but partially blocked viows of mountain } \\
\text { ridges or water scenes; more midground ridges } \\
\text { than long view }\end{array}$ \\
\hline & $\begin{array}{l}\text { - Midground viows of opposing ridges; nustic } \\
\text { valleys, little to no long views }\end{array}$ \\
\hline Fair & $\begin{array}{l}\text { - Midground to foreground views of opposing } \\
\text { ridges, close views of roads, traffic; } \\
\text { dovelopment may be present }\end{array}$ \\
\hline
\end{tabular}

Aesthetic Experience (pick one) Quality of View

Very

Foreground Condition (pick one) Prenentation of View
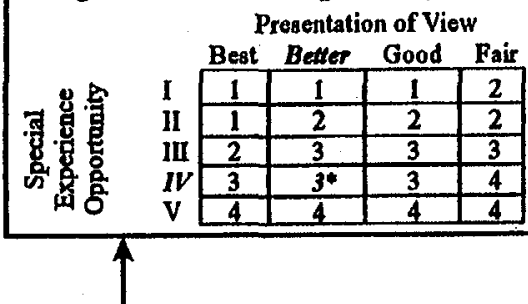

照害 Best Best Better Good Fair \begin{tabular}{|c|c|c|c|c|}
\hline 1 & 1 & 2 & 3 & 3 \\
\hline 1 & 1 & 2 & 3 & 4 \\
\hline
\end{tabular} \begin{tabular}{|l|l|l|l|l|}
\hline 2 & 2 & 3 & 4 & 5 \\
\hline & 3 & 4 & 4 & 5 \\
\hline
\end{tabular}

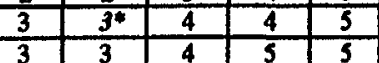
= outstanding; 2 = very good; $3=$ positive $4=$ somewhat neutral; $5=$ negative
Opportunity of View (pick one)

A. Horizontal view angle $>180^{\circ}$; vertical view angle $>20^{\circ}$; pull

B. Horizontal view angle $90-180^{\circ}$; vertical view angle $15-20^{\circ}$; pull over present

C. Horizontal view angle $45-90^{\circ}$; vertical angle $10-15^{\circ}$; pull over available; larger viewing angles with no pull over available

D.* Horizontal view angle $30-45^{\circ}$; no pull over available

E. Horizontal view angle $<30^{\circ}$; no pull over
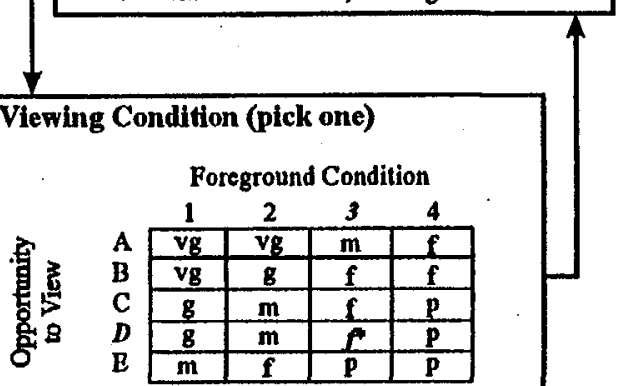

$v g=$ very good; $g$ = good; $m$ = moderate; $p=$ poor 


\section{Aesthetic Experience Worksheet \\ Foothills Parkway Extension 8-B} *** Views From the Parkway Extension ***

\section{View '11', Evergreen View N, 12-750}

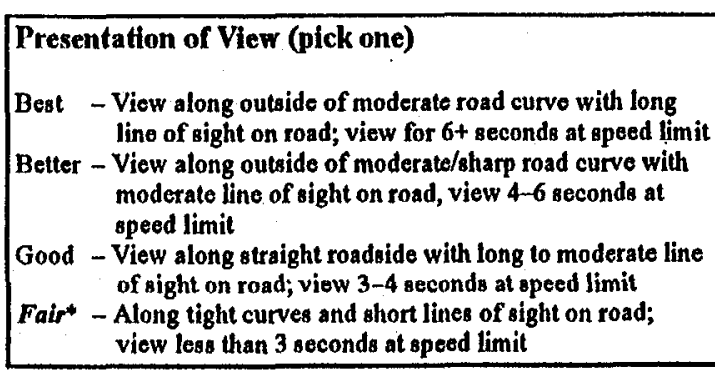

Special Experience Opportunity (pick one)

I. Fall color, spring blooms; water sounds; cool in summer, very special lighting effects

II. Morning or evening back/side lighting; mists; winter ice/frosta; deep woods; cool wet smells

III. * Wildtife viewing (birds, deer, etc.); special geology; cool woods smell; big trees; unusual vegetation; noticeable ecological processes

IV. Mostly normal mountain vegetation; little unuaual lighting effects or sensory experiences expected

Blinding aun; heavy fog; bad amells; bad traffic aituation; noiny; summer; trash in view

Choices are marked with an asterisk.

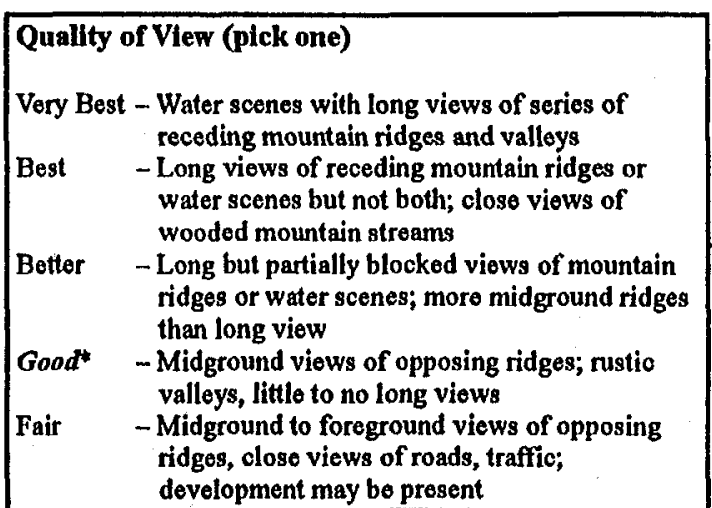
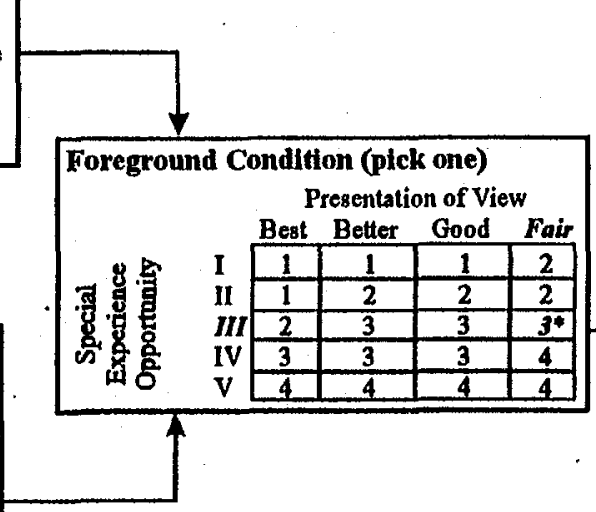

Opportunity of View (pick one)

A. Horizontal view angle $>180^{\circ}$; vertical view angle $>20^{\circ}$; pull over present

B. Horizontal view angle $90-180^{\circ}$; vertical view anglo $15-20^{\circ}$; pull over present

C. Horizontal viow angle $45-90^{\circ}$; vertical angle $10-15^{\circ}$; pull over available; larger viewing angles with no pull over avale

D. Horizontal view angle $30-45^{\circ}$; no pull over availablo E.* Horizontal view anglo $<30^{\circ}$; no pull over
Aesthetic Experience (pick one)

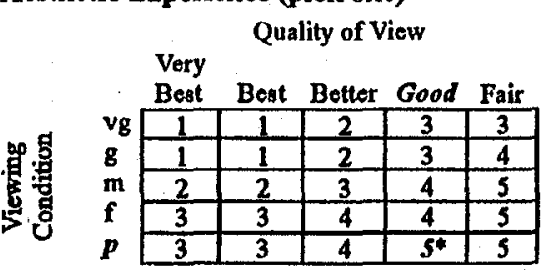

$1=$ outatanding; $2=$ very good; $3=$ positive; $4=$ somewhat neutral; $5=$ negativo
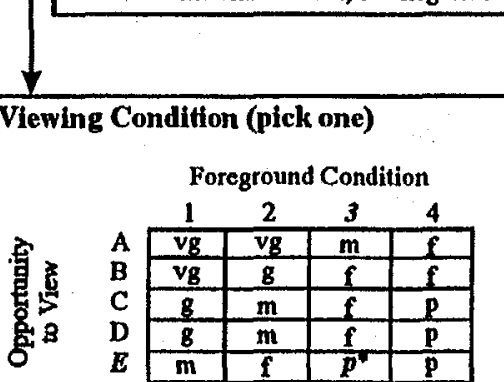

$v g=$ very good; $g=$ good; $m=$ moderate; $p=$ poor 


\section{Aesthetic Experience Worksheet}

Foothills Parkway Extension 8-B

*** Views From the Parkway Extension ***

\section{View '12', Valley Cove Mountain View, 13-250 to 13-450}

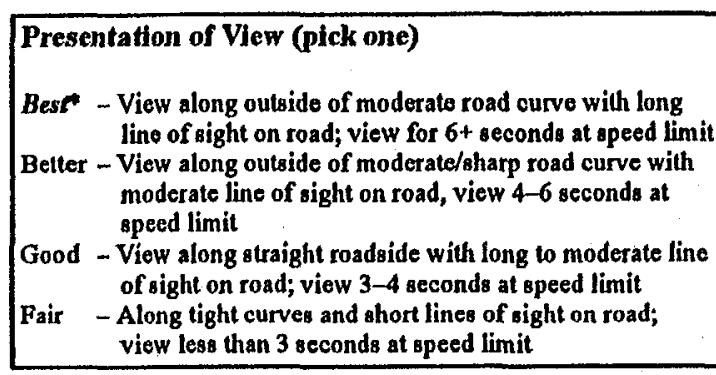

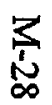

Special Experience Opportunity (pick one)

Fall color, spring blooms; water sounds; cool in aummer very special lighting effects

II. Moming or evening back/oide lighting; mists; winter ice/frosts; deep woods; cool wet smells

III. Wildlife viewing (birds, deer, etc.); special geology; cool wooda smell; big trees; unusual vegetation; noticeable ecological processes

IV.* Mostly normal mounta in vegetation; littlo unuaual lighting effects or sensory experiences expected

V. Blinding sun; heavy fog; bad smells; bad traffic situation; noisy; strong winds; dead and dying vegetation; hot in summer; trash in view

Choices are marked with an asterisk.

\begin{tabular}{|c|c|}
\hline Qualit & of Vlew (plck one) \\
\hline & - Water scenes with long views of series of \\
\hline Best* & $\begin{array}{l}\text { Long views of receding mountain ridges or } \\
\text { water scenes but not both; close views of } \\
\text { wooded mountain streams }\end{array}$ \\
\hline Better & $\begin{array}{l}\text { - Long but partially blocked viows of mountain } \\
\text { ridges or water scenes; moro midground ridges } \\
\text { than long view }\end{array}$ \\
\hline & $\begin{array}{l}\text { - Midground views of opposing ridges; rustic } \\
\text { valleys, little to no long viows }\end{array}$ \\
\hline Fair & $\begin{array}{l}\text { - Midground to foreground views of opposing } \\
\text { ridges, close views of roads, traffic; } \\
\text { dovelopment may be present }\end{array}$ \\
\hline
\end{tabular}

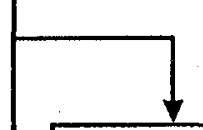

Foreground Condition (pick one)

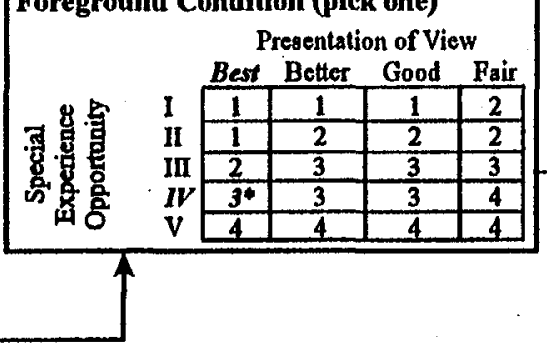

Opportunity of View (pick one)

A. Horizontal viow angle $>180^{\circ}$; vertical view angle $>20^{\circ}$; pull over present

$B . *$ Horizontal view angle $90-180^{\circ}$; vertical view angle $15-20^{\circ}$;

C. Horizontal view angle $45-90^{\circ}$; vertical angle 10-15०; pull Horizontal view angle $45-90^{\circ}$; vertical anglo $10-15^{\circ}$; pull
over available; larger viewing anglea with no pull over available

E. Horizontal view angle $<30^{\circ}$; no pull over
D. Horizontal view angle $30-45^{\circ}$; no pull over available 


\section{Aesthetic Experience Worksheet Foothills Parkway Extension 8-B *** Views From the Parkway Extension ***}

View '13', Little no-name Gap, 13-700
Choices are marked with an asterisk

\section{Special Expertence Opportunity (pick one)}

Fall color, spring blooms; water sounds; cool in summer, very special lighting effects

Morning or evening back/side lighting; mists; winter

ice/frosts; deep woods; cool wet smells

Wildlife viewing (birds, deer, etc.); special geology; cool woods smell; big trees; unusual vegetation; noticeable ecological processes

IV. Mostly normal mountain vegetation; little unusual lighting effects or sensory experiences expecled

Blinding sun; heavy fog; bad smells; bad traffic situation; noisy; strong winds; dead and dying vegetation; hot in summer; trash in view

\begin{tabular}{|c|c|}
\hline \multicolumn{2}{|c|}{ Quality of View (pick one) } \\
\hline Very Be & $\begin{array}{l}\text { - Water scenes with long views of series of } \\
\text { receding mountain ridges and valleys }\end{array}$ \\
\hline Best & $\begin{array}{l}\text { - Long views of receding mountain ridges or } \\
\text { water scenes but not both; close views of } \\
\text { wooded mountain streams }\end{array}$ \\
\hline Better" & $\begin{array}{l}\text { - Long but partially blocked views of mountain } \\
\text { ridges or water scenes; more midground ridges } \\
\text { than long view }\end{array}$ \\
\hline & $\begin{array}{l}\text { - Midground views of opposing ridges; nustio } \\
\text { valleys, little to no long viows }\end{array}$ \\
\hline Fair & $\begin{array}{l}\text { - Midground to foreground views of opposing } \\
\text { ridges, olose views of roads, traffic; } \\
\text { development may be present }\end{array}$ \\
\hline
\end{tabular}

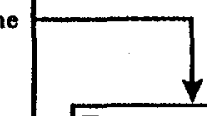

Foreground Condition (pick one)

Presentation of View
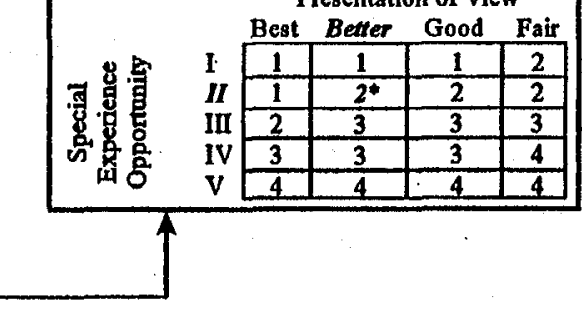

Opportunity of View (pick one)

A. Horizontal view angle $>180^{\circ}$; vertical view angle $>20^{\circ}$; pull

B. Horizontal view angle $90-180^{\circ}$; vertical view angle $15-20^{\circ}$; pull over present

C. Horizontal view angle $45-90^{\circ}$; vertical anglo $10-15^{\circ}$; pull over available; larger viowing angles with no pull over available

D. Horizontal view anglo $30-45^{\circ}$; no pull over available

B. Horizontal view angle $<30^{\circ}$; no pull over

\section{Aesthetic Expertence (pick one)}

Quality of View Very

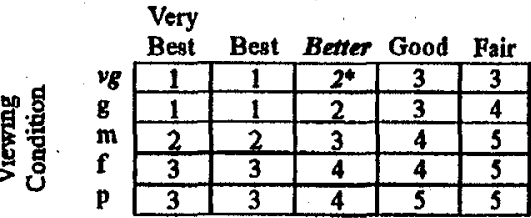

1 = outstanding; 2 = very good; 3 = positive; $4=$ somewhat neutral; $5=$ negative

$\downarrow$

Viewing Condition (pick one)

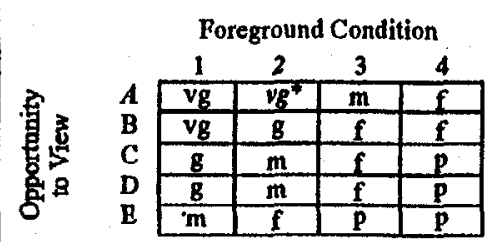

$\mathrm{Vg}=$ very good; $g=$ good; $m=$ moderate; $p=$ poor

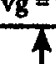

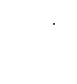

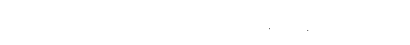




\section{Aesthetic Experience Worksheet Foothills Parkway Extension 8-B *** Views From the Parkway Extension ***}

\section{View 'K, Shults Grove Church, 16-350 to 16-570}

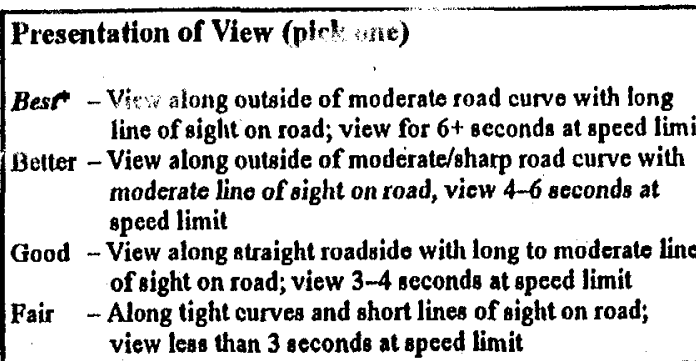

Besf - Viecizalong outside of moderate road curve with long line of siglit on road; view for $6+$ seconds at speed limit Better - View along outside of moderate/sharp road curve with moderate line of sight on road, view 4-6 seconds at speed limit

Good - View along straight roadside with long to moderate line of sight on road; view 3-4 seconds at apeed limit

Fair - Along tight curves and short lines of sight on road view less than 3 seconds at speed limit

Special Experience Opportunity (pick one)

I. Fall color, spring blooms; water sounds; cool in summer very special lighting effects

1I. Moming or evening back/side lighting; mists; winter ice/frosts; deep woods; cool wet smells

III. * Wildlife viewing (birds, deer, etc.); special geology; cool woods smell; big trees; unusual vegetation; noticeable ccological processes

IV. Mostly normal mountain vegetation; little unusual lighting offects or sensory oxperiences expected

Blinding aun; heavy fog; bad smello; bad traffic situation; noisy; strong winds; dead and dying vegetation; hot in summer; trash in view

Choices are marked with an asterisk.

\begin{tabular}{|c|c|}
\hline \multicolumn{2}{|c|}{ Quality of $V$ t (pick one) } \\
\hline \multicolumn{2}{|c|}{$\begin{array}{l}\text { mest - Water scenes with long views of series of } \\
\text { receding mountain ridges and valleys }\end{array}$} \\
\hline Best & $\begin{array}{l}\text { - Long viows of receding mountain ridges or } \\
\text { water scenes but not both; close views of } \\
\text { wooded mountain streams }\end{array}$ \\
\hline Better & $\begin{array}{l}\text { - Long but partially blocked viows of mountain } \\
\text { ridges or water scenes; more midground ridges } \\
\text { than long viow }\end{array}$ \\
\hline & $\begin{array}{l}\text { - Midground views of opposing ridges; rustio } \\
\text { valleys, little to no long views }\end{array}$ \\
\hline & $\begin{array}{l}\text { - Midground to foreground views of opposing } \\
\text { ridges, close views of roads, traffic; } \\
\text { development may be present }\end{array}$ \\
\hline
\end{tabular}

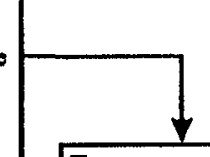

Foreground Condition (pick one)
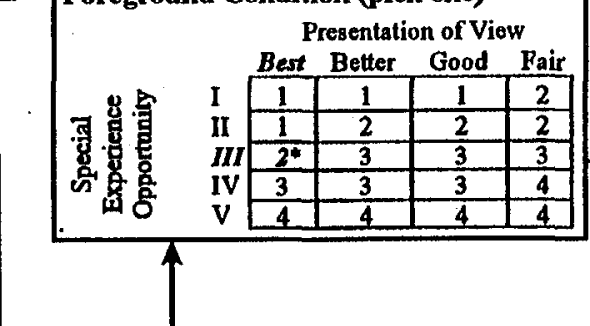

\section{Opportunity of Vlew (pick one)}

A. Horizontal view angle $>180^{\circ}$; vertical view angle $>20^{\circ}$; pull over present

B. Horizontal view angle $90-180^{\circ}$; vertical view angle $15-20^{\circ}$; pull over present

C.* Horizontal view angle $45-90^{\circ}$; vertical angle $10-15^{\circ}$; pull over available; larger viewing angles with no pull over available

D. Horizontal view angle $30-45^{\circ}$; no pull over available

E. Horizontal view angle $<30^{\circ}$; no pull over
Aesthetic Experience (pick one)

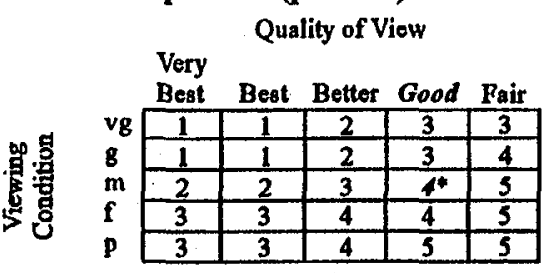

$=$ outstanding; $2=$ very good; $3=$ positive; $4=$ somewhat neutral; $5=$ negative

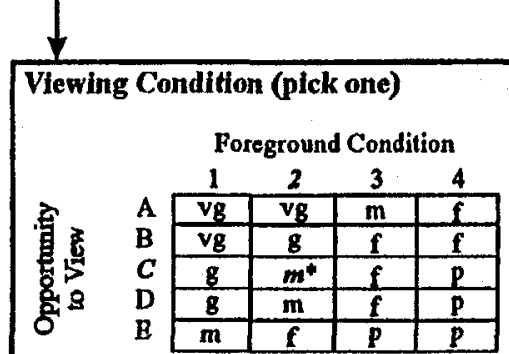

$v g=$ very good; $g=$ good; $m=$ moderate; $p=$ poor 


\section{Aesthetic Experience Worksheet \\ Foothills Parkway Extension 8-B \\ *** Views From the Parkway Extension ***}

\section{View 'L', Rocky Grove View S, 16-850 to 17-000}

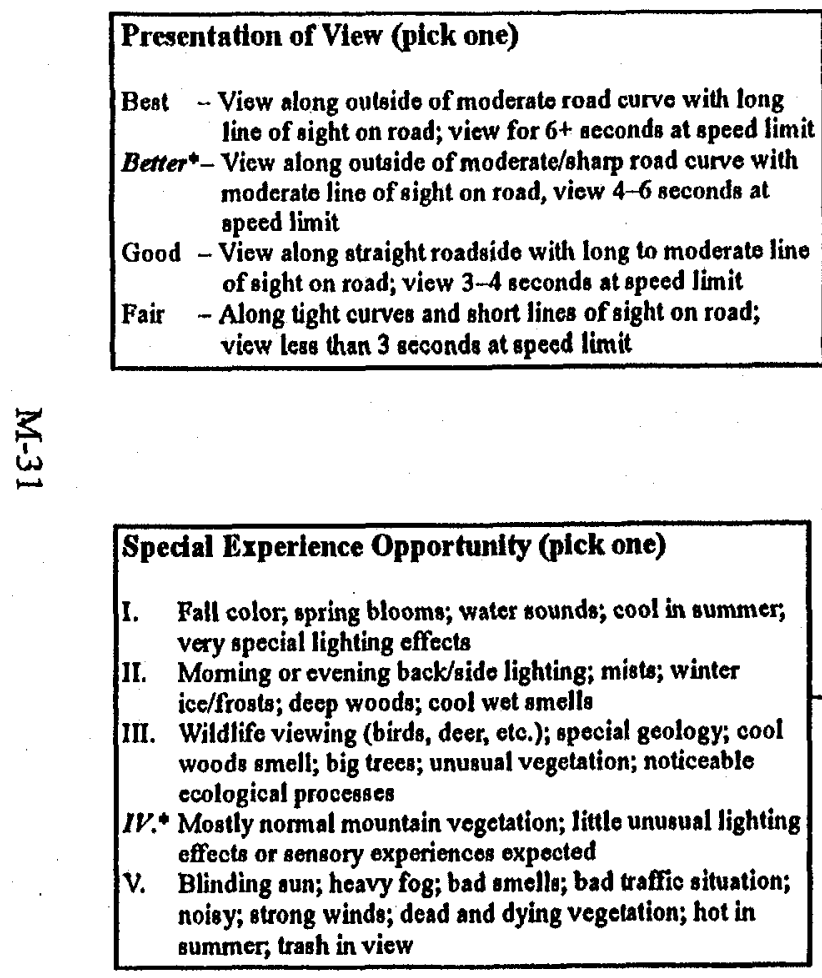

Choices are marked with an asterisk.

B. Horizontal view aver avalable

\begin{tabular}{|c|c|}
\hline \\
\hline \multicolumn{2}{|r|}{$\begin{array}{l}\text { - Water scenes with long views of series of } \\
\text { receding mountain ridges and valleys }\end{array}$} \\
\hline \multicolumn{2}{|r|}{$\begin{array}{l}\text { - Long viows of receding mountain ridges or } \\
\text { water scenes but not both; close views of } \\
\text { wooded mountain streams }\end{array}$} \\
\hline \multirow{3}{*}{$\begin{array}{l}\text { Better } \\
\text { Good } \\
\text { Fair }\end{array}$} & $\begin{array}{l}\text { - Long but partially blocked views of mountain } \\
\text { ridges or water scenes; more midground ridges }\end{array}$ \\
\hline & $\begin{array}{l}\text { - Midground views of opposing ridges; rustio } \\
\text { valleys, little to no long views }\end{array}$ \\
\hline & $\begin{array}{l}\text { - Midground to foreground views of opposing } \\
\text { ridges, close views of roads, traffic; } \\
\text { development may be present }\end{array}$ \\
\hline
\end{tabular}
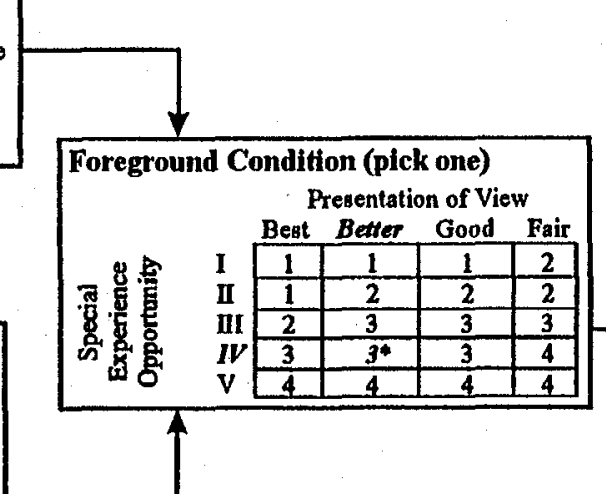
itation of View
Aesthetic Experience (pick one)

Quality of View

Very

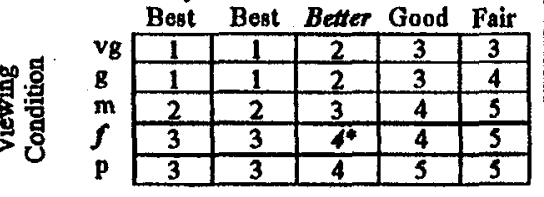

1 = outstanding; 2 = very good; 3 = positive; $4=$ somewhat neutral; $5=$ negative
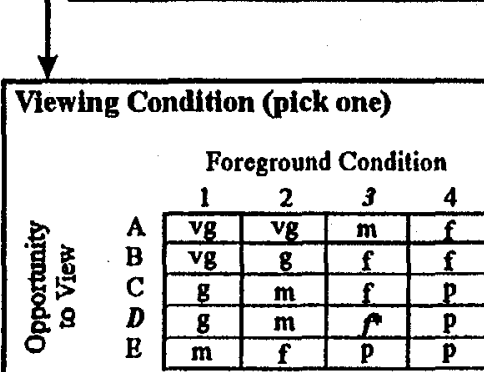

$v g=$ very good; $g=$ good; $m=$ moderato; $p=$ poor
D. Horizontal view angle $30-45^{\circ}$. no pull over available

B. Horizontal view angle $<30^{\circ}$; no pull over 


\section{Aesthetic Experience Worksheet Foothills Parkway Extension 8-B *** Views From the Parkway Extension ***}

\section{View 'M', Cole Creek S, 17-860}

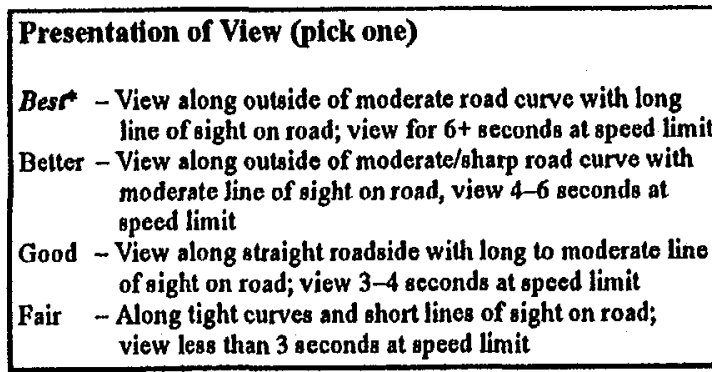

Special Experience Opportunity (pick one)

Fall color, spring blooms; water sounds; cool in summer very special lighting effects

II.* Morning or evening back/side lighting; mists; winter ice/frosts; deep woods; cool wet smells

III. Wildlife viewing (birds, deer, etc.); special geology; cool woods smell; big trees; unusual vegetation; noticeable ecological processes

IV. Mostly normal mountain vegetation; little unuaual lighting effects or sensory experiences expected

Blinding aun; heavy fog bad amello; bad traffic situation; noisy; strong winds; dead and dying vegetation; hot in summer; trash in view

\begin{tabular}{|c|c|}
\hline Quallty & of View (pick one) \\
\hline & $\begin{array}{l}\text { - Water scenes with long viows of series of } \\
\text { receding mountain ridges and valleys }\end{array}$ \\
\hline Best & $\begin{array}{l}\text { - Long views of receding mountain ridges or } \\
\text { water scenes but not both; close views of } \\
\text { wooded mountain streams }\end{array}$ \\
\hline Better* & $\begin{array}{l}\text { - Long but partially blocked viows of mountain } \\
\text { ridges or water scenos; more midground ridges } \\
\text { than long view }\end{array}$ \\
\hline & $\begin{array}{l}\text { - Midground viows of opposing ridges; rustio } \\
\text { valloys, littlo to no long views }\end{array}$ \\
\hline & $\begin{array}{l}\text { - Midground to foreground views of opposing } \\
\text { ridges, close views of roads, traffic; } \\
\text { development may be present }\end{array}$ \\
\hline
\end{tabular}
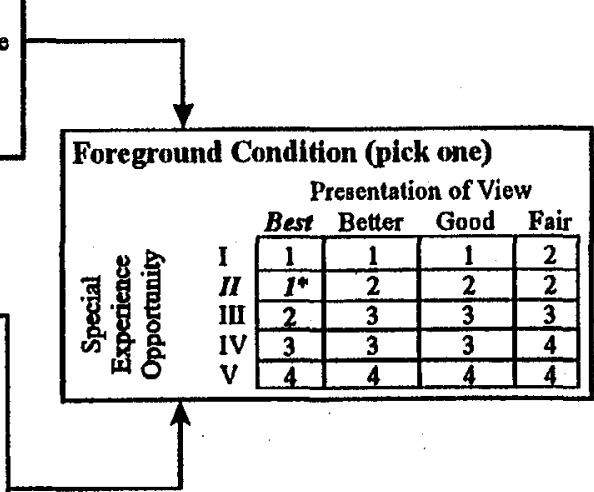

\section{Opportunity of View (pick one)}

A. Horizontal view angle $>180^{\circ}$; vertical view angle $>20^{\circ}$; pull over present

B. Horizontal view angle $90-180^{\circ}$; vertical view angle $15-20^{\circ}$ pull over present

C. Horizontal view anglo $45-90^{\circ}$; vertical anglo $10-15^{\circ}$; pull over available; larger viewing angles with no pull over available

D. Horizontal view angle $30-45^{\circ}$; no pull over availablo E.* Horizontal view angle $<30^{\circ}$; no pull over

\section{Aesthetic Experience (pick one)} Quality of View

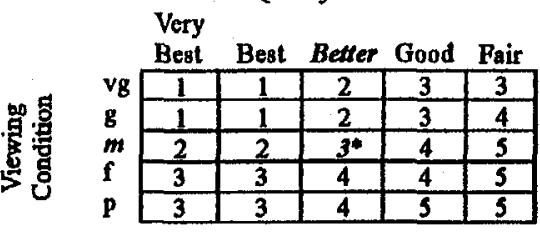

= outstanding; $2=$ very good; $3=$ positive $4=$ somewhat neutral; $5=$ negative

$\downarrow$

Vlewing Condition (pick one)

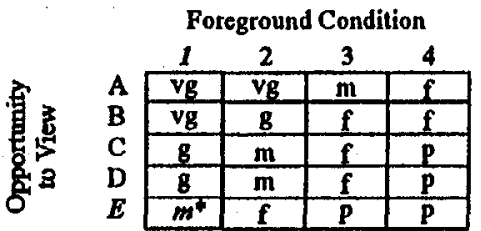

$v g=$ very good; $B=$ good; $m=$ moderate; $p=$ poor 


\section{Aesthetic Experience Worksheet} Foothills Parkway Extension 8-B *** Views From the Parkway Extension $* * *$

\section{View '14', Buckeye Creek S, 18-300}

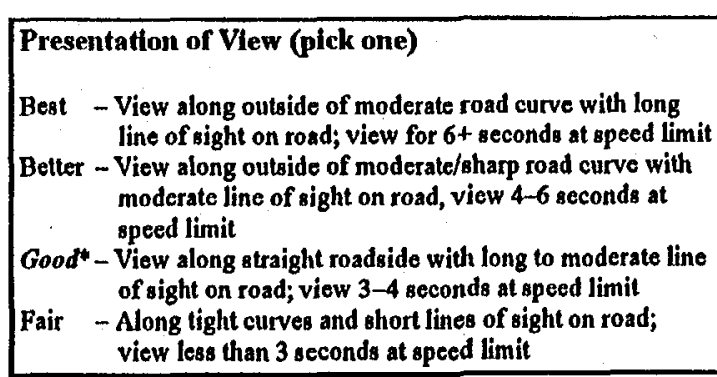

忑.

Special Experience Opportunity (pick one)

I. Fall color, spring blooms; water sounds; cool in summer; very special lighting effects

Moming or ovening back/side lighting; mists; winter ice/frosta; deep woods; cool wet smells

III. Wildlifo viewing (birds, deer, etc.); special geology; cool woods amell; big trees; unusual vegetation; noticeablo ecological processes

IV.* Mostly normal mountain vegetation; little unusual lighting effects or sensory experiences expected

v. Blinding sun; heavy fog; bad omells; bad traffic situation; noisy; strong winds; dead and dying vegetation; hot in oummer; trash in view

Choices are marked with an asterisk.

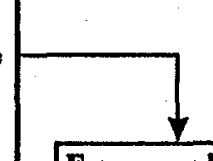

\begin{tabular}{|c|c|}
\hline \multicolumn{2}{|c|}{ Quality of View (pick one) } \\
\hline \multicolumn{2}{|c|}{$\begin{array}{l}\text { Very Best - Water scenes with long views of series of } \\
\text { receding mountain ridges and valloys }\end{array}$} \\
\hline Best* & $\begin{array}{l}\text { - Long views of receding mountain ridges or } \\
\text { water scenes but not both; close views of } \\
\text { wooded mountain streams }\end{array}$ \\
\hline Better & $\begin{array}{l}\text { - Long but partially blocked views of mountain } \\
\text { ridges or water scenes; moro midground ridges } \\
\text { than long view }\end{array}$ \\
\hline & $\begin{array}{l}\text { - Midground views of opposing ridges; rustic } \\
\text { valleys, little to no long viows }\end{array}$ \\
\hline Fair & $\begin{array}{l}\text { - Midground to foreground views of opposing } \\
\text { ridges, close views of roads, traffic; } \\
\text { development may be present }\end{array}$ \\
\hline
\end{tabular}

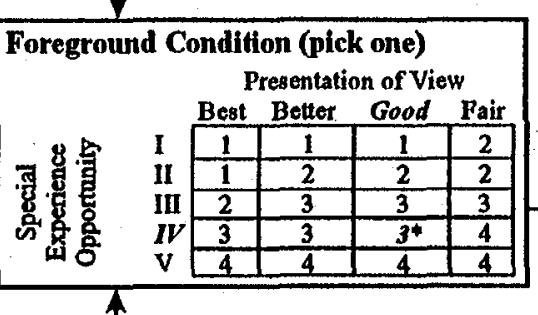
Presentation of $V$ 


\section{Aesthetic Experience Worksheet Foothills Parkway Extension 8-B *** Views From the Parkway Extension}

\section{View 'N', Indian Creek E, 18-850}

Choices are marked with an asterisk.

\section{Presentation of View (pick one)}

Best - View along outside of moderate road curve with long line of aight on road; view for $6+$ seconds at speed lim Better - Viow along outside of moderate/sharp road curve with moderate line of sight on road, view 4-6 seconds at speed limit

Good - View along straight roadside with long to moderate line of sight on road; view 3-4 aeconds at speed limit - Along tight curves and short lines of gight on road view less than 3 seconds at speed limit

\section{Speclal Experience Opporfunity (pick one)}

Fall color, opring blooms; water sounds; cool in summer very special lighting effects

Moming or evening back/side lighting; mists; winter iceifrosts; deep woods; cool wel smells

Wildife viewing (birds, deer, etc.); special geology; cool woods smell; big trees; unusual vegetation; noticeable ecological processes

V. Mostly normal mountain vegetation; little unuasual lighting effects or sensory experiences expected

V. Blinding sun; heavy fog; bad smells; bad traffic situation; noisy; atrong winds; dead and dying vegetation; hot in summer; trash in view

\begin{tabular}{|c|c|}
\hline \\
\hline \multicolumn{2}{|c|}{$\begin{array}{l}\text { Quality of View (pick one) } \\
\text { Very Best - Water scenes with long views of series of } \\
\text { receding mountain ridges and valloys }\end{array}$} \\
\hline \multicolumn{2}{|c|}{ Best - Long views of receding mountain ridges or } \\
\hline $\begin{array}{l}\text { Better } \\
\text { Good* } \\
\text { Pair }\end{array}$ & $\begin{array}{l}\text { - Long but partially blocked views of mountain } \\
\text { ridges or water scenes; more midground ridges } \\
\text { than long viow }\end{array}$ \\
\hline \multirow{2}{*}{$\begin{array}{l}\text { Good* } \\
\text { Pair }\end{array}$} & $\begin{array}{l}\text { - Midground views of opposing ridges; rustio } \\
\text { valleys, little to no long views }\end{array}$ \\
\hline & $\begin{array}{l}\text { - Midground to foreground views of opposing } \\
\text { ridges, close views of roads, traffic; } \\
\text { dovelopment may be present }\end{array}$ \\
\hline
\end{tabular}

$\downarrow$
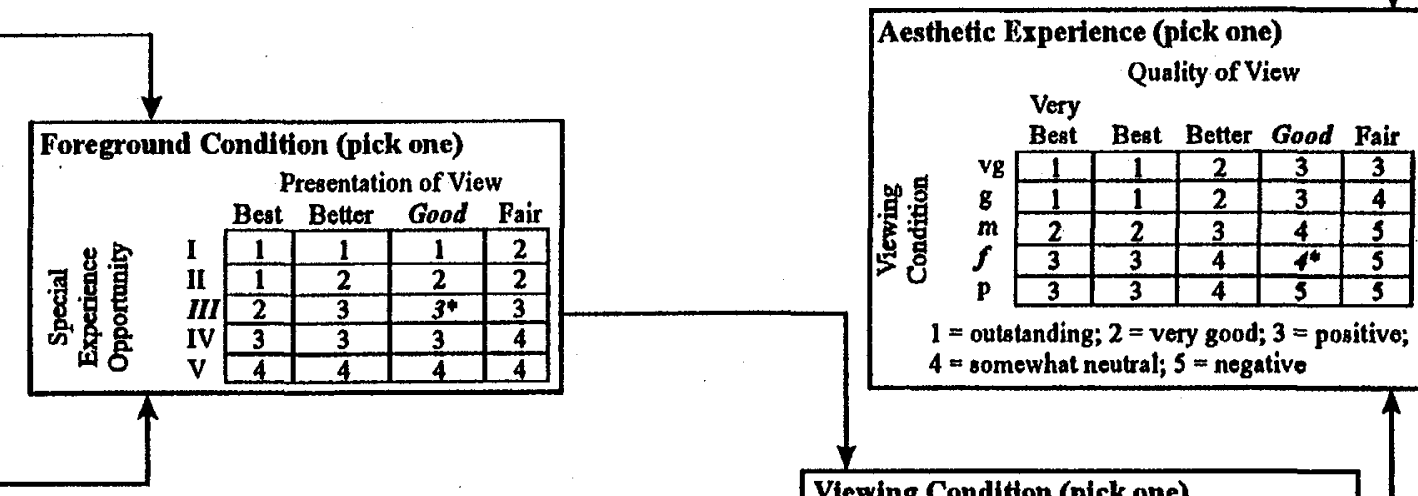

$1=$ outstanding; 2 = very good; 3 = positive; $4=$ somewhat neutral; $5=$ negative

Opportunity of View (pick one)

A. Horizontal view angle $>180^{\circ}$; vertical view angle $>20^{\circ}$; pull over present

B. Horizontal view angle $90-180^{\circ}$; vertical view angle $15-20^{\circ}$; pull over present

C. Horizontal view angle $45-90^{\circ}$; vertical angle $10-15^{\circ}$; pull over available; larger viewing angles with no pull over available

D.* Horizontal view angle $30-45^{\circ}$; no pull over available

B. Horizontal view angle $<30^{\circ}$; no pull over

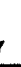

Viewing Condition (pick one)

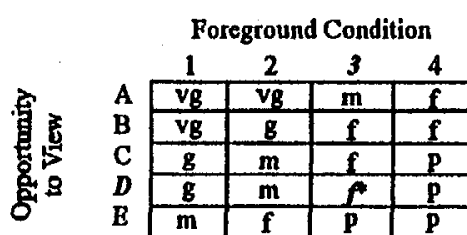

$\mathrm{vg}=$ very good; $g=$ good; $m$ = moderale; $p=$ poor

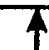




\section{Aesthetic Experience Worksheet Foothills Parkway Extension 8-B *** Views From the Parkway Extension ***}

\section{View '15', Sandy Hollow E, 19-650}

\begin{tabular}{|c|}
\hline Presentation of View (pick one) \\
Best - View along outside of moderate road curve with long \\
line of sight on road; view for $6+$ seconds at speed limit \\
Better - View along outside of moderate/sharp road curve with \\
moderate lino of sight on road, view $4-6$ seconds at \\
speed limit \\
Good* - View along straight roadside with long to moderate line \\
of aight on road; view $3-4$ seconds at speed limit \\
Fair - Along tight curves and short lines of sight on road; \\
view less than 3 seconds at speed limit
\end{tabular}

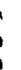

\begin{tabular}{|l}
\hline Special Experience Opportunity (pick one) \\
I. Fall color; spring blooma; water sounds; cool in summer, \\
very special lighting effects \\
II. Morming or evening back/side lighting; mists; winter \\
ice/frosts; deep woods; cool wet smella \\
III. Wildlife viewing (birds, deer, etc.); special geology; cool \\
woods smell; big trees; unusual vegetation; noticeable \\
ecological processes \\
IV. Mostly normal mountain vegetation; little unusual lighting \\
effects or sensory experiences expected \\
V. Blinding sun; heavy fog; bad smells; bad traffic situation; \\
noisy; strong winds; dead and dying vegetation; hot in \\
summer; trash in view
\end{tabular}

Choicen are marked with an asterisk.

\begin{tabular}{|c|c|}
\hline Qualit & of View (pick one) \\
\hline & $\begin{array}{l}\text { - Water scenes with long views of series of } \\
\text { receding mountain ridges and valloys }\end{array}$ \\
\hline Best & $\begin{array}{l}\text { - Long views of receding mountain ridges or } \\
\text { water scenes bul not both; close views of } \\
\text { wooded mountain streams }\end{array}$ \\
\hline Better & $\begin{array}{l}\text { - Long but partially blocked views of mountain } \\
\text { ridges or water scenes; more midground ridges } \\
\text { than long view }\end{array}$ \\
\hline & $\begin{array}{l}\text { - Midground views of opposing ridges; rustic } \\
\text { valleys, little to no long views }\end{array}$ \\
\hline Fair & $\begin{array}{l}\text { - Midground to foreground views of opposing } \\
\text { ridges, close views of roads, traffic; } \\
\text { development may be present }\end{array}$ \\
\hline
\end{tabular}

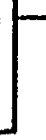
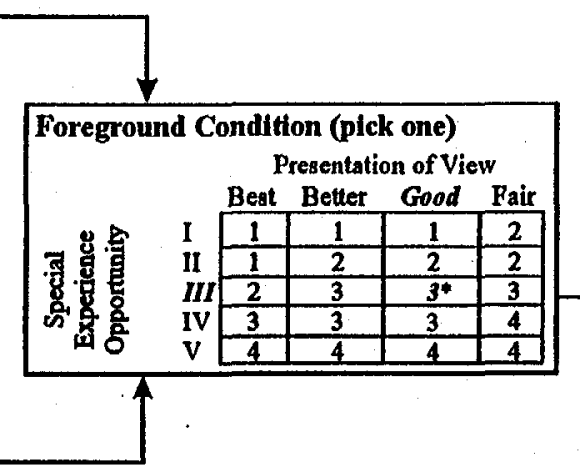

\section{Aesthetic Expertence (pick one) Quality of View} Very

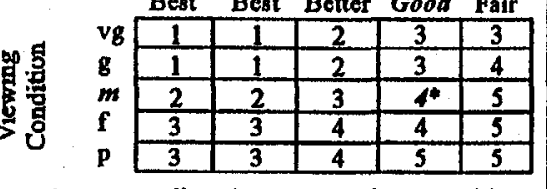

1 = outstanding; 2 = very good; 3 = positive; $4=$ somewhat neutral; $5=$ negative

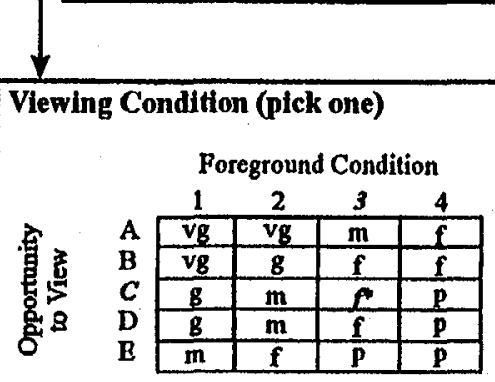

Opportunity of View (pick one)

A. Horizontal view angle $>180^{\circ}$; vertical view angle $>20^{\circ}$; pull over present

B. Horizontal view angle $90-180^{\circ}$; vertical view angle $15-20^{\circ}$; pull over present

C.* Horizontal view anglo $45-90^{\circ}$; vertical angle $10-15^{\circ}$; pull over availablo; larger viewing angles with no pull over available

D. Horizontal view angle $30-45^{\circ}$; no pull over available

B. Horizontal view angle $<30^{\circ}$; no pull over

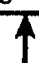

$v g=$ very good; $g=$ good; $m=$ moderate; $p=$ poor 


\section{Aesthetic Experience Worksheet Foothills Parkway Extension 8-B *** Views From the Parkway Extension View '16', Chavis Creek W, 19-800 to 20-000}

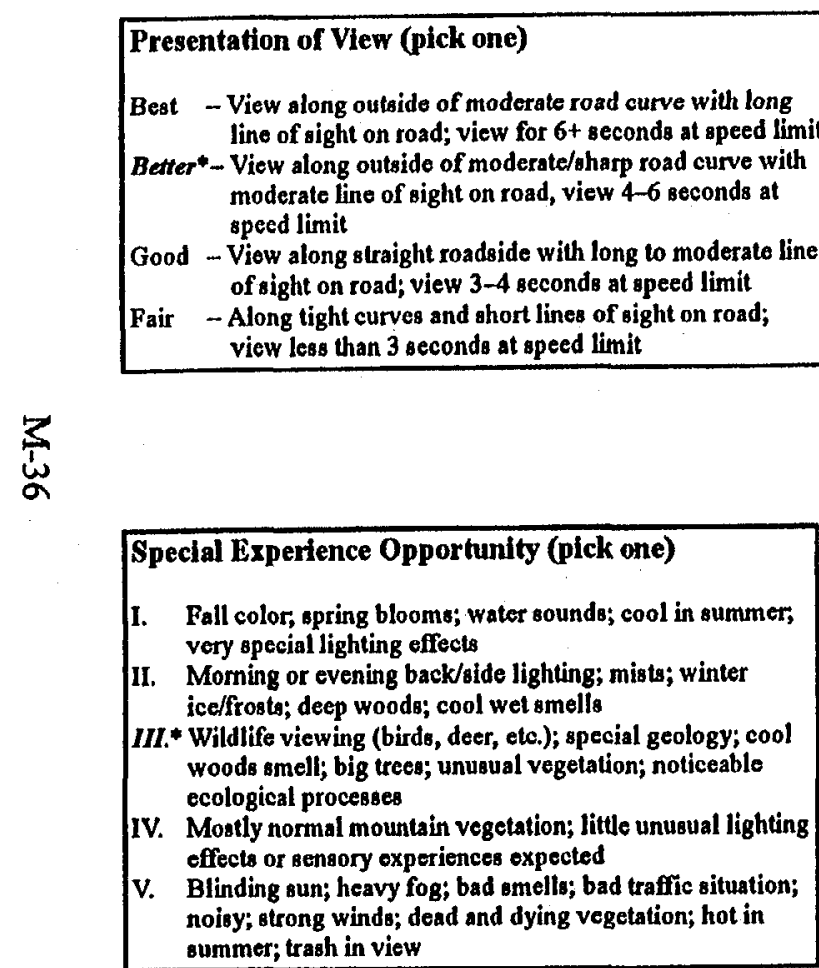

Choices are marked with an asterisk.

\begin{tabular}{|c|c|}
\hline \multicolumn{2}{|c|}{ Quality of View (pick one) } \\
\hline \multicolumn{2}{|c|}{$\begin{array}{c}\text { Very Best - Water scenes with long views of series of } \\
\text { receding mountain ridges and valleys }\end{array}$} \\
\hline & $\begin{array}{l}\text { - Long viows of receding mountain ridges or } \\
\text { water scenes but not both; closo views of } \\
\text { wooded mountain streams }\end{array}$ \\
\hline & $\begin{array}{l}\text { - Long but partially blocked views of mountain } \\
\text { ridges or water scenes; more midground ridges } \\
\text { than long view }\end{array}$ \\
\hline & $\begin{array}{l}\text { - Midground views of opposing ridges; rustic } \\
\text { valleys, fittle to no long views }\end{array}$ \\
\hline & $\begin{array}{l}\text { - Midground to foreground views of opposing } \\
\text { ridges, close views of roads, traffic; } \\
\text { development may be present }\end{array}$ \\
\hline
\end{tabular}

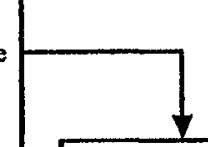

Foreground Condition (pick one)

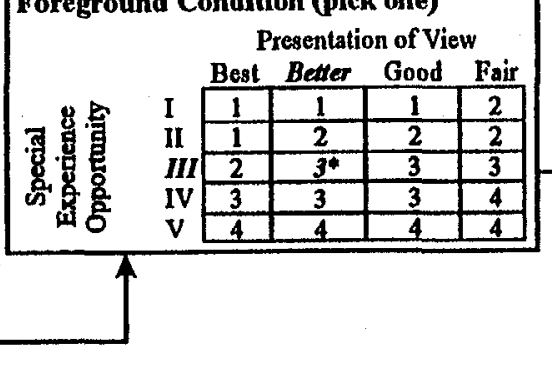

Opportunity of View (pick one)

A. Horizontal viow angl $>180^{\circ}$; vertical view angle $>20^{\circ}$; pull over present

B. Horizontal view angle $90-180^{\circ}$; vertical view angle $15-20^{\circ}$; pull over present

C.* Horizontal view anglo $45-90^{\circ}$; vertical angle $10-15^{\circ}$; pull over available; langer viewing angles with no pull over available

D. Horizontal view angle $30-45^{\circ}$; no pull over available

B. Horizontal view angle $<30^{\circ}$; no pull over

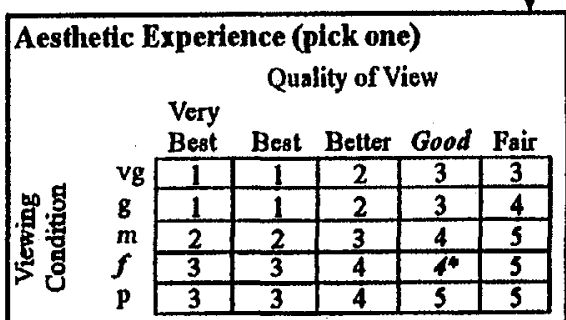

1 = outstanding; 2 = very good; 3 = positive; $4=$ somewhat neutral; 5 = negative

$\downarrow$

Viewing Condition (pick one)

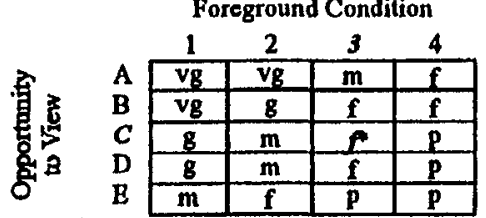

$v g=$ very good; $g$ = good; $m$ = moderate; $p$ = poor 


\section{Aesthetic Experience Worksheet \\ Foothills Parkway Extension 8-B *** Views From the Parkway Extension ***}

View '17', Big Ridge N, 20-500

\begin{tabular}{|c|c|}
\hline Qualit & of View (pick one) \\
\hline Very Be & $\begin{array}{l}\text { - Water scenes with long views of series of } \\
\text { receding mountain ridges and valleys }\end{array}$ \\
\hline Best & $\begin{array}{l}\text { - Long views of receding mountain ridges or } \\
\text { water scenes but not both; close views of } \\
\text { wooded mountain streams }\end{array}$ \\
\hline Better & $\begin{array}{l}\text { - Long but partially blocked views of mountain } \\
\text { ridges or water scenes; moro midground ridges } \\
\text { than long view }\end{array}$ \\
\hline & $\begin{array}{l}\text { - Midground viows of opposing ridges; nustio } \\
\text { valleys, little to no long viows }\end{array}$ \\
\hline & $\begin{array}{l}\text { - Midground to foreground views of opposing } \\
\text { ridges, close viows of roads, traffic; } \\
\text { development may be present }\end{array}$ \\
\hline
\end{tabular}

Presentation of View (pick one)

Best - View along outside of moderate road curvo with long line of sight on road; view for $6+$ seconds at apeed limit

Better - View along outside of moderate/sharp road curve with moderate line of sight on road, view 4-6 seconds at speed limit

Good - View along straight roadside with long to moderate lino of sight on road; view 3-4 seconds at speed limit

Fair* - Along tight curves and short lines of sight on road; view less than 3 seconds at speed limit

\begin{tabular}{|l|}
\hline Special Experience Opportunity (pick one) \\
I. \\
Iall color, spring blooms; water sounds; cool in summer; \\
very special lighting effects \\
II. Morning or evening back/side lighting; mists; winter \\
ice/frosts; deep woods; cool wel smella \\
III. Wildlife viewing (birds, deer, ete.); special geology; cool \\
woods smell; big trees; unusual vegetation; noticesble \\
ecological processes \\
IV.* Mostly normal mountain vegetation; littlo unusual lighting \\
effects or sensory experiences expected \\
V. $\begin{array}{l}\text { Blinding sun; heavy fog; bad smells; bad traffic situation; } \\
\text { noiay; strong winds; dead and dying vegetation; hot in } \\
\text { oummer; trash in view }\end{array}$
\end{tabular}

Choices are marked with an asterisk.

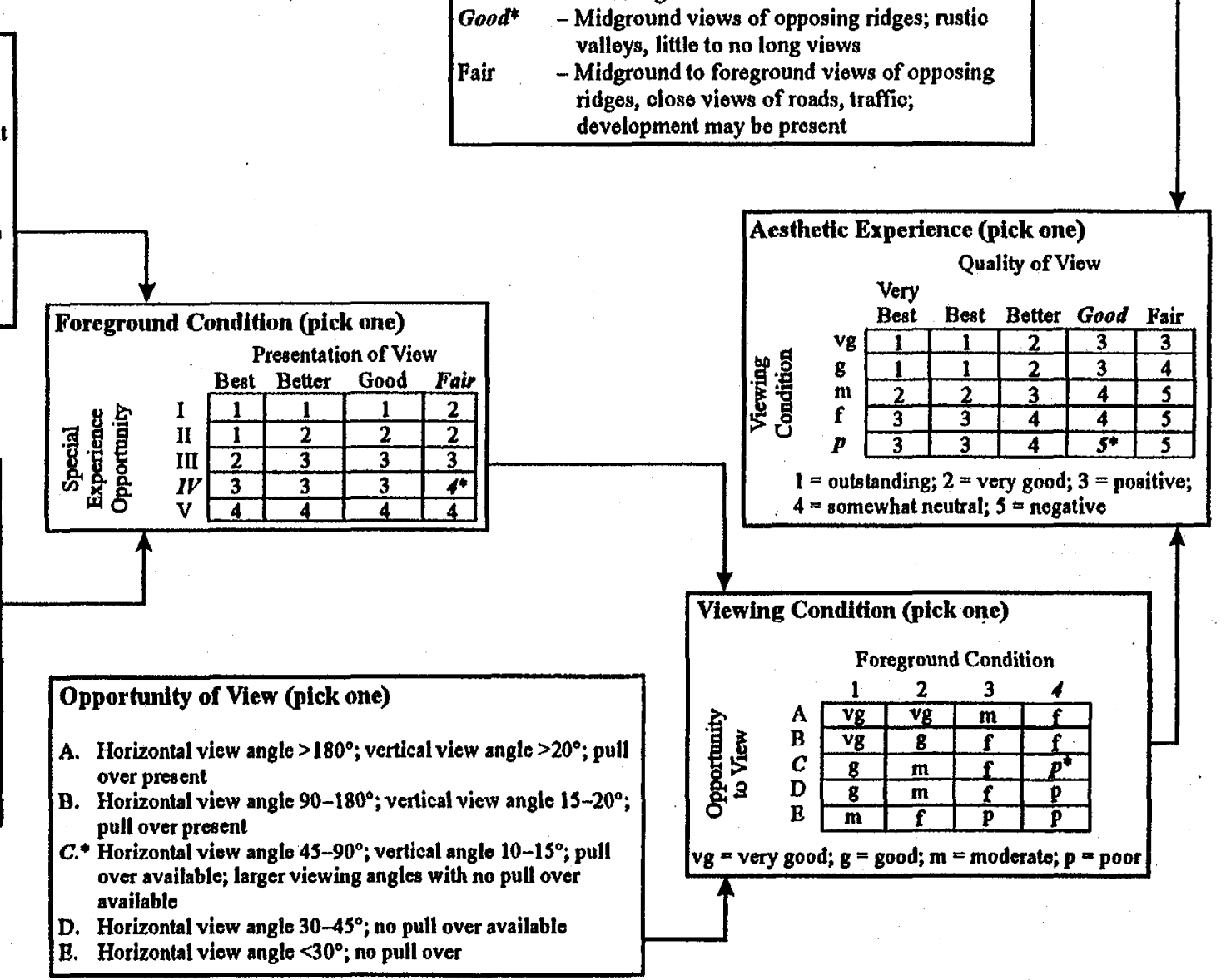

D. Horizontal view angle $30-45^{\circ}$; no pull over available

B. Horizontal view angle $<30^{\circ}$; no pull over 


\section{Aesthetic Experience Worksheet}

Foothills Parkway Extension 8-B

*** Views From the Parkway Extension $* * *$

\section{View 'O', Camp Creek-Sandy Hollow SE, 21-200}

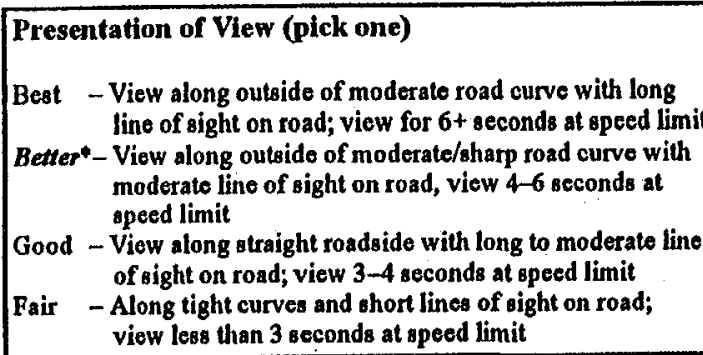

灾

Special Experience Opportunity (pick one)

Pall color; spring blooms; water sounds; cool in summer very special tighting effects

11.* Morning or evening back/side lighting; mists; winter ice/frosts; deep woods; cool wet amells

III. Wildlife viewing (birds, deer, etc.); special geology; cool woods smell; big trees; unusual vegetation; noticeable ecological processes

IV. Mostly normal mountain vegetation; littlo unusual lighting effects or sensory experiences expected

V. Blinding ann; heavy fog; bad smells; bad traffic situation; noisy; strong winda; dead and dying vegetation; hot in summer; tranh in view

Choices aro marked with an asterisk.

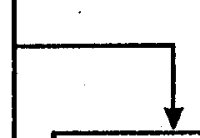
over present pull over present available

\begin{tabular}{|c|c|}
\hline Quality & of View (pick one) \\
\hline Very Bo & $\begin{array}{l}\text { - Water scenes with long views of series of } \\
\text { receding mountain ridges and valleys }\end{array}$ \\
\hline & $\begin{array}{l}\text { - Long views of receding mountain ridges or } \\
\text { water scenes but not both; close views of } \\
\text { wooded mountain streams }\end{array}$ \\
\hline Better* & $\begin{array}{l}\text { - Long but partially blocked viows of mountain } \\
\text { ridges or water scenes; more midground ridges } \\
\text { than long view }\end{array}$ \\
\hline & $\begin{array}{l}\text { - Midground views of opposing ridges; rustic } \\
\text { valleys, littlo to no long views }\end{array}$ \\
\hline Fair & $\begin{array}{l}\text { - Midground to foreground views of opposing } \\
\text { ridges, close views of roads, traffic; } \\
\text { development may bo present }\end{array}$ \\
\hline
\end{tabular}
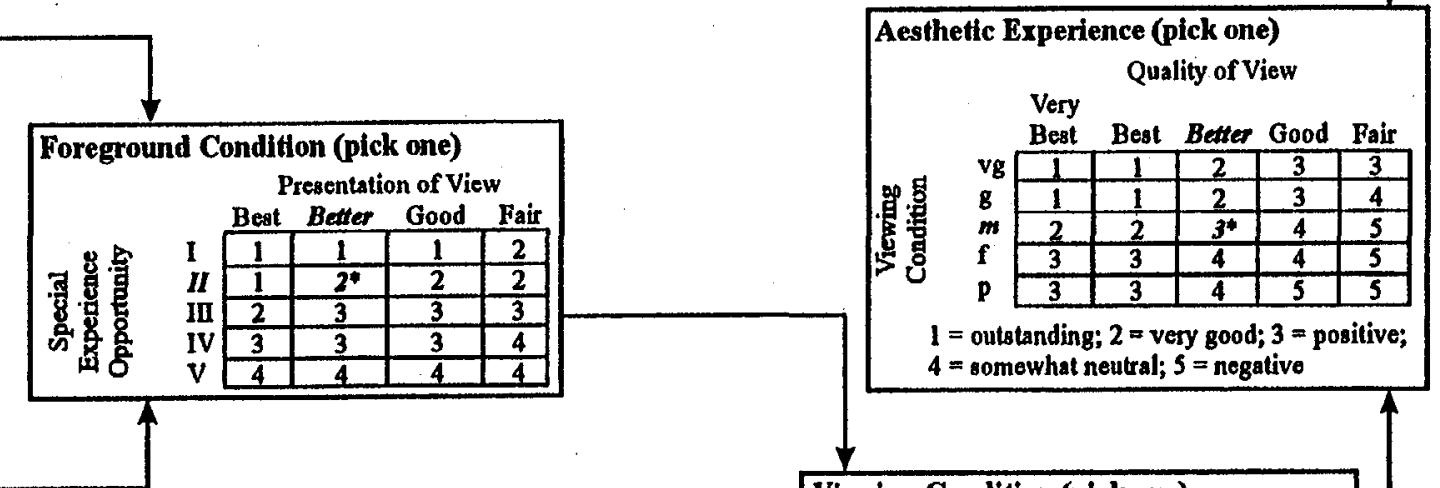

$1=$ outstanding: $2=$ very good; $3=$ positive; $4=80$ mewhat neutral; $5=$ negativo

Opportunity of View (pick one)

A. Horizontal view angle $>180^{\circ}$; vertical viow angle $>20^{\circ}$; pull

B. Horizontal view angle $90-180^{\circ}$; vertical view angle $15-20^{\circ}$;

C.* Horizontal view angle $45-90^{\circ}$; vertical angle $10-15^{\circ}$; pull over availablo; larger viewing angles with no pull over

D. Horizontal view angle $30-45^{\circ}$; no pull over available

B. Horizontal view angle $<30^{\circ}$; no pull over 


\section{Aesthetic Experience Worksheet}

Foothills Parkway Extension 8-B

*** Views From the Parkway Extension ***

\section{View 'Q', East end climb}

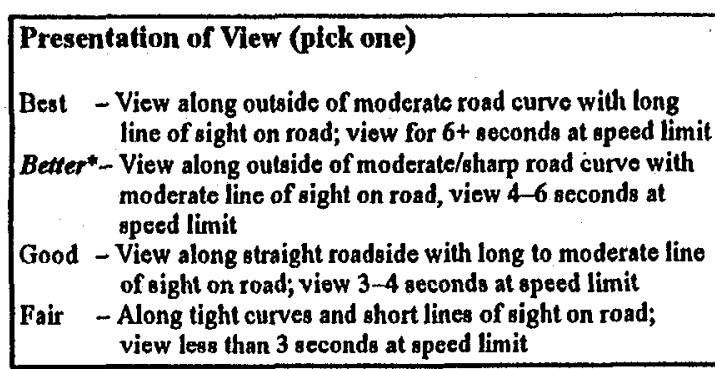

Special Experience Opportunity (pick one)

Fall color, spring blooms; water sounds; cool in summer, very special lighting effects

II. Morning or evening back/side lighting; mists; winter ice/frosts; deep woods; cool wet smelle

III. Wildlife viewing (birds, deer, etc.); special geology; cool woods smell; big trees; unusual vegetation; noticeable ecological processes

IV.* Mostly normal mountain vegetation; little unusual lighting effects or sensory experiences expected

V. Blinding sun; heavy fog; bad smells; bad traffic situation; noisy; strong winds; dead and dying vegetation; hot in summer; trash in view

Choices are marked with an asterisk.

\begin{tabular}{|c|c|}
\hline \multicolumn{2}{|c|}{ Quality of View (pick one) } \\
\hline \multicolumn{2}{|c|}{$\begin{array}{l}\text { Very Best - Water scenes with long viows of series of } \\
\text { receding mountain ridges and valleys }\end{array}$} \\
\hline Best & $\begin{array}{l}\text { - Long views of receding mountain ridges or } \\
\text { water scenes but not both; close views of } \\
\text { wooded mountain streams }\end{array}$ \\
\hline Better* & $\begin{array}{l}\text { - Long but partially blocked views of mountain } \\
\text { ridges or water scenes; more midground ridges } \\
\text { than long view }\end{array}$ \\
\hline & $\begin{array}{l}\text { - Midground views of opposing ridges; rustic } \\
\text { valleys, little to no long views }\end{array}$ \\
\hline Fair & $\begin{array}{l}\text { - Midground to foreground views of opposing } \\
\text { ridges, close views of roads, traffic; } \\
\text { development may be present }\end{array}$ \\
\hline
\end{tabular}

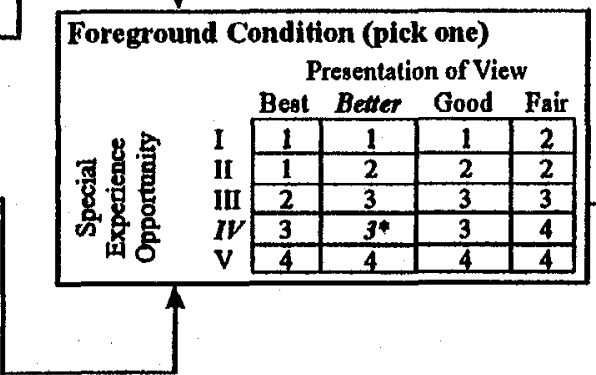

Opportunity of View (pick one)

A. Horizontal view angle $>180^{\circ}$; vertical view angle $>20^{\circ}$; pull over present

B. Horizontal view angle $90-180^{\circ}$; vertical view angle $15-20^{\circ}$; pull over present

C. * Horizontal view angle $45-90^{\circ}$; vertical anglo $10-15^{\circ}$; pull over available; larger viewing angles with no pull over vailable

D. Horizontal view angle $30-45^{\circ}$; no pull over available

E. Horizontal view angle $<30^{\circ}$; no pull over

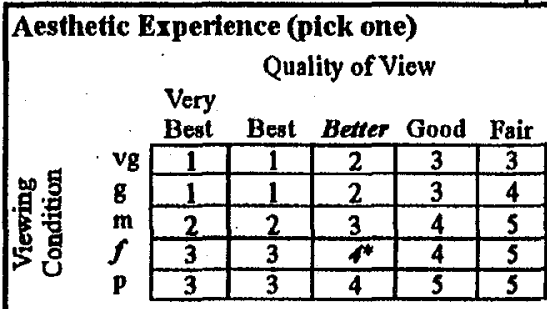

$1=$ oulstanding; $\mathbf{2}$ = very good; $\mathbf{3}=$ positive; $4=$ somewhat neutral; 5 - negative<smiles>[3H][3H]</smiles>

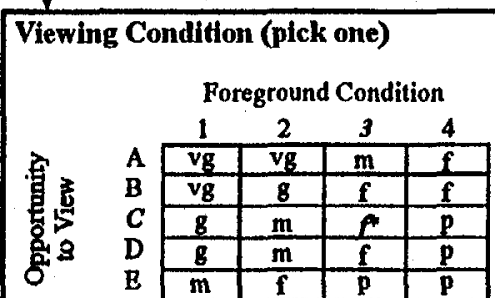

$\mathrm{vg}$ = very good; $g=$ good; $m=$ moderate; $p=$ poor 


\section{Aesthetic Experience Worksheet}

Foothills Parkway Extension 8-B *** Views From the Parkway Extension ***

\section{View '18', East bridge over 321 EW, 23-800}

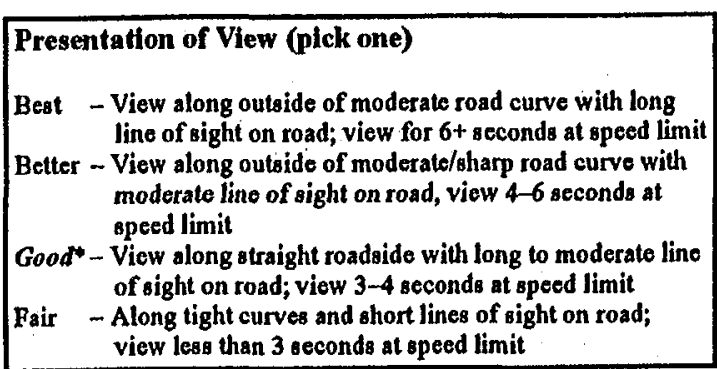

Special Experience Opportunity (pick one)

1. Fall color, spring blooms; water sounds; cool in summer; very special lighting effects

II.* Morning or evening back/side lighting; mists; winter

ice/frosts; deep woods; cool wet smells

III. Wildlife viewing (birds, deer, etc.); special geology; cool woods smell; big trees; unusual vegetation; noticeablo ecological processes

IV. Mostly normal mountain vegetation; little unuaual lighting effects or sensory experiences expected

Blinding sun; heavy fog: bad amelis; bad traffic situation; noisy; otrong winds; dead and dying vegetation; hot in summer; trash in view

Choices are marked with an asterisk.

\begin{tabular}{|c|c|}
\hline \multicolumn{2}{|c|}{ Quality of View (pick one) } \\
\hline \multicolumn{2}{|c|}{$\begin{array}{c}\text { Very Best - Water scenes with long viows of series of } \\
\text { receding mountain ridges and valleys }\end{array}$} \\
\hline Best & $\begin{array}{l}\text { - Long views of receding mountain ridges or } \\
\text { water scenes but not both; close views of } \\
\text { wooded mountain stroams }\end{array}$ \\
\hline Better* & $\begin{array}{l}\text { - Long but partially blocked viows of mountain } \\
\text { ridges or water scenes; more midground ridges } \\
\text { than long view }\end{array}$ \\
\hline & $\begin{array}{l}\text { - Midground views of opposing ridges; rustic } \\
\text { valloys, little to no long views }\end{array}$ \\
\hline Pair & $\begin{array}{l}\text { - Midground to foreground views of opposing } \\
\text { ridges, closo views of roads, traffic; } \\
\text { development may be present }\end{array}$ \\
\hline
\end{tabular}

\section{$\checkmark$}

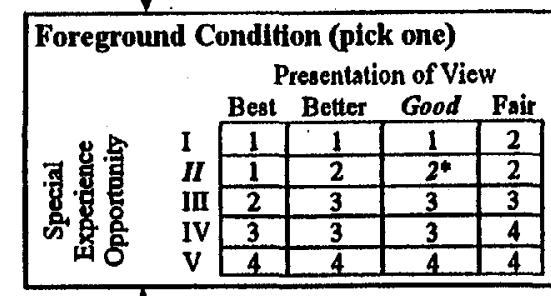

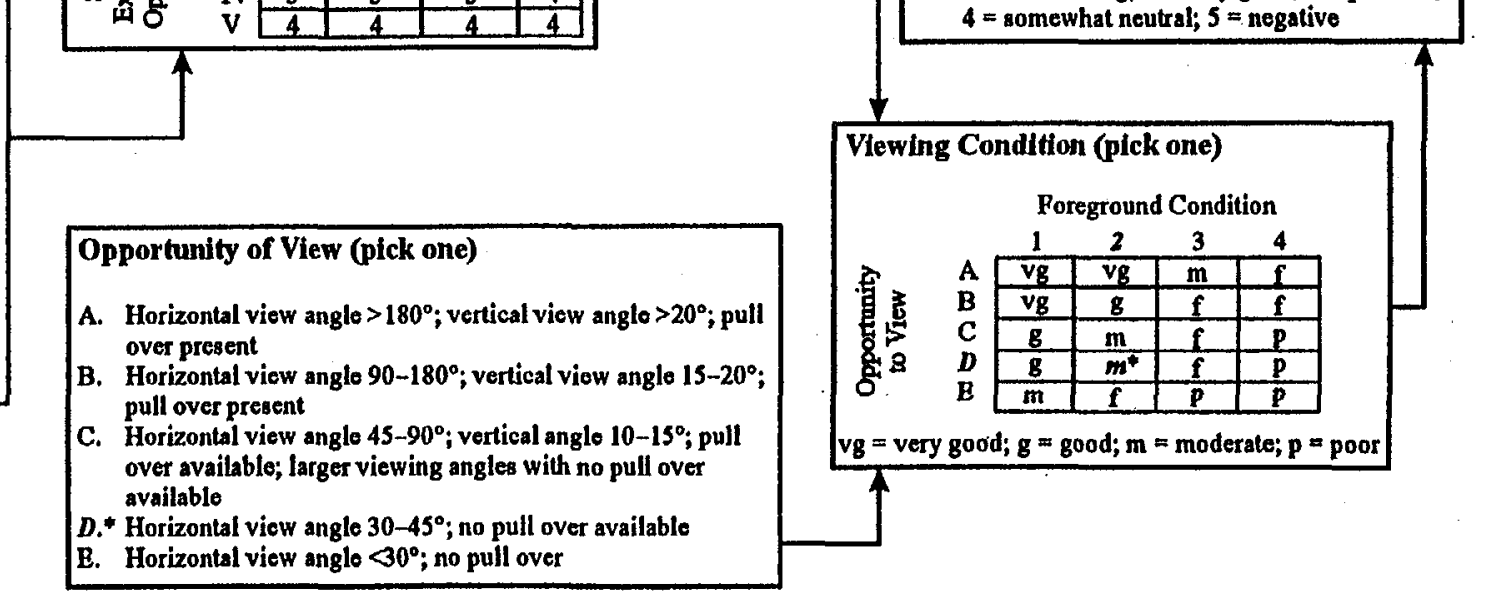




\section{Aesthetic Experience Worksheet Foothills Parkway Extension 8-B *** Views From the Parkway Extension *** \\ Walk-Up Overlook on Webb Min., km 8.600}

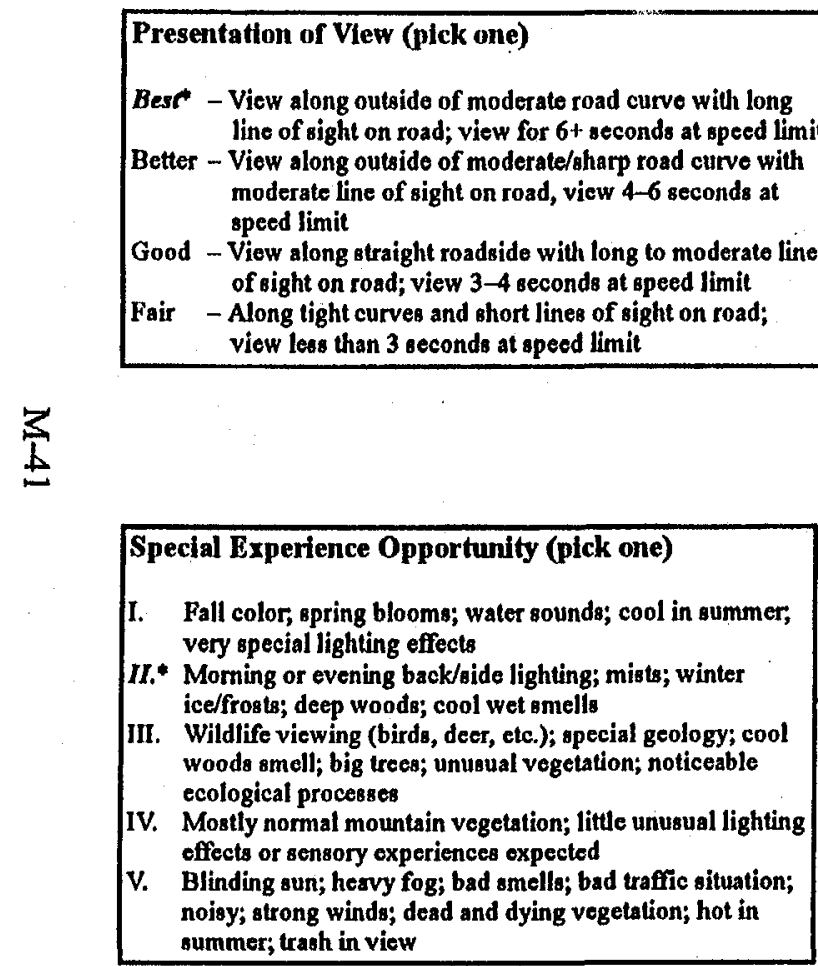

Choices are marked with an asterisk.

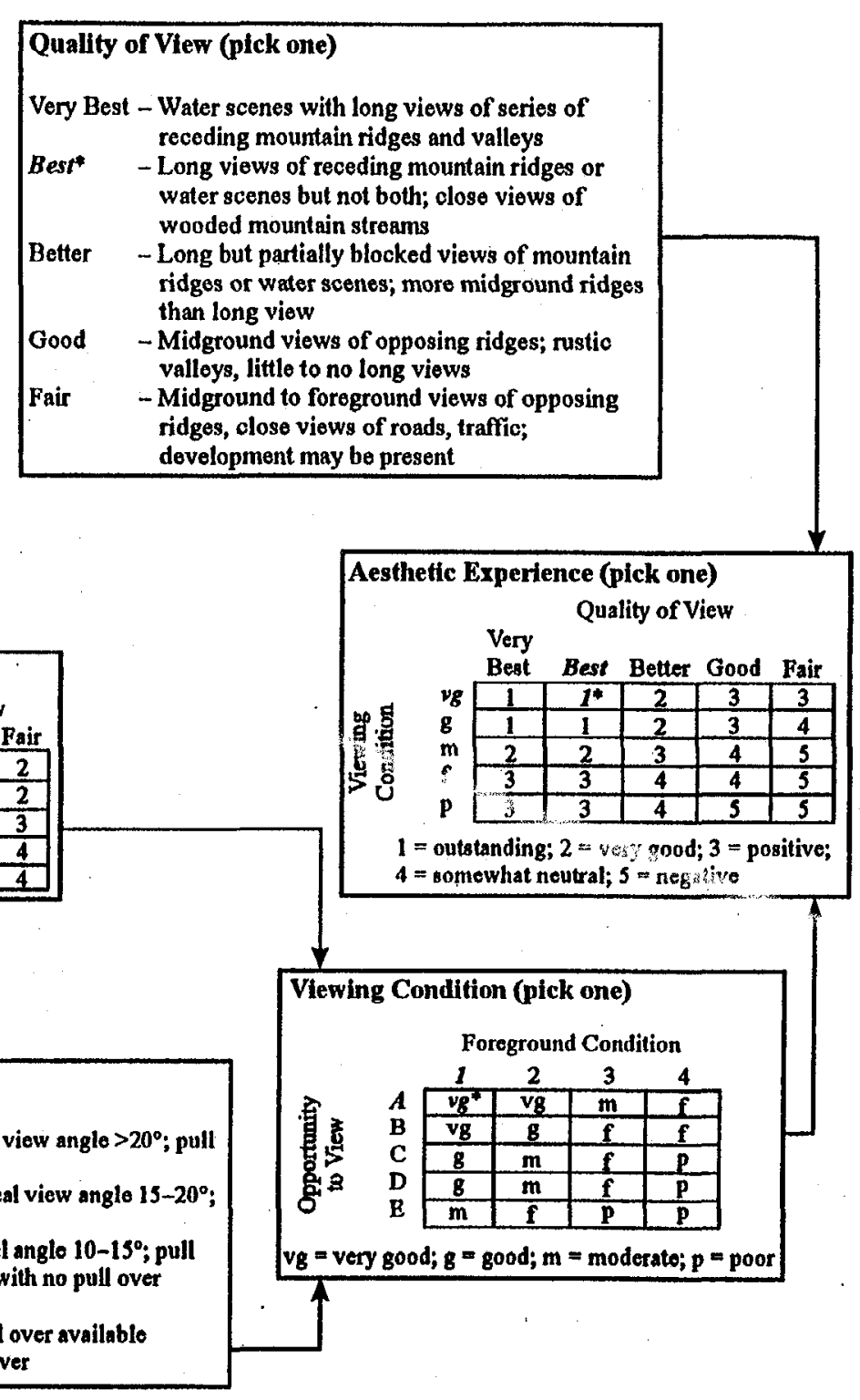

Opportunity of View (pick one)

$A *^{*}$ Horizontal view angle $>180^{\circ}$; vertical view angle $>20^{\circ}$; pult over present

B. Horizontal view angle $90-180^{\circ}$; vertical view angle $15-20^{\circ}$; pull over present

C. Horizontal view angle $45-90^{\circ}$; vertical anglo $10-15^{\circ}$; pull over availablo; larger viewing angles with no pull over available

D. Horizontal view angle $30-45^{\circ}$; no pull over availablo

E. Horizontal view anglo $<0^{\circ}$; no pull over

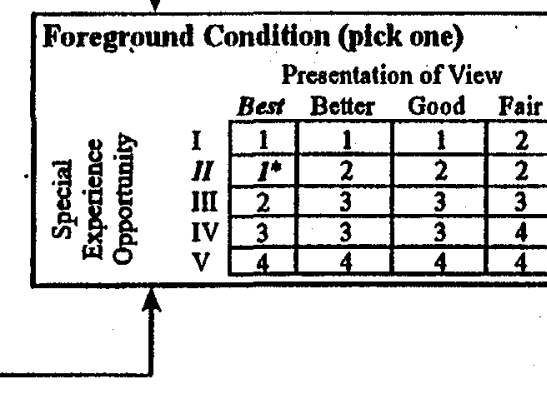

$1=$ outstanding; 2 =t yery good; 3 = positive; $=$ somewhat neutral; $5=$ negastive

$g=$ very good; $g=$ good; $m=$ moderato; $p=$ poor 